Methods for Addressing Uncertainty and Variability to Characterize Potential Health Risk from TrichloroethyleneContaminated Ground Water at Beale Air Force Base in California: Integration of Uncertainty and Variability in Pharmacokinetics and Dose-Response

U.S. Department of Energy

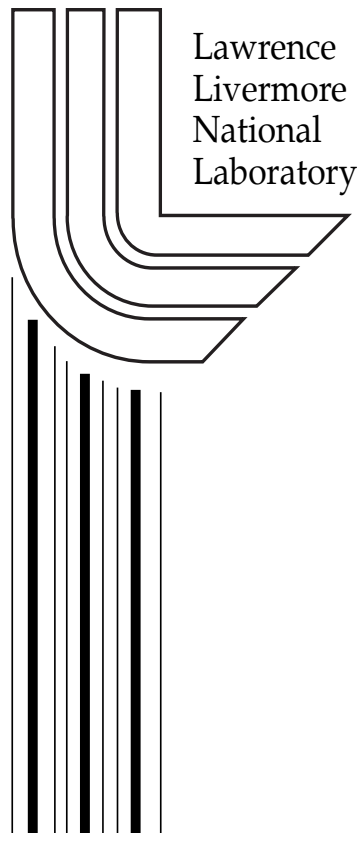

K. T. Bogen

May 24, 2001 


\section{DISCLAIMER}

This document was prepared as an account of work sponsored by an agency of the United States Government. Neither the United States Government nor the University of California nor any of their employees, makes any warranty, express or implied, or assumes any legal liability or responsibility for the accuracy, completeness, or usefulness of any information, apparatus, product, or process disclosed, or represents that its use would not infringe privately owned rights. Reference herein to any specific commercial product, process, or service by trade name, trademark, manufacturer, or otherwise, does not necessarily constitute or imply its endorsement, recommendation, or favoring by the United States Government or the University of California. The views and opinions of authors expressed herein do not necessarily state or reflect those of the United States Government or the University of California, and shall not be used for advertising or product endorsement purposes.

This is a preprint of a paper intended for publication in a journal or proceedings. Since changes may be made before publication, this preprint is made available with the understanding that it will not be cited or reproduced without the permission of the author.

This work was performed under the auspices of the United States Department of Energy by the University of California, Lawrence Livermore National Laboratory under contract No. W-7405-Eng-48.

This report has been reproduced directly from the best available copy.

Available electronically at http://www.doc.gov/bridge

Available for a processing fee to U.S. Department of Energy

And its contractors in paper from

U.S. Department of Energy

Office of Scientific and Technical Information

P.O. Box 62

Oak Ridge, TN 37831-0062

Telephone: (865) 576-8401

Facsimile: (865) 576-5728

E-mail: reports@adonis.osti.gov

Available for the sale to the public from

U.S. Department of Commerce

National Technical Information Service

5285 Port Royal Road

Springfield, VA 22161

Telephone: (800) 553-6847

Facsimile: (703) 605-6900

E-mail: orders@ntis.fedworld.gov

Online ordering: http://www.ntis.gov/ordering.htm

Or

Lawrence Livermore National Laboratory

Technical Information Department's Digital Library

http://www.llnl.gov/tid/Library.html 


\title{
Methods for Addressing Uncertainty and Variability to Characterize Potential Health Risk from Trichloroethylene-Contaminated Ground Water at Beale Air Force Base in California:
}

\section{Integration of Uncertainty and Variability in Pharmacokinetics and Dose-Response}

\author{
K.T. Bogen \\ Lawrence Livermore National Laboratory, \\ University of California, Livermore, $\mathrm{CA}^{*}$
}

May 24, 2001

*Address correspondence to: Dr. K.T. Bogen, Health and Ecological Assessment Div. L-396, Lawrence Livermore National Laboratory, Livermore, CA 94550-9234, USA, Tel: (925) 422-0902, Fax: (925) 424-3255, NET: bogen@LLNL.gov. 


\section{ACKNOWLEDGMENTS}

I am very grateful to the Institute for Environmental, Safety, and Occupational Risk Analysis (IERA), Environmental Science Branch (RSRE), of the U.S. Air Force (USAF) $311^{\text {th }}$ Human System Wing, located at Brooks Air Force Base in Texas, for providing technical guidance and support for this work. I also thank Dr. John Christopher and Dr. Brian Davis of the State of California Environmental Protection Agency (CalEPA), Department of Toxic Substances Control (DTSC), for technical advice and comments during the development of this report. I thank Dr. Jeffrey Daniels at LLNL for his

previous collaboration with me on Phase 1 of this study, results from which are cited and used in the present report. Finally, I am grateful to the Chief for Environmental Restoration at Beale Air Force Base in California, and the Headquarters of the Air Combat Command at Langley Air Force Base in Virginia, for providing and permitting the use of data from Beale Air Force Base as a groundwater contamination scenario serving to illustrate methods developed and demonstrated in this study. This work was performed under the auspices of the U.S. Department of Energy by the University of California at Lawrence Livermore National Laboratory under contract W-7405-Eng-48. 


\section{TABLE OF CONTENTS}

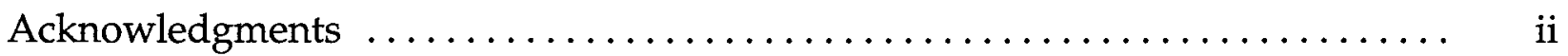

Table of Contents $\ldots \ldots \ldots \ldots \ldots \ldots \ldots \ldots \ldots \ldots \ldots \ldots \ldots \ldots \ldots \ldots \ldots$, iii

List of Tables and Figures $\ldots \ldots \ldots \ldots \ldots \ldots \ldots \ldots \ldots \ldots \ldots \ldots \ldots \ldots \ldots \ldots \ldots, \mathrm{v}$

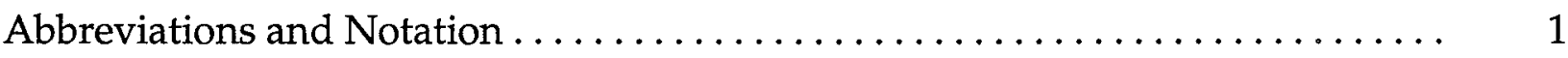

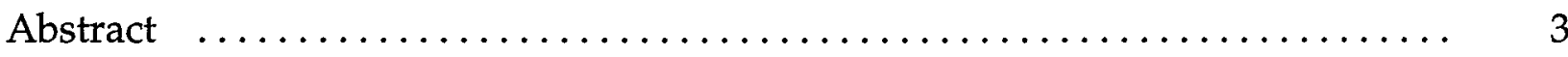

1. Introduction $\ldots \ldots \ldots \ldots \ldots \ldots \ldots \ldots \ldots \ldots \ldots \ldots \ldots \ldots \ldots \ldots \ldots, 4$

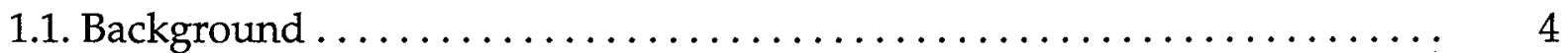

1.2. Importance of Quantitative Analysis of Joint Uncertainty and Variability 6

1.3. Uncertainty in Mechanism(s) of Toxic Action ................. 7

1.4. Technical Issues Posed by Quantitative Analysis of Joint Uncertainty and Variability in Dose-Response for TCE-Induced Risk ........... 10

1.5. Study Objectives . . . . . . . . . . . . . . . . . . . . . . . . . . . 14

2. Methods ........................................... 14

2.1. Systematic Probabilistic Framework $\ldots \ldots \ldots \ldots \ldots \ldots \ldots \ldots \ldots . \quad 15$

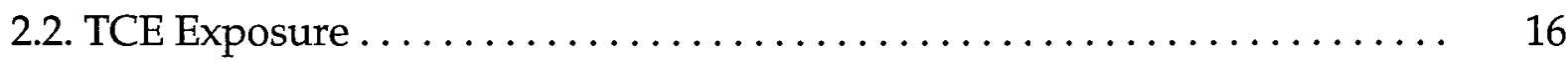

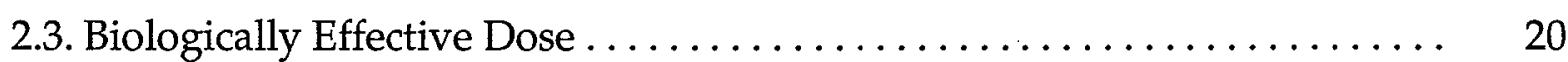

2.3.1. Uncertainty in mechanism of toxic action ............... 21

2.3.2. PBPK modeling approach $\ldots \ldots \ldots \ldots \ldots \ldots \ldots \ldots \ldots \ldots, 22$

2.3.3. Effective genotoxic dose $\ldots \ldots \ldots \ldots \ldots \ldots \ldots \ldots \ldots \ldots .23$

2.3.4. Effective cytotoxic dose $\ldots \ldots \ldots \ldots \ldots \ldots \ldots \ldots \ldots \ldots \ldots .25$

2.4. TCE Dose-Response .............................. 29

2.4.1. Dose-response assuming genotoxic mechanism(s) . ........... 29

2.4.2. Dose-response assuming cytotoxic mechanism(s) ........... 32

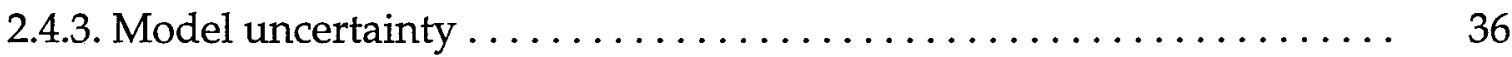

2.5. Risk Characterization ............................... 37

2.6. Data Analysis and Computation ........................ 38

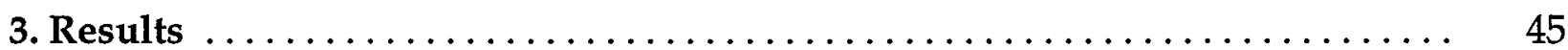

3.1. Biologically Effective Dose $\ldots \ldots \ldots \ldots \ldots \ldots \ldots \ldots \ldots \ldots, 45$

3.2. Dose-Response ................................. 48

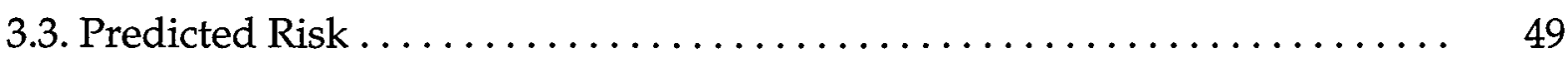




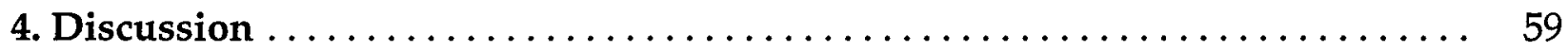

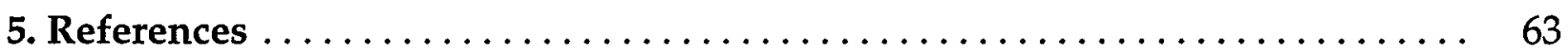

Appendix 1. Method of Moments for Lognormal Variates .............. 70

Appendix 2. The Exact Distribution of a Linear Function of Correlated

$t$-Distributed Variates . . . . . . . . . . . . . . . . . . .

Appendix 3. Documentation of Mathematica $4.0^{\circledast}$ and RiskQ Calculations ....... . 75
A. Concentration
$\mathrm{A} 1-\mathrm{A} 2$
B. Intakes ...
$\mathrm{B} 1-\mathrm{B} 12$
C. Fraction of Lifetime at One Local Residence
$\mathrm{C} 1-\mathrm{C} 8$
D. Effective (TCE) Genotoxic Dose
D1 - D10
E. Effective (TCA) Cytotoxic Dose
E1 - E14
F. Effective Dose Correlations
F1 - F8
G. Potency .
G1 - G18
H. TCE Risk .
$\mathrm{H} 1$ - $\mathrm{H} 22$
I. Functions Used
I1 - I7 


\section{LIST OF TABLES AND FIGURES}

\section{Tables}

Table 1. Bioassay data sets used to estimate cancer potency of TCE as a genotoxic/linear liver or kidney carcinogen $\ldots \ldots \ldots \ldots \ldots \ldots .$.

Table 2. Constants and variates used as input for unified TCE risk assessment

Table 3. Rank correlations among uncertainty-expectations of normalized

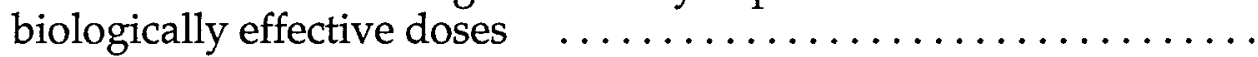

Table 4. Summary of estimated risk posed by TCE in ground water at Site LF-13

Table 5. Population risk associated with multipathway exposures to ground water containing low-level concentrations of trichloroethylene (TCE)

\section{Figures}

Figure 1. Systematic probabilistic framework to risk assessment for TCE ....

Figure 2. Approximation of the ratio $\left(f_{\text {deq }} / V_{t, \mathrm{P}}\right) \ldots \ldots \ldots \ldots \ldots \ldots \ldots \ldots .28$

Figure 3. Limiting metabolized fractions $V_{\mathrm{fm}, \mathrm{P}}$ of low-level TCE absorbed via different routes

Figure 4. Expectations with respect to uncertainty vs. variability in normalized effective genotoxic and cytotoxic dose $\ldots \ldots \ldots \ldots \ldots \ldots \ldots \ldots$

Figure 5. Estimation error (uncertainty) in cancer potency based on

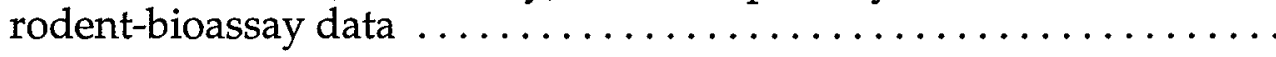

Figure 6. Fit and extrapolation of lognormal model to mouse cytotoxicity

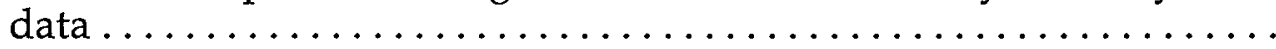

Figure 7. Risk of cytotoxic response $\ldots \ldots \ldots \ldots \ldots \ldots \ldots \ldots \ldots \ldots$

Figure 8. Uncertainty in population-average risk, $\bar{R}$, and interindividual variability in expected risk, $\langle R\rangle \ldots \ldots \ldots \ldots \ldots \ldots \ldots \ldots \ldots . .$.

Figure 9. Comparison of $\bar{R}$ vs. $\langle R\rangle$ over different ranges of predicted risk...

Figure 10. Comparison of estimators $\left(R_{u, v}\right)$ of joint uncertainty and variability

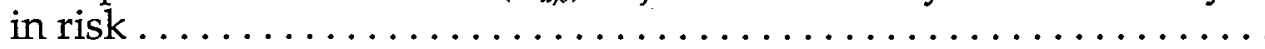




\section{ABBREVIATIONS and NOTATION}

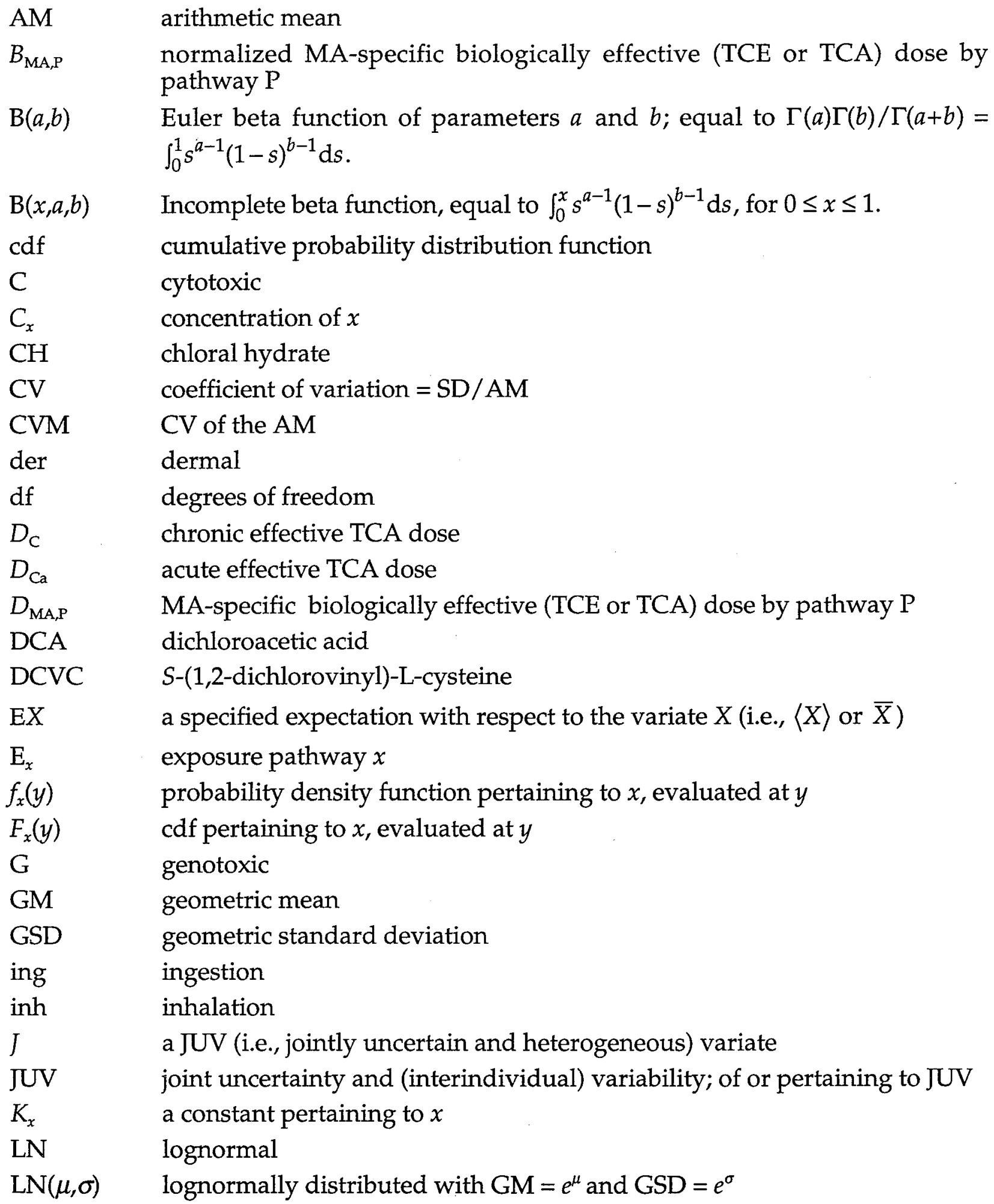




\begin{tabular}{|c|c|}
\hline LTWA & lifetime time-weighted average \\
\hline MA & mechanistic assumption, i.e., assumed mode/mechanism of action \\
\hline $\mathrm{N}(\mu, \sigma)$ & normally distributed with $\mathrm{AM}=\mu$ and $\mathrm{SD}=\sigma$ \\
\hline NRC & National Research Council \\
\hline NTP & National Toxicology Program \\
\hline$P$ & pathway or route of TCE exposure (intake) \\
\hline$P$ or $p$ & probability \\
\hline$p_{\text {adj }}$ & adjusted probability \\
\hline PBPK & physiologically based pharmacokinetic \\
\hline$r$ & product-moment correlation \\
\hline$R$ & $\begin{array}{l}\text { increased individual lifetime probability of incurring a toxic (cancer } \\
\text { and/or noncancer) endpoint due to TCE exposure }\end{array}$ \\
\hline SD & standard deviation \\
\hline SDM & SD of the AM \\
\hline$t$ & time, or reference to Student's $t$-distribution \\
\hline TCA & trichloroacetic acid \\
\hline TCE & trichloroethylene \\
\hline USEPA & U.S. Environmental Protection Agency \\
\hline$U_{x}$ & an uncertain variate pertaining to $x$ \\
\hline$V_{x}$ & $\begin{array}{l}\text { a "heterogeneous" variate pertaining to } x \text {; i.e., different variate values } \\
\text { pertain to different individuals at risk }\end{array}$ \\
\hline VOC & volatile organic compound \\
\hline $\mathbf{x}$ & vector of uncertain and/or heterogeneous variates, $\left\{X_{1}, X_{2}, \ldots\right\}$ \\
\hline$\langle X\rangle$ & expectation with respect to uncertainty pertaining to the variate $X$ \\
\hline $\bar{X}$ & $\begin{array}{l}\text { expectation with respect to interindividual variability pertaining to } \\
\text { the variate } X\end{array}$ \\
\hline $\mathrm{X}_{\mathrm{MA}, \mathrm{P}}$ & MA-specific TCE exposure (intake) by pathway $\mathrm{P}$ \\
\hline$\beta(x, a, b)$ & $\begin{array}{l}\text { Incomplete beta function ratio, or regularized incomplete beta function, } \\
\text { equal to } \mathrm{B}(x, a, b) / \mathrm{B}(a, b) \text {, for } 0 \leq x \leq 1 \text {. }\end{array}$ \\
\hline$\Phi(z)$ & cdf of a standard normal (Gaussian) variate $Z,=\operatorname{Prob}(Z \leq z)$ \\
\hline$\rho_{\mathrm{r}}(x, y)$ & rank correlation coefficient between variates $x$ and $y$ \\
\hline
\end{tabular}




\begin{abstract}
Traditional estimates of health risk are typically inflated, particularly if cancer is the dominant endpoint and there is fundamental uncertainty as to mechanism(s) of action. Risk is more realistically characterized if it accounts for joint uncertainty and interindividual variability within a systematic probabilistic framework to integrate the joint effects on risk of distributed parameters of all (linear as well as nonlinear) riskextrapolation models involved. Such a framework was used to characterize risks to potential future residents posed by trichloroethylene (TCE) in ground water at an inactive landfill site on Beale Air Force Base in California. Variability and uncertainty were addressed in exposure-route-specific estimates of applied dose, in pharmacokinetically based estimates of route-specific metabolized fractions of absorbed TCE, and in corresponding biologically effective doses estimated under a genotoxic/linear $\left(\mathrm{MA}_{\mathrm{G}}\right)$ vs. a cytotoxic/nonlinear $\left(\mathrm{MA}_{\mathrm{C}}\right)$ mechanistic assumption for TCE-induced cancer. Increased risk conditional on effective dose was estimated under $\mathrm{MA}_{\mathrm{G}}$ based on seven rodent-bioassay data sets, and under $M \mathrm{~A}_{C}$ based on mouse hepatotoxicity data. Mean and upper-bound estimates of combined risk calculated by the unified approach were $<10^{-6}$ and $<10^{-4}$, respectively, while corresponding estimates based on traditional deterministic methods were $>10^{-5}$ and $>10^{-4}$, respectively. It was estimated that no TCE-related harm is likely to occur due to any plausible residential exposure scenario involving the site. The systematic probabilistic framework illustrated is particularly suited to characterizing risks that involve uncertain and/or diverse mechanisms of action.
\end{abstract}




\section{INTRODUCTION}

This report describes methods and results pertaining to Phase 2 of a study involving quantitative consideration of joint uncertainty and interindividual variability in risk to hypothetical future residents posed by trichloroethylene (TCE) in ground water at the inactive landfill Site LF-13 on Beale Air Force Base in California. The background of this study is discussed below, followed by summaries of: the rationale for this study's focus on quantitative analysis of joint uncertainty and variability; technical hurdles posed by undertaking such an analysis in a way that explicitly addresses carcinogenic dose-response of TCE in view of fundamental uncertainty concerning its carcinogenic mode of action; and study goals of Phase 2 of the analysis undertaken of risk posed by TCE at Site LF-13. Specific methods used to address the latter goals are presented in Section 2 of this report. Results obtained by applying these methods are presented in Section 3, followed in Section 4 by a discussion of the results obtained. References cited in this report are listed in Section 5. Appendix 1 supplies mathematical details concerning the "method of moments" used throughout in this report to make assumptions about lognormal variates. Appendix 2 supplies a proof concerning the distribution of a linear function of correlated $t$-distributed variates. Finally, Appendix 3 documents all calculations performed for this study.

The general background of the present study and its Phase- 1 counterpart is provided in Section 1.1 below, followed by: a summary of the rationale for the emphasis in this report placed on quantitative analysis of joint uncertainty and variability (Section 1.2), a discussion of the present fundamental uncertainty pertaining to mechanism(s) of action for TCE-induced cancer (Section 1.3), issues involving quantitative analysis of joint uncertainty and variability in dose-response for TCEinduced cancer (Section 1.4), and the specific goals of the present report (Section 1.5).

\subsection{Background}

Traditional point estimates of risk are calculated deterministically using worst-case assumptions for some or all input parameters, in a way that does not quantitatively account for uncertainty and interindividual variability pertaining to these parameters. Traditional point-estimates of risk are thus typically inflated and health-conservative, particularly if the cancer is the dominant endpoint and there is fundamental uncertainty 
as to mechanism(s) of action. Risk is more realistically characterized if it accounts for joint uncertainty and interindividual variability within a systematic probabilistic framework to integrate the joint effects on risk of distributed parameters in all (linear as well as nonlinear) risk-extrapolation models for all (cancer as well as noncancer) endpoints involved. Because no such systematic probabilistic framework existed, one was developed for the present case study involving the inactive Landfill Site LF-13 on Beale Air Force Base in California, where groundwater contaminated with trichloroethylene (TCE) has moved beyond the site boundary. Soil-vapor extraction and air-stripping treatment of groundwater have been undertaken to reduce concentrations of TCE and other volatile organic compounds in ground water beneath Site LF-13 (URSGWC, 1998). Site LF-13 is located in a currently rural area of the Sacramento Valley of California, where groundwater wells are the principle source of domestic water supplies. The present analysis was undertaken to provide a realistic characterization of hypothetical TCE-related risks associated with potential future domestic/residential uses of groundwater from beneath Site LF-13, in view of the possibility that residential populations may eventually occupy lands adjacent to the site.

This study was conducted in two phases. Phase 1 focused on the impact of joint uncertainty and interindividual variability (JUV) on estimates of combined TCE intakes via different exposure pathways (Daniels et al., 1999, 2000). Uncertainty here refers to an absence of measurement data or incomplete knowledge; interindividual variability (or "variability") here refers to true differences or heterogeneity in an empirical, riskrelated characteristic (e.g., physiological differences) among individuals in a population (Bogen and Spear, 1987; NRC, 1994). Although results of the Phase 1 analysis were presented as a characterization of risk rather than exposure, risk was estimated in that analysis simply as the product of estimated combined exposures (in $\mathrm{mg} \mathrm{kg}^{-1} \mathrm{~d}^{-1}$ ) and respective factors representing carcinogenic potency (in $\mathrm{kg} \mathrm{d} \mathrm{mg}^{-1}$ ), each of which factor was represented by a single point estimate. Thus, JUV in risk characterized in Phase 1 reflected only JUV in estimated exposure, and in no way addressed JUV associated with TCE pharmacokinetics, dose-response, alternative mechanisms of toxic action, or multiple toxic endpoints. The TCE concentration in Phase 1 was estimated based on groundwater-monitoring data for a well on Site LF-13 near the possible location of a future groundwater extraction and distribution system (Purrier, 1997). After 
considering concentration uncertainty and JUV in potential multi-route exposures to TCE from Site LF-13 ground water, corresponding JUV in risk was characterized and compared to corresponding risk estimators that were calculated using traditional deterministic methods (Daniels et al., 2000).

Phase 2 of the study described above is the subject of the present report. Phase 2 involved the development of new methods allowing additional information to be integrated into a Phase-1-type TCE risk assessment for Site LF-13. This additional information involves JUV in predicted risk conditional on route-specific TCE exposures. As further explained below, this was accomplished by combining exposure distributions and methods presented in the Phase-1 study with TCE-related pharmacokinetic and dose-response methods and information developed in the present study, to provide an improved characterization of TCE-related risk associated with Site LF-13 at Beale AFB.

\subsection{Importance of Quantitative Analysis of Joint Uncertainty and Variability}

This study focuses on integrating information on joint uncertainty and interindividual variability (JUV) to obtain more meaningful and more realistic estimates of exposure and risk. In the report, Science and Judgment in Risk Assessment, the National Research Council (NRC) emphasized the importance of distinguishing clearly between uncertainty (i.e., lack of knowledge) and interindividual "variability" (i.e., heterogeneity or differences pertaining to people at risk) in risk assessment (NRC, 1994). Uncertainty in characterized risk reflects the extent to which a risk estimate is likely to be erroneous, due to gaps in data and/or theory that imply statistical and/or model-specification error. Interindividual variability in characterized risk reflects the extent to which a risk is unequally imposed on members of the population at risk. While uncertainty reduces the confidence or reliability that can be placed in a risk estimate, variability can be viewed as a measure of perceived unfairness or inequity represented by the distribution of imposed risks. Because reliability and equity issues are clearly related to perceived and/or statutorily defined risk acceptability criteria, both these dimensions may be relevant to risk-management policy decisions.

Quantitative characterization of joint uncertainty and variability (JUV) in risk is a way to address risk-related uncertainty and variability concisely and explicitly to 
facilitate risk management decisions. When JUV is addressed quantitatively in the input distributions used to characterize the inputs (e.g., on ambient concentration, uptake, and dose-response) of a risk assessment, the distinction between uncertainty and variability ought to be maintained rigorously throughout the analytic process so that uncertainty and variability can be reflected distinctly in the calculated risk. This recommendation was expressed by the NRC (1994, p. 242) as follows:

"A distinction between uncertainty (i.e., degree of potential error) and inter-individual variability (i.e., population heterogeneity) is generally required if the resulting quantitative risk characterization is to be optimally useful for regulatory purposes, particularly insofar as risk characterizations are treated quantitatively. The distinction between uncertainty and individual variability ought to be maintained rigorously at the level of separate risk-assessment components (e.g., ambient concentration, uptake, and potency) as well as at the level of an integrated risk characterization."

If no distinction is made between uncertainty-related and heterogeneity-related distributions associated with inputs to a given risk calculation, then the resulting distribution necessarily reflects risk to an individual selected at random from the exposed population (Bogen and Spear, 1987). By definition, this resulting distribution cannot be used for any regulatory decision intending to address equity issues by focusing on risk borne by relatively more sensitive and/or relatively more highly exposed members of the population at risk. Another advantage of distinguishing between uncertainty and variability is that it permits one to estimate the uncertainty in the risk to the individual who is "average" with respect to all characteristics that are heterogeneous among individuals at risk. Only the latter quantity can be used to estimate corresponding uncertainty in predicted population risk (i.e., uncertainty in the predicted number of cases), and thus, in particular, to estimate the likelihood of zero cases (i.e., the likelihood that remediation of the exposure scenario considered will have no positive impact whatsoever on public health) (Bogen and Spear, 1987).

\subsection{Uncertainty in Mechanism(s) of Toxic Action}

Liver is clearly a target tissue for TCE-induced cancer based on lifetime bioassay data on chronically exposed mice; relatively large acute, subchronic, or chronic TCE exposures are hepatotoxic in multiple species; and hepatocellular toxicity in mice is the most sensitive TCE-induced noncancer (but possibly cancer-related) endpoint (Bogen 
and Gold, 1997; Bogen et al., 1988; USEPA, 1985). Limited epidemiological data also support liver cancer as a TCE-induced endpoint in occupationally exposed humans (Wartenberg, 2000). DNA-binding and weak mutagenicity associated with TCE metabolites after TCE administration indicates that genotoxicity may be responsible for some or all TCE-induced cancer (Bogen and Gold, 1997; Fahrig et al., 1995). Two TCE metabolites in particular, trichloroacetic acid (TCA) and dichloroacetic acid (DCA), both induce and promote liver tumors in a mouse strain (B6C3F1) which is positive for TCEinduced liver cancer, whereas liver tumors did not appear in rats exposed to either TCE by gavage or to TCA via drinking water (Bogen and Gold, 1997; Bull et al., 1990; DeAngelo et al., 1997; DeAngelo et al., 1991; Herren-Freund et al., 1987; Pereira, 1996; Pereira and Phelps, 1996). DCA in particular was found recently to be weakly mutagenic in mouse lymphoma cells with a mutagenic potency similar to the classic mutagen ethyl methanesulfonate, whereas only very weak mutagenic activity was detected using either the major reactive TCE metabolite, chloral hydrate $(\mathrm{CH})$, or its breakdown product TCA (Harrington-Brock et al., 1998; Moore and Harrington-Brock, 2000). Initial studies found DCA to be more reactive and toxic than TCE, and thus more likely to account for observed TCE-induced cancer in bioassay mice (Larson and Bull, 1992a-b; Templin et al., 1993). However, more recent studies that controlled for ex vivo formation of DCA during sample preparation indicate that very little, if any, DCA was actually produced in TCE-exposed B6C3F1 mice, imply the same for humans as well (Lash et al., 2000a), and are consistent with the hypothesis that DCA is unlikely to explain TCE-induced mouse tumors (Andersen et al., 1998; Merdink et al., 1998) or to be relevant in extrapolating TCE-induced cancer risk for humans (Bull, 2000).

The generally weak mutagenicity of TCE and its metabolites (Moore and Harrington-Brock, 2000), as well as correlations between hepatotoxic indicators induced by reactive TCE metabolites and precursors to TCE-induced liver tumorigenesis (Bogen and Gold, 1997), provide substantial (although not definitive) support a cytotoxic mechanism of TCE-induced carcinogenic action. Hepatotoxic lipid peroxidation was found to be induced by TCA in mice and rats, but mice were found to be more sensitive than rats (Larson and Bull, 1992a). This differential sensitivity to a TCA-induced cytotoxic endpoint is consistent with a cytotoxicity-based explanation of TCE-induced liver tumors in mice but not rats. A more recent study of lipid peroxidation induced in 
B6C3F1 mouse liver concluded that the amount of such peroxidation induced by "TCA equaled that induced by $\mathrm{CH}$, whereas that from [trichloroethanol, another major, but less toxic and reactive, TCE metabolite] was 3- to 4-fold lower, suggesting that metabolism of $\mathrm{CH}$ to TCA may be the predominant pathway leading to lipid peroxidation" (Ni et al., 1996). Lipoperoxidation-induced oxidative stress may explain or correlate with the induction of hepatocellular replicative DNA synthesis and hepatocellular proliferation that has been observed in TCA-exposed B6C3F1 mice (Dees and Travis, 1994). Increased cell proliferation, in turn, either alone or in combination with genotoxic conditions, has long been considered sufficient to explain increased rates of cancer in view of biologically based mechanistic cell-kinetic multistage cancer theory, as well as based on experimental, epidemiological and clinical observations (Ames and Gold, 1990a; Ames and Gold, 1990b; Ames et al., 1993,1995; Armitage and Doll, 1957; Bogen, 1989; Cohen and Ellwein, 1990,1991; Moolgavkar, 1983; Moolgavkar and Knudson, 1981; Moolgavkar et al., 1988).

Statistical considerations support rejecting lung as a significant target site for TCEinduced cancer in rodents (Bogen and Gold, 1997). Epidemiological evidence of TCEinduced kidney cancer in humans has been characterized variously as "limited", "suggestive", and "neither consistent nor convincing," but additional recent data appear to indicate that kidney cancer risk is elevated among metabolically susceptible workers with very large TCE exposures (Lash et al., 2000b). While National Toxicology Program (NTP) bioassay data also indicate that kidney cancer is induced experimentally by chronic TCE exposure is the rat (but not mouse), these data were all judged to be "inadequate" after NTP review, with mild to severe renal toxicity observed at every non-control dose level in every species/sex combination in the bioassays (Bogen and Gold, 1997; NTP, 1988,1990). The NTP rats studies and limited/suggestive epidemiological data comprise the only evidence that TCE-induced renal tumors are plausibly relevant to humans (Bogen and Gold, 1997; Bogen et al., 1988; USEPA, 1985; Lash et al., 2000b). The rat tumor data are consistent with a cytotoxic mechanism of action for renal carcinogenesis, although mutagenicity of renal TCE-metabolites such as S-(1,2-dichlorovinyl)-L-cysteine (DCVC) indicates that genotoxicity may also play a role (Bogen and Gold, 1997; Fahrig et al., 1995; Lash et al., 2000a-b). Interestingly, while subchronically administered TCA and acutely administered DCVC were both found to 
be nephrotoxic and to induce cell proliferation in rat kidney tubules, the DCVDinduced response in mice (in which TCE-induced kidney tumors have not been observed) was much more pronounced than in rats (in which TCE-induced kidney tumors have been observed) (Acharya et al., 1997; Eyre et al., 1995). Consequently, the same uncertainties regarding the mechanism of TCE-induced hepatocarcinogenicity apply to the mechanism of possible TCE-induced renal tumors.

The U.S. Environmental Protection Agency (USEPA) has not explicitly endorsed the quantitative combination of "model" uncertainty with other types of uncertainty in cancer risk assessments for compounds like TCE (USEPA, 1996). This USEPA position is consistent with a recent NRC recommendation against this type of quantitative treatment as opposed to narrative/qualitative comparisons of model-specific analyses (NRC, 1994). However, the restriction of "model" uncertainty to be characterized only nonquantitatively needlessly reduces the clarity of risk analysis, because there is no logical merit to the distinction between "model" and "parameter" uncertainty. As the NRC report itself pointed out, the former is logically equivalent to the latter when incorporated into a suitably general model that specifies, through values assigned to one or more uncertain parameters, any particular but uncertain model characteristics (i.e., substructures) of concern (NRC, 1994; p. 187).

\subsection{Technical Issues Posed by Quantitative Analysis of Joint Uncertainty and Variability in Dose-Response for TCE-Induced Risk}

In view of the issues discussed above, there were several technical issues that had to be addressed in this study due to its primary focus on quantitative analysis of JUV in dose-response for TCE-induced risk. These issues concern the lack of coordinated methods that consistently and simultaneously address:

(i) multiple toxic (in this case, cancer and noncancer) endpoints with potentially disparate dose-response relations,

(ii) multiple plausible mechanisms of carcinogenic action,

(iii) efficient treatment of pharmacokinetic relations, and

(iv) integrated, quantitative treatment of JUV in exposure, dose-response, and risk calculations.

General approaches to issues (i) and (iv) have been reviewed (Bogen, 1995; NRC, 1994). Also pertaining to issues (i) and (iv) are proposed methods to extend quantitative 
probabilistic methods now commonly applied in cancer risk assessment to noncancer endpoints, which involve replacing traditional uncertainty/safety factors by corresponding empirically based, or reasonable default, probability distributions (Baird et al., 1996; Carlson-Lynch et al., 1999; Dourson et al., 1996; Lewis, 1993; Renwick, 1993; Slob and Pieters, 1998; Weil, 1972). Issue (ii) is a major focus of the proposed USEPA guidelines for carcinogen risk assessment (USEPA, 1996), but in this regard the USEPA recommends a non-quantitative, narrative approach that cannot possibly address issue (iv). Concerning issue (iii), a number of physiologically based pharmacokinetic (PBPK) models have been developed for TCE (Abbas and Fisher, 1997; Allen and Fisher, 1993; Fisher and Allen, 1993; Fisher et al., 1998; Stenner et al., 1998), and corresponding methods for efficient PBPK analysis have been developed under different mechanistic assumptions concerning TCE-induced cancer (Bogen, 1988; Bogen and Gold, 1997). Concerning issues (ii) and (iii), PBPK methods for TCE have been applied under alternative mechanistic assumptions (Fisher and Allen, 1993), but this has never been done in a way that integrates JUV information or efficient analytic (as opposed to numerical) PBPK-calculation methods.

In recently proposed revised methods for deriving Ambient Water Quality Criteria, the USEPA indicated that a goal of these methods should be to integrate cancer and noncancer assessments, and more specifically "to harmonize cancer and noncancer dose-response approaches and permit comparisons of cancer and noncancer risk estimates" (USEPA, 1998; p. 59,97). To the extent this goal was achieved, these proposed methods would provide guidance on how to address issues (i)-(iv) in a systematic probabilistic framework for risk assessment. While the proposed methods do address multiple (cancer and noncancer) endpoints and alternative (linear vs. nonlinear) mechanisms of carcinogenic action, they do not specifically facilitate or even address their stated goal of integrating cancer and noncancer dose-response methods to yield comparable or aggregate measures of risk. Furthermore, this goal is unnecessarily impeded by some of the proposed methods, including those that either: address dose-response differently for noncarcinogens vs. "nonlinear" carcinogens, address generic pharmacokinetic considerations differently for noncarcinogens vs. ("linear" or "nonlinear") carcinogens, consider non-ingestive exposure as well as human interindividual variability in dose-response for noncarcinogens and "nonlinear" 
carcinogens but not for "linear" carcinogens, or yield estimates of risk for "linear" carcinogens but do not for noncarcinogens and "nonlinear" carcinogens.

The impact of such inconsistencies on the problem of how to do unified risk assessment for cancer and noncancer endpoints is illustrated by the issue of whether or how to apply a toxicodynamic scaling factor to account for systematic interspecies differences in response as a function of biologically effective dose. For noncarcinogens, recently proposed USEPA methods include a good explanation of why, in the absence of relevant data, it is appropriate to apply two separate scaling factors (by default, each equal to a factor of 3 ) to account for interspecies toxicokinetic and toxicodynamic differences, respectively (USEPA, 1998; p. 140):

"The rationale for the use of PBPK models is that the pharmacokinetics and pharmacodynamics of a chemical each contribute to a chemical's observed toxicity, and specifically, to observed differences among species in sensitivity. Pharmacokinetics describes the absorption, distribution, metabolism, and elimination of chemicals in the body, while pharmacodynamics describes the toxic interaction of the agent with the target cell. In the absence of specific data on their relative contributions to the toxic effects observed in species, each is considered to account for approximately one half of the variability in observed effects, as is assumed in the development of RfCs and RfDs [i.e., reference concentrations and doses, respectively]. The implication of this assumption is that an interspecies uncertainty factor of 3 rather than 10 could be used for deriving an RfD when valid pharmacokinetic data and models can be applied ...."

For carcinogens, there is agreement that animal-to-human extrapolation of toxicokinetically equivalent effective dose may be accomplished by the use of an appropriate, validated PBPK model if one is available, and if not, by assuming that toxicokinetically equivalent doses scale proportional to body surface area or (body weight) ${ }^{0.75}$ (USEPA, 1992,1996,1998). However, federal policy concerning how/whether to apply an interspecies toxicodynamic scaling factor is not consistent. For example, the Health/Risk Assessment Committee of the Integrated Chlorinated Solvents Project (a committee comprised of representatives from four federal agencies) held that "it is strongly arguable that the surface-area correction is not a correction on dose to allow for pharmacokinetics, but rather a correction on risk to allow for many factors, including pharmacodynamics" (USEPA, 1987a; p. 125). For "linear" carcinogens, however, the USEPA has more recently proposed that no interspecies 
toxicodynamic scaling factor is required for carcinogens whenever a PBPK approach has been used to account for interspecies toxicokinetic differences (USEPA, 1998).

Likewise, interindividual variability in sensitivity/susceptibility per se to environmentally induced cancer is not typically considered in risk extrapolations for carcinogens assumed to have a genotoxic/linear-no-threshold mechanism of action (USEPA, 1996, 1998). In this respect, past practice has been to focus (implicitly) on risk to persons who have an average level of susceptibility, when there is no reason to predict that the exposed population is one that may reflect an unusual degree of hypersusceptibility to environmentally induced cancer (NRC, 1994). For noncarcinogens, however, a so-called "uncertainty" factor of up to 10 has traditionally been applied "to account for the variation in sensitivity (intraspecies variation) among the members of the human population"; and a similar factor was proposed recently by the USEPA for use with all "nonlinear" carcinogens (USEPA, 1998; p. 110,122). Confusion between uncertainty and interindividual variation also appears in proposed new approaches to model differences in human sensitivity by probabilistic methods rather than by the traditional use of "uncertainty" factors (Carlson-Lynch et al., 1999; Slob and Pieters, 1998). Because there is little doubt that substantial human variability exists in susceptibility to environmentally induced cancer (NRC, 1994), a truly systematic probabilistic framework to assessing risks pertaining to cancer and noncancer endpoints clearly requires a consistent approach to intraspecies variability in dose-response.

Recent proposals for so-called "unified" or "comprehensive" approaches to risk assessment for cancer and noncancer endpoints (Butterworth and Bogdanffy, 1999; Gaylor et al., 1999) fail to address the complete set of issues (i)-(iv) listed above. These proposals essentially recommend merely that a traditional safety-factor approach be used for cancer and noncancer endpoints alike; they focus on how to define exposure levels that protect against a single endpoint, rather than on how to calculate actual levels of aggregate risk for both cancer and noncancer endpoints. Therefore, no methods or studies exist that address the complete set of issues (i)-(iv) for integrated risk characterization. 


\subsection{Study Objectives}

This study (Phase 2 together with Phase 1) was designed to accomplish two overall objectives. The first overall objective was to provide to the U.S. Air Force and regulatory agencies new quantitative procedures that address JUV in exposure and dose-response assessment to better characterize potential health risk. Such methods could be used at sites where populations may now or in the future be faced with using groundwater contaminated with low concentrations of TCE. The second overall objective was to illustrate and explain the application of these procedures with respect to available data for TCE in ground water beneath an inactive landfill site that is undergoing remediation at Beale Air Force Base in California. The results of this case study are intended to illustrate how the more realistic and more meaningful risk estimates obtained using methods we describe compare to corresponding conservative risk estimates calculated using a traditional deterministic screening-level approach. Application of the methods developed in this project can lead to more reasonable and equitable risk-acceptability criteria for potentially exposed populations at specific sites.

The specific objective of the present report is to describe consistent and coherent methods devised to address issues (a)-(d) discussed in the previous subsection, and to report and discuss an application of these methods, together with other methods and information developed in Phase 1 of this project, to the specific problem of characterizing risk posed by TCE in ground water at Site LF-13 at Beale Air Force Base.

\section{METHODS}

Methods used to address joint uncertainty and interindividual variability (JUV) in risk posed by TCE contamination at Site LF-13 was calculated and characterized as described below in subsections pertaining to: (1) the systematic probabilistic framework adopted for this analysis, (2) TCE concentration and route-specific exposures, (3) corresponding biologically effective doses and related physiologically based pharmacokinetic (PBPK) considerations, (4) dose-response for cancer and noncancer endpoints, (5) characterization of joint uncertainty and interindividual variability (JUV) in risk as a function of JUV in input parameters relating to topics (2)-(4) listed above, and (6) data analysis and computation. 
Consistent with established JUV notation, an overbar (i.e., - ) here denotes expectation with respect to heterogeneous parameters only, angle brackets (i.e., \langle\rangle ) denote expectation with respect to uncertain parameters only, each subscripted $U$ denotes a corresponding purely uncertain variate, and each subscripted $V$ denotes a corresponding purely interindividually heterogeneous variate (Bogen and Spear, 1987; NRC, 1994; Bogen, 1995). The operator E (e.g., as in EX) is used to signify expectation with respect either to variability or to uncertainty, bot not to both (e.g., to either the variability-expectation $\bar{X}$, or to the uncertainty-expectation $\langle X\rangle$, with respect to a variate $X$ ). Other italic capital letters are used to denote (potentially) JUV variates. Also, each subscripted $K$ denotes a corresponding constant used below to estimate risk, $X_{\mathrm{MA}, \mathrm{P}}$ (in $\mathrm{mg} \mathrm{kg}^{-1} \mathrm{~d}^{-1}$ ) denotes a mechanistically relevant measure of TCE intake by the indicated exposure pathway $(\mathrm{P})$ that pertains to the indicated mechanism/mode of action (MA) for TCE-induced toxicity, and $D_{\mathrm{MA}, \mathrm{P}}$ denotes a corresponding biologically effective dose, where, for both $X$ and $D$, the subscript MA specifies either a genotoxic $(G)$ or cytotoxic (C) assumed mode of action, and the subscript $P$ indicates either an ingestion (ing), inhalation (inh), or dermal (der) exposure pathway. Additional related variates are defined below.

Some variates defined in Daniels et al. (2000), denoted as they were in that report, are also referred to below. All constants and variates defined in the present report used as inputs to estimate risk are defined below and are summarized in Table 2, which appears at the end of Methods (after Section 2.6) prior to Results (Section 3).

\subsection{Systematic Probabilistic Framework}

Health risk associated with residential exposure to TCE from ground water at Site LF-13 on Beale Air Force Base in California was analyzed using the systematic probabilistic framework summarized in Figure 1. Total associated risk, $R$, was defined as the increased individual lifetime probability of incurring a toxic (cancer and/or noncancer) endpoint due to TCE exposure from three pathways: direct ingestion of TCE-contaminated groundwater, dermal absorption of TCE while showering or bathing, and inhalation of TCE volatilized from water to household air. For volatile organic compounds (VOCs) such as TCE, these three pathways typically are the most significant contributors to total daily residential intake. Each route-specific TCE intake 
was converted to a corresponding biologically effective dose for each MA and toxic endpoint considered, where this conversion was made using efficient MA-specific forms of a human PBPK model. Route-specific effective doses were summed for each MA to obtain (two) measures of MA-specific total effective dose (see Section 2.2). As detailed in Section 2.4, two MAs were considered for TCE: a genotoxic MA (MA $\left.A_{G}\right)$ and a cytotoxic MA ( $\left(\mathrm{MA}_{\mathrm{C}}\right)$, with both MAs considered potentially relevant to cancer risk posed by TCE exposure, but only $\mathrm{MA}_{\mathrm{C}}$ considered relevant to noncancer risk posed by TCE exposure. Briefly, it was assumed that liver cytotoxicity is the most sensitive noncancer endpoint for TCE in humans based on the most sensitive experimental (mouse) data, that hepatotoxicity may (itself, or as the most sensitive available cytotoxic indicator) also explain and/or contribute to TCE-induced cancer observed in animal bioassays, and that genotoxicity may additionally explain and/or contribute to TCEinduced cancer observed in rodent bioassays. Increased likelihoods of cancer and of hepatotoxicity were each modeled as a MA-specific function of PBPK-based biologically effective dose in animals. Interspecies extrapolation of pharmacokinetic differences was obviated by consistent application of relevant PBPK models. Interspecies pharmacodynamic differences in dose-response were extrapolated using a single method applied to both cancer and noncancer endpoints. Intraspecies (interindividual) variability in human dose-response was modeled identically for both cancer and noncancer endpoints. Finally, increased risks of incurring either or both endpoints were estimated with respect to associated JUV, and these estimates were compared to corresponding traditional-type risk estimates obtained using deterministic methods.

The following subsections describe the specific methods used to apply the general approach just summarized, with respect to route-specific TCE exposures (Section 2.2), corresponding biologically effective doses (Section 2.3), dose-response for TCE-induced toxicity (Section 2.4), and unified risk characterization (Section 2.5).

\subsection{TCE Exposure}

Predicted rates $\left(X_{\mathrm{G}, \text { ing }}, X_{\mathrm{G}, \text { inh }}\right.$ and $\left.\mathrm{X}_{\mathrm{G}, \mathrm{der}} ; \mathrm{mg} \mathrm{kg}^{-1} \mathrm{~d}^{-1}\right)$ of route-specific, lifetime timeweighted average (LTWA) exposure to TCE due to Site LF-13 groundwater contamination at Beale AFB were calculated from corresponding rates $\left(E_{\text {Ing }} E_{\text {Inh }}\right.$ and $E_{\text {Derm }}$ ) and associated JUV defined in Equations 1-3 of the Phase-1 report (Daniels et al., 


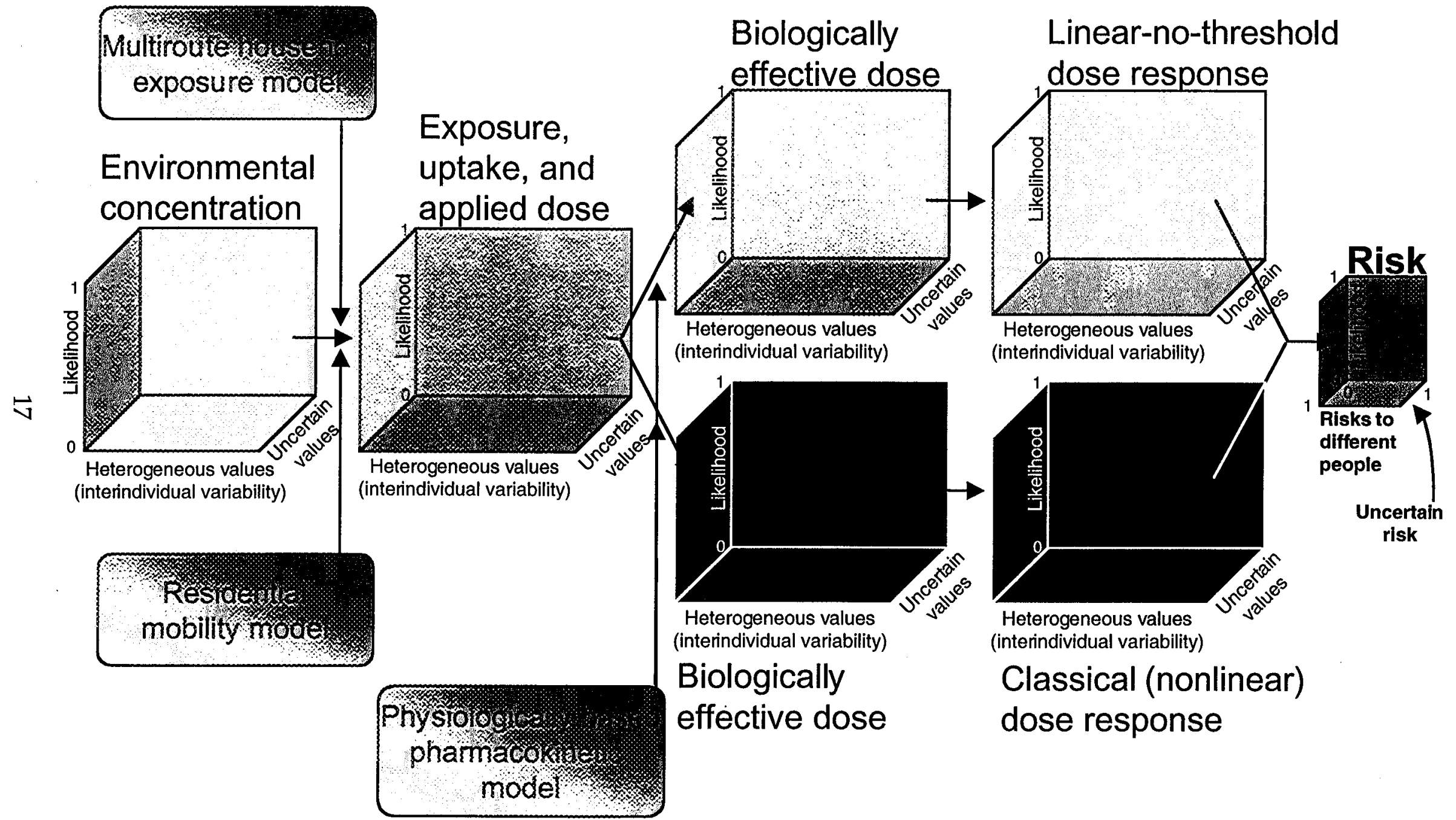

Figure 1. Unified probabilistic approach to risk assessment for TCE. 
1999). (Note that the Phase 1 report used slightly different subscript-notation.) Specifically, it was assumed that ,

$$
\begin{aligned}
& X_{\mathrm{G}, \text { ing }}=E_{\text {Ing }}, \\
& X_{\mathrm{G}, \text { der }}=E_{\text {Derm }} ; \\
& X_{\mathrm{G}, \text { inh }}=\left(V_{\mathrm{alvR}} / \operatorname{Inh}\right) E_{\text {Inh }}, \text { where }
\end{aligned}
$$

Inh $=$ total respiratory ventilation rate used in Daniels et al. $(2000)\left(\mathrm{m}^{3} \mathrm{~kg}^{-1} \mathrm{~d}^{-1}\right)$; and

$$
V_{\text {alvR }}=\frac{V_{\text {alvr }}}{1000 \mathrm{~L} \mathrm{~m}^{-3}} V_{\mathrm{alv}}=\frac{12.9 \mathrm{~L} \mathrm{~h}^{-1}}{1000 \mathrm{~L} \mathrm{~m}^{-3}}\left(V_{\mathrm{w}} / \mathrm{kg}\right)^{0.74-1} V_{\mathrm{alv}} \text {, in which: }
$$

$V_{\text {alvR }}=$ weight-normalized ventilation rate used in present study $\left(\mathrm{m}^{3} \mathrm{~h}^{-1}\right)$,

$V_{\text {alvr }}=$ non-normalized ventilation rate $\left(\mathrm{L} \mathrm{h}^{-1}\right)$,

$V_{\mathrm{W}}=$ body weight $(\mathrm{kg})$, and

$V_{\mathrm{alv}}=$ normalized interindividual variability in $V_{\mathrm{alvR}}$ that is independent of variability in $V_{\mathrm{W}}$ (unitless).

Equation 2 is an adaptation of the alveolar ventilation rate $V_{\text {alvr }}$ defined in a validated PBPK model for TCE in humans (Allen and Fisher, 1993). Because, as explained in Section 2.3, this PBPK model was integrated into the probabilistic framework used in the present study, $X_{\mathrm{G} \text {,inh }}$ was defined in terms of $V_{\text {alvR }}$ rather than the total ventilation rate Inh used by Daniels et al. (2000).

Variability in $V_{\mathrm{W}}$ for U.S. adults was modeled as approximately lognormal (LN) with an arithmetic mean (AM) of $71.0 \mathrm{~kg}$, standard deviation (SD) of $15.9 \mathrm{~kg}$, and corresponding coefficient of variation ( $\mathrm{CV}=\mathrm{SD} / \mathrm{AM}$ ) of 0.224 (CalEPA, 1996; Finley et al., 1994). Based on the method of moments (Aitchison and Brown, 1957) explained and developed in Appendix 1, the assumed AM and SD of $V_{\mathrm{W}}$ imply that $V_{\mathrm{W}} \sim \mathrm{LN}(4.24$, $0.221)$.

Weight-normalized rates of total respiratory ventilation for U.S. adults are approximately lognormally distributed with a CV of $\sim 0.3$ (CalEPA, 1996). As implied by Equation 1, the non-normalized alveolar ventilation rate $V_{\mathrm{alvr}}\left(\mathrm{L} \mathrm{h}^{-1}\right)$ is approximated as

$$
V_{\text {alvr }} \approx\left(12.9 \mathrm{~L} \mathrm{~h}^{-1}\right)\left(V_{\mathrm{w}} \mathrm{kg}^{-1}\right)^{-0.3} V_{\mathrm{alv}}
$$


The alveolar proportion of total lung volume was assumed to be nearly constant, and consequently variability in $V_{\text {alv }}$ was modeled as LN with an AM of 1 . Based on the method of moments (Appendix 1), it follows that $V_{\text {alv }} \sim \mathrm{LN}(-0.0409,0.286)$. To facilitate PBPK analyses described in Section 2.3, it was assumed that $V_{\text {alv }}, V_{\text {alvr }}$ and $V_{\text {alvR }}$ pertain to children as well as to adults.

Predicted daily (non-LTWA) peak TCE exposures $\left(X_{C, P}\right.$, in $\left.\mathrm{mg} \mathrm{kg}^{-1} \mathrm{~d}^{-1}\right)$ due to Site LF-13 groundwater contamination at Beale AFB were defined for ingestion and dermal exposure routes as follows, in terms of $X_{G, P}$ defined above (Equations 1a-b) and in terms of the constants $E F$ and $A T$ and the variate $E D$ defined in Equation 1 of the Phase-1 report (Daniels et al., 2000):

$$
\begin{aligned}
& X_{\mathrm{C}, \text { ing }}=[A T /(E D \times E F)] X_{\mathrm{G}, \text { ing }} \text {, and } \\
& X_{C, \text { der }}=[A T /(E D \times E F)] X_{\mathrm{G}, \text { der }} \text {, where }
\end{aligned}
$$

$E D$ = household exposure/residence duration used in Daniels et al. (2000) (y);

$E F=$ exposure frequency used in Daniels et al. (2000) $\left(\mathrm{d} \mathrm{y}^{-1}\right)$;

$A T=$ averaging time used in Daniels et al. (2000) corresponding to a 70-y exposure (d).

Predicted daily peak respiratory TCE exposure, $X_{C, \text { inh }}$, was similarly related to $X_{G, \text { inh }}$ except that $E_{\text {Inh }}$ (see Equation 1c) was defined by Daniels et al. (2000) to refer to total household LTWA exposure, whereas $X_{\mathrm{C} \text {,inh }}$ pertains to peak respiratory TCE exposure, which is assumed to occur during showering (and without reference to non-shower respiratory TCE exposures). Therefore, $X_{\mathrm{C} \text {,inh }}$ was modeled as follows based on the method of Daniels et al. (2000) as adapted in Equation 1c, but solely with reference to shower-related TCE exposure:

$$
X_{\mathrm{C}, \text { inh }}=V_{\mathrm{alvr}}\left(\frac{W_{\mathrm{sh}} \phi_{\mathrm{TCE}-\mathrm{sh}}}{1000 \mathrm{~L} \mathrm{~m}^{-3} \times A E_{\mathrm{sh}}}\right) C_{\mathrm{w}} \frac{V_{t, \text { inh }}}{1 \mathrm{~d}} \text {, where }
$$

$W_{\mathrm{sh}}=$ water-usage rate per person for shower $\left(\mathrm{L} \mathrm{h}^{-1}\right)$;

$\phi_{\mathrm{TCE}-\mathrm{sh}}=$ water-to-air transfer efficiency of TCE in the shower (unitless);

$A E_{\text {sh }}=$ air-exchange rate in the shower or bath stall $\left(\mathrm{m}^{3} \mathrm{~h}^{-1}\right)$;

$C_{\mathrm{w}}=$ TCE concentration in ground water $\left(\mathrm{mg} \mathrm{L}^{-1}\right)$; and 
$V_{t, \text { inh }}=\left(E T_{\text {sh }} \times 1 \mathrm{~d}\right)=$ shower duration $(\mathrm{h}) ;$

where variability in $W_{\mathrm{sh}}, \phi_{\mathrm{TCE}-\mathrm{sh}}, A E_{\mathrm{sh}}$ and $E T_{\mathrm{sh}}$, and uncertainty in $C_{\mathrm{w}}$, were all modeled as previously described (Daniels et al., 2000).

\subsection{Biologically Effective Dose}

For reasons discussed in Section 1.3, liver was assumed to model susceptible target tissue for TCE-induced cancer based on mouse bioassay data, and mouse hepatocellular toxicity was used to model the most sensitive TCE-induced noncancer (but possibly cancer-related) endpoint. Dose-response relations for TCE-induced endpoints were treated as functions of corresponding mechanism- and route-specific measures of biologically effective dose $D_{\mathrm{MA}, \mathrm{P}}\left(\mathrm{mg} \mathrm{kg}^{-1} \mathrm{~d}^{-1}\right)$ defined below. As indicated in Figure 1, PBPK and associated JUV models used to define $D_{\mathrm{MA}, \mathrm{P}}$ as functions of corresponding TCE exposures $\left(X_{\mathrm{MA}, \mathrm{P}}\right)$ were treated differently in view of uncertainty as to the extent to which the MA for TCE involves genotoxic $(G)$ processes with a plausibly linear doseresponse vs. cytotoxic/mitogenic $(C)$ processes with a likely nonlinear dose-response. To facilitate subsequent calculations, the following related quantities were also calculated:

$$
\begin{array}{lll}
D_{\mathrm{MA}} & =\sum_{i=\text { (ing,inh,der) }} D_{\mathrm{MA}, i}, \\
f_{\mathrm{MA}, \mathrm{P}} & =\left\langle\overline{D_{\mathrm{MA}, \mathrm{P}}}\right\rangle /\left\langle\overline{D_{\mathrm{MA}}}\right\rangle, & \\
B_{\mathrm{MA}, \mathrm{P}} & \left.=D_{\mathrm{MA}, \mathrm{P}} / \overline{D_{\mathrm{MA}, \mathrm{P}}}\right\rangle, & \text { and } \\
B_{\mathrm{MA}} & =\sum_{i=\text { (ing,inh,der) }} \frac{B_{\mathrm{MA}, i}}{3} \mid\left(F_{B_{\mathrm{MA}, i}}\left(B_{\mathrm{MA}, i}\right)=p\right), \text { for } 0 \leq p \leq 1, \quad \text { where }
\end{array}
$$

$D_{\mathrm{MA}}=$ total of all pathway-specific biologically effective doses under mechanistic assumption $M A\left(m g \mathrm{~g}^{-1} \mathrm{~d}^{-1}\right.$ for $M A=G, m g / L$ for $\left.M A=C\right)$;

$B_{\mathrm{MA}, \mathrm{P}}=$ normalized biologically effective dose for pathway $\mathrm{P}$ under mechanistic assumption MA (unitless);

$B_{\mathrm{MA}}=$ mean value of the cumulative probability distribution functions (cdfs) corresponding to all pathway-specific normalized biologically effective doses under mechanistic assumption MA (unitless); and

$f_{\mathrm{MA}, \mathrm{P}}=$ fraction of $\left\langle\overline{D_{\mathrm{MA}}}\right\rangle$ due to pathway $\mathrm{P}$ (unitless). 
The variate $B_{\mathrm{MA}}$ is defined (by Equation $5 \mathrm{~d}$ ) to take advantage of the fact that MAspecific $B_{\mathrm{MA}, \mathrm{P}}$ distributions were found to be nearly identical for all pathways $\mathrm{P}$ in this case study (see Results). Therefore, in calculations implementing Monte Carlo simulations done in this study, $\mathrm{E} B_{\mathrm{MA}}$ distributions were used in place of the greater number of corresponding $\mathrm{E} D_{\mathrm{MA}, \mathrm{P}}$ distributions (see Section 2.6). The order of (uncertainty- vs. variability) expectation operations in Equations 5a-b is arbitrary in this case study, because the order was not found to have a substantial effect on the value of $\left\langle\overline{D_{\mathrm{MA}}}\right\rangle$ obtained (due principally to the linear structure and behavior of the models used for $D_{\mathrm{MA}, \mathrm{P}}$ previously described; see Daniels et al., 2000). Consequently, $\left\langle\overline{B_{\mathrm{MA}}}\right\rangle=\overline{\left\langle B_{\mathrm{MA}}\right\rangle}=1$ by definition. Note also that, because of the multiplicative model structures implied by Equations $4 \mathrm{a}-\mathrm{c}, B_{\mathrm{MA}}=\left\langle B_{\mathrm{MA}}\right\rangle \overline{B_{\mathrm{MA}}}$.

The rationale for including both genotoxic and cytotoxic MAs into this analysis is discussed below, followed by subsections detailing PBPK models and methods used to calculate corresponding biologically effective genotoxic and cytotoxic doses to bioassay animals and to humans.

\subsubsection{Uncertainty in mechanism of toxic action}

As discussed in Section 1.3, there is fundamental "model" uncertainty regarding critical mechanism(s) explaining the observed ability of TCE to increase tumor incidence in rodent bioassays and its suspected ability to do the same in humans. This uncertainty can be represented by the following four alternative mechanistic assumptions (MAs):

Assumption $1\left(\mathrm{MA}_{\mathrm{G}}\right)$ is the traditional approach to assessing TCE cancer risk, which presumes that TCE increases cancer risk only via one or more genotoxic mechanisms of action, involving DNA damage that is linearly proportional to the biologically effective concentration of one or more of TCE's reactive metabolites (Bogen, 1988; Bogen et al., 1988; Brown et al., 1990; USEPA, 1985,1987a).

Assumption 2 ( $\mathbf{M A}_{\mathrm{C}}$ ) is that observed TCE-induced (e.g., liver) cancer is due entirely to increased net proliferation of spontaneous premalignant cells elicited primarily by TCA, by a cytotoxic and/or perhaps a directly mitogenic mechanism (Andersen et al., 1998; Bogen and Gold, 1997).

Assumption 3 ( $\mathbf{A}_{\mathrm{GnC}}$ ) is the composite assumption that both genotoxic and nongenotoxic mechanisms contribute to observed TCE carcinogenicity in 
bioassays, i.e., that both $\mathrm{MA}_{\mathrm{G}}$ and $\mathrm{MA}_{\mathrm{C}}$ are true. However, to the extent $\mathrm{MA}_{\mathrm{G} C \mathrm{C}}$ is true, uncertainty remains as to the quantitative role played by each mechanism involved. This kind of uncertainty is often referred to as "parameter" uncertainty, because it is possible to reflect this as uncertainty pertaining to a single parameter (in a sufficiently general model) that governs the weight to be given to each of the two mechanisms considered to be operative under $\mathrm{MA}_{\mathrm{G} n \mathrm{C}}$.

Assumption 4 ( $\mathbf{M A}_{\mathrm{GuC}}$ ) is the "dichotomous" assumption that either $\mathrm{MA}_{\mathrm{G}}$ or $\mathrm{MA}_{C}$ is true, but there is "model" uncertainty as to which one of these possibilities is true, in view of the fact that the "parameter" uncertainty discussed above in reference to $\mathrm{MA}_{\mathrm{G} \cap \mathrm{C}}$ is quantitatively equivalent to "model" uncertainty.

In view of evidence discussed in Section 1.4 supporting the plausibility of both $\mathrm{MA}_{\mathrm{G}}$ and $\mathrm{MA}_{C}$, both of these mechanistic assumptions were used to define route-specific biologically effective dose and dose-response for TCE-induced cancer. Of course, $\mathrm{MA}_{C}$ was used exclusively as the basis for calculating biologically effective dose and doseresponse for TCE-induced noncancer endpoints. Below, methods used to estimate biologically effective doses corresponding to mechanisms $M A_{G}$ and $M A_{C}$ are described, following an explanation of the PBPK modeling approach that was adopted in this study to accommodate both mechanisms.

\subsubsection{PBPK modeling approach}

A number of multi-compartment PBPK models have been developed that provide reasonably well-validated descriptions of the uptake, distribution, metabolism, and excretion of TCE administered by various routes to mice, rats and humans (Abbas and Fisher, 1997; Allen and Fisher, 1993; Bogen, 1988; Fisher and Allen, 1993; Fisher et al., 1991,1998; Stenner et al., 1998; USEPA, 1985, 1987b). In contrast to earlier PBPK models describing TCE distribution, metabolism and excretion using four physiological compartments, the more recent "second generation" models include additional compartments to describe distribution, metabolism and excretion of TCA and of unbound and glucuronide-bound trichloroethanol in mice and humans (Abbas and Fisher, 1997; Fisher et al., 1998), and to account for enterohepatic recirculation of TCA and of trichloroethanol-glucuronide (Stenner et al., 1998). Although the newer PBPK models are more realistic, they are less convenient to incorporate into the adopted probabilistic framework relative to earlier-type 4-compartment models. It is also not apparent that any improved ability to fit empirical data used to validate the newer vs. 
the earlier models implies any corresponding substantial improvement in the specific measures of biologically effective dose discussed below, namely, total metabolized TCE and peak plasma concentration of TCA. Indeed, an earlier-type 4-compartment model for TCE in humans appears to provide fairly accurate predictions of the peak value, $\operatorname{Max}\left(C_{\mathrm{TCA}}\right)$, of TCA concentration in plasma measured in several different studies involving humans exposed by inhalation to various air concentrations of TCE (Allen and Fisher, 1993), whereas a corresponding "second generation" model appears to underpredict $\operatorname{Max}\left(C_{\mathrm{TCA}}\right)$ by up to $\sim 40 \%$ in human subjects exposed to 50 or $100 \mathrm{ppm}$ TCE in air (Figure 8 of Stenner et al., 1998). Therefore, earlier-type 4-compartment models (Allen and Fisher, 1993; Bogen, 1988) were used for PBPK-based calculations of biologically effective dose in the present study, as described below. However, recently reported experimental data on human variability in key PBPK parameter values (Fisher et al., 1998; Lipscomb et al., 1998) was incorporated into the present analysis as discussed below.

\subsubsection{Effective genotoxic dose}

Under $\mathrm{MA}_{\mathrm{G}}$ for TCE (i.e., assuming that TCE is a "linear"/genotoxic carcinogen), bioassay-based potency traditionally has been expressed as increased risk per unit of PBPK-estimated total LTWA metabolized TCE per kg body weight per day, without accounting for PBPK-related uncertainty and variability (Bogen, 1988; Brown et al., 1990; USEPA, 1985, 1987b). There is an indication this policy will likely persist (USEPA, 1996). Measures of biologically effective dose, as LTWA metabolized TCE in $\mathrm{mg} \mathrm{kg}^{-1} \mathrm{~d}^{-1}$ to animals in bioassays positive for TCE-induced liver or kidney cancer, were obtained from Table 4 of Bogen (1988). Similar measures of route-specific biologically effective dose $D_{\mathrm{G}, \mathrm{P}}$ to humans under $\mathrm{MA}_{\mathrm{G}}$ were used for the present analysis, namely:

$$
D_{\mathrm{G}, \mathrm{P}}=V_{\mathrm{fm}, \mathrm{P}} X_{\mathrm{G}, \mathrm{P}}, \quad \text { for } \mathrm{P}=\text { ing, inh, or der, where }
$$

$V_{\mathrm{fm}, \mathrm{P}}=$ limiting fraction of total TCE intake by pathway $\mathrm{P}$ that is metabolized conditional on intake being sufficiently small to ensure that saturation of TCE metabolism remains negligible (unitless).

For multi-compartment PBPK models like that of Allen and Fischer (1993), these limiting metabolized fractions were shown previously to be 


$$
\begin{aligned}
V_{\mathrm{fm}, \text { ing }}=\left[1+\frac{K_{\mathrm{m}}}{V_{\mathrm{max}}}\left(\frac{V_{\mathrm{pb}}}{V_{\mathrm{alvr}}}+\frac{1}{V_{\mathrm{liv}}}\right)^{-1}\right]^{-1}, \text { and } \\
V_{\mathrm{fm}, \text { inh }}=V_{\mathrm{fm}, \mathrm{der}}=\left[1+\frac{V_{\mathrm{alvr}}}{V_{\mathrm{Pb}}}\left(\frac{K_{\mathrm{m}}}{V_{\max }}+\frac{1}{V_{\mathrm{liv}}}\right)\right]^{-1}, \text { where }
\end{aligned}
$$

$V_{\text {alvr }}=$ alveolar ventilation rate (defined in Equations 2 and 3) $\left(\mathrm{L} \mathrm{h}^{-1}\right)$;

$V_{\text {liv }}=$ the rate of blood perfusion to liver $\left(\mathrm{L} \mathrm{h}^{-1}\right)$;

$V_{\mathrm{Pb}}=$ the blood:air partition coefficient for TCE $\left(\mathrm{L}_{\mathrm{air}} \mathrm{L}_{\text {blood }}^{-1}\right)$;

$V_{\max }=$ maximum rate of TCE metabolism $\left(\mathrm{mg} \mathrm{h}^{-1}\right) ;$

$K_{\mathrm{m}}=$ Michaelis-Menten affinity/saturation constant $\left(\mathrm{mg} \mathrm{L}^{-1}\right)$; and

where $V_{\text {alvr }}$ is alveolar ventilation rate defined above (Equation 3), and where, for Michaelis-Menten parameters $K_{\mathrm{m}}$ and $V_{\max }$ assumed to govern metabolic saturation kinetics for TCE, the mass unit (mg) refers to TCE and the volume (L) to venous blood exiting liver (Bogen, 1988; Bogen and Hall, 1989).

To derive human biologically effective doses under $\mathrm{MA}_{\mathrm{G}}$ (as well as under $\mathrm{MA}_{C}$, as explained below), Equations 7a-b were applied assuming: $V_{\text {alvr }}$ is defined by Equation 2, $V_{\text {liv }}=26 \% \times(15.0 / 12.9) \times V_{\text {alv }}$ (Allen and Fisher, 1993), $K_{\mathrm{m}}=1.5 \mathrm{mg} \mathrm{L}^{-1}$ (i.e., treated as a constant) (Allen and Fisher, 1993), and that $V_{\mathrm{Pb}} \sim \mathrm{N}(10.2,1.6)$ for males and females combined (Fisher et al., 1998). Variability in the maximal rate of TCE metabolism, $V_{\max }$, was modeled as $\mathrm{LN}$ with

$$
V_{\max }=\left(14.9 \mathrm{mg} \mathrm{h}^{-1}\right)\left(V_{\mathrm{W}} \mathrm{kg}^{-1}\right)^{-0.3} V_{\mathrm{V} \max },
$$

which adapts the definition used by Allen and Fisher (1993) to incorporate a multiplicative factor $V_{\mathrm{V} \max }$ reflecting $V_{\max }$-related variability, where $V_{\mathrm{V} \max }$ was assumed to have an $\mathrm{AM}$ of 1 . Under these assumptions, Equations 7a-b are simplified to:

$$
\begin{aligned}
V_{\mathrm{fm}, \text { ing }} & =\left\{1+V_{\mathrm{alv}}\left[V_{\mathrm{V} \max }\left(0.7700 V_{\mathrm{Pb}}+2.547\right)\right]^{-1}\right\}^{-1}, \text { and } \\
V_{\mathrm{fm}, \text { inh }}=V_{\mathrm{ff}, \text { der }} & =\left[1+\frac{V_{\mathrm{alv}}}{V_{\mathrm{Pb}}}\left(\frac{1.299}{V_{\mathrm{V} \max }}+3.307\right)\right]^{-1},
\end{aligned}
$$


in which no more than three significant figures are implied. From Equation $9 b$ it is clear that $V_{\mathrm{fm}, \text { inh }}$ is correlated with $V_{\mathrm{alv}}$. From Equation 1c, it follows that this correlation is implied in Equation 6 defining $D_{\mathrm{G} \text {,inh }}$, as well as in Equation 15b below (in Section 2.3.4) that defines the corresponding cytotoxic dose $D_{\mathrm{C}, \text { inh }}$. Note, however, that the limiting metabolized fractions defined by Equations $9 \mathrm{a}-\mathrm{b}$ are independent of body weight $\left(V_{\mathrm{W}}\right)$, and thus are independent of $D_{\mathrm{MA}, \mathrm{P}}$ for $\mathrm{P}=\{$ ing, der $\}$ defined by Equation 6 (and by Equations 15a-b below).

Based on in vitro measures of $V_{\max }$ for TCE using human microsomes and hepatocytes sampled from 4 to 6 different donors (Lipscomb et al., 1998), the CV of $V_{\max }$ was estimated to be approximately 0.60 . Based on the method of moments (Appendix 1), and conditional on assumed variability in $V_{\mathrm{W}}$ discussed above (after Equation 2), the latter $\mathrm{CV}$ estimate implies that $V_{\max } \sim \mathrm{LN}(-0.152,0.551)$. Systematic uncertainties pertaining to $V_{\mathrm{fm}, \mathrm{P}}$ are likely to be small relative to the combined effect of interindividual pharmacokinetic variabilities, so uncertainty per se is not incorporated into Equations $9 \mathrm{a}-\mathrm{b}$ used to define $V_{\mathrm{fm}, \mathrm{P}}$.

Note that, conditional on the adopted PBPK model, Equations 7a-b and 9a-b remain true regardless of any (dynamic or static) pattern of exposure(s) involved, provided that metabolism remains virtually unsaturated, which in turn ensures that the corresponding system of linked ordinary differential equations remains linear (Bogen, 1988; Bogen and Spear, 1987).

\subsubsection{Effective cytotoxic dose}

Under $\mathrm{MA}_{\mathrm{C}}$ for TCE, hepatocellular oxidative damage is assumed to comprise or elicit premalignant liver-cell proliferation and consequent increased tumor risk in mice, and is further assumed to correlate best with the daily peak value $\operatorname{Max}\left(C_{\mathrm{TCA}}\right)$, of TCA concentration in plasma, rather than with LTWA total metabolized TCE or related areas under concentration-times-time curves for blood or other tissues (Bogen and Gold, 1997). Similar reliance on peak rather than LTWA metabolic yield was used for $\mathrm{MA}_{C}$-based risk assessment for chlorinated methanes, based on empirical evidence supporting the former measure as the best predictor of oxidative damage (Bogen, 1990a). In the absence of dose-response data on TCA-induced rodent nephrotoxicity, and consistent with information discussed in Section 2.4, $\operatorname{Max}\left(C_{\mathrm{TCA}}\right)$ was also taken to be 
the biologically effective cytotoxic dose for potential TCE-induced kidney cancer under $\mathrm{MA}_{\mathrm{C}}$. It was further assumed that $\operatorname{Max}\left(C_{\mathrm{TCA}}\right)$ is the biologically effective cytotoxic dose for TCE-induced noncancer endpoints, so in general it was assumed that $D_{\mathrm{C}, \mathrm{P}}$ for any exposure pathway $\mathrm{P}$ is the value of $\operatorname{Max}\left(C_{\mathrm{TCA}}\right)$ produced in response to a corresponding exposure $X_{C, P}$ defined in Equation 4a-c. Corresponding total effective dose $\left(D_{C}\right)$ was (in Equation 5a) defined as the sum of $D_{C, P}$ from all exposure pathways, as discussed below following Equation 13b.

In the context of low-dose risk extrapolation for humans based on the PBPK model for TCE used here, all saturable (Michaelis-Menten) PBPK relations linearize. Therefore, this PBPK model was evaluated using an entirely analytic approach previously described (Bogen and Gold, 1997), which is simpler yet equivalent to alternative, relatively cumbersome numerical methods more commonly applied. By this approach (see Equation 4 of Bogen and Gold, 1997),

$$
\frac{\mathrm{d} C_{\mathrm{TCA}}(t)}{\mathrm{d} t}=\frac{K_{\mathrm{fTCA}} K_{\mathrm{MW}}}{V_{\mathrm{W}} V_{\mathrm{fd}}}\left(\frac{C_{\mathrm{TCE}}(t) V_{\max }}{C_{\mathrm{TCE}}(t)+K_{\mathrm{m}}}\right)-V_{\mathrm{ke}} C_{\mathrm{TCA}}(t), \quad \text { where }
$$

$C_{\mathrm{TCA}}(t)=$ concentration at time $t$ of TCA in plasma $\left(\mathrm{mg} \mathrm{L}^{-1}\right)$;

$C_{\mathrm{TCE}}(t)=$ concentration at time $t$ of TCE in venous blood exiting liver $\left(\mathrm{mg} \mathrm{L}^{-1}\right)$;

$K_{\text {fTCA }}=$ net effective fraction of total TCE intake metabolized to TCA (unitless);

$K_{\mathrm{MW}}=\mathrm{TCA}$ to TCE molecular-weight ratio (unitless); and

$V_{\mathrm{fd}}=$ fraction of body weight corresponding to apparent volume of distribution for TCA $\left(\mathrm{L} \mathrm{kg}^{-1}\right)$; and

$V_{\mathrm{ke}}=$ first-order rate constant for elimination of TCA from plasma $\left(\mathrm{h}^{-1}\right)$;

and where $V_{\mathrm{w}}, V_{\max }$ and $K_{\mathrm{m}}$ were defined above (after Equations $2,7 \mathrm{~b}$, and $7 \mathrm{~b}$, respectively). It was assumed that $K_{\mathrm{fTCA}}=0.33$ (Allen and Fisher, 1993), and the ratio $K_{\mathrm{MW}}$ is 1.228 (see Bogen and Gold, 1997).

Conditional on any regular pattern of peak daily TCE exposures $X_{C, P}$ that - by any pathway $\mathrm{P}$ and corresponding duration $V_{t, \mathrm{P}}$-are small enough to ensure that $C_{\mathrm{TCE}}(t)<<$ $K_{\mathrm{m}}$ for all $t$, Equation 10 implies that $C_{\mathrm{TCA}}(t)$ attains a dynamic equilibrium in which

$$
\operatorname{Max}\left[C_{\mathrm{TCA}}(t)\right]=D_{\mathrm{C}, \mathrm{P}}=\frac{K_{\mathrm{fTCA}} K_{\mathrm{MW}} V_{\mathrm{fm}, \mathrm{P}}}{V_{\mathrm{fd}} V_{\mathrm{ke}}}\left(X_{\mathrm{C}, \mathrm{P}} \times 1 \mathrm{~d}\right)\left[\frac{f_{\mathrm{deq}}}{V_{t, \mathrm{P}}}\right], \text { where }
$$




$$
f_{\text {deq }}=\frac{1-\exp \left(-V_{\mathrm{ke}} V_{t, \mathrm{P}}\right)}{1-\exp \left(-V_{\mathrm{ke}} \times 24 \mathrm{~h}\right)}
$$

$V_{t, \mathrm{P}}=$ duration $(\leq 24 \mathrm{~h})$ of peak daily exposure $\mathrm{X}_{\mathrm{C}, \mathrm{P}}$ by pathway $\mathrm{P}(\mathrm{h})$; and

$f_{\text {deq }}=$ fraction of $\operatorname{Max}\left[C_{\mathrm{TCA}}(t)\right]$ conditional on a hypothetical infinite exposure duration that is attained at dynamic equilibrium conditional on $V_{t, \mathrm{p}} ;$

in which $X_{\mathrm{C}, \mathrm{P}}$ and $V_{t, \text { inh }}$ were defined via Equations $4 \mathrm{a}-\mathrm{c}, V_{\mathrm{fm}, \mathrm{P}}$ was defined in Equations $9 \mathrm{a}-\mathrm{b}$, and all other variates in Equations 11-12 (i.e., besides $f_{\text {deq }}, V_{t, \mathrm{P}}$, and $X_{\mathrm{C}, \mathrm{P}}$ ) were defined following Equation 10. Equation 11 is a multi-route generalization of Equations 6 and 7 of Bogen and Gold (1997). Figure 2 shows how the bracketed term in Equation 11 is well approximated by

$$
\begin{aligned}
& {\left[f_{\text {deq }} / V_{t, \mathrm{P}}\right] \approx(24 \mathrm{~h})^{-1}+0.5053 V_{\mathrm{ke}}+(1.661 \mathrm{~h}) V_{\mathrm{ke}}^{2}} \\
& \text { for } V_{t, \mathrm{P}} \leq 0.5 \mathrm{~h} \text { and } V_{\mathrm{ke}} \leq 0.1 \mathrm{~h}^{-1} \\
& \approx(24 \mathrm{~h})^{-1}+0.5053 V_{\mathrm{ke}} \quad \text { for } V_{t, \mathrm{P}} \leq 0.5 \mathrm{~h} \text { and } V_{\mathrm{ke}} \leq 0.04 \mathrm{~h}^{-1} \text {, }
\end{aligned}
$$

in which no more than three significant figures are implied.

As indicated following Equation $4 \mathrm{c}$, variability in $V_{t, \text { inh }}$ was modeled as $\mathrm{LN}$ with $V_{t, \text { inh }} \sim \mathrm{LN}(\ln 0.120, \ln 1.47)$ as previously described (Daniels et al., 2000), implying shower (or, more generally, bathing-related water-flow) durations that virtually never $\left(\mathrm{p}<10^{-11}\right.$ ) exceed $0.5 \mathrm{~h}$. It was further assumed that $V_{t, \text { der }}=V_{t, \text { inh }}$ as previously described (Daniels et al., 2000), that $V_{t, \text { ing }}<0.5 \mathrm{~h}$, and that $V_{t, \text { ing }}, V_{t, \text { inh }}$ and $V_{t, \text { der }}$ are timed such that the total effective cytotoxic exposure, $D_{\mathrm{C}}$ (defined by Equation $5 \mathrm{a}$ ), is maximized, so as to reflect the peak value of $\operatorname{Max}\left[C_{\mathrm{TCA}}(t)\right]$ predicted during a lifetime of different pathway-specific effective-exposure scenarios.

Based on methods used and data reported by Allen and Fisher (1993), variability in $V_{\text {fd }}$ was modeled as uniformly distributed between 5.2 and $15.2 \%$, and as being negatively correlated with $V_{\mathrm{W}}$ with a rank correlation coefficient of $\rho_{\mathrm{r}}\left(V_{\mathrm{fd}}, V_{\mathrm{W}}\right)=-0.50$; and it was assumed that

$$
V_{\mathrm{ke}}=0.028 V_{\mathrm{w}}^{-0.3} V_{\mathrm{e}}, \text { where }
$$

$V_{\mathrm{e}}=$ normalized variability in $V_{\mathrm{ke}}$ that is independent of variability in $V_{\mathrm{W}}$ (unitless), 


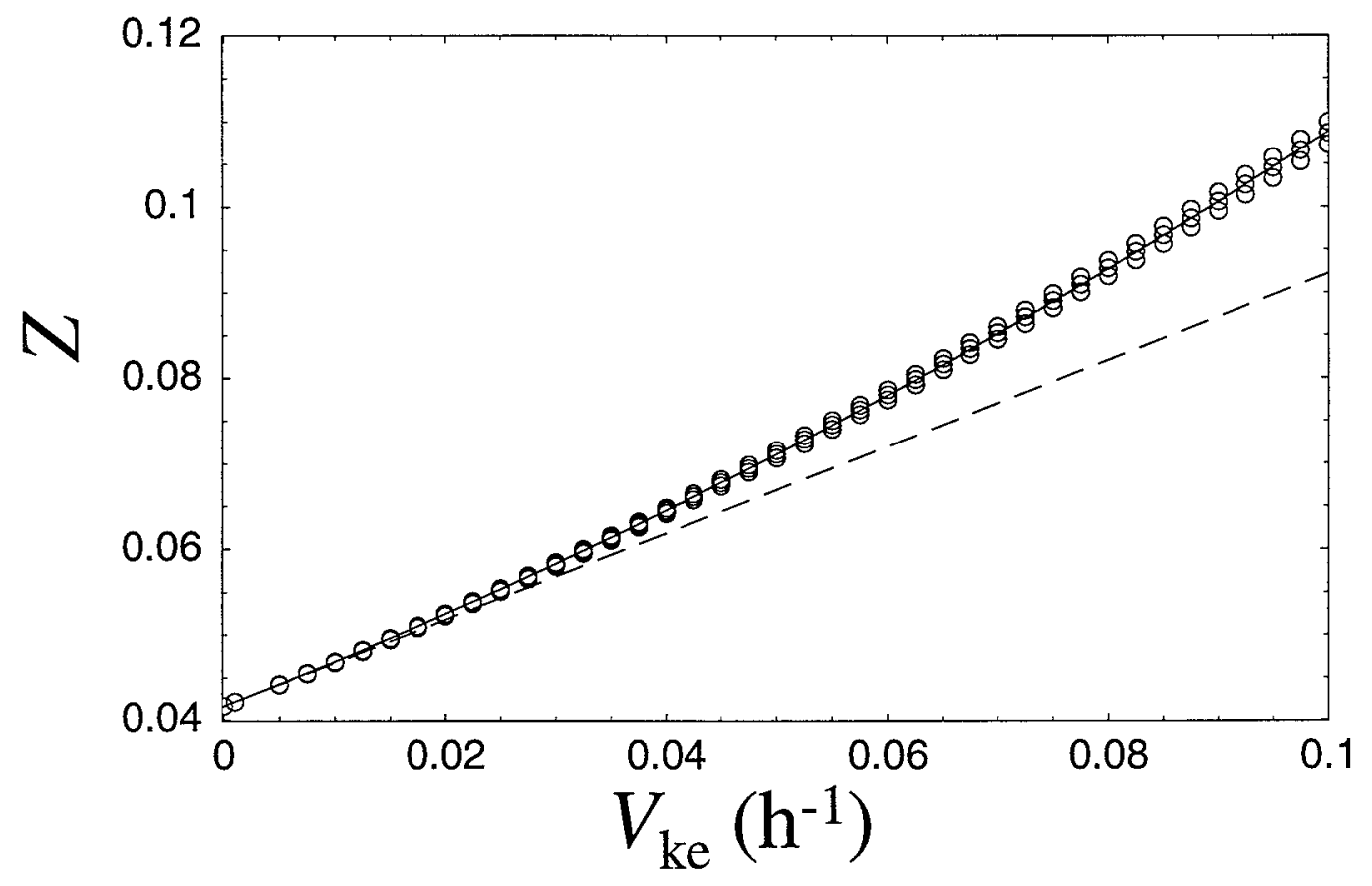

Figure 2. Approximation of the ratio $Z=\left(f_{\text {deq }} / V_{t, \mathrm{P}}\right)$, i.e., the fraction of steady-state that is attained under dynamic-equilibrium exposure conditions, divided by the duration $V_{t, \mathrm{P}}$ of daily exposure pulses. $Z$ (unitless) is plotted (using open points) as a function of 40 values of the (heterogeneous) TCA-elimination rate, $V_{\mathrm{ke}}$ evenly spaced between 0 and $0.1 \mathrm{~h}^{-1}$. The relatively small amount of vertical variation in the plotted points corresponds to th ree different values of $V_{t, \mathrm{P}}$ used $(0.01,0.25$, and $0.5 \mathrm{~h})$ conditional on each value of $V_{\mathrm{ke}}$ used. To these points was fitted the linear quadratic curve shown: $\mathrm{Z}=(0.05053 \mathrm{~h}) V_{\mathrm{ke}}+\left(1.6608 \mathrm{~h}^{2}\right) V_{\mathrm{ke}}{ }^{2}$. For $V_{\mathrm{ke}}<0.04 \mathrm{~h}^{-1}$, the relative error of this fit using only the linear term $(0.05053 \mathrm{~h})$ is $<5 \%$. 
and where $V_{\mathrm{W}}$ was previously defined (after Equation 2). It was further assumed that variability in $V_{\mathrm{ke}}$ is lognormally distributed. Experimental data reported for 17 male and female human subjects indicates that $V_{\mathrm{ke}}$ has a CV of $\sim 0.60$ (Fisher et al., 1998). Based on the method of moments (Appendix 1; see discussion concerning $V_{\max }$ following Equation 8), it follows that $V_{\mathrm{e}}$ has an $\mathrm{AM}$ of 1 and that $V_{\mathrm{e}} \sim \mathrm{LN}(-0.152,0.551)$. Consequently, Equation 14 implies that $V_{\mathrm{ke}}<0.030 \mathrm{~h}^{-1}$ for virtually ( $>99 \%$ of) all modeled individuals at risk. Because $V_{t, \text { ing }}<0.5 \mathrm{~h}$ is assumed as described above, Approximation $13 \mathrm{~b}$ is accurate (to within $<2.5 \%$ ), and was thus used to evaluate Equation 11. These two equations, together with assumptions stated above, yield:

$$
\begin{aligned}
& D_{\mathrm{C}, \mathrm{P}}=\left(X_{\mathrm{C}, \mathrm{P}} \times 1 \mathrm{~d}\right) \frac{V_{\mathrm{fm}, \mathrm{P}}}{V_{\mathrm{fd}}}\left(\frac{0.6107 V_{\mathrm{W}}^{0.3}}{V_{\mathrm{e}}}+0.2074\right), \text { for } \mathrm{P}=\{\mathrm{ing}, \mathrm{der}\} \text {, and } \\
& D_{\mathrm{C}, \text { inh }}=\left(X_{\mathrm{C}, \text { inh }} \times 1 \mathrm{~d}\right) \frac{V_{\mathrm{fm}, \text { inh }}}{V_{\mathrm{fd}}}\left(\frac{7.878}{V_{\mathrm{e}}}+\frac{2.674}{V_{\mathrm{W}}^{0.3}}\right),
\end{aligned}
$$

in which no more than three significant digits are implied, and where: $X_{C, P}$ for pathways $\mathrm{P}=\{$ ing, inh, or der $\}$ were defined by Equations $1 \mathrm{a}-\mathrm{c}, V_{\mathrm{W}}$ was defined after Equation 2, $V_{\mathrm{fm}, \mathrm{P}}$ for pathways $\mathrm{P}$ were defined in Equations $9 \mathrm{a}-\mathrm{b}, V_{\mathrm{fd}}$ was defined after Equation 10, and $V_{\mathrm{e}}$ was defined after Equation 14. Note that $V_{\mathrm{fd}}$ and $V_{\mathrm{W}}$ are assumed to be correlated (as discussed prior to Equation 14), as are $V_{\text {alv }}$ and $V_{\text {inh }}$ as discussed above (in Section 2.3.3, after Equation 9b).

\subsection{TCE Dose-Response}

The following subsections discuss methods used to model dose-response for TCEinduced cancer and noncancer endpoints, and associated JUV. Sections 2.4.1 and 2.4.2 describe methods used for dose-response modeling under $\mathrm{MA}_{\mathrm{G}}$ and $\mathrm{MA}_{C}$, respectively. Section 2.4.3 then describes the method used to incorporate uncertainty concerning the mechanism of action for TCE-induced cancer into estimates of cancer risk as well as of corresponding aggregate (cancer and noncancer) risk.

\subsubsection{Dose-response assuming genotoxic mechanism(s)}

Under $\mathrm{MA}_{\mathrm{G}}$, linear-no-threshold extrapolation of TCE cancer risk is based on the assumption that TCE can increase cancer risk via one or more genotoxic mechanisms of 
action. These mechanisms involve DNA damage that is presumed to be linearly proportional to the biologically effective concentration of one or more of TCE's reactive metabolites, where potency is estimated for each bioassay in terms of a pharmacologically based equivalent effective dose-namely, the total amount of TCE metabolized per kg body weight per day (Bogen, 1988; USEPA, 1985, 1987b). Effective bioassay doses $D_{\mathrm{C}, \mathrm{P}}$ and corresponding positive, malignant (plus, where applicable, benign) tumor responses in mouse liver and rat kidney were obtained from information listed in Table 4 of Bogen (1988) concerning seven rodent bioassay data sets (Bell et al., 1978; Maltoni et al., 1986; NCI, 1976; NTP, 1990). The studies involved are summarized below in Table 1. For each data set, a cdf reflecting uncertainty (estimation-error) in estimated cancer potency (i.e., "slope factor", or risk per unit dose), here denoted $U_{\text {pot }}$ $\left(\mathrm{kg} \mathrm{d} \mathrm{mg}^{-1}\right.$ ), was calculated as described below (Section 2.6).

A subjective weighting scheme was then used to address uncertainty associated with lack of knowledge concerning which of the multiple positive animal bioassay results for TCE in rodent liver and kidney best predicts TCE cancer risk in humans, similar to an approach previously applied to characterize JUV in cancer risk posed by environmental exposure to chloroform (Bogen, 1995). To each species/sex-specific potency distribution obtained as described above, the corresponding relative weight indicated in Table 1 was applied to obtain a single weighted-average distribution reflecting uncertainty in tumor likelihood, conditional on effective dose. (This weighted average was obtained analytically, via calculations analogous to those indicated in Equation $5 \mathrm{~d}$.) The weights used assign equal likelihood (of reflecting true carcinogenic potency in humans) to bioassay data sets that differ: by sex within a given strain, by strain within a given species, and by species.

Animal-to-human extrapolation of toxicokinetically equivalent effective dose was done by using an appropriate PBPK model as described above, so no additional factor was employed in this regard in accordance with currently proposed policy (see Section 1.4). An uncertain factor $U_{\text {tdyn }}$ was used to account for interspecies toxicodynamic dynamic differences between rodents and humans (i.e., in increased likelihood of cancer per unit effective genotoxic dose). Analogous to toxicodynamic factors recommended recently by the USEPA for noncarcinogens and "nonlinear" 
Table 1. Bioassay data sets used to estimate potency of TCE as a genotoxic/linear liver or kidney carcinogen

\begin{tabular}{|c|c|c|c|c|c|c|c|c|}
\hline No. & Study ${ }^{a}$ & Species & Strain & Sex & Route $^{b}$ & $\begin{array}{c}\text { Tumor } \\
\text { type }^{b}\end{array}$ & $\begin{array}{l}\text { No. } \\
\text { dose } \\
\text { grps. }\end{array}$ & $\begin{array}{c}\text { Relative } \\
\text { study } \\
\text { weight }^{c}\end{array}$ \\
\hline 1 & NCI (1976) & mouse & $\mathrm{B} 6 \mathrm{C} 3 \mathrm{~F} 1$ & $\mathrm{M}$ & gav & $\mathrm{HCC}$ & 3 & 1 \\
\hline 2 & NCI (1976) & mouse & $\mathrm{B} 6 \mathrm{C} 3 \mathrm{~F} 1$ & F & gav & $\mathrm{HCC}$ & 3 & 1.5 \\
\hline 3 & NTP (1990) & mouse & B6C3F1 & M & gav & HCA & 2 & 1 \\
\hline 4 & NTP (1990) & mouse & B6C3F1 & $\mathrm{F}$ & gav & $\mathrm{HCA}$ & 2 & 1.5 \\
\hline 5 & NTP (1990) & rat & F344 & M & gav & RTCA & 3 & 12 \\
\hline 6 & Bell et al. (1978) & mouse & $\mathrm{B} 6 \mathrm{C} 3 \mathrm{~F} 1$ & $\mathrm{M}$ & inh & $\mathrm{HCA}$ & 3 & 1 \\
\hline 7 & Maltoni et al. (1986) & mouse & Swiss & $\mathrm{M}$ & inh & $\mathrm{MH}$ & 3 & 6 \\
\hline
\end{tabular}

'More detailed study-specific information appears in Table 4 of Bogen (1988).

${ }^{b}$ Lifetime bioassay exposure scenarios: gav = gavage $5 \mathrm{~d} / \mathrm{wk}$ in oil vehicle; inh $=$ inhalation $6 \mathrm{~h} / \mathrm{d}$, $5 \mathrm{~d} / \mathrm{wk}$. Tumor types: $\mathrm{HCC}=$ hepatocellular carcinomas; $\mathrm{HCA}=\mathrm{HCC}$ or hepatocellular adenomas; RTCA = renal tubule-cell carcinomas or adenomas; $\mathrm{MH}=$ malignant hepatomas.

${ }^{c}$ Assigned a priori relative study weight (see text).

carcinogens (USEPA, 1998), but using a probabilistic approach as previously proposed for noncancer endpoints (Carlson-Lynch et al., 1999; Slob and Pieters, 1998), it was assumed $U_{\mathrm{tdyn}}$ is lognormally distributed, has a GM of 1 (i.e., is as likely as not to exceed $1)$, and is unlikely $(\mathrm{p}<0.01)$ to exceed a value of 3 . A similar factor $V_{\mathrm{tdyn}}$ used to reflect intraspecies toxicodynamic variation was assumed to have an $A M$ of 1 and to be unlikely ( $p<0.01$ ) to exceed 10 . By the method of moments (Appendix 1 ), it thus was assumed that $U_{\mathrm{tdyn}} \sim \mathrm{LN}(0,1.60)$ and $V_{\mathrm{tdyn}} \sim \mathrm{LN}(0.700,2.33)$.

Combining the dose-response factors discussed above, and noting that $\overline{V_{\text {tdyn }}}=1$, increased risk $R_{G}$ under $M A_{G}$ was defined using a low-dose-linear multistage riskextrapolation model as

$$
\begin{aligned}
& R_{\mathrm{G}}=1-\exp \left\{-\left[U_{\mathrm{pot}} U_{\mathrm{tdyn}} V_{\mathrm{tdyn}}\left\langle\overline{D_{\mathrm{G}}} \sum_{\mathrm{P}=\text { (ing,inh,der }\}} f_{\mathrm{G}, \mathrm{P}} B_{\mathrm{G}, \mathrm{P}}\right]\right\}, \quad\right. \text { with } \\
& \overline{R_{\mathrm{G}}} \approx 1-\exp \left(-U_{\mathrm{pot}} U_{\mathrm{tdyn}}\left\langle\overline{D_{\mathrm{G}}}\right\rangle \overline{B_{\mathrm{G}}}\right), \quad \text { and } \\
& \left\langle R_{\mathrm{G}}\right\rangle \approx 1-\exp \left\{-\left[\left\langle U_{\mathrm{pot}}\right\rangle\left\langle U_{\mathrm{tdyn}}\right\rangle V_{\mathrm{tdyn}}\left\langle\overline{D_{\mathrm{G}}}\right\rangle \sum_{\mathrm{P}=(\text { ing, inh, der })} f_{\mathrm{G}, \mathrm{G}}\left\langle B_{\mathrm{G}, \mathrm{P}}\right\rangle\right]\right\},
\end{aligned}
$$


in which $U_{\mathrm{pot}} U_{\mathrm{tdyn}}$ and $V_{\mathrm{tdyn}}$ were defined above in this subsection, and the remaining variates were defined in/after Equations $5 \mathrm{a}-\mathrm{b}$ with reference to Equation 6 . In Approximation $16 \mathrm{~b}, \overline{B_{\mathrm{G}}}=\overline{B_{\mathrm{G}, \mathrm{P}}}$ because (conditional on Equations 1a-c, on Equation 6 , and on all heterogeneous variates involved in $\left.B_{C, P}\right)$, uncertainty in $B_{C, P}$ is due entirely to uncertainty in the variates $E D$ and $C_{\mathrm{w}}$ (defined after Equations $4 \mathrm{~b}$ and $4 \mathrm{c}$, respectively) that are both independent of pathway $P$. Note that the $\left\langle B_{\mathrm{G}, \mathrm{P}}\right\rangle$ variates in Approximation $16 \mathrm{c}$ are correlated (see Section 2.6). Approximations $16 \mathrm{~b}-\mathrm{c}$ are $1^{\text {st }}$-order approximations (see Bogen and Spear, 1987). However, for extrapolation of risks $<10^{-2}$ the functions involved are effectively linear, so the approximations entail only negligible loss of accuracy.

\subsubsection{Dose-response assuming cytotoxic mechanism(s)}

The ability of TCE to induce cancer under $\mathrm{MA}_{C}$ was assumed to arise from TCAinduced cytotoxicity/mitogenicity indicated by increased formation of thiobarbituricacid-reactive substances (TBARS), as previously suggested (Bogen and Gold, 1997). Absent better data, increased TBARS elevation above background was modeled using data on male B6C3F1 mice administered a single gavage dose of $0,100,300,1000$, or $2000 \mathrm{mg}$ TCA per $\mathrm{kg}$ body weight in buffered water, and corresponding measured peak TCA concentrations in plasma, $\operatorname{Max}\left(C_{\mathrm{TCA}}\right)$ (Larson and Bull, 1992a). Multiple independent interactions are likely to be involved in TCA-induced oxidative-stress. Consequently (Aitchison and Brown, 1957), dose-response under $\mathrm{MA}_{C}$ could reasonably be modeled using a two-parameter LN function

$$
Y\left(A_{C}\right)-Y_{0}=100 \Phi\left(\frac{\log _{10}\left(A_{C}\right)-\mu}{\sigma}\right), \quad \text { in which }
$$

$A_{\mathrm{C}}=$ administered acute TCA dose (mg TCA per $\mathrm{kg}$ body weight);

$Y\left(A_{\mathrm{C}}\right)=$ TBARS level ( $\mathrm{nmol}$ malondialdehyde equiv. per g liver) induced by $A_{\dot{C}}$;

$Y_{0}=Y(0)=$ background TBARS level ( $\mathrm{nmol}$ malondialdehyde equiv. per $\mathrm{g}$ liver);

$\Phi(z)=$ cumulative probability distribution function (cdf) of a standard normal (Gaussian) random variate $Z$, equal to $\operatorname{Prob}(Z \leq z)$;

$\mu=$ location parameter $=\log _{10} \mathrm{GM}$ (unitless); and

$\sigma=$ shape parameter $=\log _{10} \mathrm{GSD}$ (unitless); 
to the mouse TBARS-vs.- $A_{\mathrm{C}}$ data (see Section 2.6), where the unit of $Y\left(A_{\mathrm{C}}\right)$ is henceforth suppressed for convenience. The arbitrary constant (100) in the two-parameter model (Equation 16) was used because a three-parameter LN model fit to these data did not yield plausibly unique parameter estimates.

Raw $Y\left(A_{\mathrm{C}}\right)$-vs. $-A_{\mathrm{C}}$ data ([4 measures] $\times[4$ noncontrol dose levels]) summarized by Larson and Bull (1992a) were assumed to be approximately normally distributed, with a reported arithmetic mean (AM) and standard deviation (SD) for $Y_{0}$ of 40.0 and $4.0 \mathrm{nmol}$ malondialdehyde equiv. per $g$ liver, respectively. Error in this AM was modeled as $t$ distributed with 3 degrees of freedom. TBARS elevations above the corresponding 2tail upper $95 \%$ confidence limit on $Y_{0}$ were assumed to be biologically significant in the sense of being plausibly related to TCA-induced cytotoxicity. This upper bound on $Y_{0}$ shall be denoted $Y_{\text {sigr }}$ and the mouse data indicate that $Y_{\text {sig }}=49.4 \mathrm{nmol}$ malondialdehyde equiv. per g liver. Estimates $\{\hat{\mu}, \hat{\sigma}\}$ of the $\operatorname{LN}$ parameters $\{\mu, \sigma\}$, as well as their corresponding estimated SDs $\left\{s_{\hat{\mu}}, s_{\hat{\sigma}}\right\}$ and product-moment correlation $(r)$, were obtained by fitting the LN-model (Equation 17) to the mouse TBARS data, as described below (Section 2.6).

Parameter-estimation error pertaining to the likelihood that any particular acute administered dose $A_{\mathrm{C}}$ would induce a significantly elevated (i.e., presumptively cytotoxic) response was characterized by simulating uncertainty in parameters $\mu$ and $\sigma$. This was done by modeling these parameters as the corresponding variates $\mu=\hat{\mu}+s_{\hat{\mu}} U_{\mathrm{t} 1}$, and $\sigma=\hat{\sigma}+s_{\hat{\sigma}} U_{\mathrm{t} 2}$, where $U_{\mathrm{t} 1}$ and $U_{\mathrm{t} 2}$ are correlated uncertain variates each distributed as Student's T with $[(4 \times 4)-2]=14$ degrees of freedom, having a correlation coefficient equal to $r$. At the lowest $A_{\mathrm{C}}$ level used $\left(100 \mathrm{mg} \mathrm{kg}^{-1}\right)$ in B6C3F1 mice, it was found that effective dose, expressed as the corresponding maximum plasma TCA concentration $\left(D_{\mathrm{Ca}}\right)$, was $D_{\mathrm{Ca}}=\operatorname{Max}\left(C_{\mathrm{TCA}}\right)=130 \mathrm{mg} \mathrm{L}^{-1}$ (Larson and Bull, 1992a). For convenience, and absent data at lower $D_{\mathrm{Ca}}$ levels, it was assumed that the ratio $D_{\mathrm{Ca}} / A_{\mathrm{C}}$ is a constant independent of $A_{\mathrm{C}}$, equal to $\left[\left(130 \mathrm{mg} \mathrm{L}^{-1}\right) /\left(100 \mathrm{mg} \mathrm{kg}^{-1}\right)\right]=$ $1.3 \mathrm{~kg} \mathrm{~L}^{-1}$. Critical effective dose conditional on $\{\mu, \sigma\}$ was therefore modeled as 
$D_{\mathrm{Ca}}=\left(1.3 \mathrm{mg} \mathrm{L}^{-1}\right) 10^{\mu+\sigma \Phi^{-1}\left[\left(Y_{\mathrm{sig}}-Y_{0}\right) / 100\right]}$

$=\left(1.3 \mathrm{mg} \mathrm{L}^{-1}\right) 10^{\left(\hat{\mu}+s_{\hat{\mu}} U_{t 1}\right)+\left(\hat{\sigma}+s_{\hat{\sigma}} U_{t 2}\right) \Phi^{-1}\left[\left(Y_{\mathrm{sig}}-Y_{0}\right) / 100\right]} \sim F_{\mathrm{C}}\left(D_{\mathrm{Ca}}\right)$, where

$D_{\mathrm{Ca}} \quad=$ acute effective TCA dose (mg TCA per L plasma);

$Y_{\text {sig }} \quad=$ significantly elevated value of $Y\left(A_{\mathrm{C}}\right)$ above $Y_{0}$ (defined above);

$U_{\mathrm{ti}}=($ for $i=1,2)$ correlated errors distributed as Student's $\mathrm{T}$ cdfs with $\mathrm{df}=14$; and

$F_{\mathrm{C}}\left(D_{\mathrm{Ca}}\right)=$ cdf of $D_{\mathrm{Ca}}$ specifying the modeled likelihood of significant TBARS elevation conditional on effective dose $D_{\mathrm{Ca}}$;

where $Y_{0}, \hat{\mu}, s_{\hat{\mu}}, \hat{\sigma}$, and $s_{\hat{\sigma}}$, as well as the $t$-distributed variates $U_{\mathrm{t} 1}$ and $U_{\mathrm{t} 2}$, were all defined above, and where " $\sim$ " means "is distributed as". Absent dose-response data on TCA-induced lipoperoxidation or cytotoxicity in rat kidney, it was assumed that $F_{\mathrm{C}}\left(D_{\mathrm{Ca}}\right)$ also applies to rat kidney. This assumption is probably conservative, because relation between $A_{\mathrm{C}}$ and $D_{\mathrm{Ca}}=\operatorname{Max}\left(C_{\mathrm{TCA}}\right)$ was observed to be similar in rats vs. mice, whereas TCA is less effective at inducing TBARS elevation in rats vs. mice (Larson and Bull, 1992a).

Detailed dose-response information relating chronically or subchronically administered TCA and induced TBARS or cytotoxicity are still unavailable. Therefore, extrapolation of $D_{\mathrm{Ca}}$ to equivalent subchronic effective TCA dose, and extrapolation of subchronic to chronic effective TCA dose (where the latter is denoted $D_{C}$, and is defined by Equations $5 \mathrm{a}, 11$, and 15a-b), was accomplished using the two uncertainty factors, $U_{\text {acute }}$ and $U_{\text {subchron }}$ respectively. As previously suggested (Slob and Pieters, 1998), these factors were assumed to be lognormally distributed. Based on the observation that ratio of lowest observed effect levels for TCE-induced lethality in B6C3F1 mice is between 2 and 3 (NCI, 1976), it was assumed that $U_{\text {acute }}$ has a $\mathrm{GM}$ of $\mathrm{GM}_{\text {acute }}=3$ and is unlikely ( $p<0.01$ ) to be greater than 6 . The factor $U_{\text {subchron }}$ was assumed by default (see Slob and Pieters, 1998) to have a GM of $\mathrm{GM}_{\text {subchron }}=\sim 2$ and to be unlikely $(\mathrm{p}<0.01)$ to exceed 10 . Because many repeated daily exposures to TCE and/or its metabolite TCA are expected to be always more (never less) toxic than fewer exposures, it was assumed that a combined uncertainty factor $\left(U_{\text {chron }}\right)$ extrapolates effective dose from a chronic to a toxicologically equivalent acute (bioassay) exposure condition as follows: 


$$
\begin{aligned}
& D_{\mathrm{Ca}}=\left(1+U_{\text {chron }}\right) D_{\mathrm{C}}, \text { where } \\
& U_{\text {chron }}=U_{\text {acute }} \times U_{\text {subchron }}\left(1-\left(\mathrm{GM}_{\text {acute }} \mathrm{GM}_{\text {subchron }}\right)^{-1}\right) .
\end{aligned}
$$

That is, the combined LN factor $U_{\text {chron }}$ was assumed to have a GSD equal to that of $U_{\text {acute }} \times U_{\text {subchron }}$ and a GM equal to one less than that of $U_{\text {acute }} \times U_{\text {subchron. }}$. By the method of moments (Appendix 1), it was thus assumed that $U_{\text {chron }} \sim \mathrm{LN}(\ln 5, \ln 2.12)$.

Animal-to-human extrapolation of toxicokinetically equivalent effective dose was done by using an appropriate PBPK model as described above, so no additional factor was employed in this regard in accordance with currently proposed policy (see Section 1.4). An uncertain factor $U_{\mathrm{tdyn}}$ was used to account for interspecies toxicodynamic dynamic differences between rodents and humans, and a similar factor $V_{\text {tdyn }}$ to reflect intraspecies toxicodynamic variation, where $U_{\text {tdyn }}$ and $V_{\text {tdyn }}$ were defined above (in Section 2.4.1, prior to Equation 16).

Combining the dose-response factors discussed above, , and again noting that $\overline{V_{\text {tdyn }}}=1$, increased risk $R_{\mathrm{C}}$ under $\mathrm{MA}_{\mathrm{C}}$ was modeled as

$$
\begin{aligned}
& R_{\mathrm{C}}=F_{\mathrm{C}}\left(U_{\mathrm{tdyn}} V_{\mathrm{tdyn}}\left(1+U_{\text {chron }}\right)\left\langle\overline{D_{\mathrm{C}}}\right\rangle_{\mathrm{P}=\{\text { (ing,inh,der\} }} f_{\mathrm{C}, \mathrm{P}} B_{\mathrm{C}, \mathrm{P}}\right), \quad \text { with } \\
& \overline{R_{\mathrm{C} 1}}=F_{\mathrm{C}}\left(U_{\mathrm{tdyn}}\left(1+U_{\text {chron }}\right)\left\langle\overline{D_{\mathrm{C}}}\right\rangle \overline{B_{\mathrm{C}}}\right), \quad \text { and } \\
& \left\langle R_{\mathrm{C}}\right\rangle_{1}=F_{\mathrm{C}}\left(\left\langle U_{\mathrm{tdyn}}\right\rangle V_{\mathrm{tdyn}}\left(1+\left\langle U_{\text {chron }}\right\rangle\right)\left\langle\overline{D_{\mathrm{C}}}\right\rangle \sum_{\mathrm{P}=\{\text { ing, inh, der }\}} f_{\mathrm{C}, \mathrm{C}, \mathrm{P}}\left\langle B_{\mathrm{P}}\right\rangle\right),
\end{aligned}
$$

in which $U_{\text {chron }}, U_{\mathrm{tdyn}}$ and $V_{\mathrm{tdyn}}$ were defined above in this subsection; the remaining variates were defined in/after Equations 5a-c with reference to Equations 11 and 15a-b. In Approximation 20b, $\overline{B_{\mathrm{C}}}=\overline{B_{\mathrm{C}, \mathrm{P}}}$ because (conditional on Equations 1a-c and 15a$\mathrm{b}$, and on all heterogeneous variates involved in $\left.B_{C, P}\right)$ uncertainty in $B_{C, P}$ is due entirely to uncertainty in the variate $C_{\mathrm{w}}$ (defined after Equation $4 \mathrm{c}$ ), which in turn is independent of pathway P. Note that the $\left\langle B_{\mathrm{C}, \mathrm{P}}\right\rangle$ variates in Approximation 20c are correlated (see Section 2.6).

The subscripts " 1 " on the left side of Equations $20 \mathrm{~b}-\mathrm{c}$ each denote a $1^{\text {st }}$-order approximation, as was the case in Approximations 16b-c. However, Equations 20b-c 
are expected to underestimate risk, because (in contrast to the exponentiated polynomial in Approximations $16 \mathrm{~b}-\mathrm{c}$ ) $F_{\mathrm{C}}$ is a substantially nonlinear increasing function of effective dose (see Bogen and Spear, 1987). In the risk range from $10^{-10}$ to $10^{-2}$ relevant to this analysis, $\log \left(F_{\mathrm{C}}\left(D_{\mathrm{Ca}}\right)\right)$ turns out to be well modeled by a linear-quadratic function of effective dose $D_{\mathrm{Ca}}$, as explained below (Sections 2.6 and 3.2). Therefore, more accurate, corresponding $2^{\text {nd }}$-order approximations were used to evaluate $R_{\mathrm{C}}$ expectations, which were calculated as follows. For any risk $R$ that is a function of a vector of $n$ uncertain and/or heterogeneous variates $X=\left\{X_{1}, X_{2}, \ldots, X_{n}\right\}$, the $2^{\text {nd }}$-order approximation of the expectation $E R(X)$-with respect only to variability, or only to uncertainty $\longrightarrow$ of $R$, is given by (cf. Bogen and Spear, 1987):

$$
\mathrm{E}_{2} R(\mathrm{X})=\mathrm{E}_{1} R(\mathrm{X})+\frac{1}{2} \sum_{i=1}^{k} \frac{\partial^{2} R(\mathrm{X})}{\partial \mathrm{X}_{i}^{2}} \sigma_{\mathrm{X}_{i}}^{2}
$$

in which subscripts " 1 " and "2" on E denote the order of approximate expectation, $0 \leq k \leq n, k=$ the size of the subset of $\mathbf{X}$ comprising all members of $\mathbf{X}$ that are variates of the type corresponding to expectation $\mathrm{E}$ (e.g., comprising all uncertain variates among $\mathrm{X}$ if $\mathrm{E}$ represents expectation with respect only to uncertainty in $R(\mathrm{X})), \sigma^{2}$ denotes the variance of the $\sigma$-subscripted variate with respect to its uncertainty or to its variability (again corresponding to how $\mathrm{E}$ is defined), and the second derivatives are all evaluated conditional on $\mathbf{X}=\mathrm{EX}$. Using Equation 21, second-order approximations were obtained to estimate conditional risks under $\mathrm{MA}_{\mathrm{C}}$ corresponding to Equations 20b-c (see Section 2.6).

\subsubsection{Model Uncertainty}

In view of the plausibility of both $\mathrm{MA}_{\mathrm{G}}$ and $\mathrm{MA}_{\mathrm{C}}$ (see Section 1.3), uncertainty in the mechanism(s) of carcinogenic action for TCE was treated quantitatively, based on the "dichotomous" mechanistic assumption ( $\mathrm{MA}_{\mathrm{GuC}}$ ) involving both $\mathrm{MA}_{\mathrm{G}}$ and $\mathrm{MA}_{\mathrm{C}}$ discussed above (Section 2.3). The alternative corresponding "composite" assumption $\left(\mathrm{MA}_{\mathrm{G} C \mathrm{C}}\right.$ ), which also involves both $\mathrm{MA}_{\mathrm{G}}$ and $\mathrm{MA}_{\mathrm{C}}$ (as discussed in Section 2.3), is more difficult to implement quantitatively than $\mathrm{MA}_{\mathrm{GuC}}$. $\mathrm{MA}_{\mathrm{G} \cap \mathrm{C}}$ is more difficult to implement because it requires a complete model structure accounting for possible but unknown interactions between the different mechanisms considered. In contrast, $\mathrm{MA}_{\mathrm{GuC}}$ may be implemented simply by assigning the component assumptions $\left(\mathrm{MA}_{\mathrm{G}}\right.$ and $\left.M \mathrm{~A}_{C}\right)$ 
corresponding, complementary a priori probabilities, and using the combination of these probabilities to reflect the (quantitatively equivalent) possibility that both $\mathrm{MA}_{G}$ and $\mathrm{MA}_{\mathrm{C}}$ are true but to unknown degrees. $\mathrm{MA}_{\mathrm{GuC}}$ was therefore adopted using subjective probabilities $U_{\mathrm{G}}$ and $U_{\mathrm{C}}=\left(1-U_{\mathrm{G}}\right)$ to reflect the corresponding likelihoods that $\mathrm{MA}_{\mathrm{G}}$ and $\mathrm{MA}_{\mathrm{C}}$, respectively, reflect the "true" mechanism of TCE-induced carcinogenic action. Consistent with our considered opinion that $\mathrm{MA}_{\mathrm{C}}$ is at least as likely as not to explain observed TCE-induced cancer in rodent bioassays (see also Bogen and Gold, 1997), $U_{G}$ was modeled as uniformly distributed between 0 and 0.5 . Therefore, using de Morgan's rule (see Appendix I in NRC, 1994), increased aggregate risk $R$ of incurring either genotoxicity-induced cancer, or cytotoxicity (which, in the case of TCE, may indirectly increase cancer risk), due to TCE exposure at Site LF-13 was modeled as

$$
R=1-\left(1-U_{\mathrm{G}} R_{\mathrm{G}}\right)\left(1-R_{\mathrm{C}}\right)
$$

in which $U_{\mathrm{G}}$ was just defined; $R_{\mathrm{G}}$ and $R_{\mathrm{C}}$ were defined by Equations $16 \mathrm{a}$ and $20 \mathrm{a}$, respectively; and correlations between $R_{\mathrm{G}}$ and $R_{\mathrm{C}}$ were incorporated (see Section 2.6).

\subsection{Risk Characterization}

Increased health risk and related JUV associated with residential exposure to TCE from ground water at Site LF-13 on Beale Air Force Base in California was characterized quantitatively using notation similar to those used in the Phase-1 report (Daniels et al., 2000). Specifically, increased individual risk $R$ defined by Equation 22 was evaluated using established methods (Bogen, 1995; Bogen and Spear, 1987; NRC, 1994) to obtain mean and upper-bound values of the conditional expectations $\bar{R}$ and $\langle R\rangle$, where the cdf for $\bar{R}$ represents uncertainty in risk to a (hypothetical) person at a population-average level of risk relative to others, and the cdf of $\langle R\rangle$ represents interindividual variability in the expected values of risk predicted for different people. A subscript $p(0 \leq p \leq 1)$ on either of these conditional expectations is used to denote a $100 p$ th percentile at which the corresponding cdf is evaluated, while $R_{u, v}(0 \leq u \leq 1$, $0 \leq v \leq 1$ ) is used to denote joint $100 u$ th-uncertainty and $100 v$ th-variability percentiles with respect to JUV in $R$. Estimates of $R_{u, v}$ were obtained jointly conditional on one of three upper bounds $u(0.50,0.95$ or 0.99$)$ with respect to aggregate uncertainty, and on one upper bound $(v=0.99)$ with respect to aggregate variability. These $R_{u, v}$ estimators 
characterize median and upper uncertainty bounds on risk to a person who is relatively highly at risk compared to others at risk.

The JUV-explicit estimators of individual risk obtained (involving $\bar{R},\langle R\rangle$, and $R_{u, v}$ ) were compared to traditional point-estimates of risk $\hat{R}_{\mathrm{RME}}$ and $\hat{R}_{\text {High }}$ taken from Daniels et al. (2000). The $\hat{R}_{\mathrm{RME}}$ estimate was calculated entirely analytically, using regulatory default values for all input variates where available; where default values were not available, expected values were used for all uncertain variates, and upper/unlikely bounds (e.g., $95^{\text {th }}$ percentile values) were used for all heterogeneous variates. The $\hat{R}_{\text {High }}$ estimate was similarly calculated using only upper/unlikely bounds for all input variates.

Also of potential interest to stakeholders and decision makers are corresponding estimates of population risk, that is, of the uncertain total number $N$ of additional cases of TCE-induced cancer or noncancer associated with population exposure to risk $R$. For an exposed population of total size $n, N$ has an expected value of $\langle N\rangle=n\langle\bar{R}\rangle$, and the probability $p_{0}$ that there will be zero additional cases (and consequently zero health benefit from efforts to reduce $R$ ) is well approximated by the integral of the conditional Poisson likelihood function

$$
p_{0} \approx \int_{0}^{1} e^{-n \bar{R}} f_{\bar{R}}(r) \mathrm{d} r
$$

in which $f_{\bar{R}}(r)$ is probability density function of the uncertain conditional expectation $\bar{R}$ referred to above (in reference to $R$ defined by Equation 22 ), and the compoundPoisson rate $(n \bar{R})$ incorporates this same conditional expectation (see Bogen and Spear, 1987; NRC, 1994; Bogen, 1995).

\subsection{Data Analysis and Computation}

Uncertain cancer potency $U_{\text {pot }}$ for each animal-bioassay data set was calculated using a computationally efficient, non-asymptotic, analytic-bootstrap method previously described (Bogen, 1994). Briefly, potency was estimated for each bioassay data set using least-squares polynomial-regression fits of a polynomial in LTWA effective bioassay dose $D_{\mathrm{G}}$ to 500 simulated values of $-\ln \left[1-P\left(D_{\mathrm{G}}\right)\right]$, based on observed 
tumor-occurrence rates $P\left(D_{\mathrm{G}}\right)$ at each level of $D_{\mathrm{G}}$ used, and under the constraint that all fitted polynomial coefficients are nonnegative. The polynomial degree was specified in the usual way, as previously described (Anderson et al., 1983). Uncertainty in $U_{\text {pot }}$ reflected by each data set was then modeled as the empirical distribution corresponding to the 500 resulting fitted values of potency, defined as the linear coefficient in dose.

Estimated parameter and asymptotic SD values were obtained for a lognormal model (Equation 17) fit to mouse TBARS-vs.- $A_{C}$ data by Levenberg-Marquardt minimization of $X^{2}$, the sum of weighted squared deviations of observed from predicted values; corresponding goodness-of-fit was assessed as $\operatorname{Prob}\left(\mathrm{X}^{2}>\chi^{2}\right)$ for $\chi^{2}$ distributed as chi-square with degrees of freedom (df) equal to the number of data points minus the number of estimated parameters (Press et al., 1992). The weight used for each $A_{\mathrm{C}}$ level was the corresponding value $s^{-2}$, where $s=$ the SD of raw TBARS measures calculated from the SD of the corresponding mean TBARS value reported by (Larson and Bull, 1992a).

Equation 18 was evaluated numerically in order to calculate the risk of cytotoxic response, $F_{\mathrm{C}}\left(D_{\mathrm{Ca}}\right)$, as a function of $D_{\mathrm{Ca}}$ over the interval $0 \leq F_{\mathrm{C}}\left(D_{\mathrm{Ca}}\right) \leq 1$. This numerical evaluation made use of the fact that the exponentiated expression in Equation 18 involving $U_{\mathrm{t} 1}$ and $U_{\mathrm{t} 2}$ represents a linear function of correlated $t$-distributed variates, namely: $\quad \mathrm{w}_{0}+\mathrm{w}_{1} U_{\mathrm{t} 1}+\mathrm{w}_{2} U_{\mathrm{t} 2} \quad$ where $\quad \mathrm{w}_{0}=\hat{\mu}+\hat{\sigma}\left\{\Phi^{-1}\left[\left(Y_{\mathrm{sig}}-Y_{0}\right) / 100\right]\right\}, \quad \mathrm{w}_{1}=s_{\hat{\mu}}$ $\mathrm{w}_{2}=s_{\hat{\sigma}}\left\{\Phi^{-1}\left[\left(Y_{\mathrm{sig}}-Y_{0}\right) / 100\right]\right\}$, and a correlation $r$ is assumed between $U_{\mathrm{t} 1}$ and $U_{\mathrm{t} 2}$. Exact and corresponding approximate expressions are available for the distribution of linear functions of independent $t$-distributed variates (Ruben, 1960; Patil, 1965; Ghosh, 1975; Walker and Saw, 1978; Chaubey and Mudholkar, 1982; Ojo, 1988; Singh, 1990), but not for correlated $t$-distributed variates. The distribution of any linear function of correlated $t$-distributed variates is derived in Appendix 2. In particular, the weighted sum of two $t$-distributed variates $U_{\mathrm{t} 1}$ and $U_{\mathrm{t} 2}$ with correlation $r$ and with degrees of freedom $f_{1}$ and $f_{2}$, respectively, is given by:

$$
\begin{aligned}
& F(v)=\int_{0}^{1} \operatorname{Prob}\left(T_{f_{1}+f_{2}} \leq v \phi(x)\right)\left[\mathrm{d} \beta\left(x_{1} f_{1} / 2, f_{2} / 2\right) / \mathrm{d} x\right] \mathrm{d} x, \text { where } \\
& \phi^{2}(x)=\frac{\left(f_{1}+f_{2}\right) x(1-x)}{f_{1}(1-x) w_{1}^{2}+f_{2} x w_{2}^{2}+2 r w_{1} w_{2} \sqrt{f_{1} f_{2} x(1-x)}},
\end{aligned}
$$


and where in Equation $24 \mathrm{a}, T_{f_{1}+f_{2}}$ denotes a Student's $t$-distributed variate with $f_{1}+f_{2}$ degrees of freedom, and $\beta$ denotes the incomplete beta-function ratio corresponding to the specified arguments (see Abbreviations and Notation). To approximate $F_{\mathrm{C}}\left(D_{\mathrm{Ca}}\right)$ over relevant risk range $\left(\sim 10^{-10}\right.$ to $\left.\sim 10^{-2}\right)$, the linear-quadratic function $\log _{10}$ (risk) $=a+b x+c x^{2}$ was fit by unweighted least squares to points on $F_{\mathrm{C}}\left(D_{\mathrm{Ca}}\right)$ calculated within this risk range, where $x=\log _{10}\left(D_{\mathrm{Ca}}\right)$. The following second-order approximation was then obtained using Equation 21 (in Section 2.4) to estimate corresponding conditional risks under $\mathrm{MA}_{\mathrm{C}}$ (see Appendix 3.H, pp. $\mathrm{H}-9$ to $\mathrm{H}-10$ ):

$$
\mathrm{E} R_{\mathrm{C}} \approx \mathrm{E}_{1} R_{\mathrm{C}}+\frac{10^{a}}{2} \Pi^{b+c L}(\lambda+b(b-1)+2 c L[2(b+c L)-1])\left(\gamma_{X_{1}}^{2}+\gamma_{X_{2}}^{2}\right) .
$$

Equations $20 \mathrm{~b}$ and $21 \mathrm{imply}$, in particular, that $\overline{R_{\mathrm{C}}}$ is estimated by Equation 25 in which: E denotes expectation with respect to variability only; $a, b$, and $c$ are the linear-quadratic coefficients defined above; $\Pi=K U V_{1} V_{2} ; K=\left\langle\overline{D_{\mathrm{C}}}\right\rangle ; U=U_{\text {tdyn }}\left(1+U_{\text {chron }}\right) \overline{B_{\mathrm{C}}} ; V_{1}=V_{\text {tdyn }}$; $V_{2}=\left\langle B_{\mathrm{C}}\right\rangle ; \lambda=2 c / \ln 10$ (where $\ln$ denotes natural logarithm); $L=\log _{10} \Pi ; \gamma_{\mathrm{X}}=\left(\sigma_{\mathrm{X}} / \mathrm{EX}\right)=$ the coefficient of variation of the subscripted variate $X ; X_{1}=V_{1}$; and $X_{2}=V_{2}$. Likewise, Equations $20 \mathrm{c}$ and $21 \mathrm{imply}$ that $\left\langle R_{\mathrm{C}}\right\rangle$ is estimated by Equation 25 in which: E denotes expectation with respect to uncertainty only; $\Pi=\left[K V U_{1}\left(\sum_{i} U_{i}\right)\right]$ for $i=\{$ ing, inh, der $\}$; $V=V_{\text {tdyn }}\left\langle B_{\mathrm{C}}\right\rangle ; U_{1}=U_{\text {tdyn }}\left(1+U_{\text {chron }}\right) ; U_{i}=f_{\mathrm{C}, i} \overline{B_{\mathrm{C}}} ; L=\log _{10} \Pi ; K, \lambda$ and $\gamma$ were defined above; $X_{1}=U_{1}$; and $X_{2}=\sum_{i} U_{i}$ for $i=\{$ ing, inh, der $\}$, noting that $\mathrm{EX}_{1}=\mathrm{EX}_{2}=1$ and that $\sigma_{X_{2}}^{2}=\left(\sum_{i} f_{C, i}^{2}\right) \sigma_{\overline{B_{C}}}^{2}$. Equation 24 was evaluated taking all moments with respect to the same distributed characteristic (i.e., uncertainty or variability, corresponding to $E$ as defined above), using estimates for $a, b$, and $c$, and for indicated variate moments, that are implied by assumptions used (see Appendix 1) or are reported in Results (Section 3).

Monte-Carlo methods were used to generate sample values for each of (say, $k$ ) distributed variates involved in a given calculation. Specifically, systematic Latinhypercube sampling was used to simulate $n_{\text {sam }}$ samples of each required set of $k$ variates, where $k$ was determined by the equation(s) being evaluated, and a method 
(Iman and Conover, 1982) was used to obtain rank-correlated sample vectors, each with a rank-correlation matrix $M_{\mathrm{i}}(i=1, \ldots, k)$ not significantly different $\left(p_{\text {adj }}>0.05\right)$ from a specified target matrix $T$, which by default was a $k \times k$ identity matrix modified to reflect correlations specified below. A value of $n_{\text {sam }}=2000$ was used unless otherwise specified. The $k$ differences between $M_{\mathrm{i}}$ and $T$ were each assessed using an asymptotic chi-square test (Jennrich, 1970), and the p-value from each test was adjusted (to $p_{\text {adj, }}$ to account for $k$ independent tests) using Hommel's Bonferroni-type procedure (Wright, 1992). Typically, $\operatorname{Min}\left(p_{\text {adj }}\right)>0.95$; occasional sample vectors not satisfying $p_{\text {adj }}>0.01$ were rejected. Each simulation was repeated $n_{\text {sim }}$ times, a grand AM and its CV (denoted CVM, where CVM $=\mathrm{CV}\left[n_{\text {sim }}\right]^{-0.5}$ ) from the $n_{\text {sim }}$ cdf-specific AMs, and the AM and CVM were calculated for each $i$ th set of $n_{\text {sim }}$ cdf-specific order statistics (i.e., cdfabscissa values), where $i=1, \ldots, n_{\text {sam }}$. The calculated CVM values reflect simulation quality by indicating the relative size of Monte-Carlo sampling error produced for estimators of interest conditional on the values of $n_{\text {sam }}$ and $n_{\text {sim }}$ used.

Target rank-correlation values or matrices were estimated for all sets of correlated variates noted or implied above, namely, the sets: $\left\{V_{\mathrm{fm}, \text { inh }} V_{\mathrm{alv}}\right\},\left\{V_{\mathrm{fd}}, V_{\mathrm{W}}\right\}$, $\left\{\left\langle B_{\mathrm{G}, \text { ing }}\right\rangle,\left\langle B_{\mathrm{C}, \text { ing }}\right\rangle,\left\langle B_{\mathrm{G}, \text { inh }}\right\rangle,\left\langle B_{\mathrm{C}, \text { inh }}\right\rangle,\left\langle B_{\mathrm{G}, \text { der }}\right\rangle,\left\langle B_{\mathrm{G}, \text { der }}\right\rangle\right\},\left\{\overline{B_{\mathrm{G}}}, \overline{B_{\mathrm{C}}}\right\}$, and $\left\{U_{\mathrm{t} 1}, U_{\mathrm{t} 2}\right\}$ (see Results, Sections 3.1 and 3.2). These correlations were used, respectively, to evaluate: $D_{\mathrm{G}, \text { inh }}$ and $D_{\mathrm{C}, \text { inh }}$ in Equations 6 and 15b; $D_{\mathrm{C}, \mathrm{P}}$ in Equations 15a-b; $\left\langle B_{\mathrm{MA}, \mathrm{P}}\right\rangle$ in Equations $16 \mathrm{c}$ and 20c; $\overline{B_{\mathrm{MA}}}$ in Equations $16 \mathrm{~b}$ and $20 \mathrm{~b}$, and $F_{\mathrm{C}}\left(D_{\mathrm{Ca}}\right)$ in Equation 18. In calculations to estimate correlations involving $\left\langle B_{\mathrm{MA}, \mathrm{P}}\right\rangle$, values of $n_{\text {sim }}=500$ and $n_{\text {sam }}=50$ were used.

Correlations involving all the variate sets listed above were used in nested (i.e., two-dimensional) Monte-Carlo evaluations of Equation 22 (which, in this case, refers to Equations $16 \mathrm{a}$ and 20a) that were performed to estimate $R_{u, v}$. For these nested calculations, values of $n_{\text {sim }}=100$ and $n_{\text {sam }}=999$ were used, with $n_{\text {sam }}$ used to simulate all uncertain variates, and then used again to simulate all heterogeneous variates conditional on each of the $n_{\text {sam }}$ simulated sets of uncertain variates. Because $\left\langle B_{\mathrm{MA}}\right\rangle \approx\left\langle B_{\mathrm{MA}, \mathrm{P}}\right\rangle$ and $\overline{B_{\mathrm{MA}}} \approx \overline{B_{\mathrm{MA}, \mathrm{P}}}$ for all pathways $\mathrm{P}$ (see Results, Section 3.1), $\left\langle B_{\mathrm{MA}}\right\rangle$ and $\overline{B_{\mathrm{MA}}}$ were used to evaluate Equations $16 \mathrm{~b}$ and $20 \mathrm{~d}$, rather than $\left\langle B_{\mathrm{MA}, \mathrm{P}}\right\rangle$ and $\overline{B_{\mathrm{MA}, \mathrm{P}}}$. However, to evaluate Equations 16a-c, 20a-c, 22, and 25, pathway-specific dose 
correlations noted above were applied (as applicable) to $\mathrm{E} B_{\mathrm{MA}}$ and $B_{\mathrm{MA}}$ to regenerate the pathway-specific variates involved in these equations.

All calculations were performed on a PowerMac G4 computer using the programs Mathematica ${ }^{\circledR} 4.0$ (Wolfram, 1999) and RiskQ 4.0 (Bogen, 2000). Documentation of these calculations appears in Appendices 3.A through 3.I, in which calculations and related comments are organized by topic. Appendices 3.A (Concentration), 3.B (Intakes), and 3.C (Fraction of Lifetime at One Local Residence) all document the derivation or rederivation of exposure-related input variates explained in Daniels et al. (2000), which were used to calculate TCE exposures as explained above (Section 3.2). Appendices 3.D (Effective Genotoxic Dose) and 3.E (Effective Cytotoxic Dose) document the calculation of corresponding biologically effective (TCE or TCA) doses. Note that calculations pertaining to the definition or characterization of variates $V_{\mathrm{W}}, V_{\mathrm{Vmax}}, V_{\mathrm{fm}, \mathrm{ing}}, V_{\mathrm{fd}},\left(f_{\mathrm{deq}} / V_{t, \mathrm{P}}\right)$ and $V_{\mathrm{e}}$ all appear in Appendix 3.E. Appendix 3.F (Effective Dose Correlations) documents calculations made to estimate rank correlations among MA- and pathwayspecific normalized biologically effective doses. Appendix 3.G (Potency) documents all calculations made pertaining to modeled dose-response under both mechanisms of carcinogenic action considered $\left(\mathrm{MA}_{\mathrm{G}}\right.$ and $\mathrm{MA}_{\mathrm{C}}$ ). Appendix 3.H (TCE Risk) documents all calculations made pertaining to corresponding predicted risk. Note that calculations pertaining to the definition of variates $U_{\mathrm{chron}}, U_{\mathrm{tdyn}}$ and $V_{\mathrm{tdyn}}$ appear in Appendix 3.H. Finally, Appendix 3.I (Functions Used) briefly describes all Mathematica ${ }^{\circledR}$ and RiskQ functions used to carry out calculations documented in Appendices 3.A-3.H. More detailed explanation of Mathematica ${ }^{\circledR}$, RiskQ, and JUV analysis is beyond the scope of this report, and is provided in references cited.

All constants and variates defined in this report that were used as input to estimate risk, as described above in Sections $2.1-2.6$, are summarized in the following table (Table 2). 
Table 2. Constants and variates used as input for unified TCE risk assessment. ${ }^{a}$

\begin{tabular}{|c|c|c|c|c|c|c|c|c|}
\hline \multirow{2}{*}{$\begin{array}{l}\text { Input } \\
\text { type }^{b}\end{array}$} & \multirow[b]{2}{*}{$\mathrm{ID}^{b}$} & \multirow[b]{2}{*}{ Description } & \multirow[b]{2}{*}{ Unit } & \multirow{2}{*}{$\begin{array}{c}1^{\text {st }} \text { used } \\
\text { in or near } \\
\text { Equation(s) } \\
\text { (No.) }\end{array}$} & \multicolumn{3}{|c|}{$\begin{array}{l}\text { Distribution type, } \\
\text { parameter value(s) }\end{array}$} & \multirow[b]{2}{*}{ Reference $(\mathrm{s})^{d}$} \\
\hline & & & & & Dist & $\mu$ & $\sigma$ & \\
\hline$K$ & fTCA & Fraction of total TCE intake metabolized to TCA & unitless & 10 & & 0.33 & & Allen \& Fisher (1993) \\
\hline$K$ & MW & TCA to TCE molecular-weight ratio & unitless & 10 & & 1.228 & & Bogen \& Gold (1997) \\
\hline$P$ & $Y_{0}$ & Background relative TBARS level in mouse liver & unitless & 17,18 & & 40 & & Larson \& Bull (1992) \\
\hline$P$ & $Y_{\text {sig }}$ & Significantly elevated relative TBARS level & unitless & 18 & & 49.4 & & Larson \& Bull $(1992)^{*}$ \\
\hline$P$ & $\hat{\mu}, s_{\hat{\mu}}$ & $\begin{array}{l}\text { Estimated location parameter of lognormal } \\
\text { cytotoxicity dose-response model, and its SD }\end{array}$ & unitless & 18 & & 3.05 & 0.0920 & $\begin{array}{l}\text { Calculated from data of } \\
\text { Larson \& Bull (1992) }\end{array}$ \\
\hline$P$ & $\hat{\sigma}, s_{\hat{\sigma}}$ & $\begin{array}{l}\text { Estimated shape parameter of lognormal cytotoxicity } \\
\text { dose-response model, and its SD }\end{array}$ & unitless & 18 & & 0.732 & 0.176 & $\begin{array}{l}\text { Calculated from data of } \\
\text { Larson \& Bull (1992) }\end{array}$ \\
\hline$P$ & $\hat{a}$ & $\begin{array}{l}\text { Estimated log-linear-regression intercept parameter } \\
\text { used to extrapolate cytotoxicity dose-response }\end{array}$ & unitless & 23 & & -7.60 & & Calculated (see text) \\
\hline$P$ & $\hat{b}$ & $\begin{array}{l}\text { Estimated log-linear-regression slope parameter } \\
\text { used to extrapolate cytotoxicity dose-response }\end{array}$ & unitless & 23 & & 3.68 & & Calculated (see text) \\
\hline$u$ & pot & $\begin{array}{l}\text { Carcinogenic potency of TCE assuming a genotoxic } \\
\text { mechanism of action }\end{array}$ & $\mathrm{kg} \mathrm{d} \mathrm{mg}^{-1}$ & 16 & Emp & $3.7 \times 10^{-4}$ & & $\begin{array}{l}\text { Calculated from data on } \\
7 \text { studies (see text) }\end{array}$ \\
\hline$U$ & tdyn & $\begin{array}{l}\text { Uncertainty factor for interspecies extrapolation of } \\
\text { toxicodynamically equivalent effective dose }\end{array}$ & unitless & 16,20 & $\mathrm{LN}$ & 0 & 1.6 & $\begin{array}{l}\text { EPA }(1998)^{*} \\
\text { Slob \& Pieters }(1999)^{*}\end{array}$ \\
\hline$u$ & chron & $\begin{array}{l}\text { Uncertainty factor for extrapolation of acute to } \\
\text { chronic cytotoxic dose }\end{array}$ & unitless & 19 & $\mathrm{LN}$ & $\ln 5$ & $\ln 2.12$ & $\begin{array}{l}\text { NCI }(1976)^{*} \\
\text { Slob \& Pieters }(1999)^{*}\end{array}$ \\
\hline$U$ & $\mathrm{t} 1$ & Normalized estimation error in $\hat{\mu}$ & unitless & 18 & $\mathrm{~T}^{f}$ & 14 & & Assumed \\
\hline$U$ & $\mathrm{t} 2$ & Normalized estimation error in $\hat{\sigma}$ & unitless & 18 & $\mathrm{~T}^{f}$ & 14 & & Assumed \\
\hline$V$ & W & U.S. adult male and female body weight & $\mathrm{kg}$ & 2 & $\mathrm{LN}^{e}$ & 4.24 & 0.221 & $\begin{array}{l}\text { Finley }(1994) \\
\text { CalEPA }(1996, \text { p. 10-7) }\end{array}$ \\
\hline
\end{tabular}


Table 2. Constants and variates used as input for unified TCE risk assessment (continued). ${ }^{\mathrm{a}}$

\begin{tabular}{|c|c|c|c|c|c|c|c|c|}
\hline \multirow{2}{*}{$\begin{array}{l}\text { Input } \\
\text { type }^{b}\end{array}$} & \multirow[b]{2}{*}{$\mathrm{ID}^{b}$} & \multirow[b]{2}{*}{ Description } & \multirow[b]{2}{*}{ Unit } & \multirow{2}{*}{$\begin{array}{c}1^{\text {st }} \text { used } \\
\text { in or near } \\
\text { Equation(s) } \\
\text { (No.) }\end{array}$} & \multicolumn{3}{|c|}{$\begin{array}{l}\text { Distribution type, } \\
\text { parameter value(s) }\end{array}$} & \multirow[b]{2}{*}{ Reference $(\mathrm{s})^{d}$} \\
\hline & & & & & Dist & $\mu$ & $\sigma$ & \\
\hline$V$ & alv & $\begin{array}{l}\text { Normalized variability in alveolar ventilation rate, } \\
\text { independent of variability in } V_{\mathrm{w}}\end{array}$ & unitless & 2,3 & $\mathrm{LN}$ & -0.0409 & 0.286 & CalEPA $(1996$, p. 3-31)* \\
\hline$V$ & $\mathrm{~Pb}$ & Human blood:air partition coefficient for TCE & $\mathrm{L}_{\mathrm{air}} / \mathrm{L}_{\text {blood }}$ & 7 & $\mathrm{~N}$ & 10.2 & 1.6 & Fisher (1998)* \\
\hline$V$ & $\operatorname{liv}$ & Human blood flow to liver $=26 \% \times(15.0 / 12.9) \times V_{\text {alv }}$ & $\mathrm{mL} \mathrm{h}^{-1}$ & 8 & & (see $V_{\text {alv }}$ ) & & Allen \& Fisher (1993) \\
\hline$V$ & $\mathrm{~V}_{\max }$ & $\begin{array}{l}\text { Normalized variability in the maximum rate of TCE } \\
\text { metabolism, independent of variability in } V_{\mathrm{W}}\end{array}$ & unitless & 8 & $\mathrm{LN}$ & -0.152 & 0.551 & Lipscombe et al. (1998)* \\
\hline$V$ & $\mathrm{fd}$ & $\begin{array}{l}\text { Fraction of } V_{\mathrm{W}} \text { corresponding to apparent volume of } \\
\text { distribution for TCA }\end{array}$ & $\mathrm{L} \mathrm{kg}^{-1}$ & 10 & $\mathrm{U}^{e}$ & 0.052 & 0.152 & Allen \& Fisher $(1993)^{*}$ \\
\hline$V$ & e & $\begin{array}{l}\text { Normalized variability in TCA elimination rate } V_{\mathrm{ke}} \\
\text { independent of variability in } V_{\mathrm{w}}\end{array}$ & unitless & 10 & & -0.152 & 0.551 & \\
\hline$V$ & tdyn & $\begin{array}{l}\text { Variability factor modeling intraspecies differences } \\
\text { in sensitivity (i.e., in toxicodynamically equiv. dose) }\end{array}$ & unitless & 16,20 & $\mathrm{LN}$ & 0.700 & 2.33 & $\begin{array}{l}\text { EPA }(1998)^{*} \\
\text { Slob \& Pieters }(1998)^{*}\end{array}$ \\
\hline
\end{tabular}

${ }^{a}$ Constants and variates listed are those defined in this report and used or implied in Equations $1-21$ as inputs to risk estimation. Variates defined by Equations 1-21 are not repeated in this table. All other variates that were used to estimate risk are either defined in Daniels et al. (2000), or are defined in Equations $1-21$ in terms of constants and variates listed in this table.

${ }^{b}$ Input types: $K=$ constant, $P=$ estimated (hence, constant) parameter value, $U=$ uncertain variate, $V=$ heterogeneous variate (i.e., values pertain to different individuals at risk). ID = the subscript that appears in the text on a $K-, U$ - or $V$ type input; $\mathrm{ID}=$ the symbol used in the text to denote a $P$-type input.

'Dist specifies distribution type: $\mathrm{LN}=$ lognormal, $\mathrm{N}=$ standard normal, $\mathrm{T}=$ Student's $\mathrm{T}, \mathrm{U}=\mathrm{uniform}, \mathrm{Emp}=\mathrm{calculated}$ empirical, Blank = not applicable. The values $\{\mu, \sigma\}=\{$ the estimated/assigned value, (if applicable) the SD $\}$ of for $K-$ or $P$ type inputs; otherwise $\{\mu, \sigma\}$ denote (for the specified Dist): $\{\ln G M, \ln G S D\}(L N),\{A M, S D\}(N),\{d f,-\}(T)$, and $\{\min , \max \}(U)$.

${ }^{d}$ An asterisk signifies that the value or approach cited was modified slightly or generalized for use in this report.

ef These variates assumed to have a rank correlation equal to: $-0.50^{e}, 0.294$. 


\section{RESULTS}

Resulting estimates of biologically effective dose TCE contamination at Site LF-13 are presented below in Section 3.1, followed (in Section 3.2) by estimated dose-response relations obtained. Finally, Section 3.3 provides a characterization of corresponding risks and associated JUV estimated using the systematic probabilistic framework adopted for this study, as well as a comparison of these estimates with point-estimates of risk for Site LF-13 obtained using traditional methods.

\subsection{Biologically Effective Dose}

The cdfs obtained to characterize variability in the limiting fraction $V_{\mathrm{fm} \text {,ing }}$ of lowlevel ingested TCE that is metabolized, and in the corresponding limiting fraction $V_{\mathrm{fm}, \text { inh }}$ $\left(=V_{\mathrm{fm}, \mathrm{der}}\right)$ of low-level respired or dermally absorbed TCE that is metabolized, are shown in Figure 3. The variates $\left\{V_{\mathrm{fm}, \mathrm{inh}}, V_{\mathrm{alv}}\right\}$ were found to have an approximate rank correlation of $-0.75(\mathrm{CVM}=0.33 \%)$. Although not used in calculations performed in this study, the rank correlation between variates $\left\{V_{\text {fm,ing, }}, V_{\text {fm,inh }}\right\}$ was found to be $\sim 0.83$ $(\mathrm{CVM}=0.30 \%)$.

The JUV-expectation of genotoxic effective dose, $\left\langle\overline{D_{\mathrm{G}}}\right\rangle$, was found to be $5.93 \times 10^{-5} \mathrm{mg} \mathrm{kg}^{-1} \mathrm{~d}^{-1}(\mathrm{CVM}<1 \%)$, with corresponding pathway-specific dose fractions: $f_{\mathrm{G}, \text { ing }}=0.843, f_{\mathrm{G}, \text { inh }}=0.039$, and $f_{\mathrm{G} \text {,der }}=0.118$. The JUV-expectation of cytotoxic effective dose, $\left\langle\overline{D_{\mathrm{C}}}\right\rangle$, was found to be $0.0269 \mathrm{mg} \mathrm{L}^{-1}(\mathrm{CVM}<1 \%)$, with corresponding pathwayspecific dose fractions: $f_{\mathrm{C}, \text { ing }}=0.604, f_{\mathrm{C} \text {,inh }}=0.312$, and $f_{\mathrm{C}, \text { der }}=0.084$.

The cdfs obtained for the three pathway-specific expectations with respect to uncertainty in normalized effective genotoxic dose $\left(\left\langle B_{G, P}\right\rangle\right.$ for $\mathrm{P}=\{$ ing, inh, der $\}$, shown as three bold curves), and for the corresponding three pathway-specific expectations with respect to variability in normalized effective genotoxic dose $\left(\overline{B_{\mathrm{G}, \mathrm{P}}}\right.$, three light curves), are plotted in Figure 4a. The figure shows that the three pathway-specific curves that comprise each set of (bold or light) curves are virtually indistinguishable. The cdfs obtained for the three pathway-specific expectations with respect to uncertainty in normalized effective cytotoxic dose ( $\left\langle B_{\mathrm{C}, \mathrm{P}}\right\rangle$, three bold curves), and for the corresponding three pathway-specific expectations with respect to variability in 


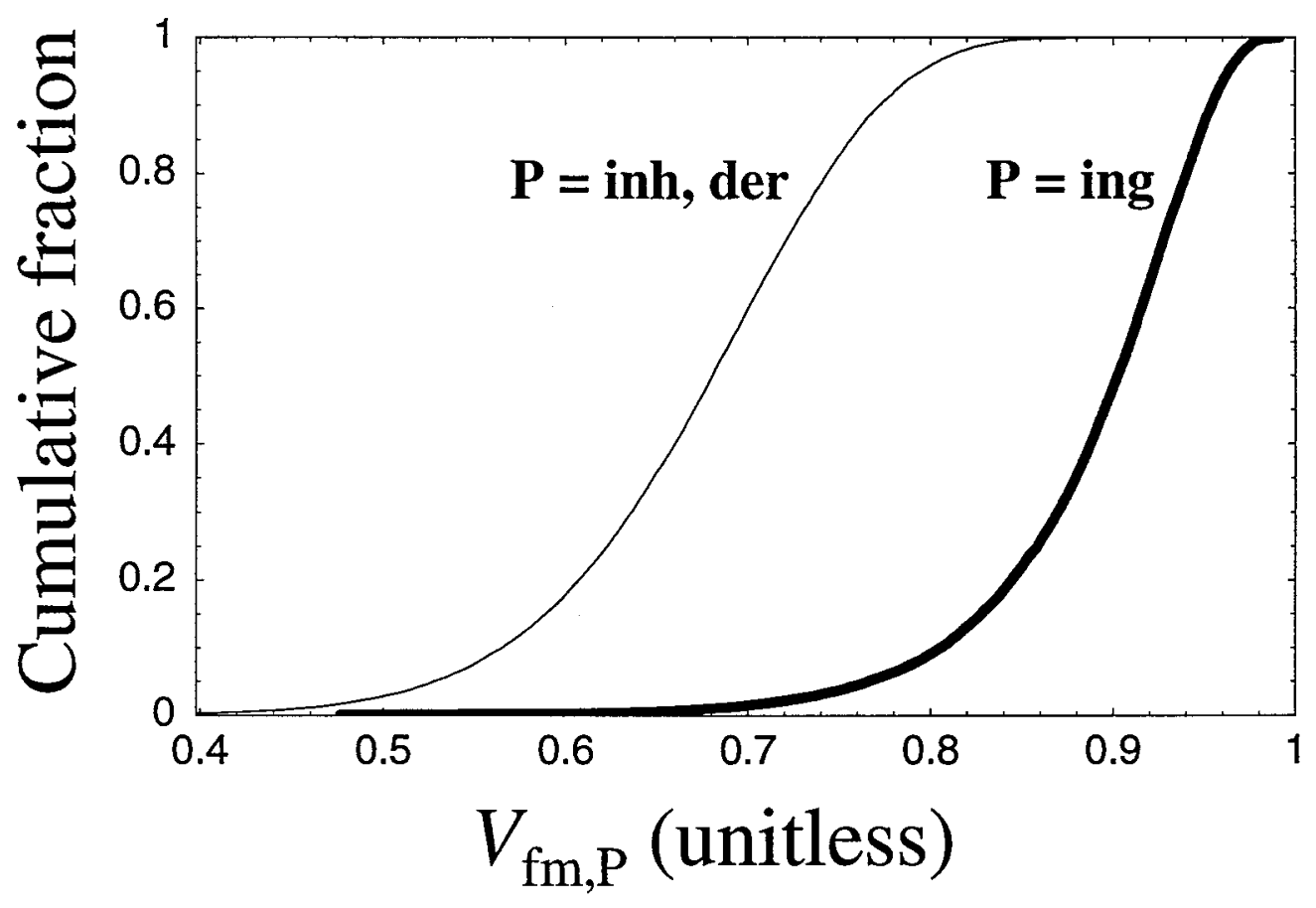

Figure 3. Cumulative distribution functions characterizing interindividual variability in limiting metabolized fractions $V_{\mathrm{fm}, \mathrm{P}}$ of low-level TCE absorbed via different exposure pathways $\mathrm{P}$, where $\mathrm{P}=\{$ ing, inh, and der $\}$ for \{ingestion, ihalation, and dermal\} pathways, respectively. 

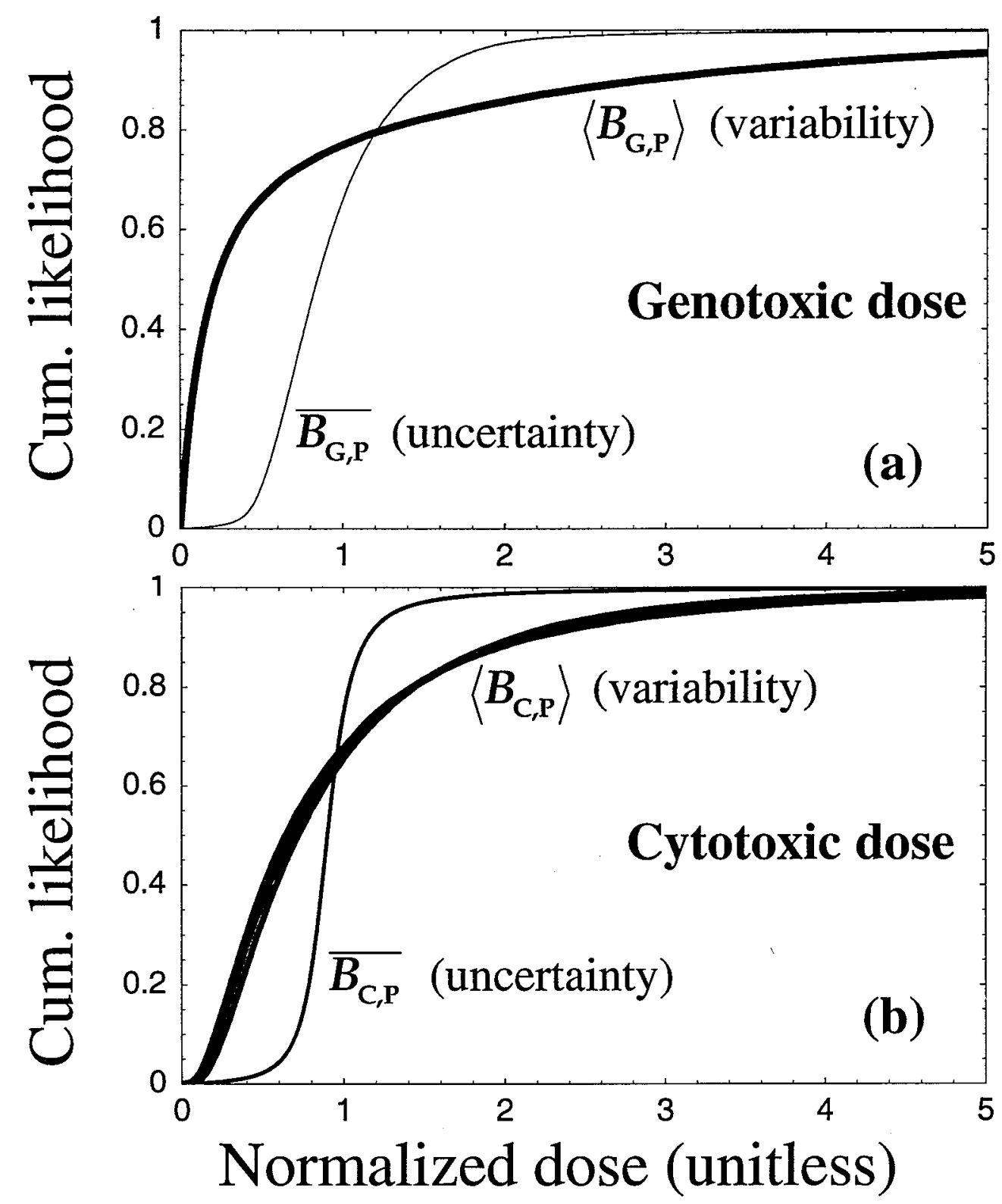

Figure 4. Expectations with respect to uncertainty vs. variability in normalized effective (a) genotoxic dose $\left(B_{\mathrm{G}, \mathrm{P}}\right)$, and (b) cytotoxic dose $\left(B_{\mathrm{C}, \mathrm{P}}\right)$, shown for each $(\mathrm{P}=$ ingestion, inhalation, and dermal) exposure pathway considered. Each corresponding cumulative distribution function ( $\mathrm{cdf}$ ) shown characterizes normalized interindividual variability in values of expected risk (bold curves), or normalized uncertainty in the population-average value of risk (light curves) predicted for hypothetical residents exposed to TCE from ground water at Site LF-13. Three bold and three light exposure-pathway-specific curves appear in each plot, but in each set these curves very nearly coincide (except for slight divergence among bold curves in plot $b$ ). All the cdfs were normalized to have an arithmetic mean value of one. 
normalized effective cytotoxic dose ( $\overline{B_{\mathrm{C}, \mathrm{P}}}$, three light curves), are plotted in Figure $4 \mathrm{~b}$. The figure shows that the three pathway-specific curves that comprise $\overline{B_{\mathrm{C}, \mathrm{P}}}$ (light curves) are virtually indistinguishable, while those comprising $\left\langle B_{\mathrm{C}, \mathrm{P}}\right\rangle$ (bold curves) are nearly so. Thus $\left\langle B_{\mathrm{MA}}\right\rangle \approx\left\langle B_{\mathrm{MA}, \mathrm{P}}\right\rangle$ and $\overline{B_{\mathrm{MA}}} \approx \overline{B_{\mathrm{MA}, \mathrm{P}}}$ for all pathways $\mathrm{P}$, which justifies the exclusive reliance on $\left\langle B_{\mathrm{MA}}\right\rangle$ and $\overline{B_{\mathrm{MA}}}$ for calculations described in Methods. The variates $\left\{\overline{B_{\mathrm{G}}}, \overline{B_{\mathrm{C}}}\right\}$ were found to have an approximate rank correlation of 0.49 $(\mathrm{CVM}=0.67 \%)$, and rank-correlation and corresponding CVM matrices obtained for the $\operatorname{six}\left\langle B_{\mathrm{MA}, \mathrm{P}}\right\rangle$ variates are listed below in Table 3.

\subsection{Dose-Response}

The cdfs characterizing estimation error (uncertainty) in cancer potency $U_{\text {pot }}$ estimated for each of seven animal-bioassay data sets considered are shown in Figure 5a; the corresponding weighted-average cdf based on weights indicated in Table 1 is shown in Figure $5 b$.

The fit of the lognormal model specified by Equation 17 to mouse TBARS-vs. $-A_{\mathrm{C}}$ data is shown in Figure 6. The model fit the experimental data reasonably well $\left(X^{2}=13.6, d f=14, p=0.48\right)$. The two corresponding parameter estimates $( \pm 1 \mathrm{SD})$ $\{\hat{\mu}=3.05 \pm 0.0920, \hat{\sigma}=0.732 \pm 0.176\}$ and their estimated correlation coefficient $(r=0.294)$ were obtained from this fit. The corresponding risk function $F_{\mathrm{C}}\left(D_{\mathrm{Ca}}\right)$ calculated using Equations $24 \mathrm{a}-\mathrm{b}$ is shown in Figure $7 \mathrm{a}$. Figure $7 \mathrm{~b}$ shows the risk function $F_{\mathrm{C}}\left(D_{\mathrm{Ca}}\right)$ replotted on a log-log scale, together with an approximating linearquadratic equation fit to $F_{\mathrm{C}}\left(D_{\mathrm{Ca}}\right)$ as described in Section 2.6: $\log _{10}$ (risk) $=a+b x+c x^{2}$, where $x=\log _{10}\left(D_{\mathrm{Ca}}\right), a=-7.238, b=2.466 x$, and $c=0.4699 x^{2}$. The comparison shown in Figure $7 \mathrm{~b}$ demonstrates that the linear-quadratic equation provides an excellent approximation to $F_{\mathrm{C}}\left(D_{\mathrm{Ca}}\right)$ over a substantial risk range $\left(\sim 10^{-10}\right.$ to $\left.\sim 10^{-2}\right)$ relevant to the present analysis. Figure $7 \mathrm{~b}$ also shows how the slope of the $F_{\mathrm{C}}\left(D_{\mathrm{Ca}}\right)$ function plotted on a log-log scale changes abruptly at a risk level of $\sim 10^{-6}$, implying that a Monte Carlo approach would not be practical as an alternative to using Equations $24 \mathrm{a}-\mathrm{b}$ in order to evaluate levels of cytotoxic risk $F_{\mathrm{C}}\left(D_{\mathrm{Ca}}\right) \leq \sim 10^{-6}$, based on Equation 18 . 
Table 3. Rank correlations among uncertainty-expectations of normalized biologically effective doses. ${ }^{a}$

\begin{tabular}{|c|c|c|c|c|c|c|c|}
\hline \multirow{2}{*}{\multicolumn{2}{|c|}{$\left\langle B_{\mathrm{MA}, \mathrm{P}}\right\rangle$}} & \multicolumn{6}{|c|}{$\left\langle B_{\mathrm{MA}, \mathrm{P}}\right\rangle$ variate } \\
\hline & & MA $\quad \mathbf{P}$ & MA $\quad \mathbf{P}$ & MA $\quad \mathbf{P}$ & MA $\quad \mathbf{P}$ & MA $\quad \mathbf{P}$ & MA $\quad \mathbf{P}$ \\
\hline \multicolumn{2}{|c|}{ variate } & $\mathrm{G} \quad$ ing & $\mathrm{C}$ ing & G inh & C inh & G der & C der \\
\hline $\begin{array}{l}\text { MA } \\
\mathbf{P}\end{array}$ & $\begin{array}{c}\text { G } \\
\text { ing }\end{array}$ & 1 & 0.23 & 0.88 & 0 & 0.89 & 0 \\
\hline $\begin{array}{l}\text { MA } \\
\mathbf{P}\end{array}$ & $\begin{array}{c}\mathrm{C} \\
\text { ing }\end{array}$ & 0.23 & 1 & 0 & 0.42 & 0 & 0.51 \\
\hline $\begin{array}{l}\text { MA } \\
\mathbf{P}\end{array}$ & $\begin{array}{c}\text { G } \\
\text { inh }\end{array}$ & 0.88 & 0 & 1 & 0.19 & 0.92 & 0.035 \\
\hline $\begin{array}{l}\text { MA } \\
\mathbf{P}\end{array}$ & $\begin{array}{l}\text { C } \\
\text { inh }\end{array}$ & 0 & 0.42 & 0.19 & 1 & 0.077 & 0.65 \\
\hline $\begin{array}{l}\text { MA } \\
\mathbf{P}\end{array}$ & $\begin{array}{l}\text { G } \\
\text { der }\end{array}$ & 0.89 & 0 & 0.92 & 0.077 & 1 & 0.18 \\
\hline $\begin{array}{l}\text { MA } \\
\mathbf{P}\end{array}$ & $\begin{array}{l}\text { C } \\
\text { der }\end{array}$ & 0 & 0.51 & 0.035 & 0.65 & 0.18 & 1 \\
\hline
\end{tabular}

${ }^{a}$ Estimated values of the Spearman rank correlation coefficient ( $r$, shown with two significant digits) based on Monte-Carlo evaluation of the uncertainty-expectation of Equation $5 \mathrm{c}$ based on Equations 6 and $15 \mathrm{a}-\mathrm{b}$, where $n_{\text {sam }}=500$ and $n_{\text {sim }}=50$. For all $r$-values listed, $\operatorname{SDM}<0.0025$ where $\operatorname{SDM}=\left(n_{\text {sim }}\right)^{-1 / 2} \operatorname{SD}(r)$ and $\mathrm{SD}(r)$ denotes the $\mathrm{SD}$ of the $n_{\text {sim }}$ estimates of $r$ obtained.

\subsection{Predicted Risk}

The individual risks predicted in this study correspond to the assumption that the assumed TCE concentration in ground water beneath Site LF-13 ( 22 ppb, as of 1997) remains unchanged (see Daniels et al., 2000). The cdfs obtained that characterize uncertainty in the predicted population-average value of individual risk, $\bar{R}$, and interindividual variability in expected values (i.e., "best" estimates) of individual risk, $\langle R\rangle$, are shown in Figures $8 \mathrm{a}$ and $8 \mathrm{~b}$, respectively, plotted together with corresponding CVM values. In Figures $9 \mathrm{a}-\mathrm{c}$, the $\mathrm{cdfs} \bar{R}$ and $\langle R\rangle$ are contrasted over different ranges 


\section{Bioassay-specific potency distributions}

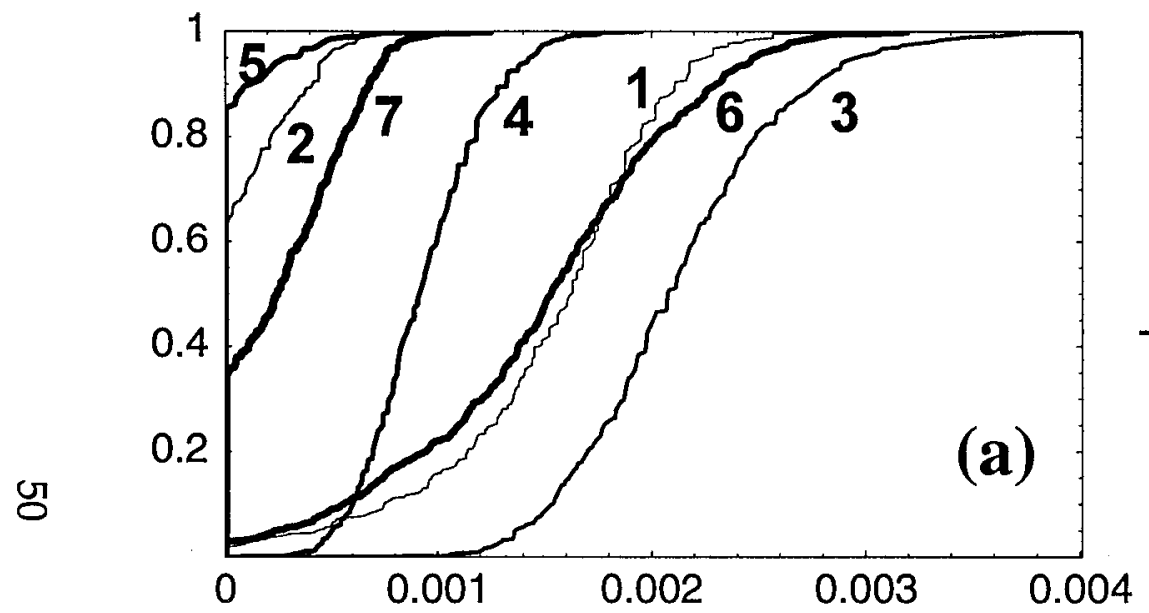

Potency (mg metab. TCE/kg-d)
Weighted average potency distribution

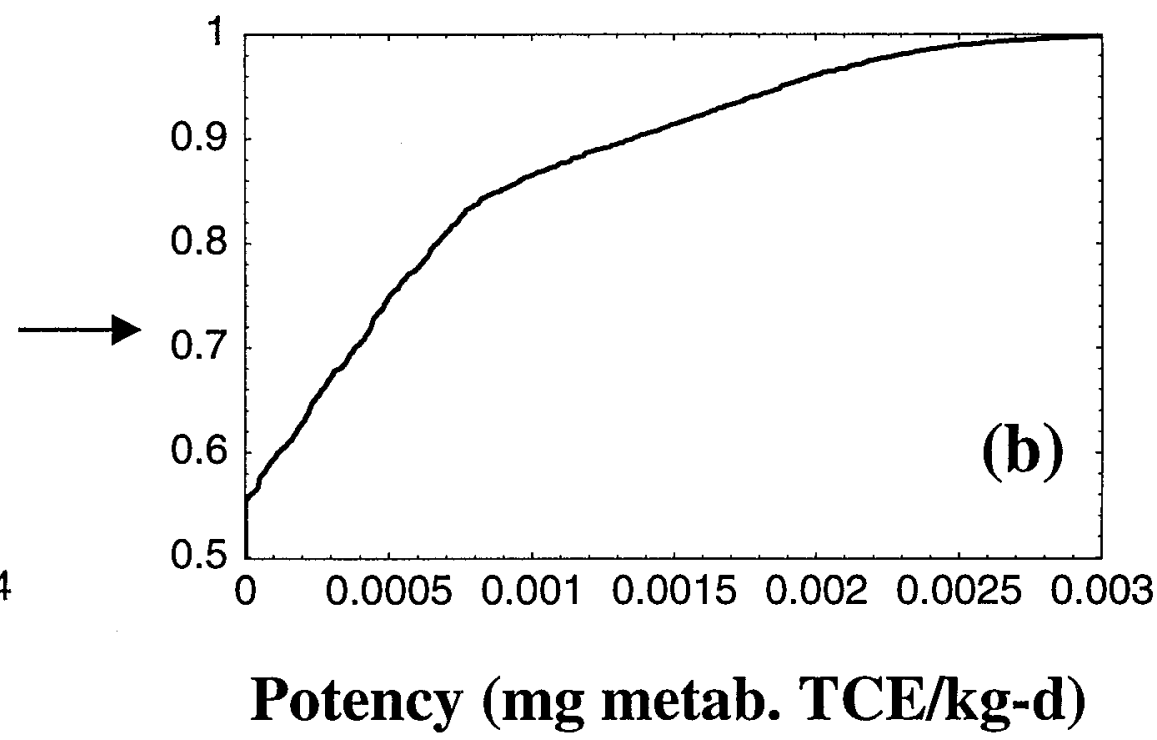

Figure 5. Estimation error (uncertainty) in cancer potency based on rodent-bioassay data. Cumulative distribution functions (cdfs) shown characterize uncertainty in potency estimates based on (a) individual data sets, and (b) a corresponding weighted average of the species/strain/sex-specific cdfs. The numbers labeling individual cdfs in (a) correspond to the study numbers listed in the first column of Table 1. The vertical axis represents cumulative probability. 


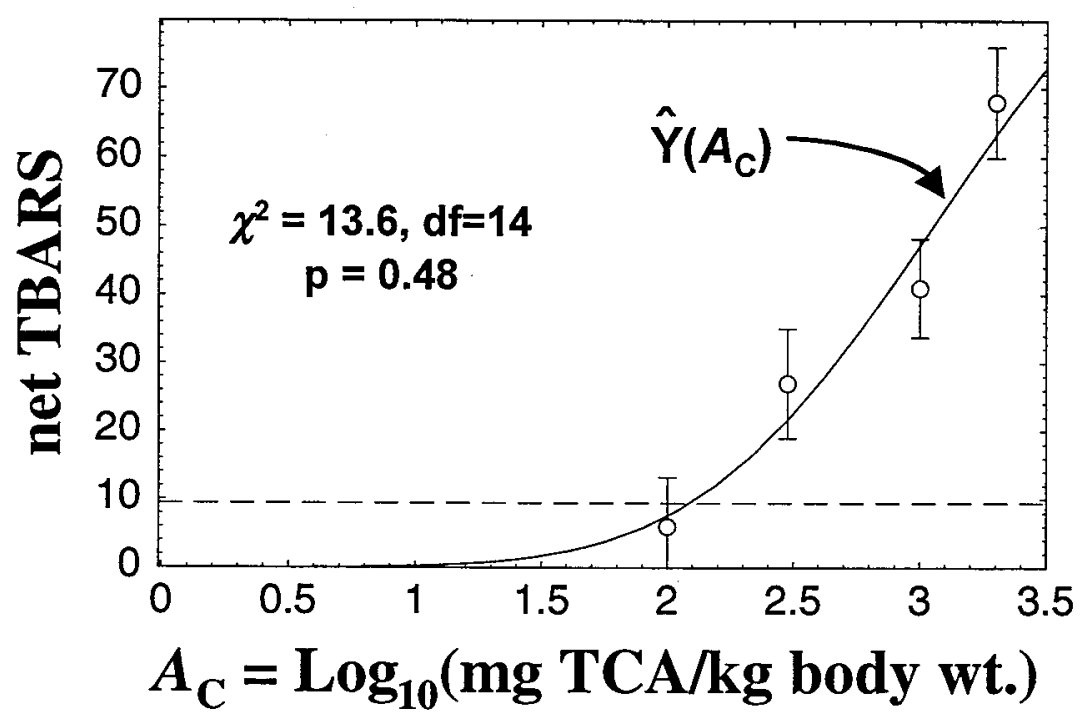

Figure 6. Fit of lognormal model to mous e cytotoxicity data from Larson and Bull (1992a). Experimental mean ( $\pm 1 \mathrm{SD}$ ) data (open points) are shown relating administered TCA dose $\left(A_{C}\right)$ to a hepatocellular lipoperoxidative index (TBARS) associated with liver cytotoxicity, from which the mean $\left(Y_{0}\right)$ of measured control TBARS levels has been subtracted. The dashed line indicates the 2-tailed $95 \%$ upper confidence limit (UCL, $Y_{\mathrm{sig}}$ ) on $Y_{0}$, minus $Y_{0}$. The lognormal model shown (curve; see Equation 17) was fit to the data. 

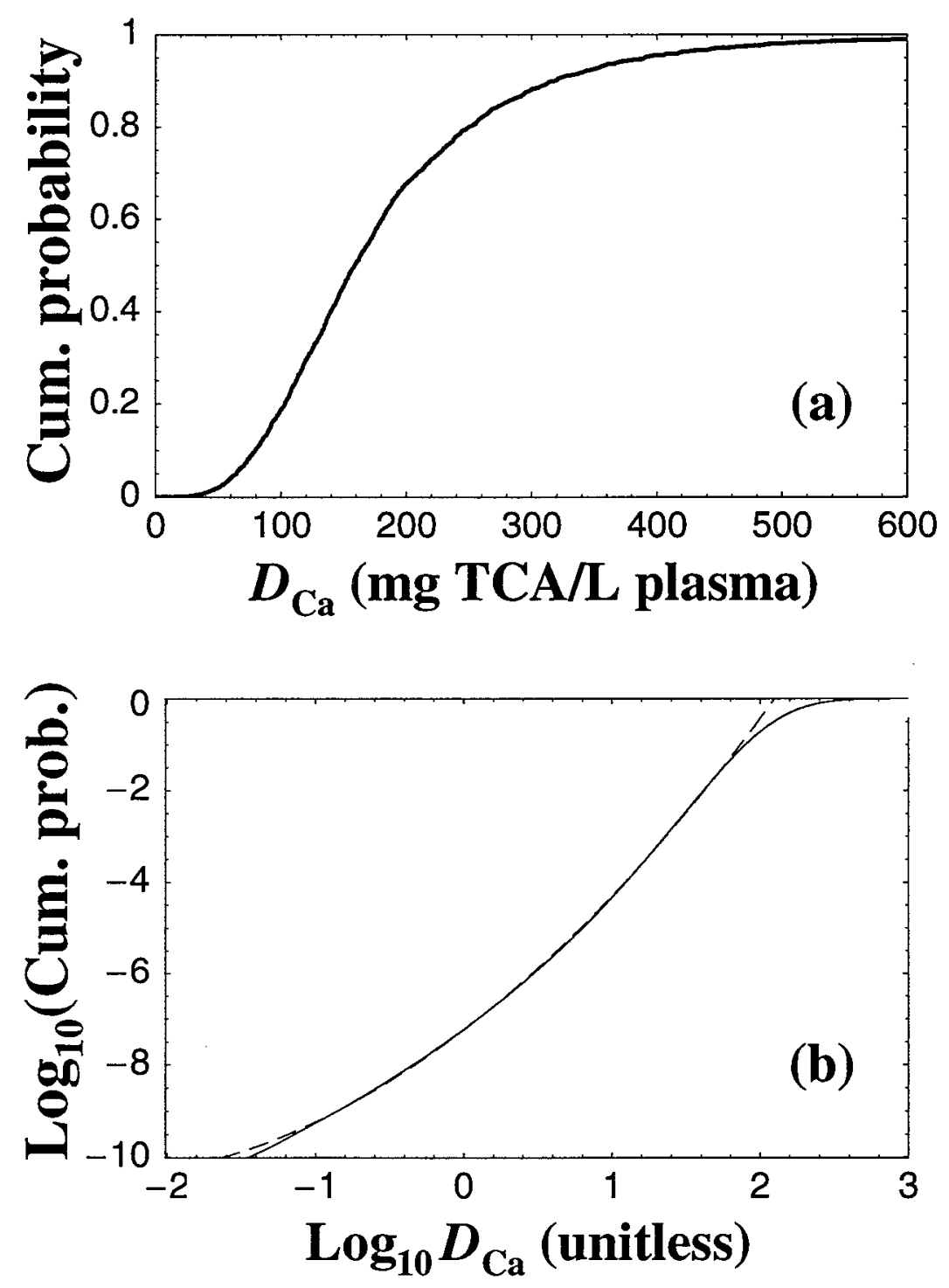

Figure 7. (a) Risk of cytotoxic response, $F_{\mathrm{C}}\left(D_{\mathrm{Ca}}\right)$, as a function of acute effective administered TCA dose $D_{\mathrm{Ca}}$ estimated from mouse cytotoxicity data of Larson and Bull (1992a) on TCA-induced TBARS elevation (see Figure 6). (b) The function $F_{\mathrm{C}}\left(D_{\mathrm{Ca}}\right)$ (solid curve) replotted on a $\log$-log scale, together with the approximating equation, $\log _{10}($ risk $)=-7.238+2.466 x+0.4699 x^{2}$ (dashed curve), where $x=\log _{10}\left(D_{C_{1}}\right)$. 

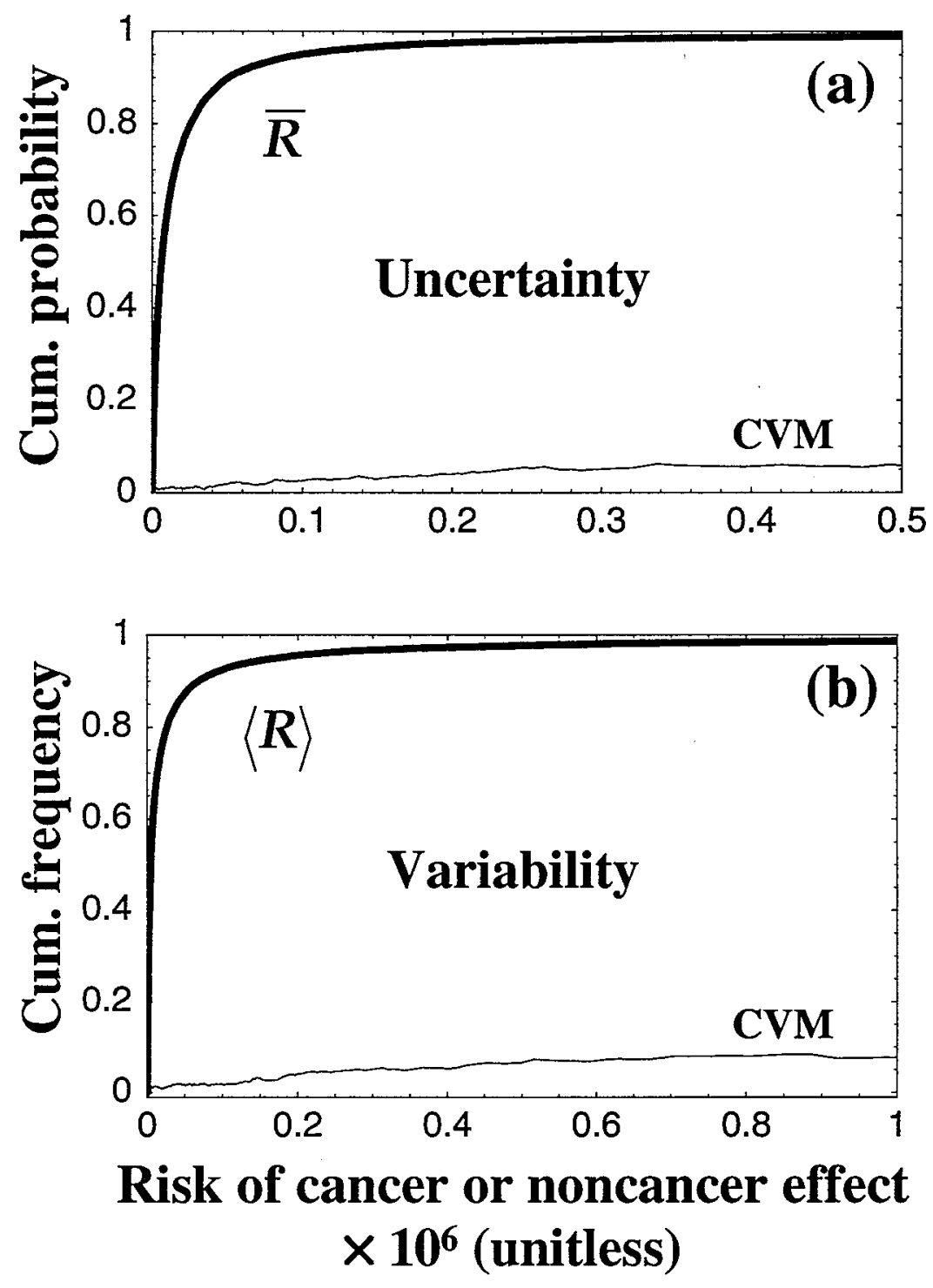

Figure 8. Uncertainty in (a) population-average risk, $\bar{R}$, and (b) interindividual variability in expected risk, $\langle R\rangle$, predicted for hypothetical individuals exposed to TCE in ground water from Site LF-13. In each plot, the Monte-Carlo relativesampling error of the $\mathrm{x}$-axis value of each point on the bold cdf curve is indicated by the corresponding $y$-axis value (labeled CVM) of the light curve shown. For example, from plot (a) the $99^{\text {th }}$ percentile value of $\bar{R}$ is estimated to be $\sim 0.49 \times 10^{-6}$, which estimate has a CVM of $\sim 0.060$, indicating a sampling error of about $\pm 6 \%$. 


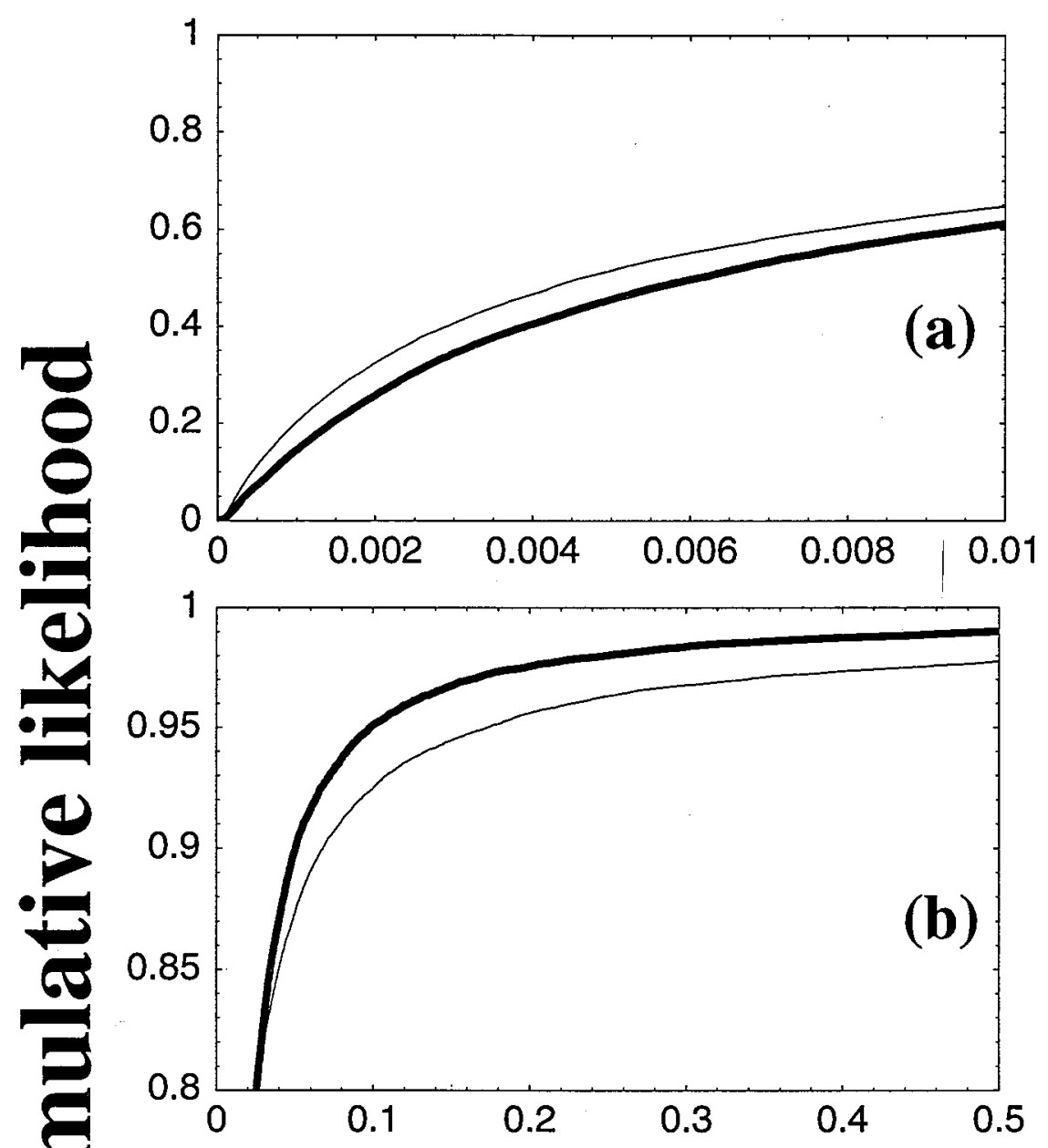

$\Xi$

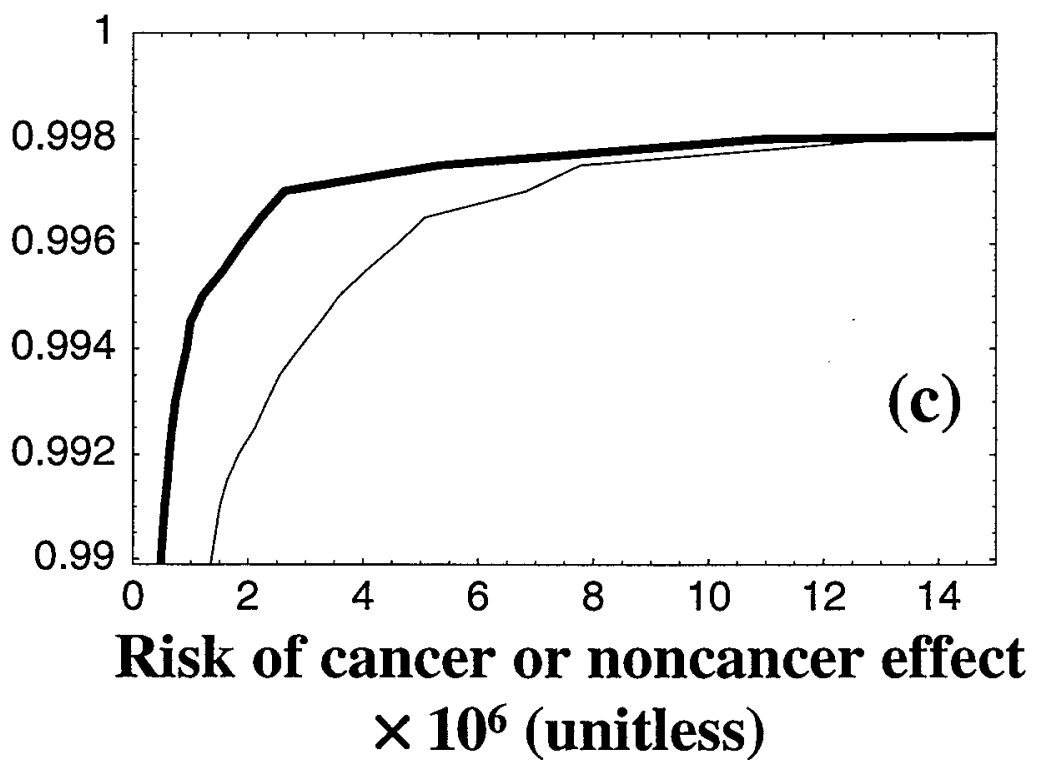

Figure 9. Comparison of $\bar{R}$ (bold curves) vs. $\langle R\rangle$ (light curves) over different ranges of predicted risk: (a) $R \leq 0.01 \times 10^{-6}$, (b) $R \leq 0.5 \times 10^{-6}$, and (c) $R \leq 15 \times 10^{-6}$. The relationship is not consistent over these risk ranges, and both cdfs are highly skewed. 
of risk. Figures 10a-b compare cdfs with respect to variability in JUV estimators $R_{u, v}$ conditional on specified confidence bounds $(u)$ on uncertainty, for $u=0.50,0.95$, and 0.99. The nested Monte-Carlo calculations required to estimate these three $R_{u, v}$ cdfs took a total of $10.6 \mathrm{~h}$ to perform.

Table 4 provides a comparison of an upper variability bound $(v=0.99)$ on these cdfs to mean and upper-bound values of $\bar{R}$ and $\langle R\rangle$, as well as to traditional pointestimates of risk ( $\hat{R}_{\mathrm{RME}}$ and $\left.\hat{R}_{\text {High }}\right)$ taken from Daniels et al. (2000). CVM values were all about $10 \%$ or less, except for a CVM value of $23 \%$ for the expected value of $\bar{R}$. These CVM values indicate reliability in the result obtained that the estimated mean and upper-bound values of $\bar{R}$ and $\langle R\rangle$ are all $\leq \sim 10^{-6}$, and that both JUV estimators are $<$ $5 \times 10^{-5}$.

Corresponding estimates of population risk (i.e., the uncertain number $N$ of cases of cancer or TCE-induced toxicity) depend on the assumed size $n$ of the total exposed population (including all immigrants to and emigrants from areas hypothetically served by Site LF-13 water), for an arbitrarily assumed total period equal to one average lifetime (taken to be $70 \mathrm{y}$ ), during which total or partial lifetime exposures would hypothetically occur (see Daniels et al., 2000). Expected population risk $\langle N\rangle$ conditional on various assumptions concerning population size $n$ are listed in Table 5 , together with estimates of the corresponding likelihood $\left(1-P_{0}\right)$ of one or more cases, and the likelihood $\left(P_{0}\right)$ of zero cases, being attributable to TCE in groundwater at Site LF-13 over the 70-y period of consideration. 


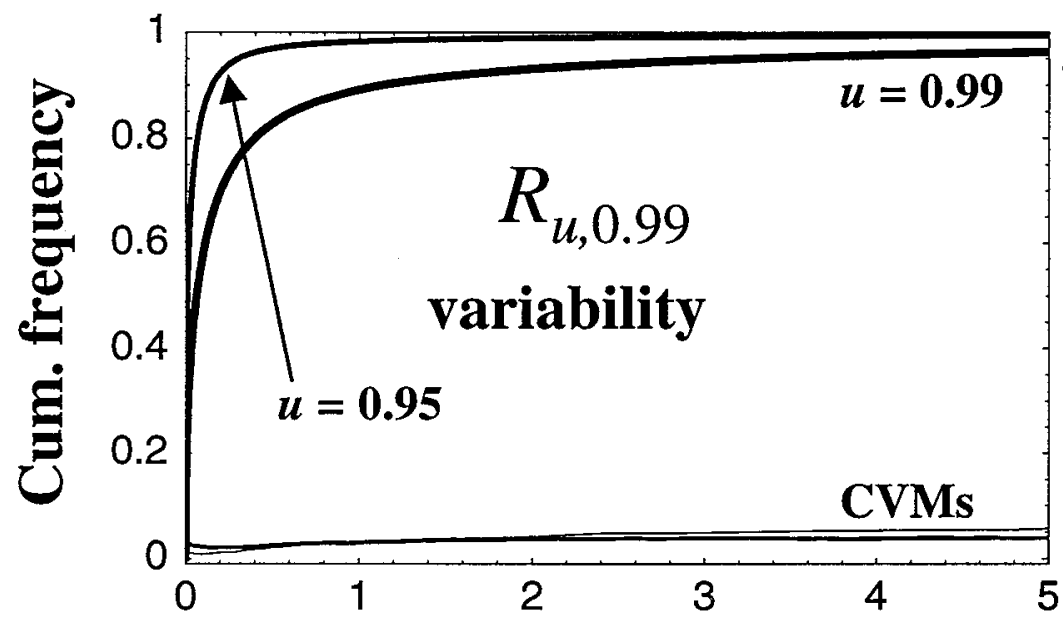

(a)

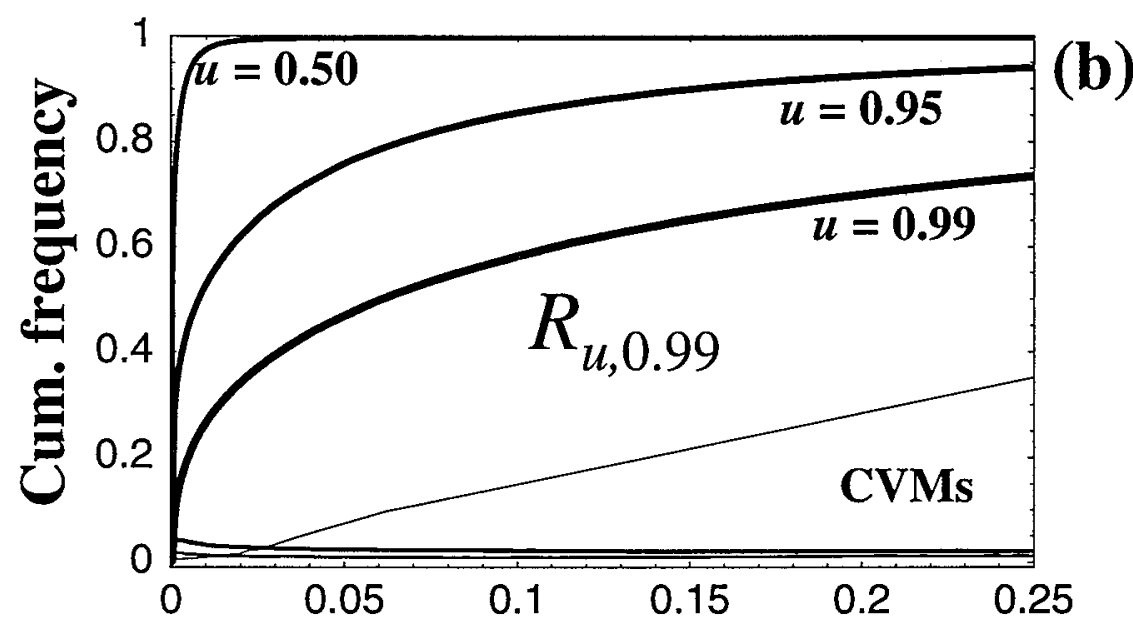

\section{Risk of cancer or noncancer effect $\times 10^{6}$ (unitless)}

Figure 10. Comparison of estimators, $R_{u, v}$ of joint uncertainty and variability in risk, over different ranges fo risk, where $u$ and $v$ refer to fractiles with respect to uncertainty and variability, respectively, and where the upper-bound value $v=0.99$ was used. In (a), $R_{u, 0.99} \leq 5 \times 10^{-6}$, an $d$ in (b) $R_{u, 0.99} \leq 0.25 \times 10^{-6}$. CVM cu rves denote corresponding relative error, as in Figure 8. The CVM that exceeds 0.20 where $R_{u, 0.99}=0.25 \times 10^{-6}$ corresponds to value of $u=0.50$ (median uncertainty), conditional on $w$ hich, for example, $R_{0.5,0.99}=0.017 \times 10^{-6}$. The CVM $>0.20$ therefore pertains to a very unlikely level of risk. 
Table 4. Summary of estimated risk posed by TCE in ground water at Site LF-13.

\begin{tabular}{|c|c|c|c|}
\hline $\begin{array}{l}\text { Method } \\
\quad \text { Type of risk estimator }\end{array}$ & Symbol & $\begin{array}{l}\text { Value } \\
\left(\times 10^{6}\right)\end{array}$ & $\begin{array}{l}\text { Error } \\
(\%)^{*}\end{array}$ \\
\hline \multicolumn{4}{|l|}{ Traditional } \\
\hline Risk to "reasonably maximum exposed" indiv. & $R_{\mathrm{RME}}$ & 61. & NA \\
\hline Upper "conservative" bound (using 95\%UCLs) & $R_{\mathrm{Hi}}$ & 240. & NA \\
\hline \multicolumn{4}{|l|}{ Explicit with respect to: ${ }^{a}$} \\
\hline U in population-average risk $\bar{R}$ & & & \\
\hline Expectation (with respect to $U$ ) & $\langle\bar{R}\rangle$ & 0.92 & 69. \\
\hline Upper $95 \%$ U-bound & $\bar{R}_{0.95}$ & 0.098 & 2.5 \\
\hline Upper $99 \%$ U-bound & $\bar{R}_{0.99}$ & 0.49 & 6.0 \\
\hline$V$ in expected risk $\langle R\rangle$ & & & \\
\hline Population-average (with respect to $\mathrm{V}$ ) & $\overline{\langle R\rangle}$ & 0.23 & 43. \\
\hline Upper $95 \%$ V-bound & $\langle R\rangle_{0.95}$ & 0.17 & 2.8 \\
\hline Upper $99 \%$ V-bound & $\langle R\rangle_{0.99}$ & 1.4 & 8.1 \\
\hline Joint $\mathrm{U}$ and $\mathrm{V}$ in risk: $\quad R$ & & & \\
\hline Upper (95\%-99\%) (U,V)-bound & $R_{0.95,0.99}$ & 2.0 & 4.2 \\
\hline Upper $(99 \%-99 \%)(U, V)-$ bound & $R_{0.99,0.99}$ & 37. & 5.3 \\
\hline
\end{tabular}


Table 5. Population risk associated with multipathway exposures to TCE-contaminated ground water at Beale Air Force Base in California. ${ }^{a}$

\begin{tabular}{|rrccc|}
\hline $\begin{array}{c}\text { Total } \\
\text { exposed } \\
\text { population } \\
\text { over 70 y } \\
n\end{array}$ & $\begin{array}{c}\text { Exposed } \\
\text { population } \\
\text { during 7.6 y } \\
n_{\text {res }}\end{array}$ & $\begin{array}{c}\operatorname{Prob}(N=0)^{b} \\
P_{0}\end{array}$ & $\begin{array}{c}\operatorname{Prob}(N \geq 0)^{b} \\
1-P_{0}\end{array}$ & $\begin{array}{c}\text { Expected value } \\
\text { of } N \\
\langle N\rangle=n \times\langle\bar{R}\rangle\end{array}$ \\
\hline 100 & 11 & 0.999913 & 0.000089 & 0.000092 \\
1,000 & 109 & 0.999423 & 0.00058 & 0.00092 \\
2,000 & 217 & 0.999166 & 0.00083 & 0.0018 \\
10,000 & 1,086 & 0.998468 & 0.0015 & 0.0092 \\
30,000 & 3,257 & 0.997568 & 0.0024 & 0.027 \\
100,000 & 10,857 & 0.995219 & 0.0048 & 0.092 \\
$1,000,000$ & 108,571 & 0.974127 & 0.026 & 0.92 \\
$99,796,500$ & $10,835,049$ & 0.500000 & 0.50 & 91. \\
\hline
\end{tabular}

${ }^{a} N=$ population risk, i.e., the predicted number of cases (i.e., individuals with) a cancer or noncancer endpoint due to exposure to TCE from Site LF-13; $n=$ the total number of individuals assumed to incur the population-average risk $\bar{R}$ within a 70-y period of consideration; $n_{\text {res }}=$ mean number of exposed people at any given moment assumed to be served by ground water from Site LF-13, assuming a mean 7.6-y duration of residence (see Daniels et al., 2000).

${ }^{b}$ Probabilities $P_{0}$ are shown rounded to 6 decimal places; complimentary probabilities $\left(1-P_{0}\right)$ are shown rounded to 2 decimal places; and exposed population $n_{\text {res }}$ is shown rounded to the nearest integer; no more than 2 significant digits are implied in estimates listed in columns 2 through 5 . 


\section{DISCUSSION}

A systematic probabilistic framework was used to estimate the aggregate risk of cancer and noncancer endpoints for hypothetical future residents exposed to TCE from ground water at an inactive landfill site at Beale U.S. Air Force Base. The framework used here differs from previous approaches (Baird et al., 1996; Butterworth and Bogdanffy, 1999; Carlson-Lynch et al., 1999; Gaylor et al., 1999; Lewis, 1993; Slob and Pieters, 1998) in that it is the first to provide an integrated, consistent treatment of: (i) cancer as well as noncancer endpoints, (ii) two disparate yet plausible mechanisms of carcinogenic action in the case TCE (genotoxic vs. cytotoxic), (iii) pharmacokinetic considerations, and (iv) quantitative analysis of JUV in model inputs and corresponding characterized risk. The framework incorporates some of the probabilistic methods suggested previously, but modifies others in important and/or fundamental ways. In particular, the human-variability factor $V_{\text {tdyn }}$ (that has an AM of 1) and the acute-tochronic uncertainty factor $\left(1+U_{\text {chron }}\right)$ (that by definition is $>1$ ) used here differs fundamentally from analogous factors recommended by Slob and Pieters (1998), in order for these factors to fit logically within a systematic probabilistic framework that addresses points (i)-(iv) above. The proposed framework is also the first ever to address both issues (i) and (ii) for the purpose of quantitative risk assessment, independent of issues (iii) and (iv). Specifically, the systematic probabilistic framework used in this case study enabled the application of Equation 22, which in turn represents a fundamentally novel way of addressing multiple toxic endpoints and/or alternative modeling assumptions in quantitative risk assessment.

The application of the proposed systematic probabilistic framework to a case involving TCE also illustrates several methods useful for addressing a number of technical issues necessary to consider in order to perform quantitative risk assessment for combined cancer and noncancer endpoints within a systematic probabilistic framework. For example, the quantitative analysis of interindividual variability in pharmacokinetic relations for TCE done in this case study involved a variety of methods that might also be useful in similar analyses for other compounds exhibiting saturable metabolism. Another example is the application of Equations 24a-b to calculate a dose-vs.-risk-of-adverse-response cdf that reflects uncertainty in the estimated parameters of an assumed dose-response function fit to available data. These 
equations allow an analytic approach to this problem in the case of approximately normally distributed data, in contrast to Monte Carlo methods (e.g., as proposed by Slob and Pieters, 1998) that may not be practical to implement reliably, as shown in this case study (see Section 3.2).

All estimates of individual risk obtained in this study (Table 4) are far less than corresponding upper-bound point estimates of individual risk, $\hat{R}_{\mathrm{RME}}$ and $\hat{R}_{\mathrm{High}}$, that were obtained for comparison by Daniels et al. (2000) using standard, traditional deterministic methods (namely, algebraic substitution of upper-bound and/or default parameter values into equations used to estimate risk). Daniels et al. (2000), who did not consider JUV in pharmacokinetic and dose-response relations pertaining to TCE risk, obtained a similar result. In the present study, the systematic probabilistic framework used to consider JUV in pharmacokinetic and dose-response relations pertaining to TCE had a substantial impact on predicted risk for Site LF-13. This impact can be assessed by comparing the risk summary in Table 4 of the present study with that in Table 3 of Daniels et al. (2000). The upper-bound risk estimators $\bar{R}_{0.95}$ and $\langle R\rangle_{0.95}$ obtained in the present study are about 60- and 80-fold less, respectively, than the value of these estimators obtained by Daniels et al. (2000).

Even the JUV-estimator $R_{0.95,0.95}$ approximated in Daniels et al. (2000) is (slightly) greater than the value (of $\sim 2 \times 10^{-6}$ ) obtained here using a nested Monte-Carlo procedure for the more conservative JUV-estimator $R_{0.95,0.99}$. Only the even more conservative JUV-estimator, $R_{0.99,0.99}$, obtained $\left(37 \times 10^{-6}\right)$ was substantially greater than $10^{-6}$. While the latter value is less than the deterministically (hence, relatively easily) calculated $\hat{R}_{\mathrm{RME}}$ value of $\left(\sim 60 \times 10^{-6}\right)$, the two values are fairly close, indicating that $\hat{R}_{\mathrm{RME}}$ in this case provides a credible estimate of the more precisely defined estimator $R_{0.99,0.99}$ (namely, the $99^{\text {th }}$ percentile on uncertainty in risk to the person who is at the $99^{\text {th }}$ percentile of risk relative to others at risk). Furthermore, $\hat{R}_{\mathrm{RME}}$ is relatively easily calculated, whereas $R_{0.99,0.99}$ required about $10 \mathrm{~h}$ of computation in the present study. It might therefore be preferable to use $\hat{R}_{\mathrm{RME}}$ to optimize risk reduction relative to an upper-bound JUV estimator (such as $R_{0.99,0.99}$ ). 
Upper-bound JUV estimators allow explicit consideration of equity in the distribution of interindividual variability in imposed risk. Point estimates such as $\hat{R}_{\mathrm{RME}}$ cannot do this explicitly, because they cannot generally be interpreted in any precise manner with respect to variability per se or to uncertainty per se. In the present case study, both $\hat{R}_{\mathrm{RME}}$ and $R_{0.99,0.99}$ are $<5 \times 10^{-4}$, which indicates (vaguely via $\hat{R}_{\mathrm{RME}}$, explicitly by $R_{0.99,0.99}$ ) the de minimis nature of predicted upper-bound risks plausibly due to TCE at Site LF-13 hypothetically faced by those who would be among most at risk relative to others exposed to ground water from that site. However, such risk estimates do not necessarily correspond to the magnitude of health consequences predicted to be associated with such exposure. Such effects can only be addressed by quantitatively considering uncertainty in population risk, which in turn can only be accomplished by quantitative JUV analysis that characterizes uncertainty in population-average risk $\bar{R}$ conditional on population size $n$ (e.g., via Equation 22) (Bogen, 1986,1990b; Bogen and Spear, 1987).

Interesting results concerning population risk were obtained in this study via quantitative JUV analysis addressing multiple health endpoints and multiple mechanisms concerning TCE-induced health risk. Earlier results by Daniels et al. (2000) indicated that exposure to TCE from ground water at Site LF-13 would be unlikely to cause a single occurrence of a TCE-related health impact provided that $n<\sim 30,000$. Results from the present, more comprehensive analysis (Table 5) indicate that a single case most likely would not occur even if the number of people served by ground water from Site LF-13 were ten million. That is, the new results indicate that a single case is unlikely to occur under any realistic assumption concerning population size. Moreover, results obtained in the present study indicate that, under the assumptions used, there is

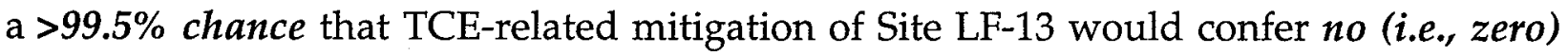
public-health benefit if as many as 10,000 or fewer people were residentially served by the site's TCE-contaminated water (assuming the concentration of TCE never were to increase above levels measured in 1997). Therefore, under this population scenario, any resources directed at mitigating the site are virtually certain to be wasted from a publichealth perspective. Even this hypothetical scenario is conservative, because it is likely that current TCE contamination in ground water at Site LF-13 (due to a finite mass of TCE contamination) could not persist for $70 \mathrm{y}$ if that water were to serve 10,000 
hypothetical residents throughout this period. Dilution of the source mass is expected, and the magnitude of this dilution is expected to be proportional to the water flow rate into and away from the source; indeed, this is the basis for pump-and-treat sitemitigation strategies. Water service to that many people from this single goundwater source - if even possible-would also induce some (perhaps substantial) infiltration of non-contaminated water, again causing dilution of residentially delivered TCE concentrations. 


\section{REFERENCES}

Abbas, R., and J.W. Fisher. 1997. A physiologically based pharmacokinetic model for trichloroethylene and its metabolites, chloral hydrate, trichloroacetate, dichloroacetate, trichloroethanol, and trichloroethanol glucuronide in B6C3F1 mice. Toxicol. Appl. Pharmacol. 147:15-30.

Acharya, S., K. Mehta, S. Rodriguez, J. Pereira, S. Krishnan, and C.V. Rao. 1997. A histopathological study of liver and kidney in male Wistar rats treated with subtoxic doses of t-butyl alcohol and trichloroacetic acid. Exper. Toxicol. Pathol. 49:369-373.

Aitchison, J., and J.A.C. Brown. 1957. The Lognormal Distribution. Cambridge University Press, New York.

Allen, B.C., and J.W. Fisher. 1993. Pharmacokinetic modeling of trichloroethylene and trichloroacetic acid in humans. Risk Anal. 15:71-86.

Ames, B., and L.S. Gold. 1990a. Too many rodent carcinogens: Mitogenesis increases mutagenesis. Science. 249:970-972.

Ames, B.N., and L.S. Gold. 1990b. Chemical carcinogenesis: Too many rodent carcinogens. Proc. Natl. Acad. Sci. 87:7772-7776.

Ames, B.N., L.S. Gold, and W.C. Willett. 1995. The causes and prevention of cancer. Proc. Natl. Acad. Sci. 92:5258-5265.

Ames, B.N., M.K. Shigenaga, and L.S. Gold. 1993. DNA lesions, inducible DNA repair, and cell division: Three key factors in mutagenesis and carcinogenesis. Environ. Health Perspect. 101(suppl. 5):35-44.

Andersen, M.E., H.A. Barton, R. Bull, and I. Schultz. 1998. DCA dosimetry: Interpreting DCA-induced liver cancer dose-response and the potential for DCA to contribute to TCE-induced liver cancer, AL-OE-DR-TR-1998-0009. United States Air Force Armstrong Laboratory, Brooks Air Force Base, TX.

Anderson, E.L., R.E. Albert, R. McGaughy, L. Anderson, S. Bayard, D. Bayliss, C. Chen, M. Chu, H. Gibb, B. Haberman, C. Hiremath, D. Singh, and T. Thorslund. 1983. Quantitative approaches in use to assess cancer risk. Risk Anal. 3:277-295.

Armitage, P., and R. Doll. 1957. A two-stage theory of carcinogenesis in relation to the age distribution of human cancer. Br. J. Cancer. 11:161-169.

Baird, S.J.S., J.T. Cohen, J.D. Graham, A.I. Shlyakter, and J.S. Evans. 1996. Noncancer risk assessment: A probabilistic alternative to current practice. Human Ecol. Risk Assessment. 2:79-102.

Bell, Z.G., K.J. Olsen, and T.J. Benya. 1978. Final Report of Audit Findings of the Manufacturing Chemists Association (MCA): Administered Trichloroethylene (TCE) Chronic Inhalation Study at Industrial Bio-Test Laboratories, Inc., Decator, Illinois. Unpublished study reported in USEPA (1985).

Bogen, K.T. 1986. Uncertainty in Environmental Health Risk Assessment: A Framework for Analysis and an Application to a Chronic Exposure Situation Involving a Chemical Carcinogen. Doctoral dissertation. University of California, Berkeley, School of Public Health. Berkeley, CA. 
Bogen, K.T. 1988. Pharmacokinetics for regulatory risk assessment: The case of trichloroethlyene. Regulatory Toxicol. Pharmacol. 8:447-466.

Bogen, K.T. 1989. Cell proliferation kinetics and multistage cancer risk models. J. National Cancer Inst. 81:267-277.

Bogen, K.T. 1990a. Risk extrapolation for chlorinated methanes as promoters vs initiators of multistage carcinogenesis. Fund. Appl. Toxicol. 15:536-557.

Bogen, K.T. 1990b. Uncertainty in Environmental Health Risk Assessment. Garland Publishing, Inc., New York.

Bogen, K.T. 2000. RiskQ 4.0: An Interactive Approach to Probability, Uncertainty and Statistics for use with Mathematica ${ }^{\circledR}$. UCRL-MA-110232 Rev. 1. Lawrence Livermore National Laboratory, Livermore, CA.

Bogen, K.T. 1994. Cancer potencies of heterocyclic amines found in cooked foods. Fd. Chem. Toxicol. 32:505-515.

Bogen, K.T. 1995. Methods to approximate joint uncertainty and variability in risk. Risk Anal. 15:411-419.

Bogen, K.T., and L.S. Gold. 1997. Trichloroethylene cancer risks: Simplified calculation of PBPK-based MCLs for cytotoxic endpoints. Regulatory Toxicol. Pharmacol. 25:2642.

Bogen, K.T., and L.C. Hall. 1989. Pharmacokinetics for regulatory risk assessment: The case of 1,1,1-trichloroethane (Methyl chloroform). Regulatory Toxicol. Pharmacol. 10:26-50.

Bogen, K.T., L.C. Hall, T.E. McKone, D.W. Layton, and S.E. Patton. 1988. Health Risk Assessment of Trichloroethylene in California Drinking Water. Report prepared for the California Public Health Foundation and California Department of Health Services. UCRL-21007. Lawrence Livermore National Laboratory, Livermore, CA.

Bogen, K.T., and R.C. Spear. 1987. Integrating uncertainty and interindividual variability in environmental risk assessment. Risk Anal. 7:427-436.

Brown, L.P., D.G. Farrar, and C.G. DeRooij. 1990. Health risk assessment of environmental exposure to trichloroethylene. Regulatory Toxicol. Pharmacol. 11:2441.

Bull, R.J., I.M. Sanchez, M.A. Nelson, J.L. Larson, and A.J. Lansing. 1990. Liver tumor induction in $\mathrm{B} 6 \mathrm{C} 3 \mathrm{~F} 1$ mice by dichloroacetate and trichloroacetate. Toxicology. 63:341-359.

Bull, R.J. 2000. Mode of action of liver tumor induction by trichloroethylene and its metabolites, trichloroacetate and dichloroacetate. Environ. Health Perspect. 108(suppl. 2): 241-259.

Butterworth, B.E., and M.S. Bogdanffy. 1999. A comprehensive approach for integration of toxicity and cancer risk assessments. Regulatory Toxicol. Pharmacol. 29:23-36.

California Environmental Protection Agency (CalEPA). 1996. Air Toxics Hot Spots Program Risk Assessment Guidelines Part IV, Technical Support Document: Exposure Assessment and Stochastic Analysis (December 1996). CalEPA Office of Health Hazard Assessment, Berkeley, CA. 
Carlson-Lynch, H., P.S. Price, J.C. Swartout, M.L. Dourson, and R.E. Keenan. 1999. Application of quantitative information on the uncertainty in the RfD of Noncarcinogenic risk assessments. Human Ecol. Risk Assess. 5:527-546.

Chaubey, Y.P., and G.S. Mudholkar. 1982. A new approximation for the distribution of the difference of two t-variables. Commun. Statist.-Theor. Meth. 11:2335-2342.

Cohen, S.M., and L.B. Ellwein. 1990. Cell proliferation in carcinogenesis. Science. 249:1007-1011.

Cohen, S.M., and L.B. Ellwein. 1991. Genetic errors, cell proliferation, and carcinogenesis. Cancer Res. 51:6493-6505.

Daniels, J., K.T. Bogen, and L. Hall. 1999. Procedures for Addressing Uncertainty and Variability in Exposure to Characterize Potential Health Risk From Trichloroethylene Contaminated Groundwater at Beale Air Force Base in California. UCID-CR-135784, Rev. 1. Lawrence Livermore National Laboratory, Livermore, CA.

Daniels, J., K.T. Bogen, and L. Hall. 2000. Analysis of uncertainty and variability in exposure to characterize risk: Case study involving trichloroethylene groundwater contamination at Beale Air Force Base in California. Water, Air, and Soil Pollution 123:273-298.

DeAngelo, A.B., F.B. Daniel; B.M. Most, and G.R. Olson. 1997. Failure of monochloroacetic acid and trichloroacetic acid administered in the drinking water to produce liver cancer in male F344/N rats. J. Toxicol. Environ. Health. 52:425-445.

DeAngelo, A.B., F.B. Daniel, J.A. Stober, and G.R. Olsen. 1991. The carcinogenicity of dichloroacetic acid in the male B6C3F1 mouse. Fund. Appl. Toxicol. 16:337-347.

Dees, C., and C. Travis. 1994. Trichloroacetate stimulation of liver DNA synthesis in male and female mice. Toxicol. Lett. 70:343-355.

Dourson, M.L., S.P. Felter, and D. Robinson. 1996. Evolution of science-based uncertainty factors in noncancer risk assessment. Regulatory Toxicol. Pharmacol. 24:108-120.

Eyre, R.J., D.K. Stevens, J.C. Parker, and R.J. Bull. 1995. Renal activation of trichloroethylene and S-(1,2-dichlorovinyl)-L-cysteine and cell proliferative responses in the kidneys of F344 rats and B6C3F1 mice. J. Toxicol. Environ. Health. 46:465-481.

Fahrig, R., S. Madle, and H. Baumann. 1995. Genetic toxicology of trichloroethylene. Mutat. Res. 340:1-36.

Finley, B., D. Proctor, P. Scott, N. Harrington, D. Paustenback, and P. Prince. 1994. Recommended distributions for exposure factors frequently used in health risk assessment. Risk Anal. 14:533-553.

Fisher, J.W., and B.C. Allen. 1993. Evaluating the risk of liver cancer in humans exposed to trichloroethylene using physiological models. Risk Anal. 15:87-95.

Fisher, J.W., M.L. Gargas, B.C. Allen, and M.E. Andersen. 1991. Physiologically based pharmacokinetic modeling with trichloroethylene and its metabolite, trichloroacetic acid, in the rat and the mouse. Toxicol. Appl. Pharmacol. 109:183-195. 
Fisher, J.W., D. Mahle, and R. Abbas. 1998. A human physiologically based pharmacokinetic model for trichloroethylene and its metabolites, trichloroacetic acid and free trichloroethanol. Toxicol. Appl. Pharmacol. 152:339-359.

Gaylor, D.W., R.L. Kodell, J.J. Chen, and D. Krewski. 1999. A unified approach to risk assessment for cancer and noncancer endpoints based on benchmark doses. Regulatory Toxicol. Pharmacol. 29:151-157.

Ghosh, B.K. 1975. On the distribution of the difference of two $t$-variables. J. Am. Statist. Assoc. 70:463-467.

Harrington-Brock, K., C.L. Doerr, and M.M. Moore. 1998. Mutagenicity of three disinfection by-products: Di- and trichloroacetic acid and chloral hydrate in L5178Y/TK +/- (-)3.7.2C mouse lymphoma cells. Mutat. Res. 413:265-276.

Herren-Freund, S.L., M.A. Pereira, M.D. Khoury, and G. Olson. 1987. The carcinogenicity of trichloroethylene and its metabolites, trichloroacetic and dichloroacetic acid, in mouse liver. Toxicol. Appl. Pharmacol. 90:183-189.

Iman, R.L., and W.J. Conover. 1982. A distribution-free approach to inducing rank correlation among input variates. Commun. Statist. (Ser. B) Simulat. Computat. 11:311-334.

Jennrich, R.I. 1970. An asymptotic $\chi^{2}$ test for the equality of two correlation matrices. J. Am. Statist. Assoc. 65:904-912.

Larson, J.L., and R.J. Bull. 1992a. Metabolism and lipoperoxidative activity of trichloroacetate and dichloroacetate in rats and mice. Toxicol. Appl. Pharmacol. 115:268-277.

Larson, J.L., and R.J. Bull. 1992b. Species differences in the metabolism of trichloroethylene to the carcinogenic metabolites trichloroacetate and dichloroacetate. Toxicol. Appl. Pharmacol. 115:278-285.

Lash, L.H., J.W. Fisher, J.C. Lipscomb, and J.C. Parker. 2000a. Metabolism of trichloroethylene. Environ. Health Perspect. 108(suppl. 2):177-200.

Lash, L.H., J.C. Parker, and C.S. Scott. 2000b. Modes of action of trichloroethylene for kidney tumorigenesis. Environ. Health Perspect. 108(suppl. 2):225-240.

Lewis, S.C. 1993. Reducing uncertainty with adjustment factors: Improvements in quantitative noncancer risk assessment. Fund. Appl. Toxicol. 20:2-4.

Lipscomb, J.C., J.W. Fisher, P.D. Confer, and J.Z. Byczkowski. 1998. In vitro to in vivo extrapolation for trichloroethylene metabolism in humans. Toxicol. Appl. Pharmacol. 152:376-387.

Maltoni, C., G. Lefemine, and G. Cotti. 1986. Archives of Research on Industrial Carcinogenesis. Vol. V. Experimental Research of Trichloroethylene Carcinogenesis. Princeton University Press, Princeton, NJ.

Merdink, J.L., A. Gonzalez-Leon, R.J. Bull, and I.R. Schultz. 1998. The extent of dichloroacetate formation from trichloroethylene, chloral hydrate, trichloroacetate, and trichloroethanol in B6C3F1 mice. Toxicol. Sci. 45:33-41.

Moolgavkar, S.H. 1983. Model for human carcinogenesis: Action of environmental agents. Environ. Health Perspect. 50:285-291. 
Moolgavkar, S.H., A. Dewanji, and D.J. Venzon. 1988. A stochastic two-stage model for cancer risk assessment: The hazard function and the probability of tumor. Risk Anal. 8:383-392.

Moolgavkar, S.H., and A.G. Knudson. 1981. Mutation and cancer: A model for human carcinogenesis. J. Natl. Cancer Inst. 66:1037-1052.

Moore, M.M., and K. Harrington-Brock. 2000. Mutagenicity of trichloroethylene and its metabolites: implications for the risk assessment of trichloroethylene. Environ. Health Perspect. 108(suppl. 2):215-223.

National Cancer Institute (NCI). 1976. Carcinogenesis Bioassay of Trichloroethylene. NCI-CG-TR-2, DHEW Publ. No. (NIH) 76-802. U.S. Government Printing Office, Washington, DC.

National Research Council (NRC). 1994. Science and Judgment in Risk Assessment. NRC Committee on Risk Assessment of Hazardous Air Pollutants, National Academy Press, Washington, DC.

National Toxicology Program (NTP). 1988. Toxicology and Carcinogenesis Studies of Trichloroethylene (CAS No. 79-01-6) in Four Strains of Rats (ACI, August, Marshall, Osborne-Mendel) (Gavage Studies). NIH Pub No. 88-2529, NTP Tech. Rep. Ser. No. 273. National Institutes of Health, NTP, Research Triangle Park, NC.

National Toxicology Program (NTP). 1990. Carcinogenesis Studies of Trichloroethylene (Without Epichlorohydrin) (CAS No. 79-01-6) in F344/N Rates and B3C3F1 Mice (Gavage Studies). NIH Pub No. 90-1799. National Institutes of Health, NTP, Research Triangle Park, NC.

Ni, Y.C., T.Y. Wong, R.V. Llyoyd, T.M. Heinze, S. Shelton, D. Caciano, F.F. Kadlubar, and P.P. Fu. 1996. Mouse liver microsomal metabolism of chloral hydrate, trichloroacetic acid, and trichloroethanol leading to induction of lipid peroxidation via a free radical mechanism. Drug Metab. Disposition 24:81-90.

Ojo, M.O. 1988. Approximation to the distribution of the difference of two weighted and unweighted t-variables. J. Statist. Res. (Bangladesh) 22:37-42.

Patil., V.H. 1965. Approximation to the Behrens-Fisher distributions. Biometrika 52:267271.

Pereira, M.A. 1996. Carcinogenic activity of dichloroacetic acid and trichloroacetic acid in the liver of female B6C3F1 mice. Fund. Appl. Toxicol. 31:192-199.

Pereira, M.A., and J.B. Phelps. 1996. Promotion by dichloroacetic acid and trichloroacetic acid of $N$-methyl- $N$-nitrosourea-initiated cancer in the liver of female B6C3F1 mice. Cancer Lett. 102:133-141.

Purrier. W. 1997. Personal communication regarding detailed data for TCE groundwater concentration at Site LF-13 of Beale Air Force Base, California. Law Engineering and Environmental Services, Inc., Sacramento, CA (December 1997).

Press, W.H., S.A. Teukolsky, W.T. Vetterling, and B.P. Flannery. 1992. Numerical Recipes in FORTRAN - The Art of Scientific Computing. Cambridge University Press, New York, NY, pp. 650-700.

Renwick, A.G. 1993. Data derived safety factors for the evaluation of food additives and environmental contaminants. Food Add. Contam. 10:275-305. 
Rubin, H. 1960. On the distribution of the weighted difference of two independent Student variables. J. Roy. Statist. Soc., Ser. B 22:188-194.

Singh, K.P. 1990. Computing probabilities for the difference of two t-variables. In: Page, C., and R. LePage, eds. Computing Science and Statistics, Statistics of Many Parameters: Curves, Images, Spatial Models. Proceedings of the 22nd Symposium on the Interface, East Lansing, MI, May 16-19, 1990. Springer-Verlag, New York, NY, pp. 519-523.

Slob, W., and M.N. Pieters. 1998. A probabilistic approach for deriving acceptable human intake limits and human health risks from toxicological studies: General framework. Risk Anal. 18:787-798.

Stenner, R.D., J.L. Merdink, J.W. Fisher, and R.J. Bull. 1998. Physiologically-based pharmacokinetic model for trichloroethylene considering enterohepatic recirculation of major metabolites. Risk Anal. 18:261-269.

Templin, M.V., J.C. Parker, and R.J. Bull. 1993. Relative formation of dichloroacetate and trichloroacetate from trichloroethylene in male B6C3F1 mice. Toxicol. Appl. Pharmacol. 123:1-8.

U.S. Environmental Protection Agency (USEPA). 1985. Health Assessment Document for Trichloroethylene. EPA/600/8-82/006F. USEPA Office of Research and Development, Office of Health and Environmental Assessment, Environmental Criteria and Assessment Office, Research Triangle Park, NC.

U.S. Environmental Protection Agency (USEPA). 1987a. Technical Analysis of New Methods and Data Regarding Dichloromethane Hazard Assessments. EPA/600/887/029A (June 1987). USEPA Office of Health and Environmental Assessment, Washington, DC.

U.S. Environmental Protection Agency (USEPA). 1987b. Addendum to the Health Assessment Document for Trichloroethylene: Updated Carcinogenicity Assessment for Trichloroethylene. EPA/600/8-82/006FA. USEPA Office of Research and Development, Office of Health and Environmental Assessment, Environmental Criteria and Assessment Office, Research Triangle Park, NC.

U.S. Environmental Protection Agency (USEPA). 1992. Draft report: A cross-species scaling factor for carcinogen risk. assessment based on equivalence of $\mathrm{mg} / \mathrm{kg}^{3 / 4} /$ day. Fed. Register. 57(No. 109, June 5):24152-24172.

U.S. Environmental Protection Agency (USEPA). 1996. Proposed Guidelines for Carcinogen Risk Assessment. EPA/600/P-92/003C. USEPA Office of Research and Development, Washington, DC.

U.S. Environmental Protection Agency (USEPA). 1998. Draft Water Quality Criteria Methodology: Human Health. Federal Register Notice. [Fed. Regist. 63(No. 157, Aug. 14):43755-43828] EPA/822-Z-98-001. USEPA Office of Water, Washington, DC.

URS Greiner Woodward Clyde (URSGWC). 1998. Management Action Plan, Beale Air Force Base, California. December 1998. Prepared for Headquarters Air Combat Command (ACC), Langley Air Force Base, VA, under contract to US Air Force Center for Environmental Excellence (AFCEE), Brooks Air Force Base, TX, Project No. ACCH19987544 [obtain from Chief, Environmental Restoration (M.E. O'Brien), Beale Air Force Base, California]. URSGWC, Omaha, NE. 
Walker, G.A., and J.G. Saw. 1978. The distribution of linear combinations of $t$-variables. J. Am. Statist. Assoc. 73:876-878.

Weil, C.S. 1972. Statistics vs. safety factors and scientific judgment in the evaluation of safety for man. Toxicol. Appl. Pharmacol. 21:454-463.

Wolfram, S. 1999. The Mathematica Book. Cambridge University Press, Cambridge, UK.

Wright, S. 1992. Adjusted p-values for simultaneous inference. Biometrics. 48:1005-1013. 


\section{Appendix 1}

\section{Method of Moments for Lognormal Variates}

Given a normally distributed variate $Y$ with arithmetic mean (AM) $\mu_{Y}$, standard deviation (SD) $\sigma_{Y}$, and corresponding coefficient of variation ( $\left.\mathrm{CV}=\mathrm{SD} / \mathrm{AM}\right)$ $\gamma_{Y}=\left(\sigma_{Y} / \mu_{Y}\right)$, the variate $X=e^{Y}$ has a lognormal (LN) distribution with geometric mean $(\mathrm{GM}) e^{\mu_{Y}}$ and geometric standard deviation (GSD) $e^{\sigma_{Y}}$, where $e=\ln ^{-1}(1)$ and $\ln$ denotes natural logarithm. These assumptions are efficiently denoted $Y \sim \mathrm{N}\left(\mu_{Y}, \sigma_{Y}\right)$ and $X \sim \mathrm{LN}\left(\mu_{Y}, \sigma_{Y}\right)$. The method of moments may be used to relate given AM, SD, and CV values of $X\left(\mu_{X}, \sigma_{X}\right.$, and $\gamma_{X}$, respectively) to those of $Y$; in particular, the AM/GM ratio for $X, \rho=\left(\mu_{X} / e^{\mu_{Y}}\right)$, is equal to $e^{\sigma_{Y}^{2} / 2}$ where $\sigma_{Y}^{2}=\ln \rho^{2}=\ln \left(1+\gamma_{X}^{2}\right)$ (Aitchison and Brown, 1957).

LN moment relations conveniently imply that the ratio of any given percentile of $X$ relative to its GM or AM corresponds to a unique set of LN parameters. Let $X_{p}=e^{\mu_{Y}+\sigma_{Y} z_{p}}$ denote the 100pth percentile of $X$, where $0 \leq p \leq 1, z_{p}=\Phi^{-1}(p)$, and $\Phi$ is the cumulative normal probability distribution function. Now let $q_{p}=X_{p} / e^{\mu_{Y}}$ and $r_{p}=X_{p} / \mu_{X}$ denote the ratios of $X_{p}$ to the GM and AM of $X$, respectively. Conditional on $e^{\mu_{Y}}$ and the ratio $q_{p}$, it follows immediately (by solving for $\mu_{Y}$ and $\sigma_{Y}$ ) that $X \sim \operatorname{LN}\left(\mu_{Y}, \ln q_{p}^{1 / z_{p}}\right)$. Conditional on $\mu_{X}$ and the ratio $r_{p}$, it follows that $X \sim \mathrm{LN}\left[\ln \left(\mu_{X}\right)-\left(\sigma_{Y}^{2} / 2\right), \sigma_{Y}\right]$, where $\sigma_{Y}$ is the positive $\sigma_{Y}$-root of $\sigma_{Y}^{2}-2 z_{p} \sigma_{Y}+2 \ln r_{p}=0$; i.e., $\sigma_{Y}=z_{p}+\sqrt{z_{p}^{2}-\ln r_{p}^{2}}$ for all $r_{p} \leq e^{z_{p}^{2}} / 2$ (larger values of $r_{p}$ are not possible conditional on $z_{p}$ ).

LN moment relations also imply that for any independent $L N$ variates $X_{i}=e^{Y_{i}}$, $i=1, \ldots, n$, with corresponding CVs $\gamma_{i}$, the CV $\gamma_{Z}$ of the product $Z=\prod_{i=1}^{n} X_{i}$ is conveniently ralated as follows to the CVs $\gamma_{i}$ of $X_{i}$ :

$$
\gamma_{Z}=\sqrt{e^{\sigma_{\ln Z}^{2}}-1}=\sqrt{-1+\exp \left(\sigma_{\sum_{i=1}^{n} Y_{i}}^{2}\right)}
$$




$$
\begin{aligned}
& =\sqrt{-1+\exp \left(\sum_{i=1}^{n} \sigma_{Y_{i}}^{2}\right)}=\sqrt{-1+\exp \left(\sum_{i=1}^{n} \ln \left(1+\gamma_{i}^{2}\right)\right)} \\
& =\sqrt{-1+\prod_{i=1}^{n}\left(1+\gamma_{i}^{2}\right)} .
\end{aligned}
$$

Conditional on known $\gamma_{Z}$ and $\gamma_{i}$ for $i \neq j$ and $1 \leq j \leq n$, inverting the latter equation readily yields the unknown $C V$ of $X_{j}$ as

$$
\gamma_{j}=\sqrt{\frac{1+\gamma_{z}^{2}}{\prod_{i \neq j}^{n}\left(1+\gamma_{i}^{2}\right)}-1}
$$

If $\gamma_{j}$ is obtained in this way and a single additional $X_{j}$-parameter among the set $A \mathrm{AM}$, $\mathrm{SD}, \mathrm{GM}, \mathrm{GSD}$ \} is known, all three remaining $X_{j}$-parameters are easily obtained via the moment relations described above. For example, if the AM of $X_{j}$ equals 1 , it follows that $X_{j} \sim \mathrm{LN}\left(-\sigma^{2} / 2, \sigma\right)$ where $\sigma^{2}=\ln \left(1+\gamma_{j}^{2}\right)$. 


\section{Appendix 2 \\ The Exact Distribution of a Linear Function of Correlated $t$-Distributed Variates}

Exact and corresponding approximate expressions are available for the distribution of linear functions of independent $t$-distributed variates (Ruben, 1960; Patil, 1965; Ghosh, 1975; Walker and Saw, 1978; Chaubey and Mudholkar, 1982; Ojo, 1988; Singh, 1990), but not for correlated $t$-distributed variates. The exact distribution of a particular weighted difference (or sum) of two independent (uncorrelated) $t$-distributed variates, known as the Behrens-Fisher distribution, was derived by Ruben (1960) in integral form. A linear combination of $t$-distributed variates is referred to as a generalized Behrens-Fisher distribution (Patil, 1965). The result obtained by Ruben (1960) is thus one type of generalized Behrens-Fisher distribution. However, his approach can be generalized as follows to obtain the exact distribution of any linear combination of $t$-variates with specified correlations.

Let $S=\sum_{i=1}^{n} w_{i} T_{f_{i}}$ be a general linear function consisting of a weighted sum of correlated, $t$-distributed variates $T_{f_{i}}$, each with corresponding weights $w_{i}$ and degrees of freedom $f_{i}$ for $i=1, \ldots, n$. Now introduce two corresponding sets of auxiliary variates $Z_{i}$ and $U_{i}(i=1, \ldots, n)$, where $Z_{i}$ are correlated $N(0,1)$ (standard normal) variates all with zero mean, unit variance, and an ( $n \times n$ symmetric) correlation matrix $\mathbf{R}=\left(\rho_{i, j}\right)(\{i, j\}=$ $1, \ldots, n)$, and where $U_{i}$ have independent chi-square distributions with $f_{i}$ corresponding degrees of freedom. Below, $g_{X}(x)$ and $G_{X}(x)$ denote the density function and cumulative distribution function (cdf), respectively, of the specified variate $(X)$. It follows that

$$
S \quad=\sum_{i=1}^{n} \frac{w_{i} Z_{i}}{\sqrt{U_{i} / f_{i}}}
$$

so that, conditional on variates $\mathbf{U}=\left(U_{1}, \ldots, U_{n}\right), S$ is distributed as $N\left(0, \sigma_{s}^{2}\right)$, with zero mean, with variance

$$
\sigma_{s}^{2}=\sum_{i=1}^{n} \frac{w_{i}^{2} f_{i}}{U_{i}}+2 \sum_{i<j} \rho_{i, j} w_{i} w_{j} \sqrt{\frac{f_{i} f_{j}}{U_{i} U_{j}}},
$$


and with the corresponding density function,

$$
g_{s}(s \mid \mathbf{U})=\frac{1}{\sqrt{2 \pi} \sigma_{s}} \exp \left(\frac{-s^{2}}{2 \sigma_{s}^{2}}\right)
$$

The unconditional density function of $S$ is then given by

$$
g_{s}(s)=\int_{0}^{\infty} \ldots \int_{0}^{\infty} g_{s}(s \mid \mathbf{u}) \prod_{i=1}^{n} \frac{\mathrm{e}^{\frac{-1}{2} u_{i}} u_{i}^{\left(\frac{f_{i}}{2}-1\right)}}{2^{\frac{1}{2} f_{i}} \Gamma\left(\frac{1}{2} f_{i}\right)} \mathrm{d} u_{i}
$$

Now transform $u_{i}$ in terms of new variates $y=\sum_{i=1}^{n} u_{i}$ and $x_{i}=u_{i} / y$ for $i=1, \ldots, n-1$, and also define $x_{n}=1-\sum_{i=1}^{n-1} x_{i}$ and $\phi^{2}(\mathbf{x})=f /\left(y \sigma_{s}^{2}\right)$, where $\mathbf{x}=\left(x_{1}, \ldots, x_{n-1}\right)$ and $f=\sum_{i=1}^{n} f_{i}$. This transformation implies that

$$
\phi^{2}(\mathbf{x})=\frac{f}{y \sigma_{s}^{2}}=f\left(\sum_{i=1}^{n} \frac{w_{i}^{2} f_{i}}{x_{i}}+2 \sum_{i<j} \rho_{i, j} w_{i} w_{j} \sqrt{\frac{f_{i} f_{j}}{x_{i} x_{j}}}\right)^{-1},
$$

and it has the Jacobian matrix: $\mathbf{J}=\left(y \mathbf{I}_{n}+\mathbf{M}\right)$, where $\mathbf{I}_{n}$ is an $n \times n$ identity matrix and the elements $m_{i, j}$ of the $n \times n$ matrix $\mathbf{M}$ are all zero, except $m_{i, n}=-y$ for $i=1, \ldots, n-1$ and $m_{n, j}=x_{j}$ for $j=1, \ldots, n$. The corresponding Jacobian, $|\mathrm{J}|=\operatorname{Det}(\mathrm{J})=y^{n-1}$, and Equation 2.5, reduce Equation 2.4 to

$$
\begin{aligned}
g_{s}(s) & =\int_{0}^{1} \ldots \int_{0}^{1}\left[\int_{0}^{\infty} g_{s}(s \mid \mathbf{u}) y^{n-1} \mathrm{e}^{\left(\frac{-y}{2}\right)} y^{\left(\frac{f}{2}-n\right)} \mathrm{d} y\right] 2^{\left(\frac{f}{2}\right)} \prod_{i=1}^{n} \frac{x_{i}^{\left(\frac{f}{2}-1\right)}}{\Gamma\left(\frac{1}{2} f_{i}\right)} \mathrm{d} x_{1} \ldots \mathrm{d} x_{n-1}, \\
& =\int_{0}^{1} \ldots \int_{0}^{1}\left[\int_{0}^{\infty} \mathrm{e}^{\frac{-y}{2}\left(1+\frac{s^{2} \phi^{2}(x)}{f}\right)} y^{\left(\frac{f}{2}-1\right)} \mathrm{d} y\right] \frac{\phi(\mathbf{x})}{\sqrt{f}} 2^{\left(\frac{f}{2}\right)} \prod_{i=1}^{n} \frac{x_{i}^{\left(\frac{f}{2}-1\right)}}{\Gamma\left(\frac{1}{2} f_{i}\right)} \mathrm{d} x_{1} \ldots \mathrm{d} x_{n-1} \\
& =\int_{0}^{1} \ldots \int_{0}^{1}\left\{\frac{\left[1+\left(s^{2} \phi^{2}(\mathbf{x}) / f\right)\right]^{\frac{1}{2}(f-1)}}{\sqrt{f} \mathrm{~B}\left(\frac{f}{2}, \frac{1}{2}\right)} \phi(\mathbf{x})\right\} \Gamma\left(\frac{f}{2}\right) \prod_{i=1}^{n} \frac{x_{i}^{\left(\frac{f}{2}-1\right)}}{\Gamma\left(\frac{1}{2} f_{i}\right)} \mathrm{d} x_{1} \ldots \mathrm{d} x_{n-1},
\end{aligned}
$$

in which $\phi(x)$ denotes the positive square root of $\phi^{2}(x)$ defined by Equation 2.5. A new transformation, $t^{\prime}=s \phi(\mathbf{x})$ with Jacobian $J=1 / \phi(\mathbf{x})$, shows the braced expression in 
Equation $2.6 \mathrm{c}$ to be $J$ times the density $g_{T_{f}}\left(t^{\prime}\right)$ of a $t$-variate with $f$ degrees of freedom (i.e., $T_{j}$ ) conditional on $\mathbf{x}$. Integrating Equation 2.6c with respect to $t^{\prime}$ over the range $[-\infty, t]$ shows the cdf of $S$ to involve a multiple integral with respect to $x$ over the cdf for $T_{j}$ evaluated at $\mathbf{x}$-dependent arguments defined using Equation 2.5:

$$
G_{S}(t)=\int_{0}^{1} \ldots \int_{0}^{1} G_{T_{f}}(t \phi(\mathbf{x}) \mid \mathbf{x}) \Gamma\left(\frac{f}{2}\right) \prod_{i=1}^{n} \frac{x_{i}^{\left(\frac{f}{2}-1\right)}}{\Gamma\left(\frac{1}{2} f_{i}\right)} \mathrm{d} x_{1} \ldots \mathrm{d} x_{n-1} .
$$

In the case that $n=2$, using $x$ to denote $x_{1}$, Equation 2.7 reduces to

$$
\begin{aligned}
G_{S}(t) & =\int_{0}^{1} G_{T_{f}}(t \phi(x) \mid x) \frac{x^{\frac{f_{2}}{2}-1}(1-x)^{\frac{f_{2}}{2}-1}}{\mathrm{~B}\left(\frac{f_{1}}{2}, \frac{f_{2}}{2}\right)} \mathrm{d} x \\
& =\int_{0}^{1} G_{T_{f}}(t \phi(x) \mid x)\left(\mathrm{dB}\left(\frac{f_{1}}{2}, \frac{f_{2}}{2}, x\right) / \mathrm{d} x\right) \mathrm{d} x,
\end{aligned}
$$

where $\mathrm{B}(a, b)$ and $\mathrm{B}(a, b, x)$ denote the beta function, and the corresponding incomplete beta-function ratio with respect to $x$, respectively, of the specified arguments (see Abbreviations and Notation). Thus, in this case, $G_{S}(t)$ is for given $t$ the mean value of the conditional Student- $t \mathrm{cdf}, G_{T_{j}}(t \varphi(x) \mid x)$, where $x$ is Beta-distributed with parameters $f_{1} / 2$ and $f_{2} / 2$. The latter fact was noted by Ruben (1960), who obtained $G_{S}(t)$ specifically for the case in which $n=2, w_{1}=\sin \theta, w_{2}=\cos \theta$, and $\rho_{1,2}=0$. 


\section{Appendix 3}

\section{Documentation of Mathematica $4.0^{\circledR}$ and Risk $Q$ Calculations}

All calculations were performed on a 400-MHz PowerMac G4 using the programs Mathematica ${ }^{\circledast} 4.0$ (Wolfram, 1999) and RiskQ 4.0 (Bogen, 2000). Documentation of these calculations appears in Appendices 3.A through 3.I which follow, in which calculations and related comments are organized by topic. Appendices 3.A (Concentration), 3.B (Intakes), and 3.C (Fraction of Lifetime at One Local Residence) all document the derivation or re-derivation of exposure-related input variates explained in Daniels et al. (2000), which were used to calculate TCE exposures as explained above (Section 2.2). Appendices 3.D (Effective Genotoxic Dose) and 3.E (Effective Cytotoxic Dose) document the calculation of corresponding biologically effective (TCE or TCA) doses. Note that calculations pertaining to the definition or characterization of variates $V_{\mathrm{W}}$, $V_{\text {Vmax }}, V_{\text {fm,ing }} V_{\text {fd }},\left(f_{\text {deq }} / V_{t, \mathrm{p}}\right)$ and $V_{\mathrm{e}}$ all appear in Appendix 3.E. Appendix 3.F (Effective Dose Correlations) documents calculations made to estimate rank correlations among MA- and pathway-specific normalized biologically effective doses. Appendix 3.G (Potency) documents all calculations made pertaining to modeled dose-response under both mechanisms of carcinogenic action considered $\left(\mathrm{MA}_{\mathrm{G}}\right.$ and $\left.M \mathrm{~A}_{\mathrm{C}}\right)$. Appendix 3.H (TCE Risk) documents all calculations made pertaining to corresponding predicted risk. Note that calculations pertaining to the definition of variates $U_{\text {chron }}, U_{\text {tdyn }}$ and $V_{\text {tdyn }}$ appear in Appendix 3.H. Finally, Appendix 3.I (Functions Used) briefly describes all Mathematica ${ }^{\circledast}$ and RiskQ 4.0 functions used for calculations documented in Appendices 3.A-2.H.

Please note that more detailed explanation of Mathematica ${ }^{\circledast}$, RiskQ, and JUV analysis is beyond the scope of this report, and is provided in references cited. 


\section{Appendix 3.A}

\section{Concentration \\ of TCE @ BAFB (mg/L)}

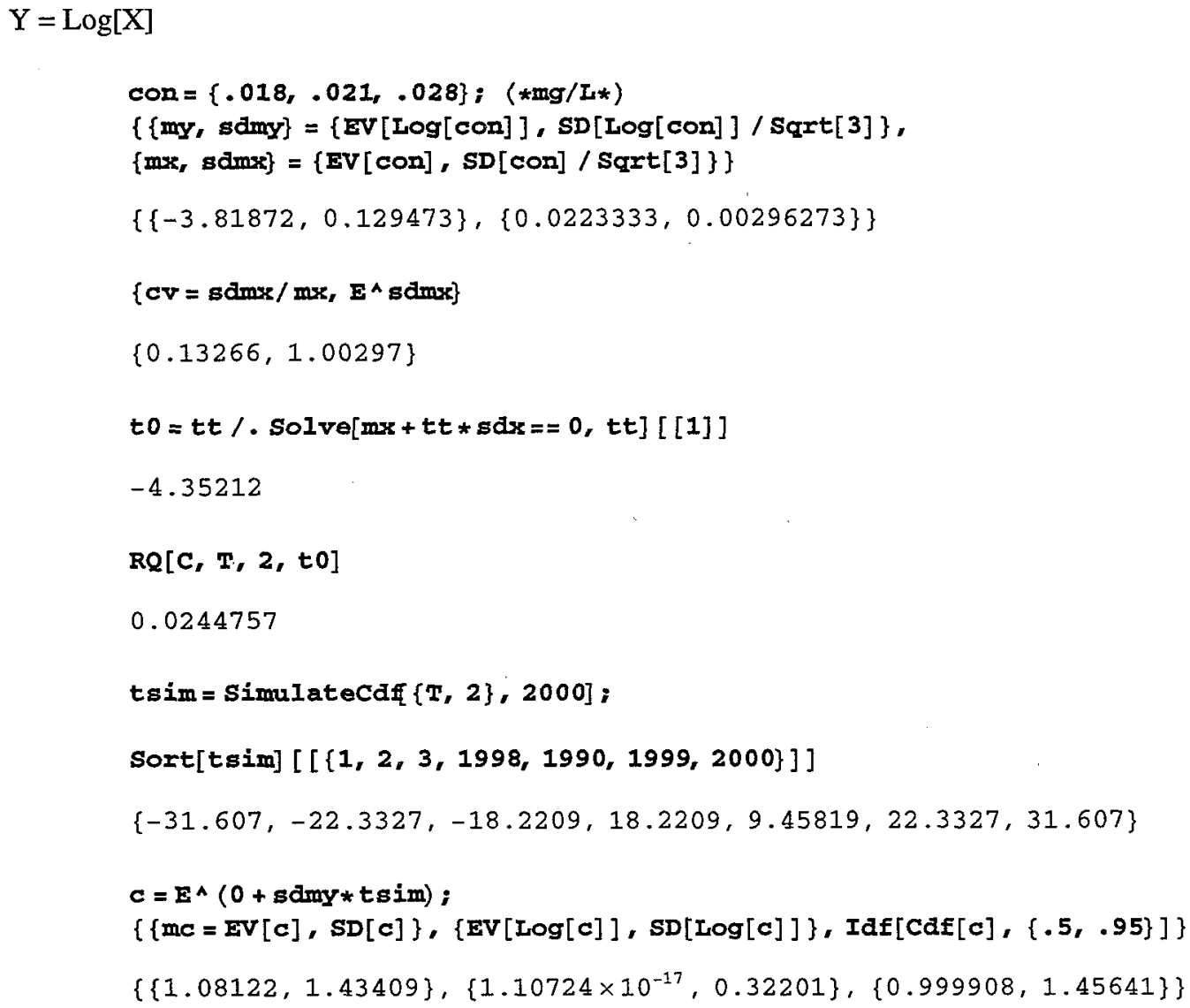

simulated conc. values

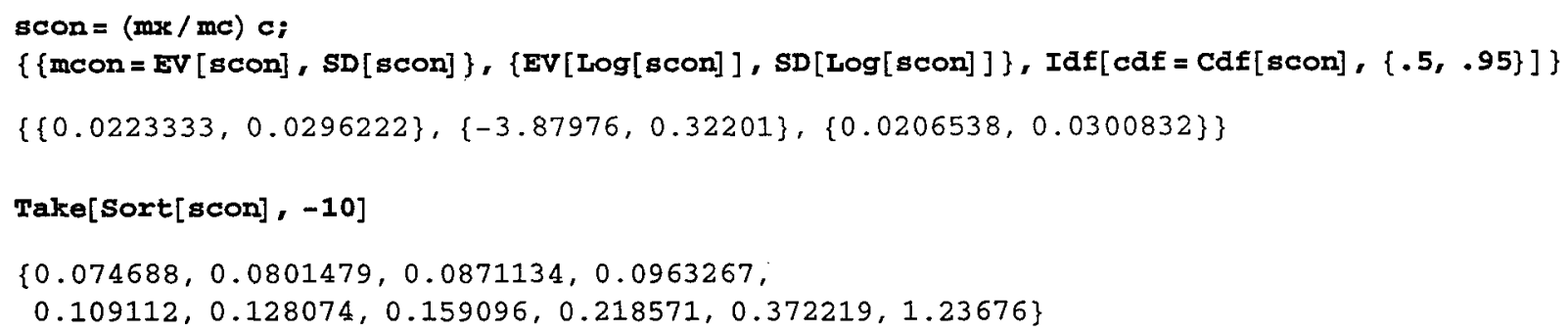


Plotcdf [cdf, Xmin $\rightarrow-.001, X \max ->.05]$;

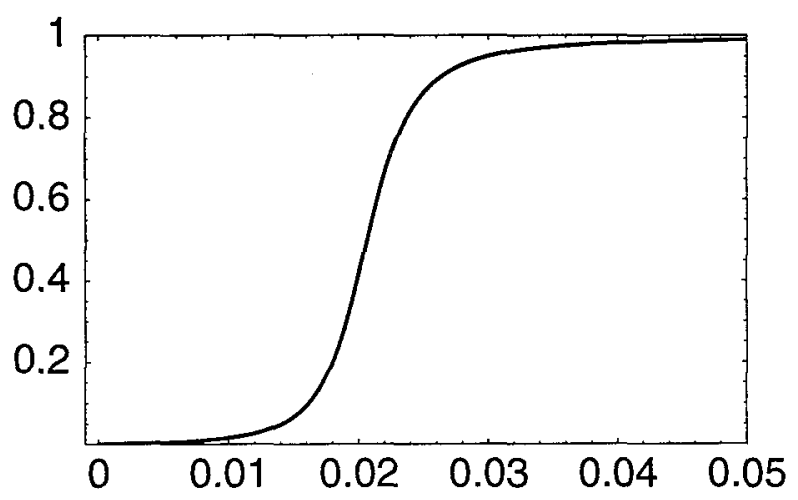

scdf = Standardizecdf $[$ cdf, 404];

WriteMatrix ["BogenHD: Desktop Folder:concentration.txt", N[scdf]];

- Log-Transform Utility Functions (where $X=\log Y$ )

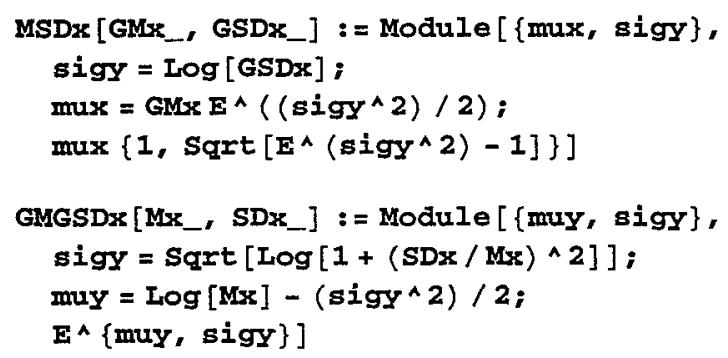

end 


\section{Appendix 3.B}

\section{Intakes}

2-17-99 (updated 4-26-99)

$<<$ RiskQ;

\section{- Log-Transform Utility Functions}

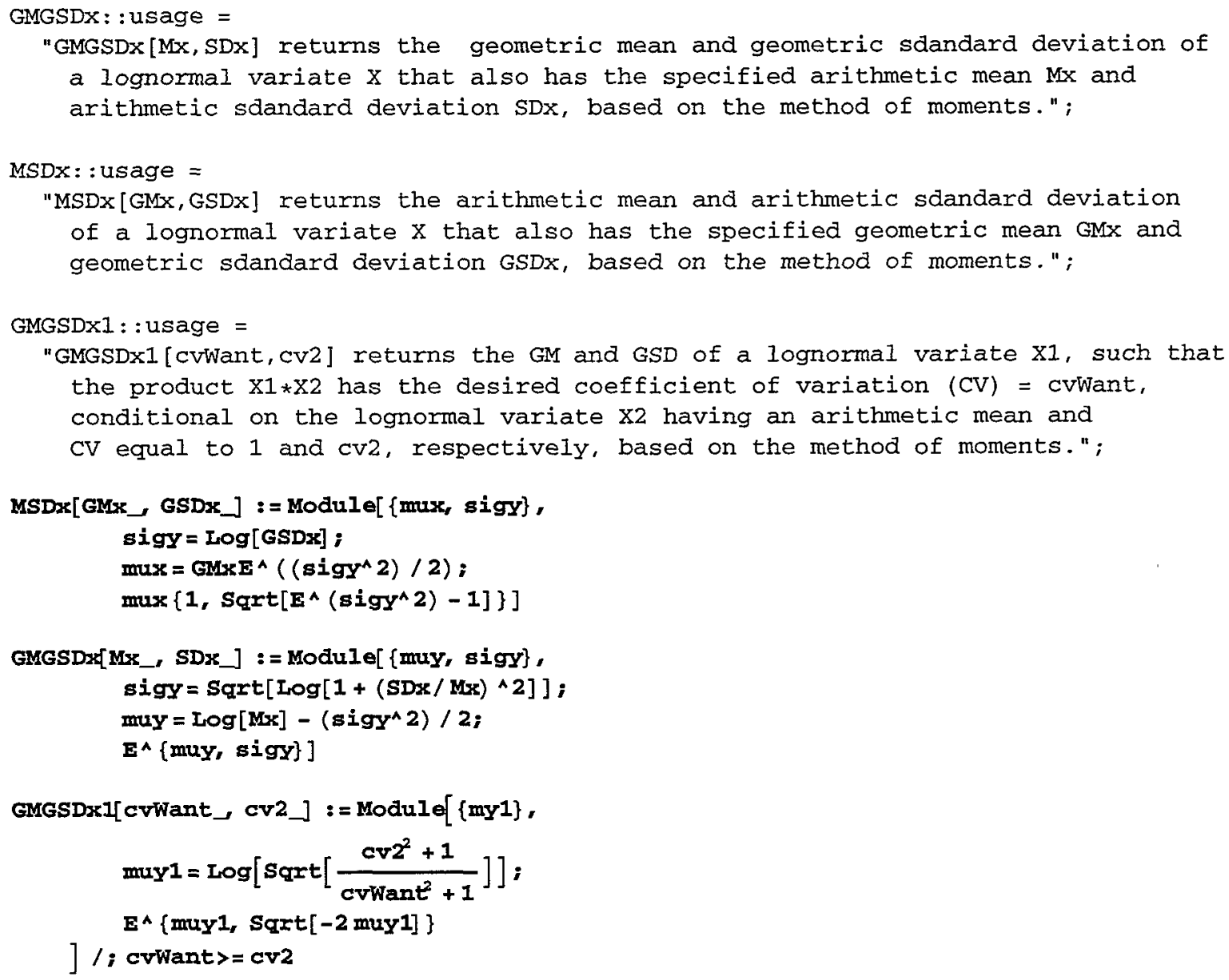


- Data on 1998 U.S. Population

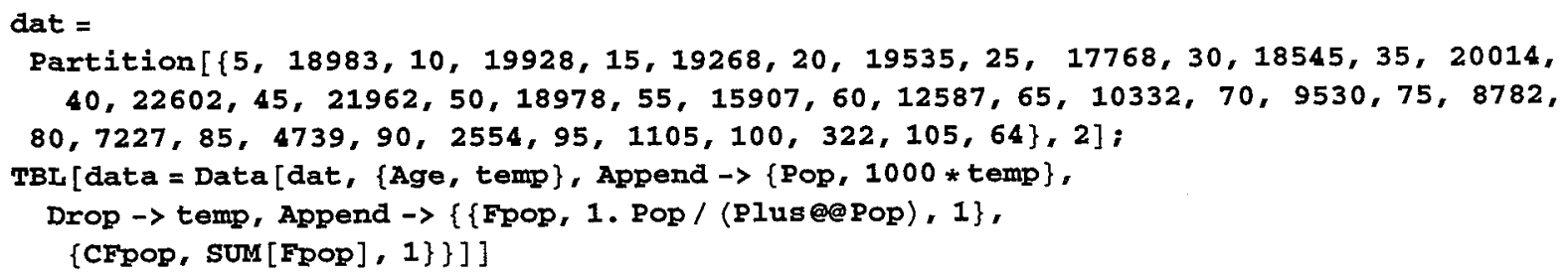

$\begin{array}{llll}\text { Age } & \text { Pop } & \text { Fpop } & \text { CFpop } \\ 5 & 18983000 & 0.0701173 & 0.0701173 \\ 10 & 19928000 & 0.0736078 & 0.143725 \\ 15 & 19268000 & 0.07117 & 0.214895 \\ 20 & 19535000 & 0.0721562 & 0.287051 \\ 25 & 17768000 & 0.0656295 & 0.352681 \\ 30 & 18545000 & 0.0684995 & 0.42118 \\ 35 & 20014000 & 0.0739255 & 0.495106 \\ 40 & 22602000 & 0.0834848 & 0.578591 \\ 45 & 21962000 & 0.0811208 & 0.659711 \\ 50 & 18978000 & 0.0700988 & 0.72981 \\ 55 & 15907000 & 0.0587555 & 0.788566 \\ 60 & 12587000 & 0.0464925 & 0.835058 \\ 65 & 10332000 & 0.0381632 & 0.873221 \\ 70 & 9530000 & 0.0352009 & 0.908422 \\ 75 & 8782000 & 0.032438 & 0.94086 \\ 80 & 7227000 & 0.0266943 & 0.967555 \\ 85 & 4739000 & 0.0175044 & 0.985059 \\ 90 & 2554000 & 0.00943368 & 0.994493 \\ 95 & 1105000 & 0.00408153 & 0.998574 \\ 100 & 322000 & 0.00118937 & 0.999764 \\ 105 & 64000 & 0.000236396 & 1 .\end{array}$

tpop = Plus@@Data $[$ data, Pop $]$

270732000

12 years and over: 200899000

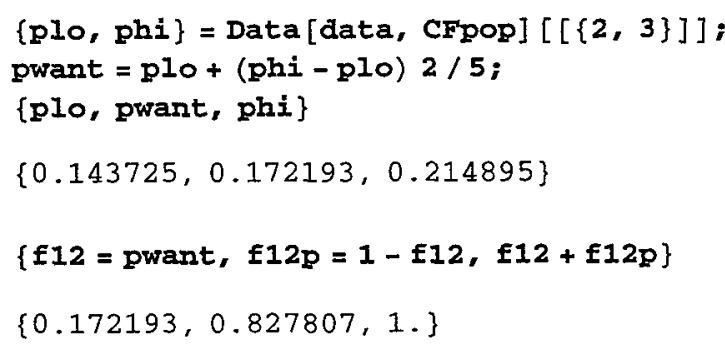

18 years and over: 200899000

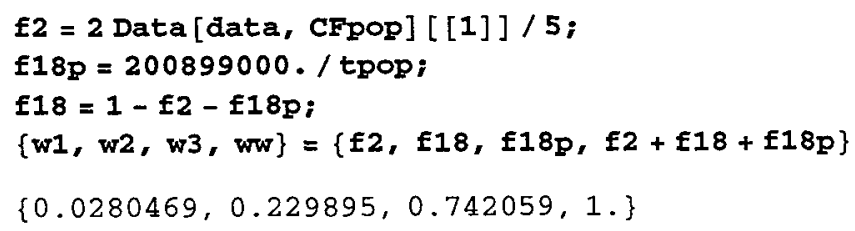




\section{- Ingestion (L/kg-d) Ershow and Cantor, 1989, Table 36 p. 76}

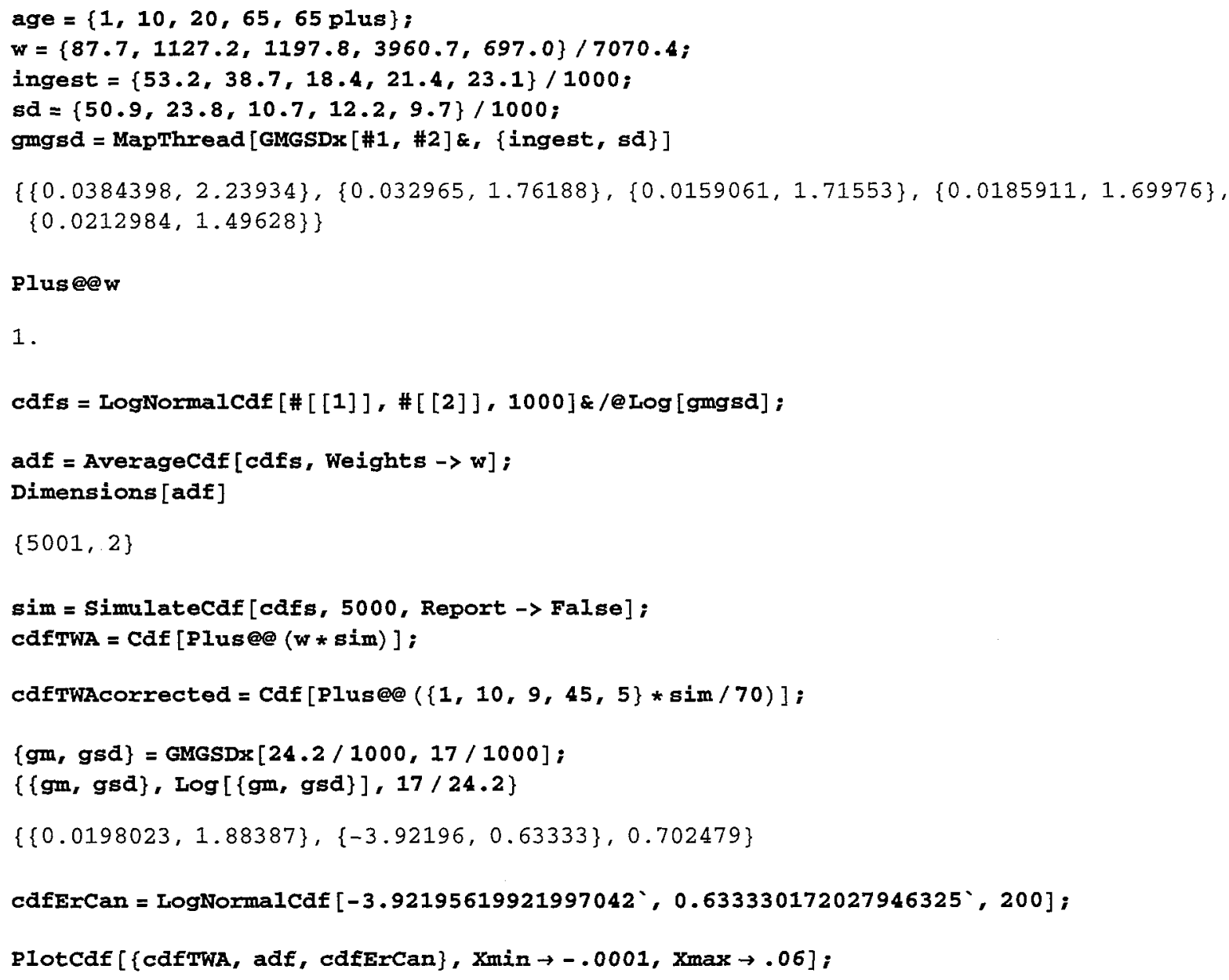


Plotcdf $[\{$ cdfTWA, cdfTWAcoxrected $\}, x \min \rightarrow-.0001, x \max \rightarrow .06]$;

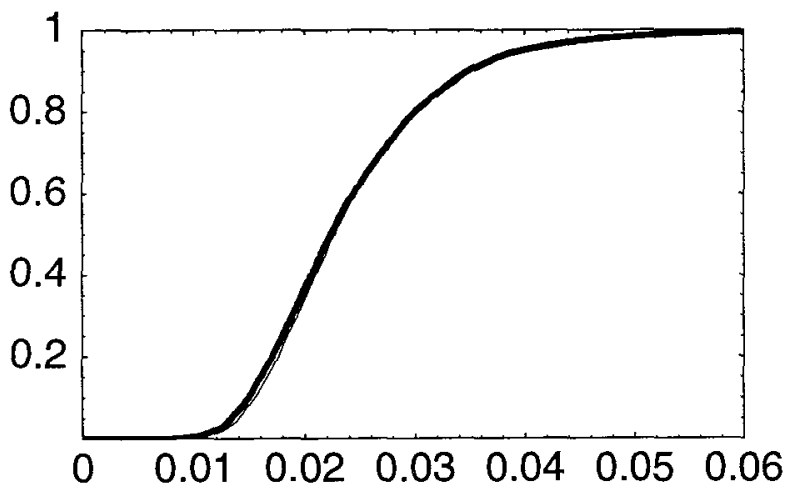

$\{$ EV [\#], SD [\#], Idf [\#, \{.5,.95\}]\}\&/@ \{cdfTWA, cdfTWAcorrected, adf, cdfErCan\}

$\{\{0.0241953,0.00802976,\{0.0227623,0.0390576\}\},\{0.0240441,0.00862608$,

$\{0.0223285,0.0398658\}\},\{0.024215,0.0170167,\{0.0200231,0.054078\}\}$,

$\{0.0242229,0.0170924,\{0.0198023,0.0561224\}\}\}$

end

\section{SABW Ratio calculations}

- Distribution of Body Surface Area to Body Weight (cm2/kg) Ratio (Phillips et al., 1993)
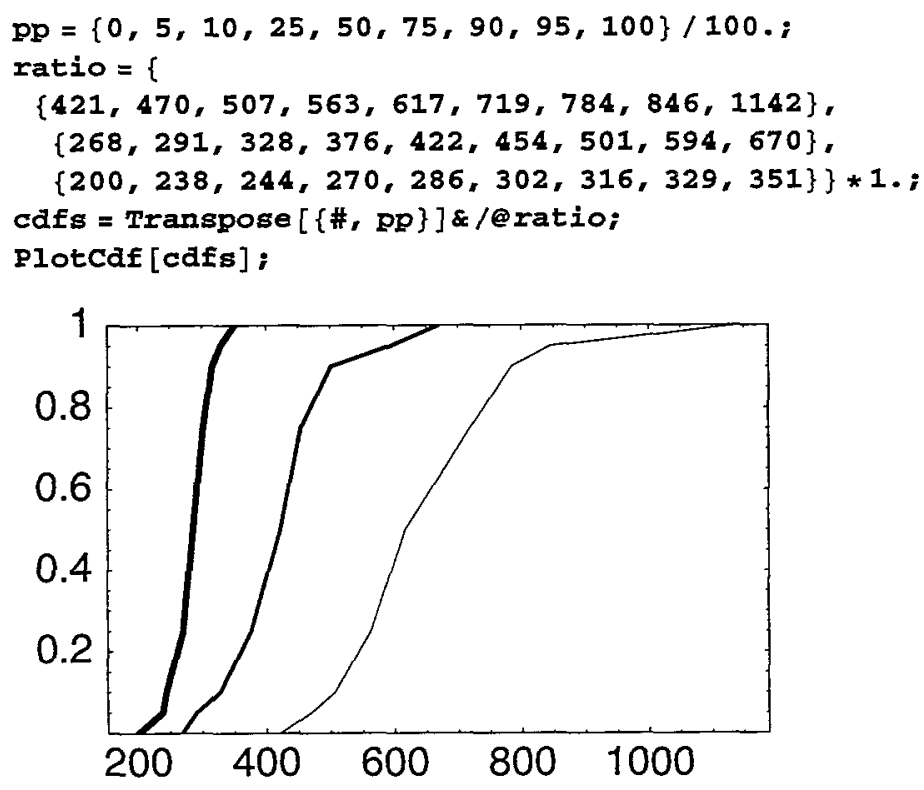


\section{- sabwALL}

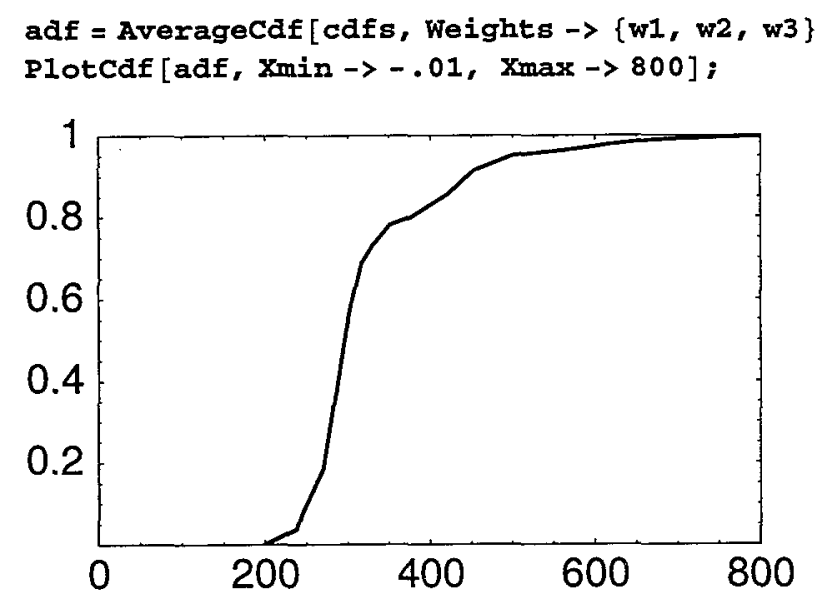

TBL [adf]

$\begin{array}{ll}200 . & 0 \\ 238 . & 0.0371029 \\ 244 . & 0.0742059 \\ 268 . & 0.176952 \\ 270 . & 0.186514 \\ 286 . & 0.380025 \\ 291 . & 0.440497 \\ 302 . & 0.571456 \\ 316 . & 0.687114 \\ 328 . & 0.725091 \\ 329 . & 0.728664 \\ 351 . & 0.781572 \\ 376 . & 0.799532 \\ 421 . & 0.855756 \\ 422 . & 0.857034 \\ 454 . & 0.915424 \\ 470 . & 0.927621 \\ 501 . & 0.951541 \\ 507 . & 0.95251 \\ 563 . & 0.963639 \\ 594 . & 0.971495 \\ 617 . & 0.97796 \\ 670 . & 0.98962 \\ 719 . & 0.992988 \\ 784 . & 0.997195 \\ 846 . & 0.998598 \\ 1142 . & 1 .\end{array}$

$\{$ EV $[$ adf $], \operatorname{Idf}[$ adf, .95] $\}$

$\{325.881,499.003\}$

WriteMatrix ["BogenHD: Desktop Folder: sabwratioALL.txt", adf];

$\{x, y\}=\operatorname{Transpose}[\operatorname{Rest}[\operatorname{Drop}[\operatorname{adf},-1]]]$

$\{\{238,244 \ldots 268 \ldots 270 \ldots, 286 \ldots 291,302 \ldots, 316 \ldots 328 \ldots 329 \ldots 351 \ldots 376 \ldots$,

$421 \ldots, 422,454 \ldots 470 \ldots, 501 \ldots 507 \ldots 563 \ldots 594 \ldots 617 \ldots 670 \ldots, 719 ., 784 \ldots 846$.$\} ,$

$\{0.0371029,0.0742059,0.176952,0.186514,0.380025,0.440497,0.571456,0.687114$,

$0.725091,0.728664,0.781572,0.799532,0.855756,0.857034,0.915424,0.927621$,

$0.951541,0.95251,0.963639,0.971495,0.97796,0.98962,0.992988,0.997195,0.998598\}\}$ 


\section{$x y=\operatorname{Transpose}[\{$ NormalCdf $[y$, Inv $], x\}]$}

$\{\{-1.78534,238\},.\{-1.44516,244\},.\{-0.927042,268$.$\} ,$ $\{-0.890815,270\},.\{-0.305415,286\},.\{-0.149709,291\},.\{0.180083,302$. $\{0.487687,316\},.\{0.598033,328\},.\{0.608776,329\},.\{0.777512,351$.$\} ,$ $\{0.839952,376\},.\{1.06145,421\},.\{1.06709,422\},.\{1.37493,454$.$\} ,$ $\{1.4583,470\},.\{1.65998,501\},.\{1.66969,507\},.\{1.79457,563\},.\{1.90324,594\}$, $\{2.01334,617\},.\{2.31232,670\},.\{2.45666,719\},.\{2.76978,784\},.\{2.98837,846\}$.

PlotData $[\mathbf{x y}]$;

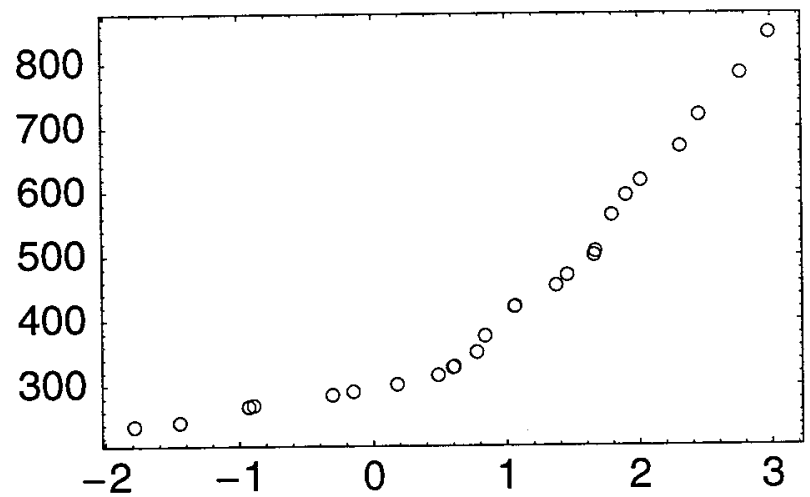

end

\section{- sabwTWA}

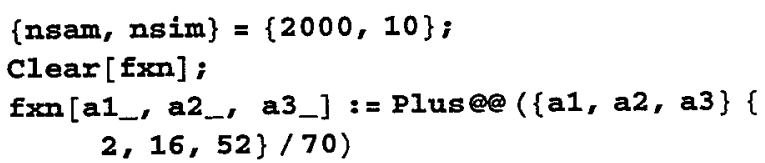




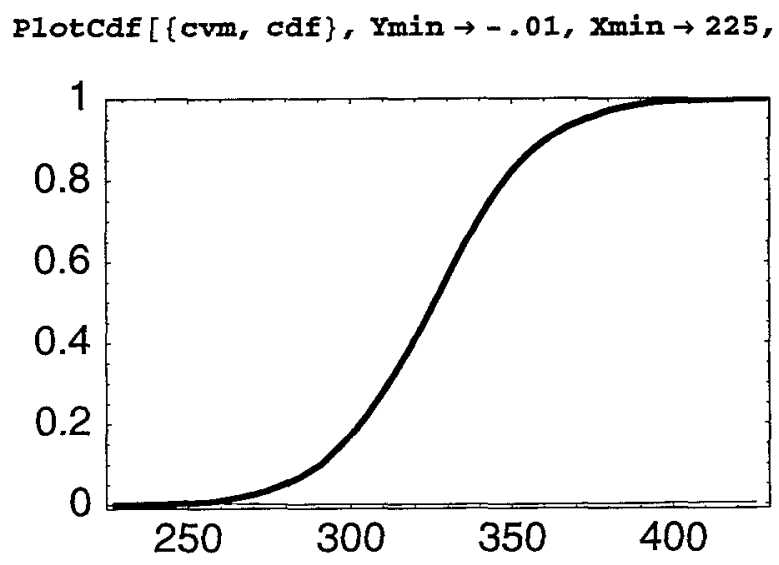

Sqrt [777.660703210145509]

27.8865685090537002

$\{$ EV [cdf, Empirical $\rightarrow$ True] $, \operatorname{SD}[c d f$, Empirical $\rightarrow$ True $], \operatorname{Idf}[c d f,\{.5, .95\}]\}$

$\{325.884,27.8746,\{326.046,373.11\}\}$

sdf $=$ Standardizecdf $[$ cdf, 404];

$\{E V[s d f$, Empirical $\rightarrow$ True $], \operatorname{Idf}[s d f,\{.5, .95\}]\}$

$\{325.875,\{326.046,373.235\}\}$

WriteMatrix ["Bogen's:Desktop Folder:sabwratioTWA.txt", sdf];

$\{x, y\}=$ Transpose $[\operatorname{Rest}[\operatorname{Drop}[$ cdf, -1$]]]$;

$\mathbf{x y}=$ Transpose $[\{$ NormalCdf $[y, \operatorname{Inv}], \mathbf{x}\}] ;$

fit $=$ FIT $[x y,\{1, X\}, X$, Report $\rightarrow$ True $]$;

$\begin{array}{lllll}\text { Coef } & \text { LS Est. } & \text { SD } & 958 \text { LCL } & 95 \% \text { UCL } \\ \text { q[0] } & 325.884 & 0.0240524 & 325.837 & 325.931 \\ \text { q[1] } & 27.9467 & 0.0241325 & 27.8994 & 27.994\end{array}$

$\mathrm{R}^{2}=0.998512$

$F(1,1998)=1.34108 \times 10^{6} \quad 2$-tail $\mathrm{p}=3.7487211392 \times 10^{-2827}$

PlotData [xy, FitTo $\rightarrow\{$ fit $.\{1, x\}, x\}]$;

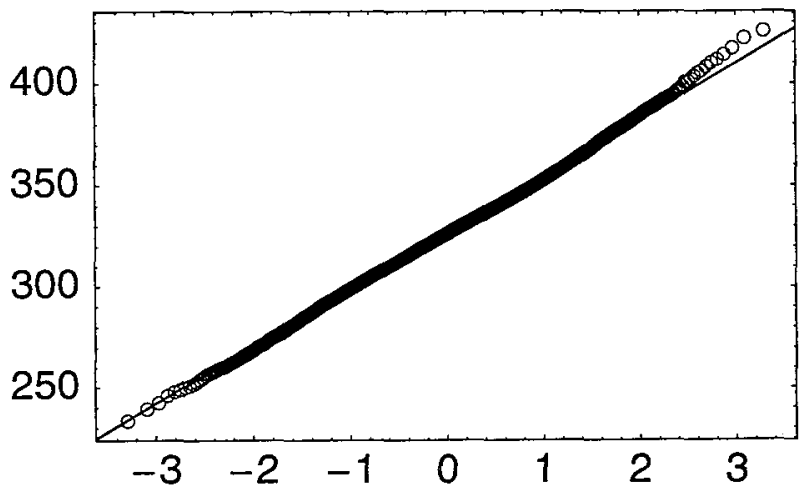




\section{- Inhalation (L/kg-d) OHEA. 1996. Stochastic Analysis, p. 3-31 - 3-32.}

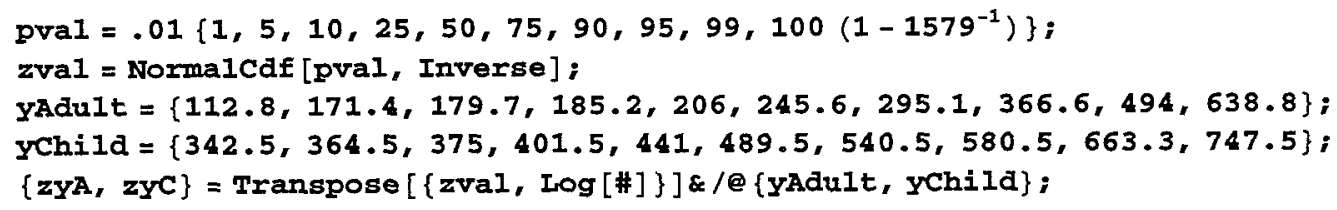

Option 1 (Not Used): Calculate lognormal parameters from OHEA data

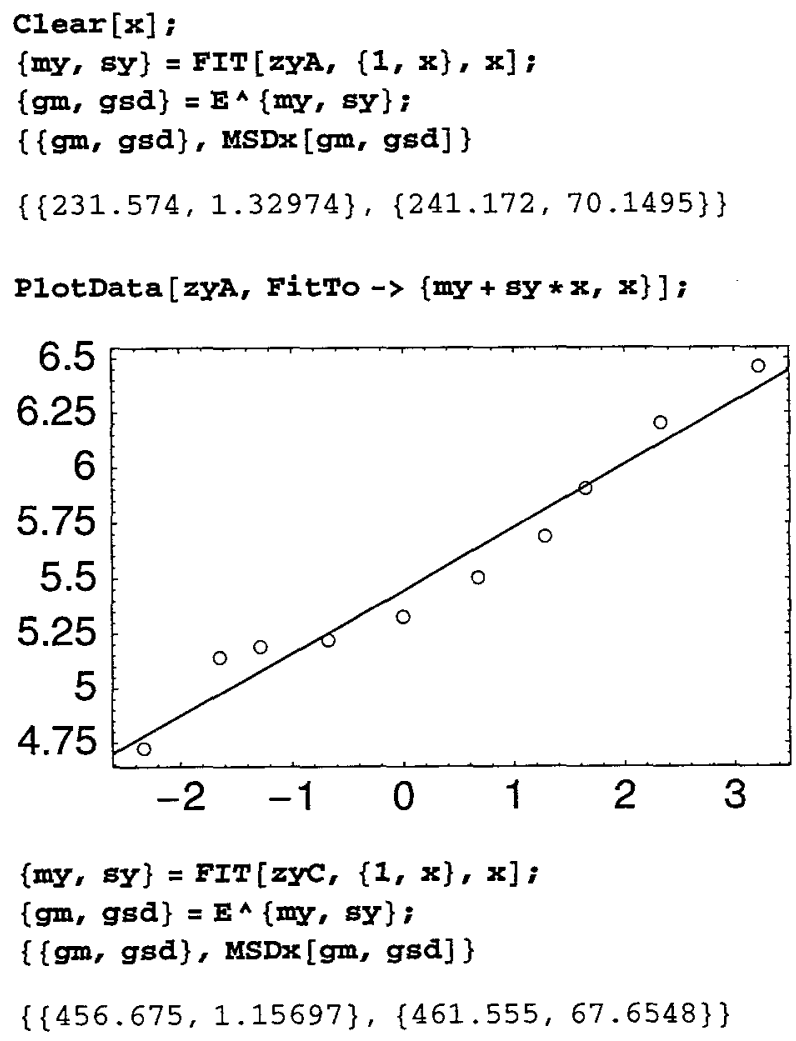




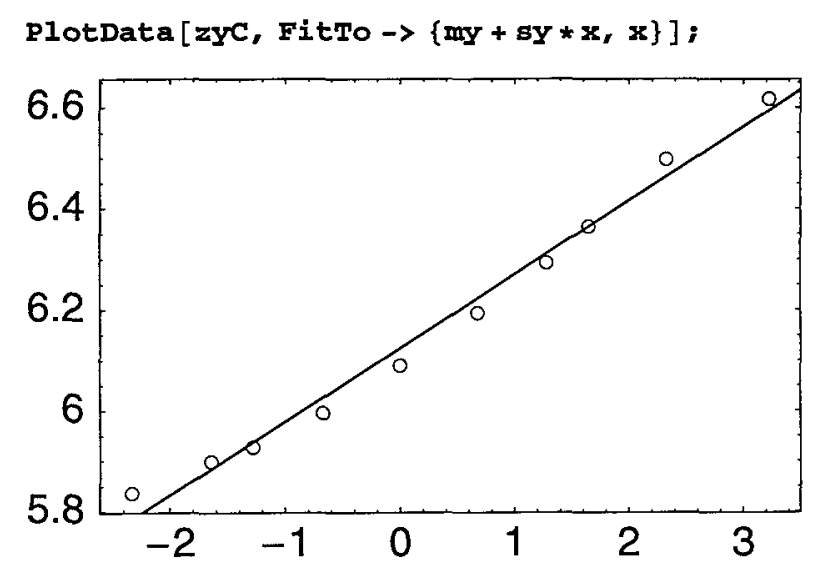

Option2 (Used): Use lognormal parameters derived from reported OHEA mean and SD values for Adult and Child distributions

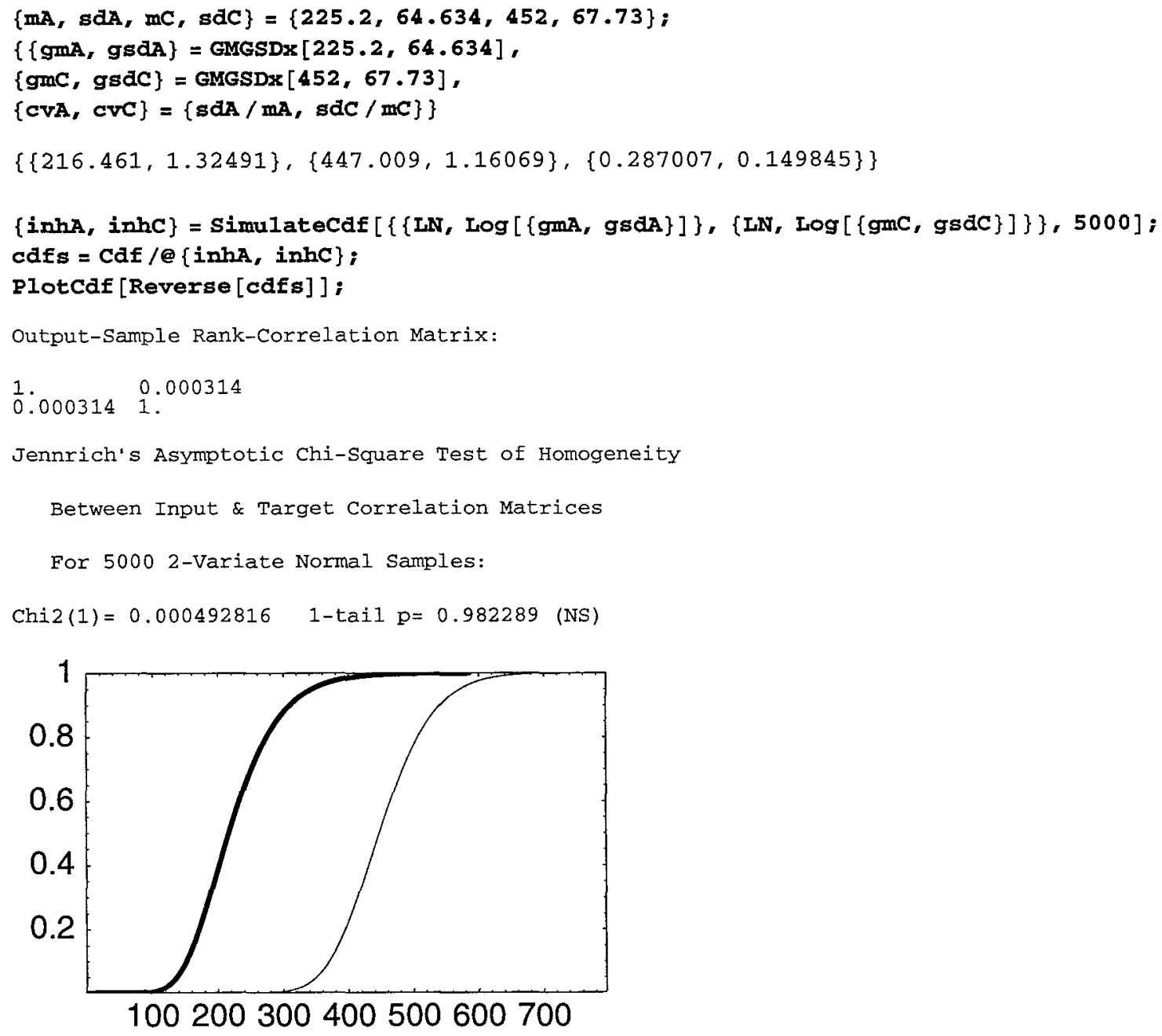

100200300400500600700

From Finley et al. 1994 (CalEPA 1996, p. 10-7), the BW distribution for adult males \& females is $\sim \mathrm{LN}$ and CV[BW] $=$ $\sim 0.22$ : 


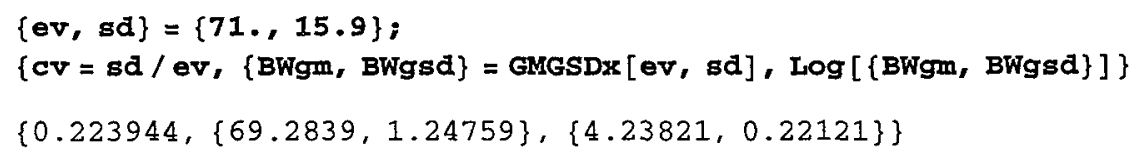

From CalEPA/OHEA (1996, Stochastic Analysis, p. 3-31 - 3-32; cit. above), cvA = CV[24*Q/BW] $=\mathrm{CV}[\mathrm{Qtot} / \mathrm{BW}]=\sim$ 0.3 , where $\mathrm{Q}$ denotes total ventilation rate in $\mathrm{L} / \mathrm{h}$. From Allen and Fisher (1993), alveolar ventilation rate in $\mathrm{L} / \mathrm{h}$ is modeled as $\mathrm{Q} \sim 12.9^{*} \mathrm{BW} \mathrm{W}^{\wedge} 0.7$, and Qtot $\sim \mathrm{kQ}$ for some constant $\mathrm{k}$. Now let VQ be $\mathrm{LN}$-distributed with an arithmetic mean of 1 , where VQ represents variation in $\mathrm{Q}$ not attributable to that in $\mathrm{BW}$. Thus, $\mathrm{Q} \sim 12.9 * \mathrm{VQa} * \mathrm{BW} \wedge .7$, whence $\mathrm{Q} / \mathrm{BW} \sim$ $12.9 * \mathrm{VQ}^{*} \mathrm{BW} \mathrm{W}^{\wedge}-0.3$. It follows from the method of moments that $\mathrm{CV}\left[\mathrm{BW}{ }^{\wedge}-.3\right]=\mathrm{CV}\left[\mathrm{BW}^{\wedge} .3\right]=0.06644$, whence $\mathrm{CV}[\mathrm{VQ}]=$ $0.2919, \mathrm{GM}[\mathrm{VQ}]=0.9599, \mathrm{GSD}[\mathrm{VQ}]=1.331, \log [\{\mathrm{GM}[\mathrm{VQ}], \mathrm{GSD}[\mathrm{VQ}]\}]=\{-0.0408868,0.285961\}$.

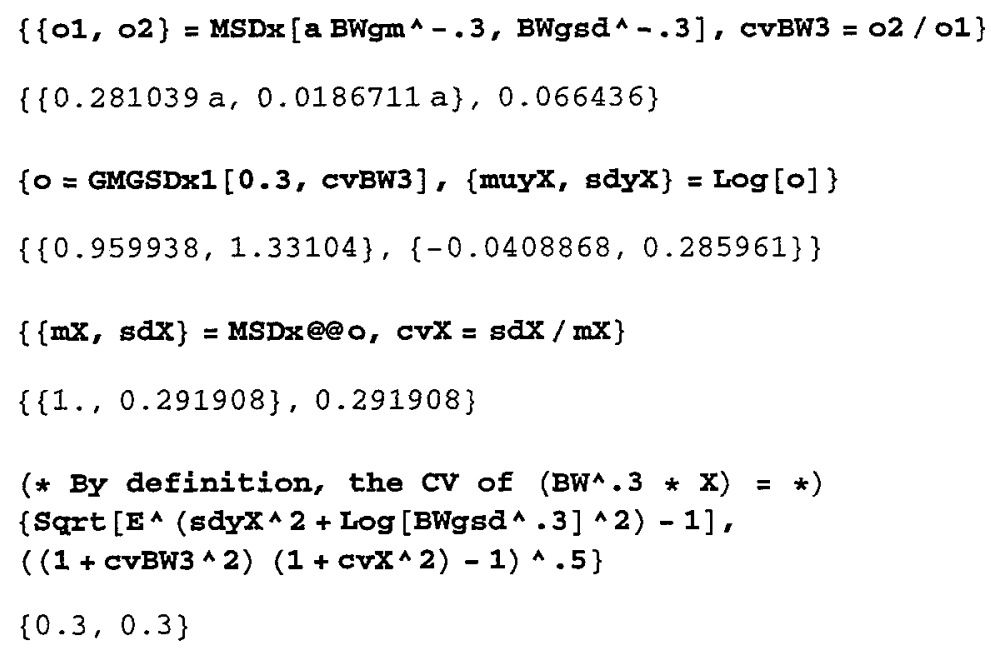

\section{- InhaleALL}

$\{f 12, f 12 p\}$

$\{0.172193,0.827807\}$

adf = AverageCdf $[$ cdfs, Weights $\rightarrow\{f 12 p, f 12\}]$; sadf $=\mathbf{N}[$ Standardizecdf $[\operatorname{adf}, 404]]$;

Plotcdf [sadf, $X \min \rightarrow-.001, Y \min \rightarrow-.001]$;

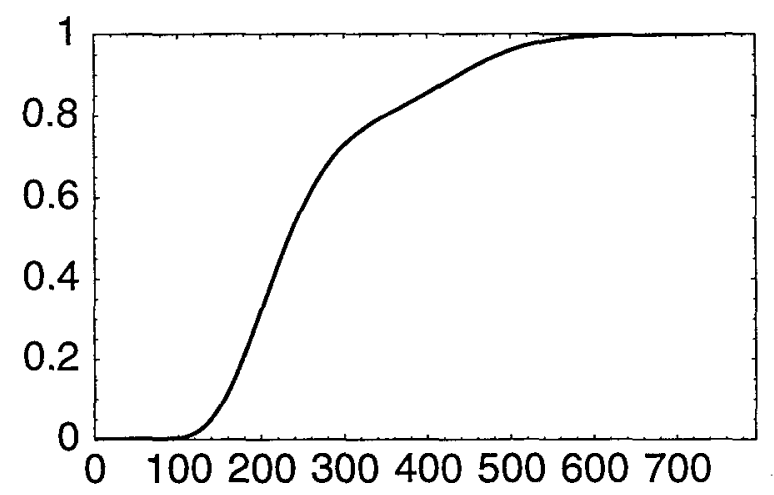


$\{E V[\#], \operatorname{SD}[\#], \operatorname{Idf}[\#,\{.5, .95\}]\} \& / @\{\operatorname{adf}, \operatorname{sadf}\}$

$\{\{264.165,107.491,\{233.106,487.138\}\},\{264.165,108.051,\{233.106,487.146\}\}\}$

$N[\{\{p A, p C\}=\operatorname{Edf}[\#, 487.138] \& / @ c d f s, w p=\{£ 12 p * p A, f 12 * p C\}, P 1 u s @ e w p\}, 10]$

$\{\{0.9982290631,0.7181436766\},\{0.8263408411,0.1236594327\}, 0.9500002739\}$

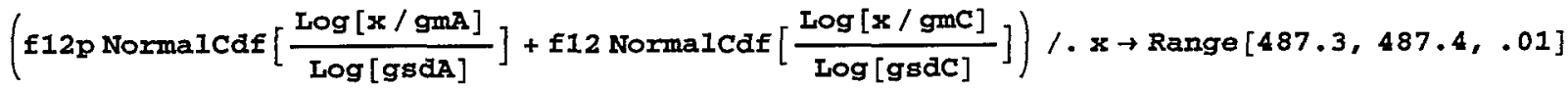

$\{0.949947,0.949956,0.949964,0.949972,0.949981,0.949989,0.949997,0.950006$,

$0.950014,0.950023\}$

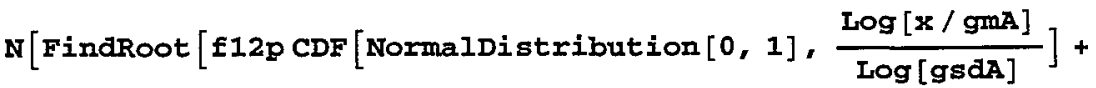

£12 CDF $\left[\right.$ Norma1Distribution $\left.\left.[0,1], \frac{\log [x / g m C]}{\log [g s d C]}\right]==95 / 100,\{x, 485,480,490\}\right]$,

$16]$

$\{x \rightarrow 487.3630111243049\}$

WriteMatrix ["Bogenfo: Desktop Folder: inhaleALL.txt", sadf] ;

\section{- InhaleTWA}

$\{\{f 12 p, f 12\},\{f 12 p, f 12\} 70\}$

$\{\{0.827807,0.172193\},\{57.9465,12.0535\}\}$

$\{$ nsam, nsim $\}=\{2000,10\} ;$

clear [fxen];

fxn $\left[a 1_{-}, a 2_{2}\right]:=$ PIusee $(\{a 1, a 2\}\{$

$58,12\} / 70)$

Timing $[\{j e n, c d f, c v m\}=$ QUAnalyze [cdfs, fxm, nsam, nsim] i]

$\{143.533$ Second, Null $\}$

TBL/@jen

$\left\{\begin{array}{lllll}\text { Mean }[\Delta r] & \text { Max }[|\Delta r|] & \text { JennrichChi2 } & \text { DegFr } & \text { Pval } \\ 0.0010747 & 0.00945733 & 0.178882 & 1 & 0.672335\end{array}\right.$

Fractile Value CVM( 8$)$

$\begin{array}{lll}0.01 & 166.336 & 0.275487\end{array}$

$\begin{array}{lll}0.05 & 187.965 & 0.14204\end{array}$

$\begin{array}{lll}0.5 & 257.154 & 0.0674972\end{array}$

$\begin{array}{lll}0.95 & 362.928 & 0.17087\end{array}$

$\begin{array}{lll}0.99 & 424.294 & 0.272136\end{array}$

Mean $263.8657 .46556 \times 10^{-7}$

Variance $2937.4 \quad 0.0463849$ 
Plotcdf $[\{c v m$, cdf $\}, Y \min \rightarrow-.01, X \min \rightarrow 95, X \max \rightarrow 430]$;

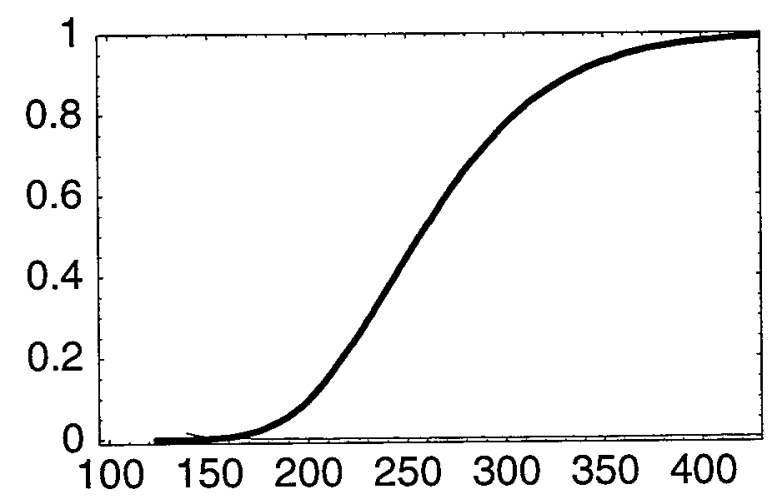

$\{$ EV [cdf, Empirical $\rightarrow$ True], $\operatorname{SD}[c d f$, Empirical $\rightarrow$ True], Idf [cdf, $\{.5, .95\}]\}$

$\{263.865,54.1887,\{257.154,362.928\}\}$

sdf = Standardizecdf $[\mathrm{cdf}, 404]$;

\{EV[sdf, Empirical $\rightarrow$ True], SD[sdf, Empirical $\rightarrow$ True], Idf [sdf, \{.5, .95\}]\}

$\{263.752,53.5784,\{257.154,362.847\}\}$

WriteMatrix["Bogen's:Des]top Folder:inhaleTwA.txt", sdf]; 


\section{Appendix 3.C}

\section{Fraction of Lifetime at One Local Residence}

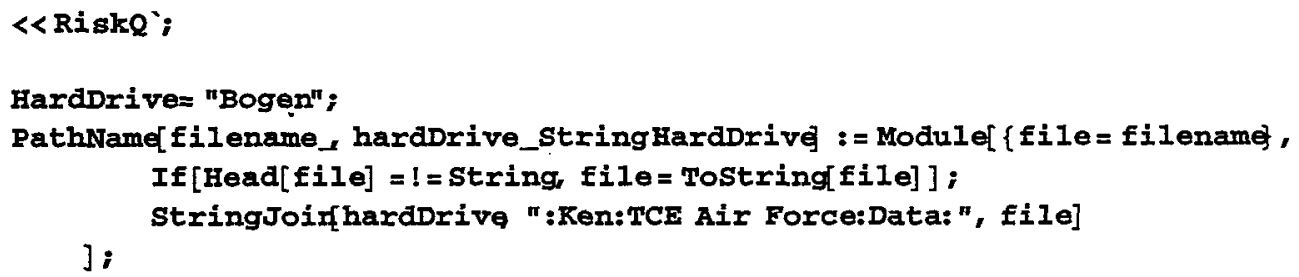

Israeli, M., and C. Nelson. 1992. Distribution and expected time of residence for U.S. housholds. Risk Anal. 12, 65-72.

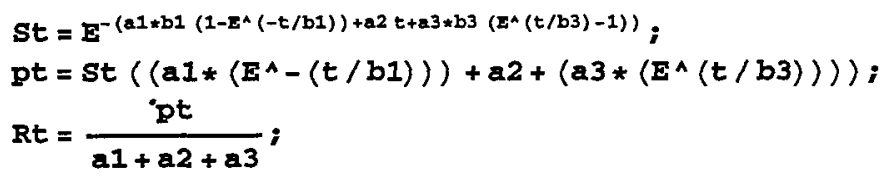

Coefficients $\mathrm{a}$ and $\mathrm{b}$ all have units of $y^{-1}$ and $\mathrm{y}$, respectively, from Israeli and Nelson Table II (All households, W-Rgn)

- All households

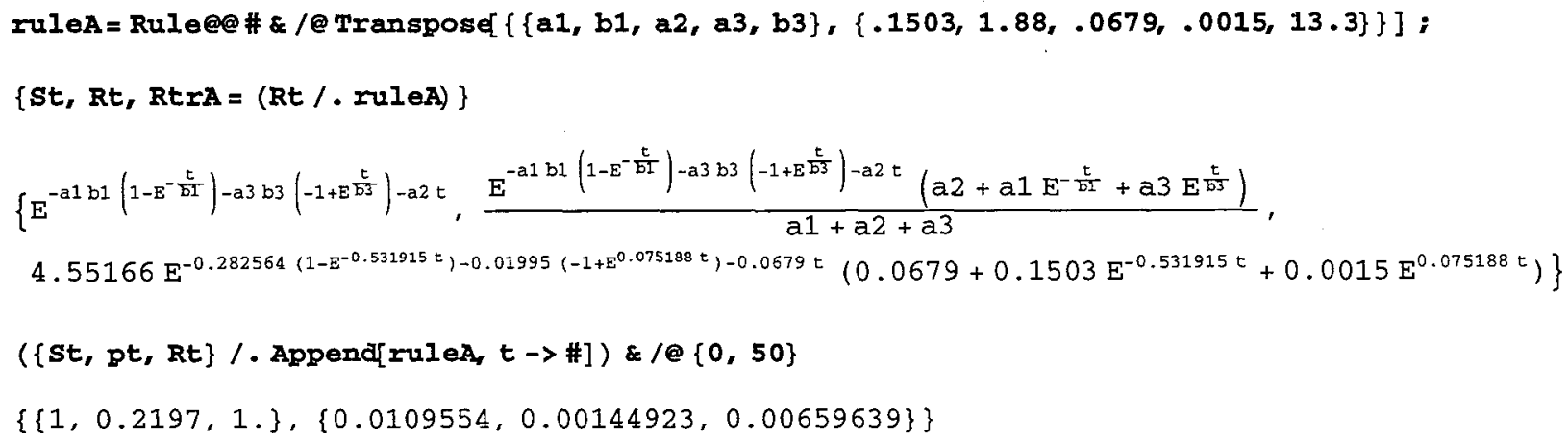




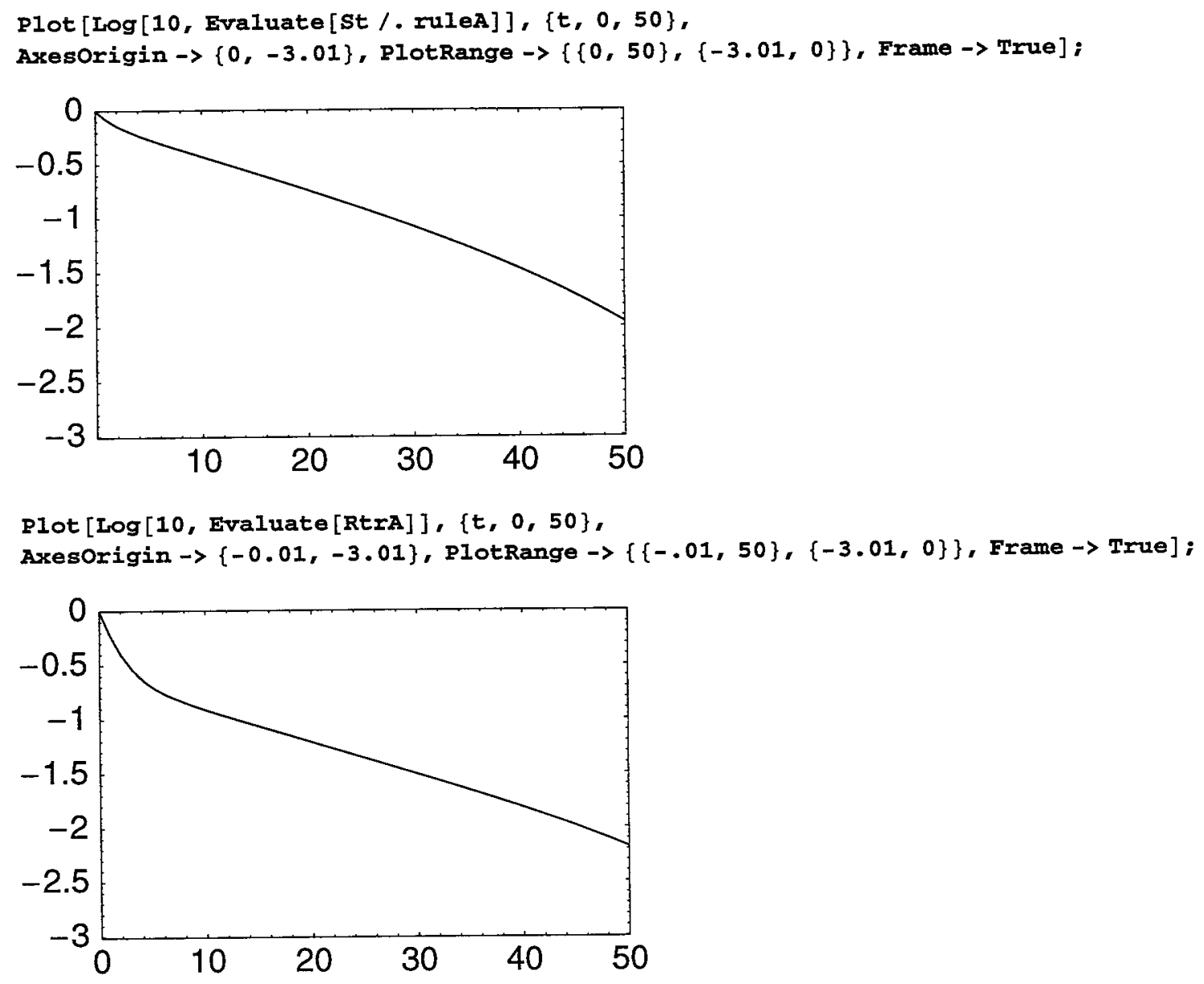

\section{- Western Region}

ruleW $=\operatorname{Rule@@\& ~} / @ \operatorname{Transpose}[\{\{a 1, b 1, a 2, a 3, b 3\},\{.2029,1.74, .0832, .0008,10.3\}\}]$

$\{\mathrm{a} 1 \rightarrow 0.2029, \mathrm{~b} 1 \rightarrow 1.74, \mathrm{a} 2 \rightarrow 0.0832, \mathrm{a} 3 \rightarrow 0.0008, \mathrm{~b} 3 \rightarrow 10.3\}$

RtrW $=($ Rt $/$. ruleW $)$

$3.48554 E^{-0.353046\left(1-E^{-0.574713 t}\right)-0.00824\left(-1+E^{0.0970874 t}\right)-0.0832 t}\left(0.0832+0.2029 E^{-0.574713 t}+0.0008 E^{0.0970874 t}\right)$

( $(S t$, pt, Rt $\} /$. Append $[r u l e W, t \rightarrow \#]) \& / @\{0,50\}$

$\{\{1,0.2869,1\},.\{0.0038411,0.00071383,0.00248808\}\}$ 


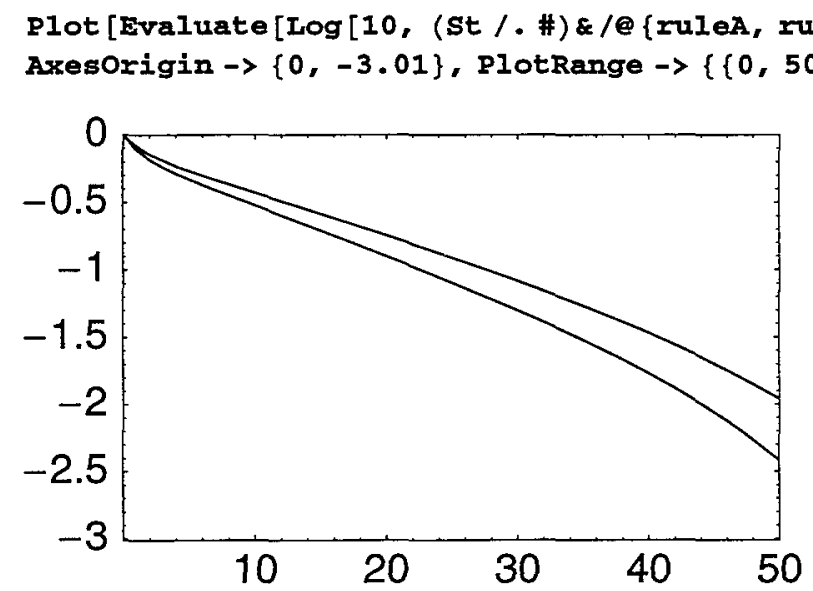

Plot [Evaluate $[$ Log $[10,\{$ RtrA, RtrW $\}]],\{t, 0,50\}$,

AxesOrigin $\rightarrow\{-0.01,-3.01\}$, PlotRange $\rightarrow\{\{-.01,50\},\{-3.01,0\}\}$, Frame $\rightarrow$ True $]$;

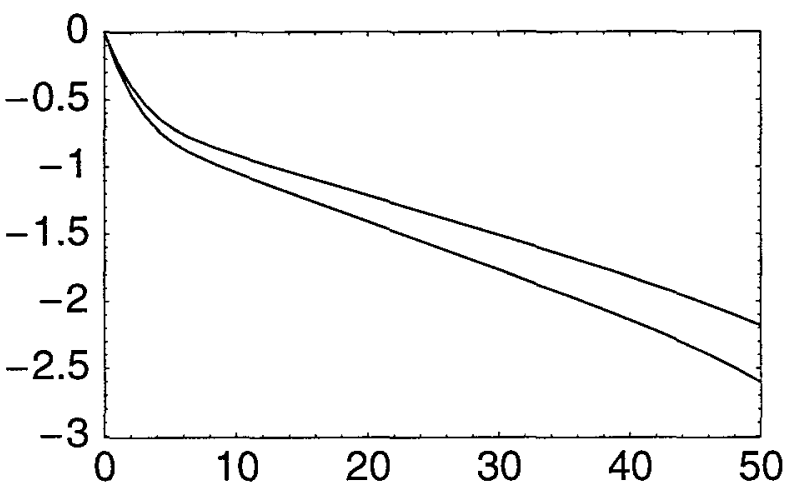

Adaptation of model to account for fraction Fm of moves that are out of a Western-region water distribution system

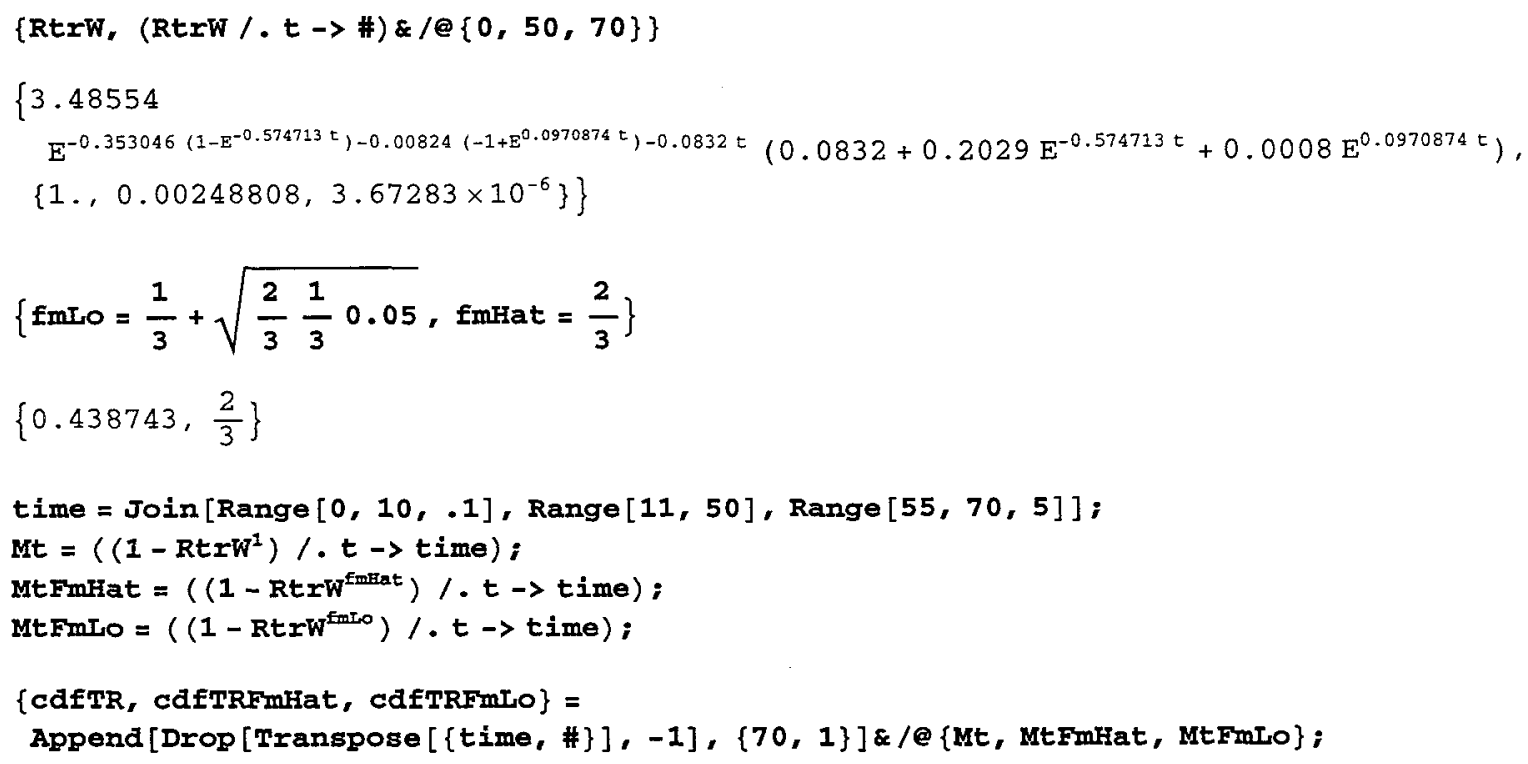


RtrW /. t $\rightarrow 1$

0.544555

PlotData [\{cdfTRFmLo, cdfTRFmHat, cdfTR $\}, \mathrm{Xmin} \rightarrow-.01, \mathrm{Xmax} \rightarrow>70, \mathrm{Ymin} \rightarrow \mathbf{0}, \mathrm{Ymax} \rightarrow>1$, Dotsize $\rightarrow\{.0001, .008, .0125\}$, style $\rightarrow 0$, JoinPoints $\rightarrow$ True, FitTo $\rightarrow\{$ RtrW, $t\}]$;

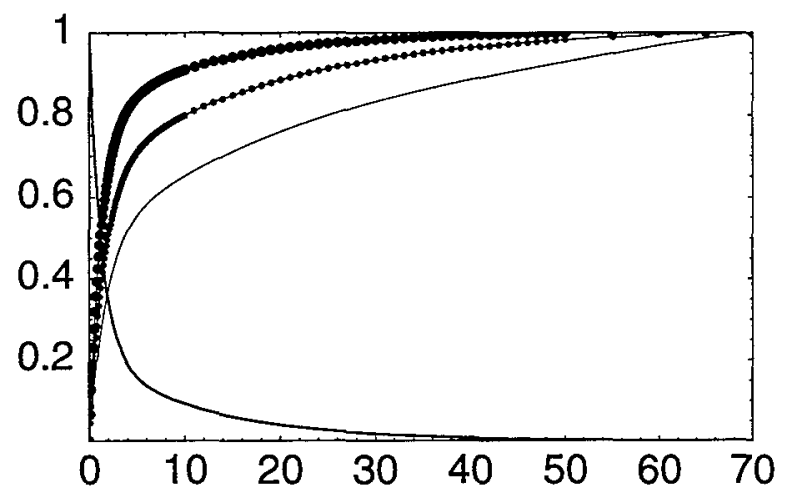

$\{E V[\operatorname{cdfTR}], \operatorname{Sqrt}[\operatorname{Var}[\operatorname{cdfTR}]], \operatorname{Idf}[\operatorname{cdfTR},\{.025, .5, .95, .975\}]\}$

$\{3.48741,6.83815,\{0.0378193,1.16603,17.0596,25.4481\}\}$

$\{\mathrm{EV}[$ cdfTRFmHat $], \operatorname{sgrt}[\operatorname{Var}[$ cdfTRFmHat $]], \operatorname{Idf}[$ cdfTRFmHat, $\{.025, .5, .95, .975\}]\}$

$\{7.02862,11.8589,\{0.0560921,1.9295,35.0626,46.1341\}\}$

$\{$ EV [cdfTRFmLo], Sqxt [Var [cdfTRFmLo] ], Idf [cdfTRFmLo, $\{.025, .5, .95, .975\}]\}$

$\{12.9946,17.7241,\{0.0845741,3.63964,55.2843,61.7931\}\}$

Rt $=($ RtrW /. t $\rightarrow$ time);

$x y=$ Append $[$ Drop $[$ Transpose $[\{t i m e, R t\}],-1],\{70,0\}]$;

IRt $=$ Interpolation $[x y$, Interpolationorder $\rightarrow 1]$;

10.5/16. (* = US fraction moving to same county *)

0.65625

fms $=\operatorname{SimulateCdf}[\{\operatorname{Tri},\{1 / 3,2 / 3,1\}\}, 2000] ;$

Idf [Caf [fms], .05]

0.438716 
PlotCdf [Cdf [fms] ] ;

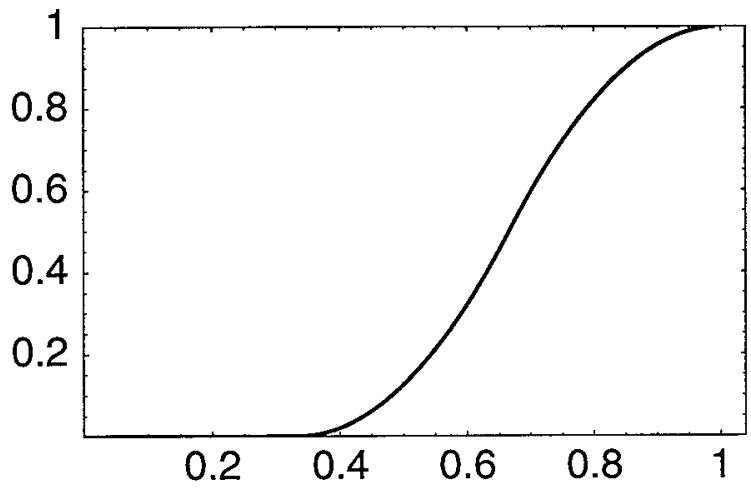

tRange $=$ Prepend $[$ time, $t]$

Tbar $=$ NIntegrate $[$ IRt $[t] \wedge \#$, Evaluate $[$ tRange $]] \& / @$ fms;

cdfTbar $=\operatorname{cdf}[$ Tbar $]$;

Plotcdf [cdfTbar, Xmin $\rightarrow-.01$, Xmax $\rightarrow 16]$;

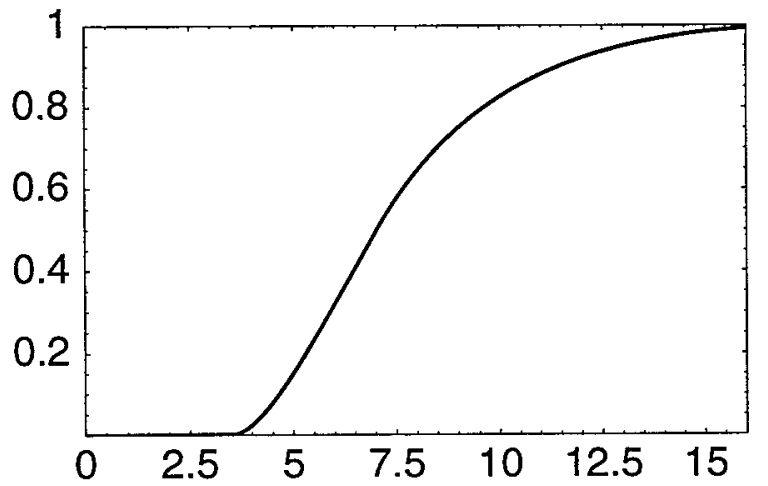

$\{$ EV $[$ Tbar $], \operatorname{Sqrt}[\operatorname{Var}[$ Tbar $]], \operatorname{Idf}[$ cdfTbar, $\{.025, .5, .975\}]\}$

$\{7.5984,2.67068,\{4.00903,7.03101,14.2792\}\}$

$D\left[a^{\wedge} \mathrm{Fm},\{\mathrm{Fm}, 2\}\right]$

$a^{\mathrm{Fm}} \log [a]^{2}$

cdfFm = Triangularcdf $[1 / 3,2 / 3,1,500]$ $\{$ EV $[\mathrm{CdfFm}], \operatorname{Var}[\mathrm{CdfFm}]\}$

$\{0.666667,0.0185294\}$

cdfFm = TriangularCdf $[1 / 3,2 / 3,1,10000]$; $\{\mathrm{EV}[\mathrm{cdfFm}], \operatorname{Var}[\operatorname{cdfFm}]\}$

$\{0.666667,0.0185186\}$ 


\section{$0.0185186464965580999^{\wedge}-1$}

53.999626818615568

Rti = Transpose $[x y][[2]]$;

fmbar $=2 / 3$;

fmVar $=1 / 54$;

$p i=1-\left((\operatorname{Rti} \wedge \operatorname{fmBar})\left(1+\frac{\log [R t i]^{2}}{2} \operatorname{fmVar}\right)\right)$

pi = Append $[\operatorname{Drop}[\mathrm{pi},-1], 1]$;

cdfTangbr $=$ Transpose $[\{$ time, pi $\}]$;

PlotCdf [cdfTangbr, $\mathrm{Xmin} \rightarrow-.01, \mathrm{Xmax}->70]$;

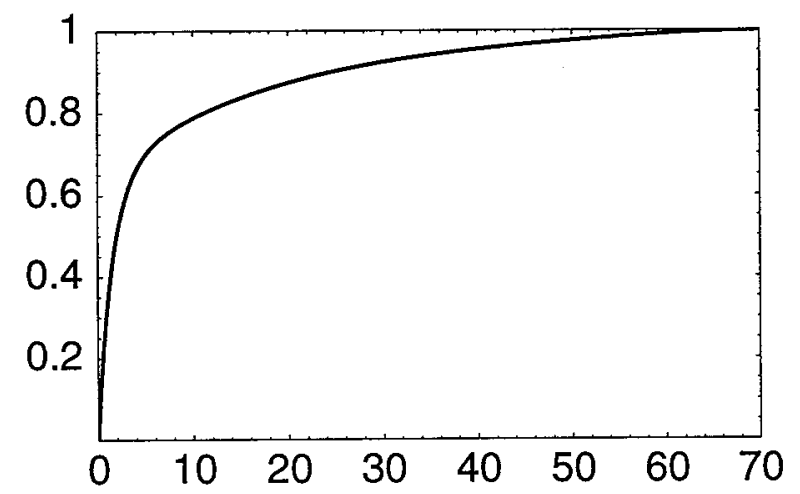

PlotCdf $[$ cdfTangbr, cdfTbar $\}, x \min \rightarrow-.01, \mathrm{Xmax} \rightarrow>70]$;

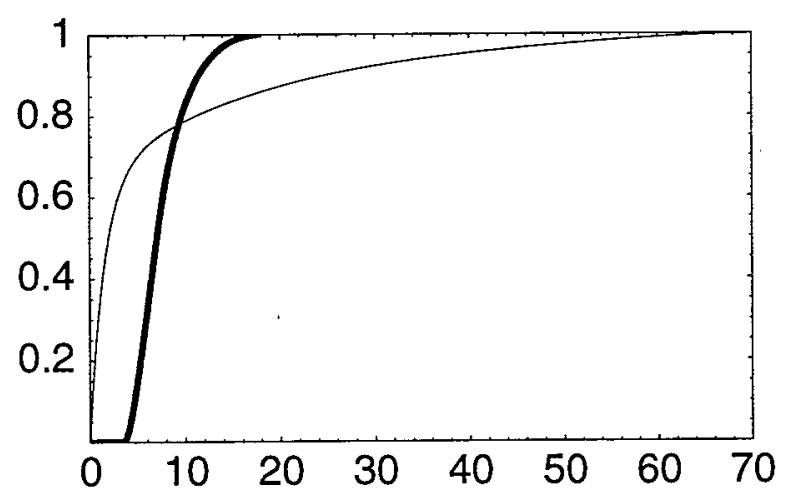

$\{\mathrm{EV}[$ cdfTangbr], $\operatorname{Sqrt}[\operatorname{Var}[$ cdfTangbr] $], \operatorname{Idf}[$ ddfTangbr, $\{.025, .5, .975\}]\}$

$\{7.55321,12.748,\{0.0561443,1.96772,49.7245\}\}$

sdfTbar = standardizecdf [cdfTbar, time $]$;

TBL $[$ out $=$ Prepend $[$ Transpose $[$ \{time, N[Last /e sdfTbar, 8], N[Last /@ cdfTangbr, 8] \}], \{Time, FTbar, FTangbr \}] ]

$\begin{array}{lll}\text { Time } & \text { FTbar } & \text { FTangbr } \\ 0 & 0 & 0.0 \\ 0.1 & 0.000014065155 & 0.044528152 \\ 0.2 & 0.00002813031 & 0.085900584 \\ 0.3 & 0.000042195465 & 0.12439054\end{array}$




\begin{tabular}{|c|c|c|}
\hline 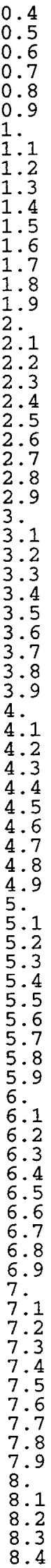 & 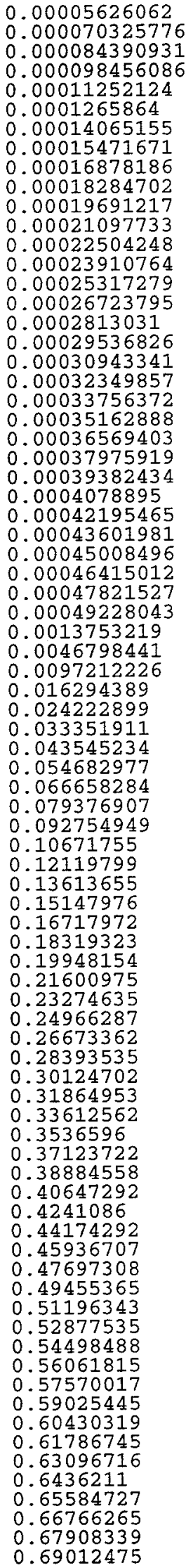 & 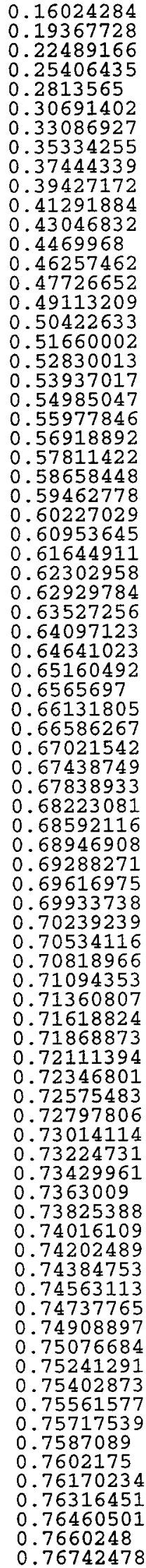 \\
\hline
\end{tabular}




\begin{tabular}{|c|c|c|}
\hline $\begin{array}{l}8.5 \\
8.6 \\
8.7 \\
8.8 \\
8.9 \\
9 . \\
9.1 \\
9.2 \\
9.3 \\
9.4 \\
9.5 \\
9.6 \\
9.7 \\
9.8 \\
9.9 \\
10 . \\
11 \\
12 \\
13 \\
14 \\
15 \\
16 \\
17 \\
18 \\
19 \\
20 \\
21 \\
22 \\
23 \\
24 \\
25 \\
26 \\
27 \\
28 \\
29 \\
30 \\
31 \\
32 \\
33 \\
34 \\
35 \\
36 \\
37 \\
38 \\
39 \\
40 \\
41 \\
42 \\
43 \\
44 \\
45 \\
46 \\
47 \\
48 \\
49 \\
50 \\
55 \\
60 \\
65 \\
70\end{array}$ & $\begin{array}{l}0.70080134 \\
0.71112707 \\
0.72111508 \\
0.73077791 \\
0.74012765 \\
0.74917561 \\
0.75793282 \\
0.7664095 \\
0.77461566 \\
0.78256085 \\
0.79025397 \\
0.79770389 \\
0.80491887 \\
0.81190674 \\
0.81867513 \\
0.82523141 \\
0.88053089 \\
0.9207822 \\
0.9498595 \\
0.97047554 \\
0.98457918 \\
0.99360366 \\
0.99861864 \\
1 . \\
1 . \\
1 . \\
1 . \\
1 . \\
1 . \\
1 . \\
1 . \\
1 . \\
1 . \\
1 . \\
1 . \\
1 . \\
1 . \\
1 . \\
1 . \\
1 . \\
1 . \\
1 . \\
1 . \\
1 . \\
1 . \\
1 . \\
1 . \\
1 . \\
1 . \\
1 . \\
1 . \\
1 . \\
1 . \\
1 . \\
1 . \\
1 . \\
1 . \\
1 . \\
1 . \\
1 . \\
\end{array}$ & 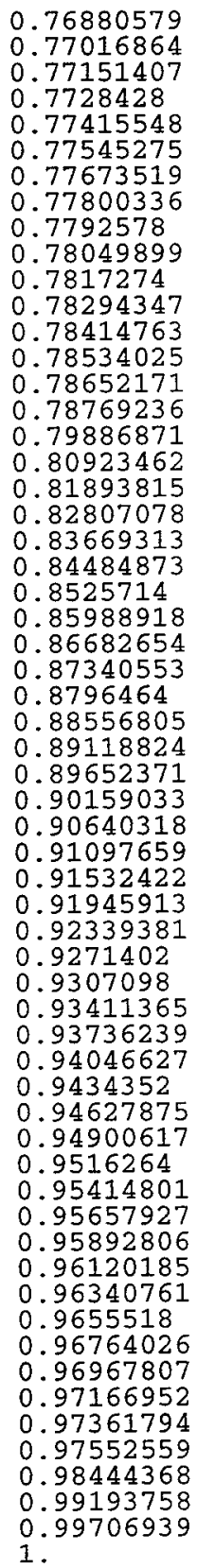 \\
\hline
\end{tabular}

Put [out, PathName [Tbarang] ]; 


\title{
Appendix 3.D Effective Genotoxic Dose
}

\author{
< RiskQ'; \\ HardDrive = "Bogen" ; \\ PathName [filename,, hardDrive_String: HardDrive] := Module [ file = filename , \\ If [mead [file] $=$ ! = String, file = Tostring [file]]; \\ StringJoin [haraDrive, ": Ken:TCE Air Force:Data:", file]];
}

- Input Empirical (Derived) Distributions. Recreate Input Distributions trom Phase Istudy. Exposure in mgkk-d.

\section{- Log-Transform Utility Functions}

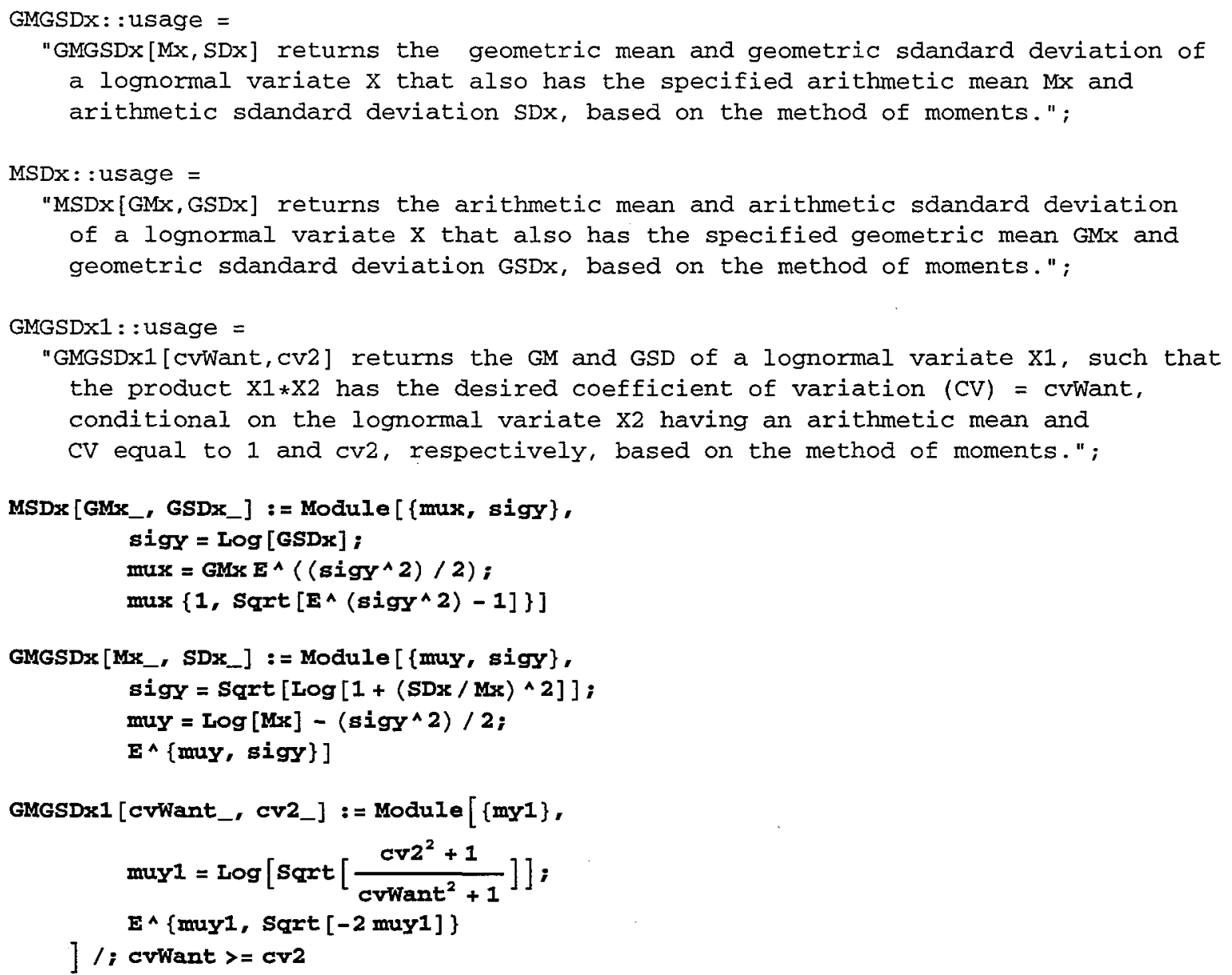




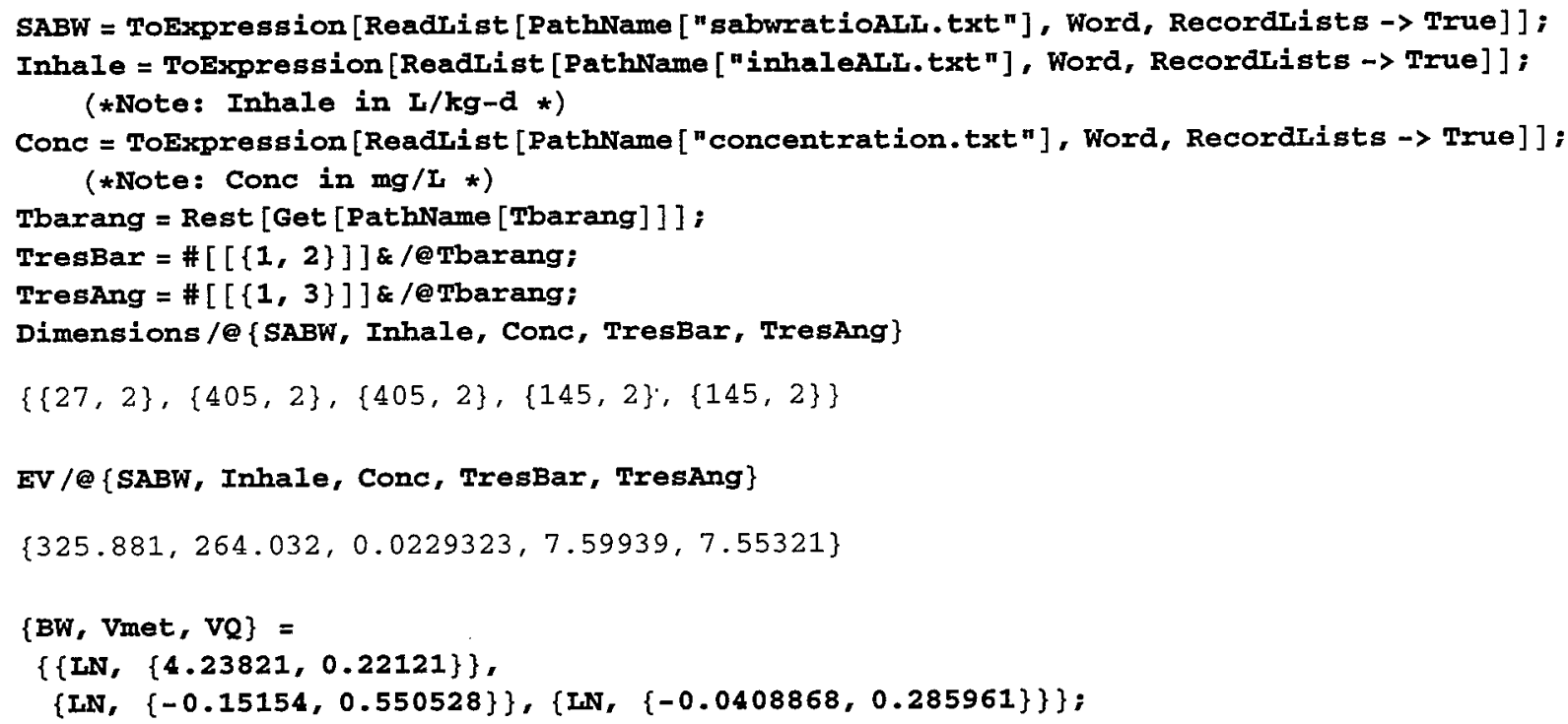

\section{- Constants}

\{TresBarAng, TresAngBar $\}=\operatorname{EV}[\#$, Empirical $\rightarrow$ True $] \& / @\{$ TresBar, TresAng $\}$

$\{7.59939,7.55321\}$

inhalebar $=12.9 * 71^{0.74-1} * \frac{24}{1000} \quad(* \mathrm{~m} 3 / \mathrm{kg}-\mathrm{d} *)$

0.102205

EFcon $=\quad 350 ; \quad(* \mathrm{~d} / \mathrm{y} *)$
ATcon $=\quad 25550 ;(* \mathrm{~d} *)$
ConcAng $=0.0223 ;(* \mathrm{mg} / \mathrm{L} *)$
IngestBar $=0.0242 ;(* \mathrm{~L} / \mathrm{kg}-\mathrm{d} *)$
InhaleBar $=0.102 ;(* \mathrm{~m} 3 / \mathrm{kg}-\mathrm{d} *)$
SABWBar $=325.881 ;(* \mathrm{~cm} / \mathrm{kg} *)$
TresBarAng= $7.59358 ;(* \mathrm{y} *)$
TresAngBar $=7.55321 ;(* \mathrm{y} *)$

end

\section{Fractions metabolized (summary-see "E. Effective Cytotoxic Dose")}

Correlation between Vmet and Fmo $=0.86$

Correlation between VQ and Fmr $=-0.75$

Correlation between Vmet and Fmr $=0.45$

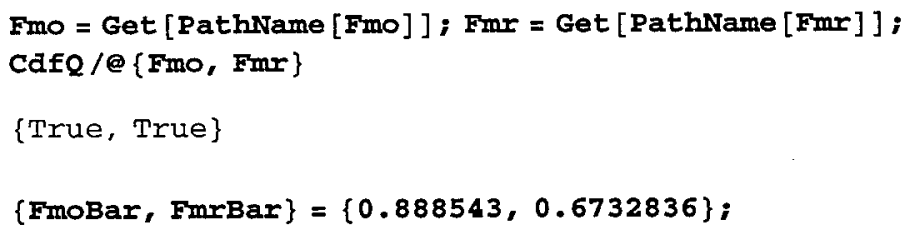


end

Note:

All distributions below are multiplied by Scale $\rightarrow 1000$

\section{- Ingestion}

- EingBar = Uncertainty in Population-Average Level

$\{$ TresBar,Conc $\}=$ uncertain variates

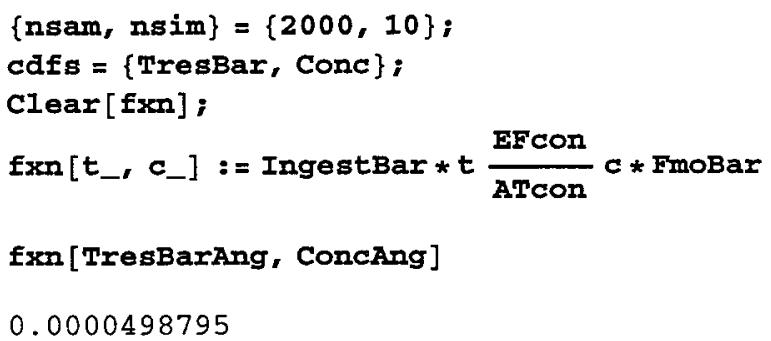


Plotcdf $[\{\mathrm{cvm}, \mathrm{cdf}\}, \mathrm{Ymin} \rightarrow-.01, \mathrm{Xmin} \rightarrow-.0001, \mathrm{Xmax} \rightarrow .125]$;

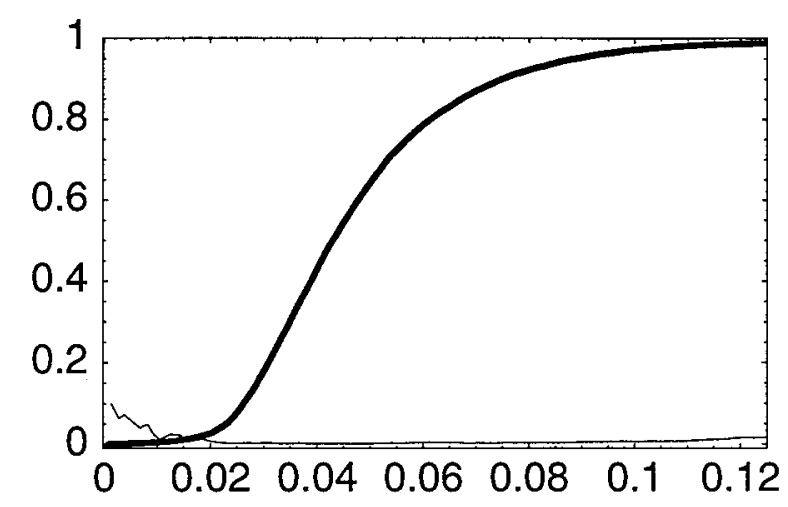

Put [cdf, PathName [EingestBar] ];

end

\section{- <Eing $>$ = Variability in Expected Level}

\{Ingest,TresAng,Fmo $\}=$ heterogeneous variates

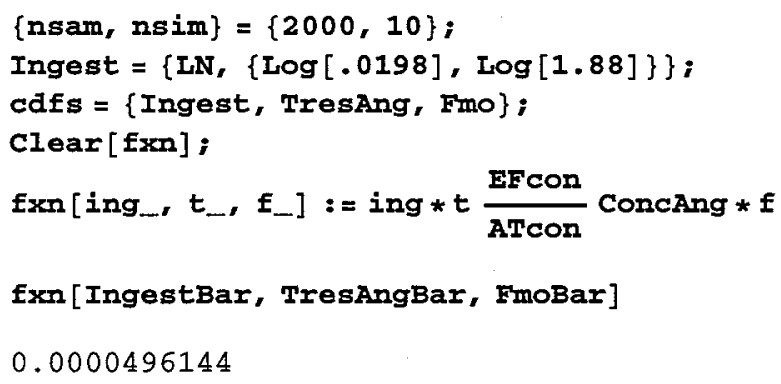




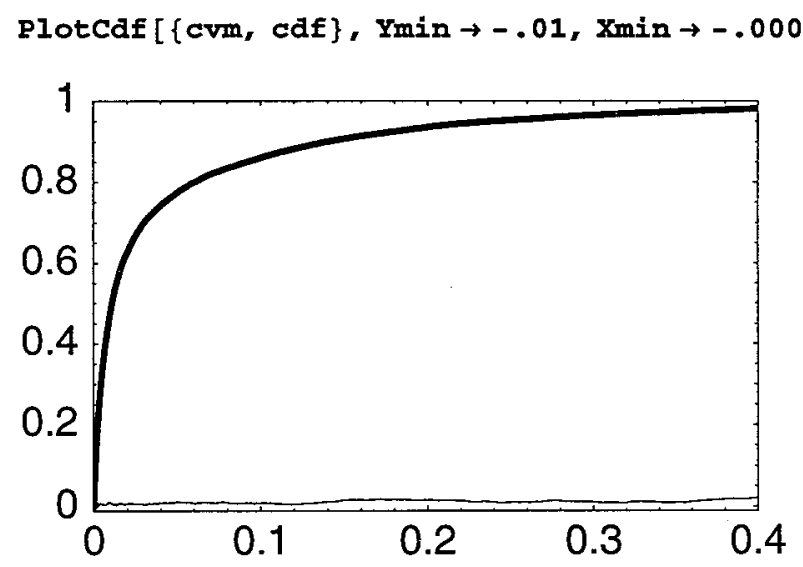

Put [cdf, PathName [EingestAng] ];

end

end

\section{Inhalation Exposure}

\section{- EinhBar = Uncertainty in Population-Average Level}

$\{$ TresBar,Conc $\}=$ uncertain variates

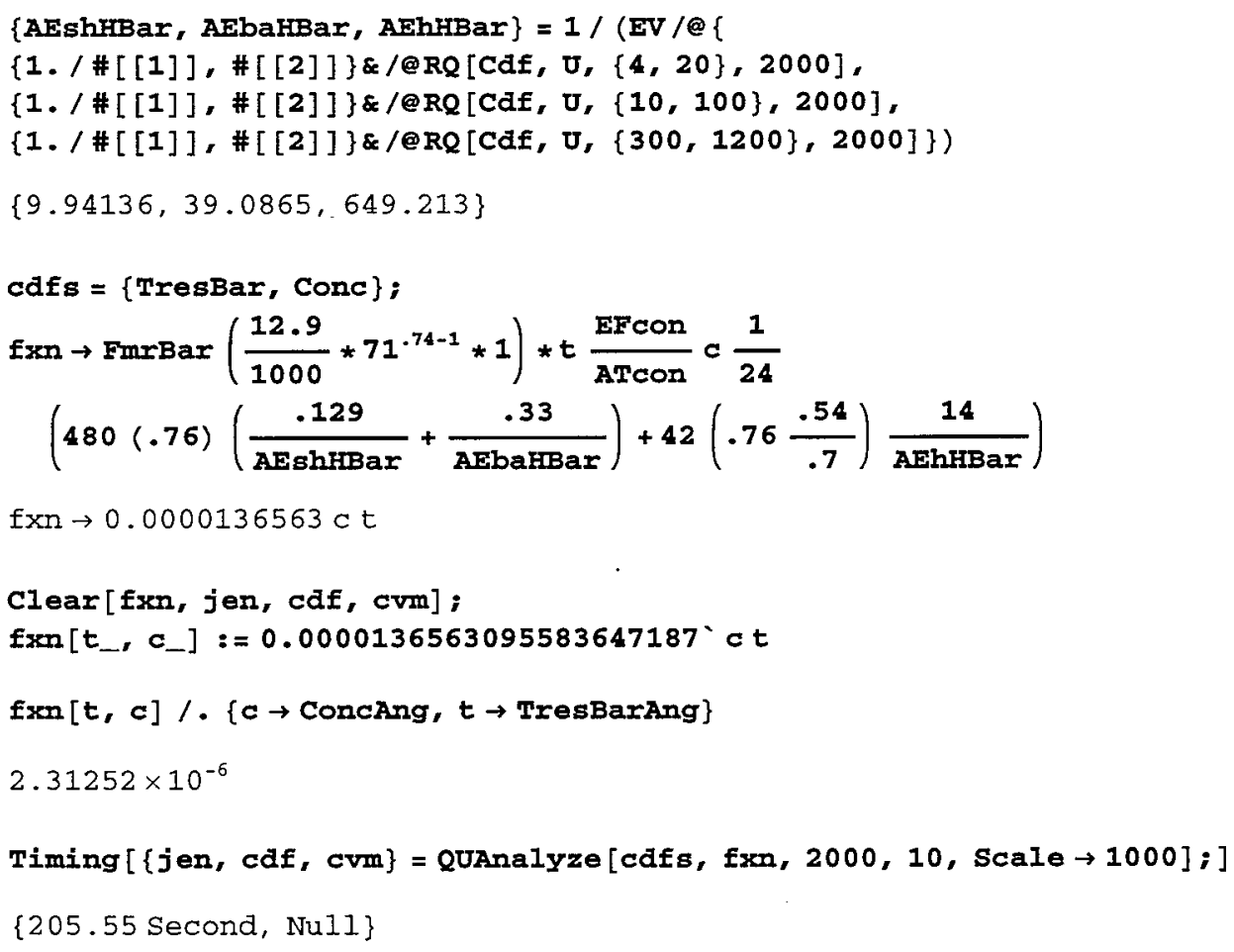




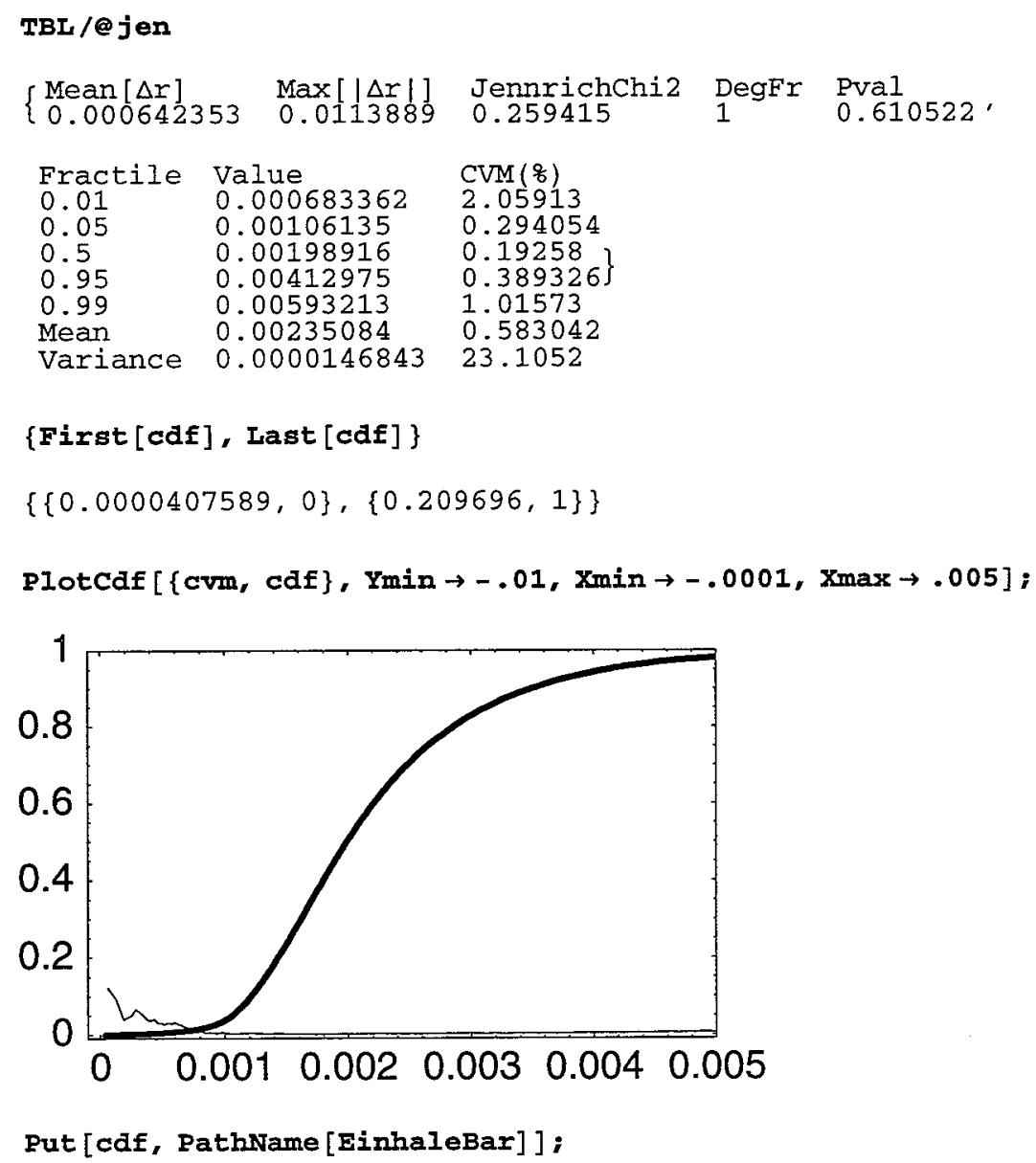

\section{- <Einh $>$ = Variability in Expected Level}

\{Inhale,TresAng,Wshower,Whouse,ETshower,ETbath,EThouse,AEshower,AEbath,AEhouse, $\mathrm{T} 13, \mathrm{~T} 13, \mathrm{VQ}, \mathrm{BW}\}=$ heterogeneous variates

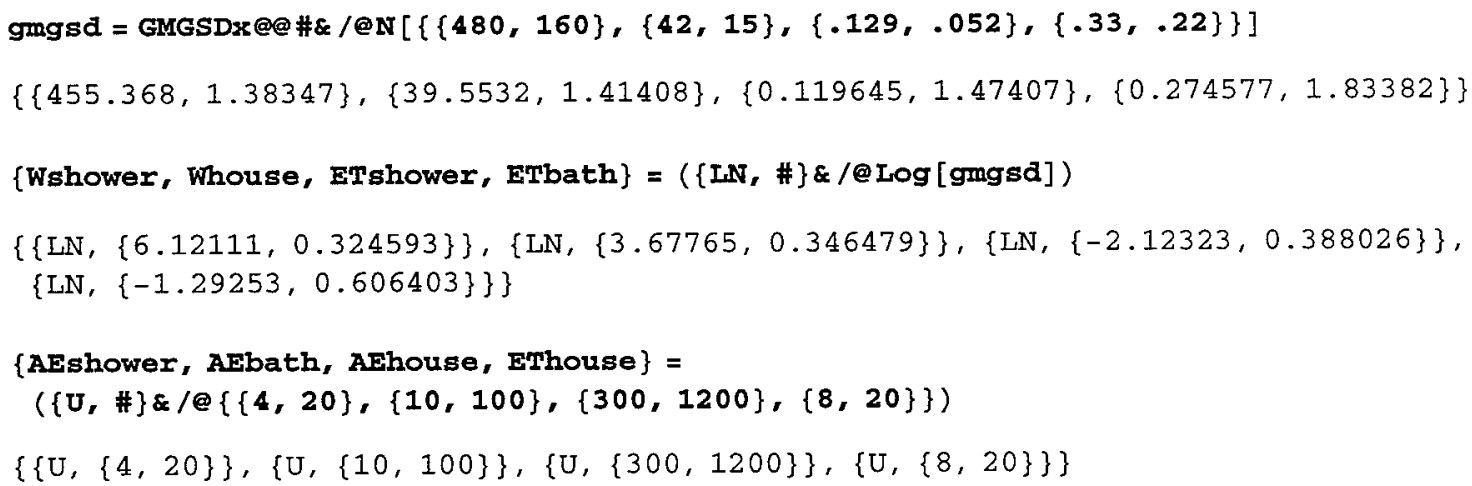


\{InhaleBar, TresAngBar, ConcAng \}

$\{0.102,7.55321,0.0223\}$

$\{\mathrm{VQ}, \mathrm{BW}\}$

$\{\{L N,\{-0.0408868,0.285961\}\},\{L N,\{4.23821,0.22121\}\}\}$

Correlation between VQ and Fmr $=-0.75$ (see "E. Effective Cytotoxic Dose")

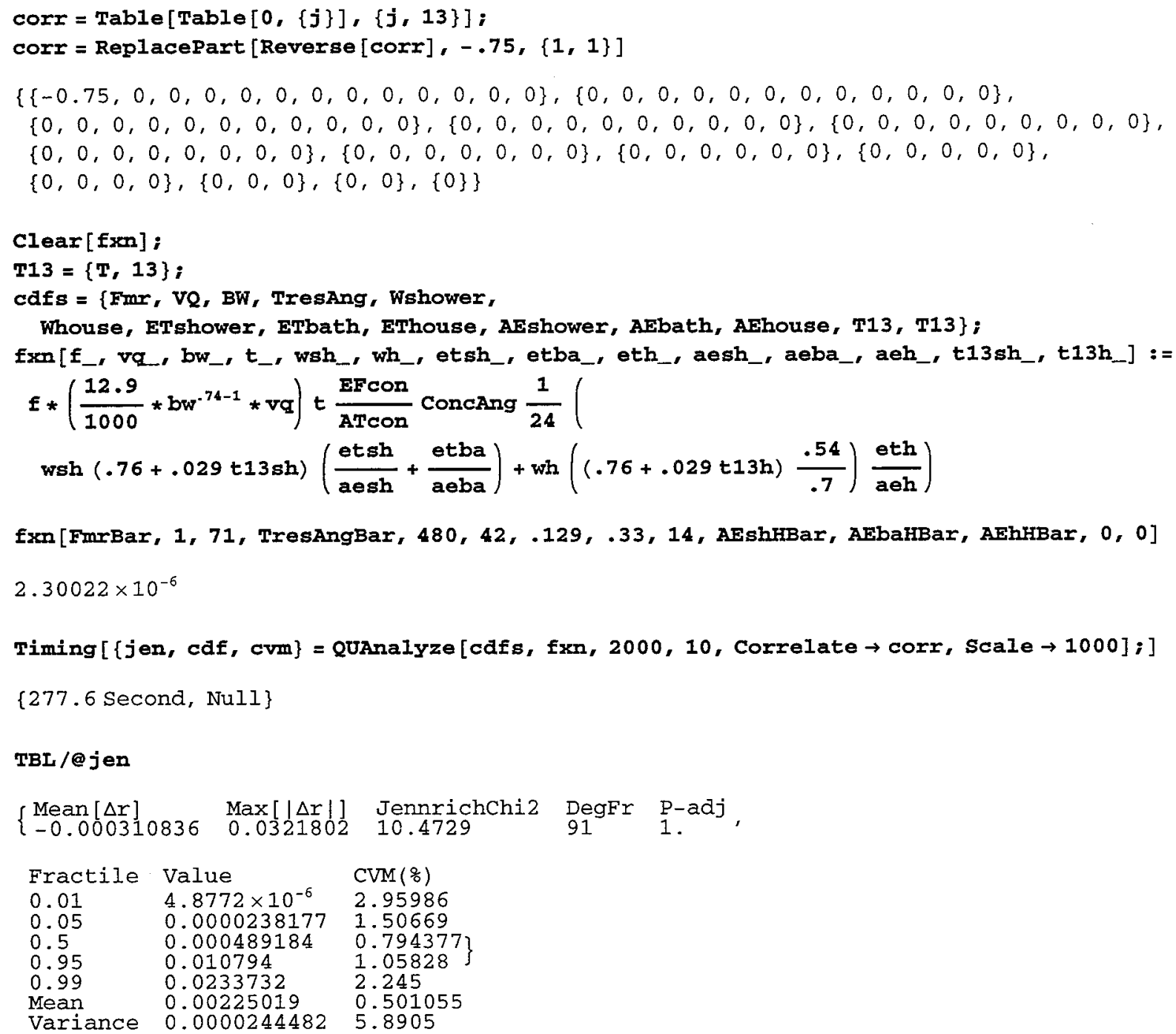




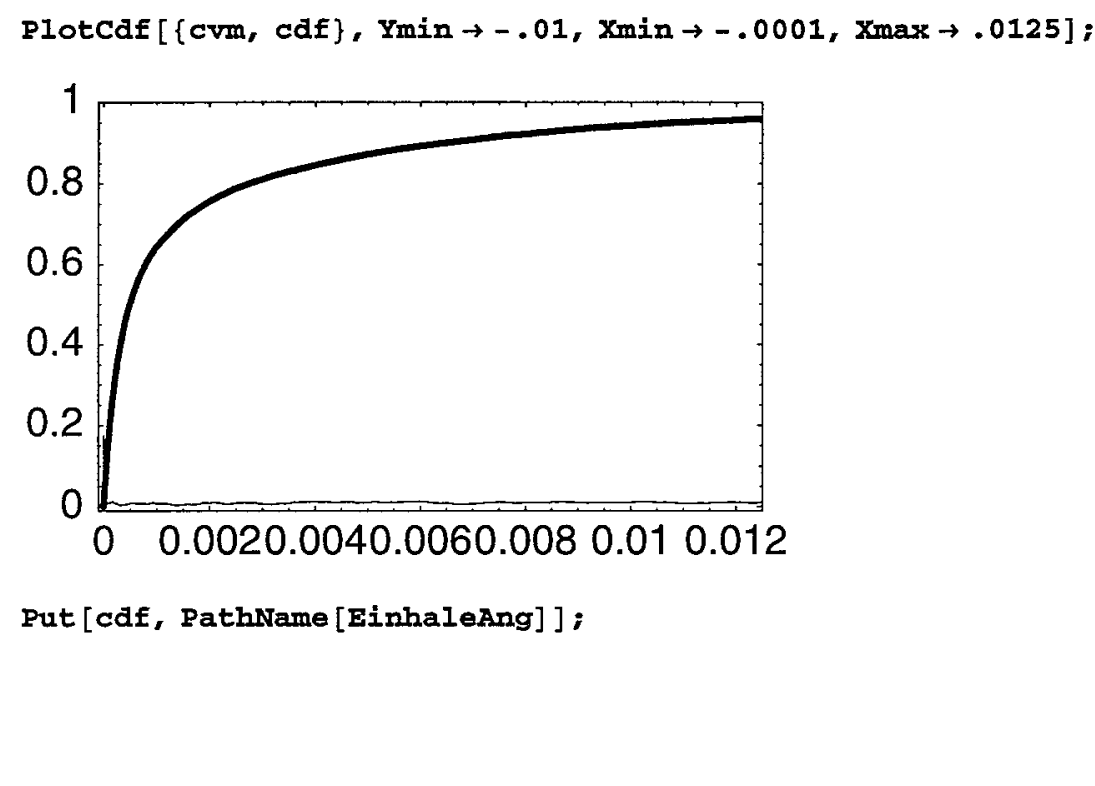

\section{Dermal Exposure}

\section{- EdermalBar = Uncertainty in Population-Average Level}

$\{$ TresBar, Conc $\}=$ uncertain variates

ETshower

$\{L N,\{-2.12323,0.388026\}\}$

cdfs $=\{$ TresBar, Cone $\}$

fxn $\rightarrow$ FmrBar * SABWBar *.65*.263*.129*t $\frac{\text { EFcon }}{\text { ATcon }} c\left(1-\frac{.76}{2}\right) 10^{-3}$

fxn $\rightarrow 0.0000410946 \mathrm{ct}$

Clear $[f x o n]$;

$\operatorname{fxn}\left[t_{-}, c_{-}\right]:=0.0000410945965500063259^{\circ} \mathrm{ct}$

fxen $[t, c] / .\{c \rightarrow$ ConcAng, $t \rightarrow$ TresBarAng $\}$

$6.95883 \times 10^{-6}$

Timing $[\{j e n$, cdf, cvm $\}=$ QUAnalyze [cdfs, fxn, 2000, 10, Scale $\rightarrow 1000]$;

$\{33.3833$ second, NulI\} 


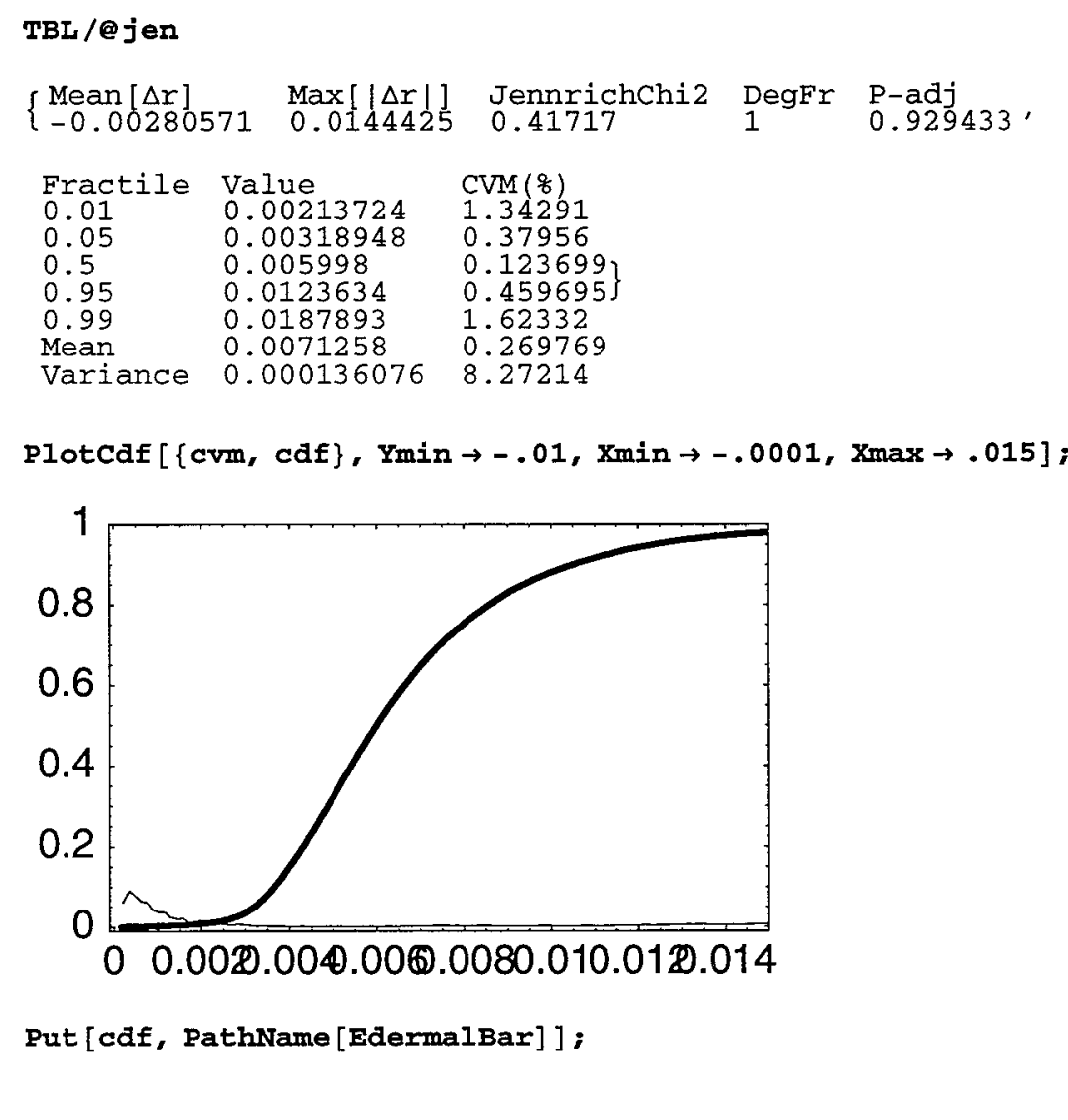

- $<$ Edermal $>=$ Variability in Expected Level

\{SABW,Fs,Kp,ETshower,TresAng,T13,Fmr $\}=$ heterogeneous variates

CafQ/@ SABW, TresAng $\}$

\{True, True\}

\{ConcAng, Fs, KP, ETshower, T13\}

$\{0.0223, \mathrm{Fs}, \mathrm{KP},\{\mathrm{LN},\{-2.12323,0.388026\}\},\{\mathrm{T}, 13\}\}$

$T 13=\{T, 13\} ;$

$\mathbf{F s}=\{\mathbf{U},\{.4, .9\}\}$

$K p=\{N,\{.263, .018\}\} ;$

cdfs $=$ Fmr, SABW, Fs, Kp, ETshower, TresAng, T13\};

Clear [fxen];

$f \times n\left[f_{-}, s a b w_{-}, f s_{-}, k p_{-}\right.$, etsh_, $\left.t_{-}, t 13_{-}\right]:=$

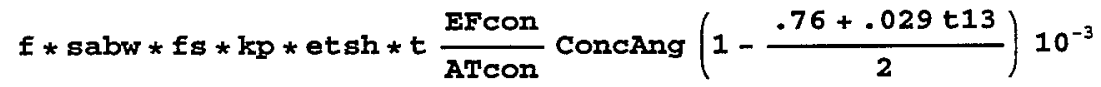

fxn [FmrBar, SABWBar, .65, .263,.129, TresAngBar, 0]

$6.92183 \times 10^{-6}$ 
Timing $[\{j e n$, cdf, cvm $\}=$ QUAnalyze $[$ cdfs, Exn, 2000, 10, Scale $\rightarrow 1000] ;]$

$\{131.933$ second, $\mathrm{Nu} 11\}$

TBI / @ jen

$\left\{\begin{array}{lllll}\text { Mean }[\Delta r] & \text { Max }[|\Delta r|] & \text { JennrichChi2 } & \text { DegFr } & \text { P-adj } \\ -0.000538521 & 0.0183593 & 2.62117 & 21 & 1 .\end{array}\right.$

$\begin{array}{lll}\text { Fractile } & \text { Value } & \text { CVM }(8) \\ 0.01 & 0.0000165555 & 3.56479 \\ 0.05 & 0.0000796803 & 1.07369 \\ 0.5 & 0.00159787 & 0.732505 \\ 0.95 & 0.0331107 & 1.28518 \\ 0.99 & 0.0669827 & 1.42869 \\ \text { Mean } & 0.00684773 & 0.342052 \\ \text { Variance } & 0.000191493 & 1.74394\end{array}$

Plotcdf $[\{\mathrm{cvm}, \mathrm{cdf}\}, \mathrm{Ymin} \rightarrow-.01, \mathrm{Xmin} \rightarrow-.0001, \mathrm{Xmax} \rightarrow .03]$;

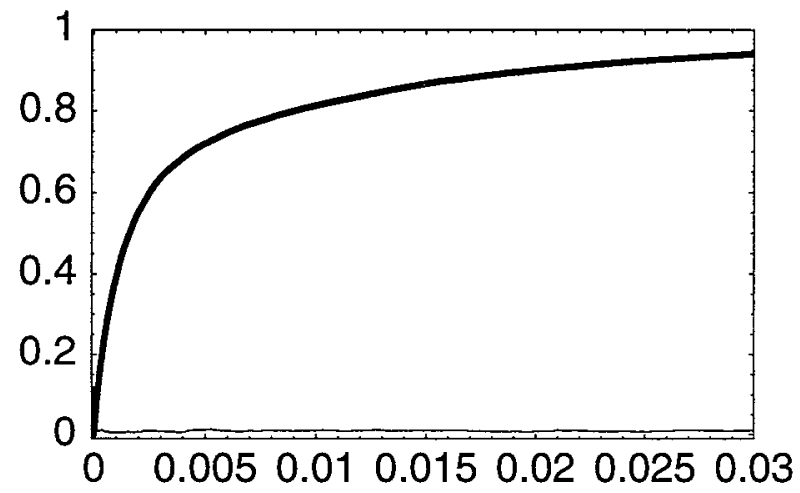

Put [cdf, PathName [EdermalAng] ];

end

end 


\section{Appendix 3.E: Effective Cytotoxic Dose}

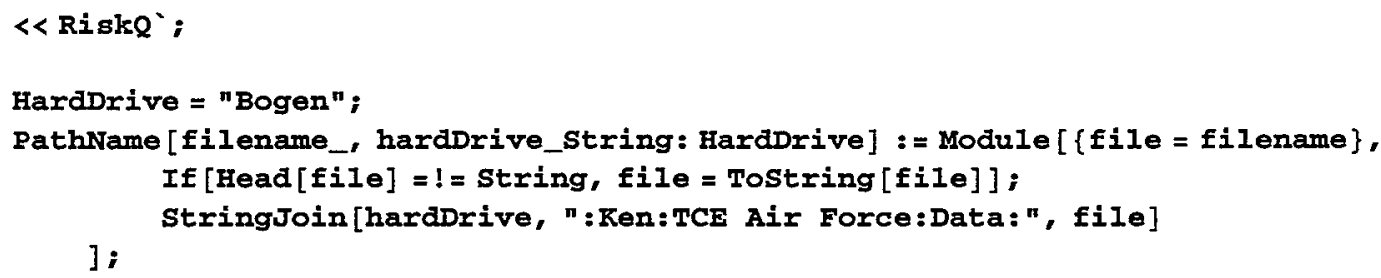

\section{Inputs}

\section{Log-Transform Utility Functions}

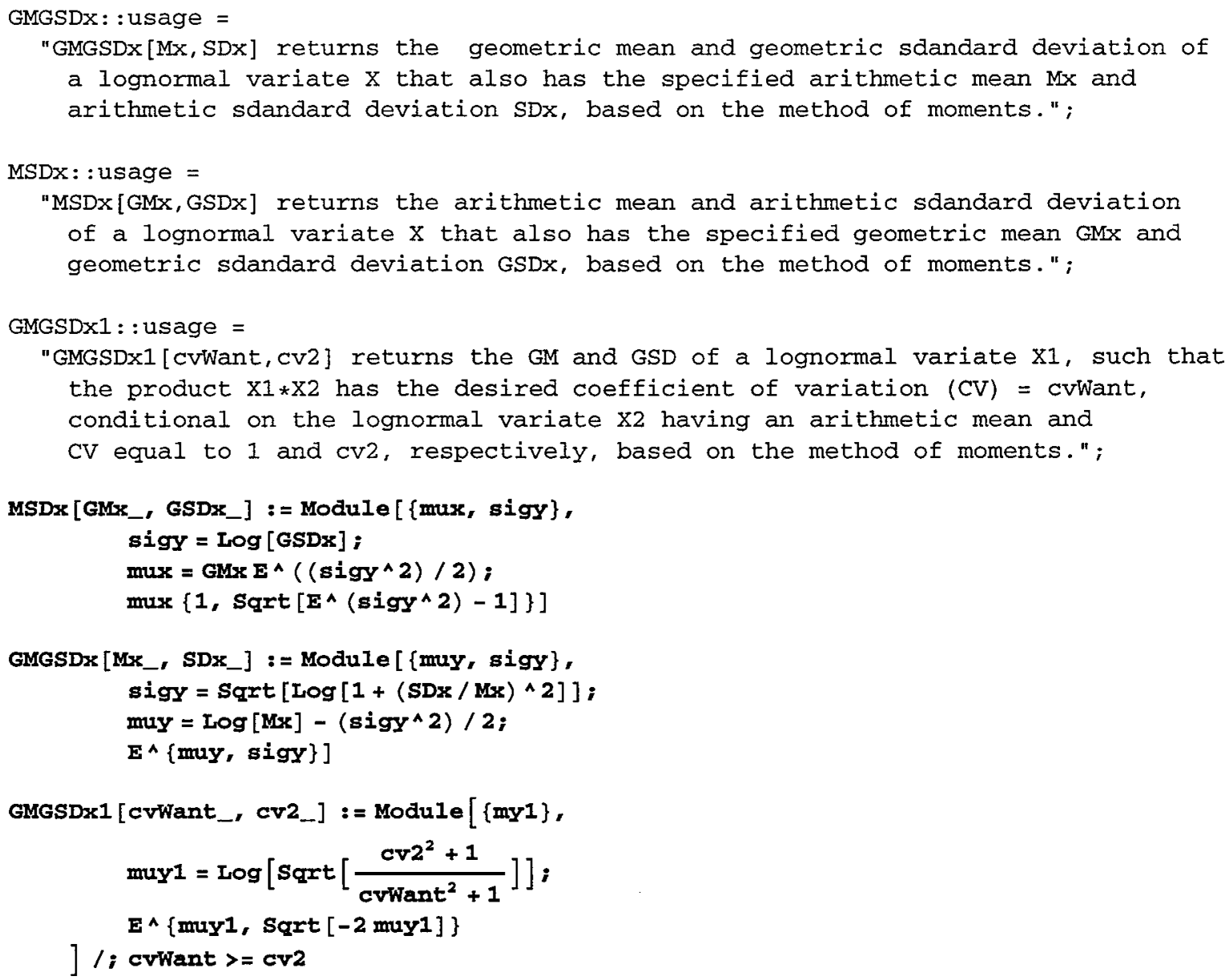




\section{- Input Empirical (Derived) Distributions}

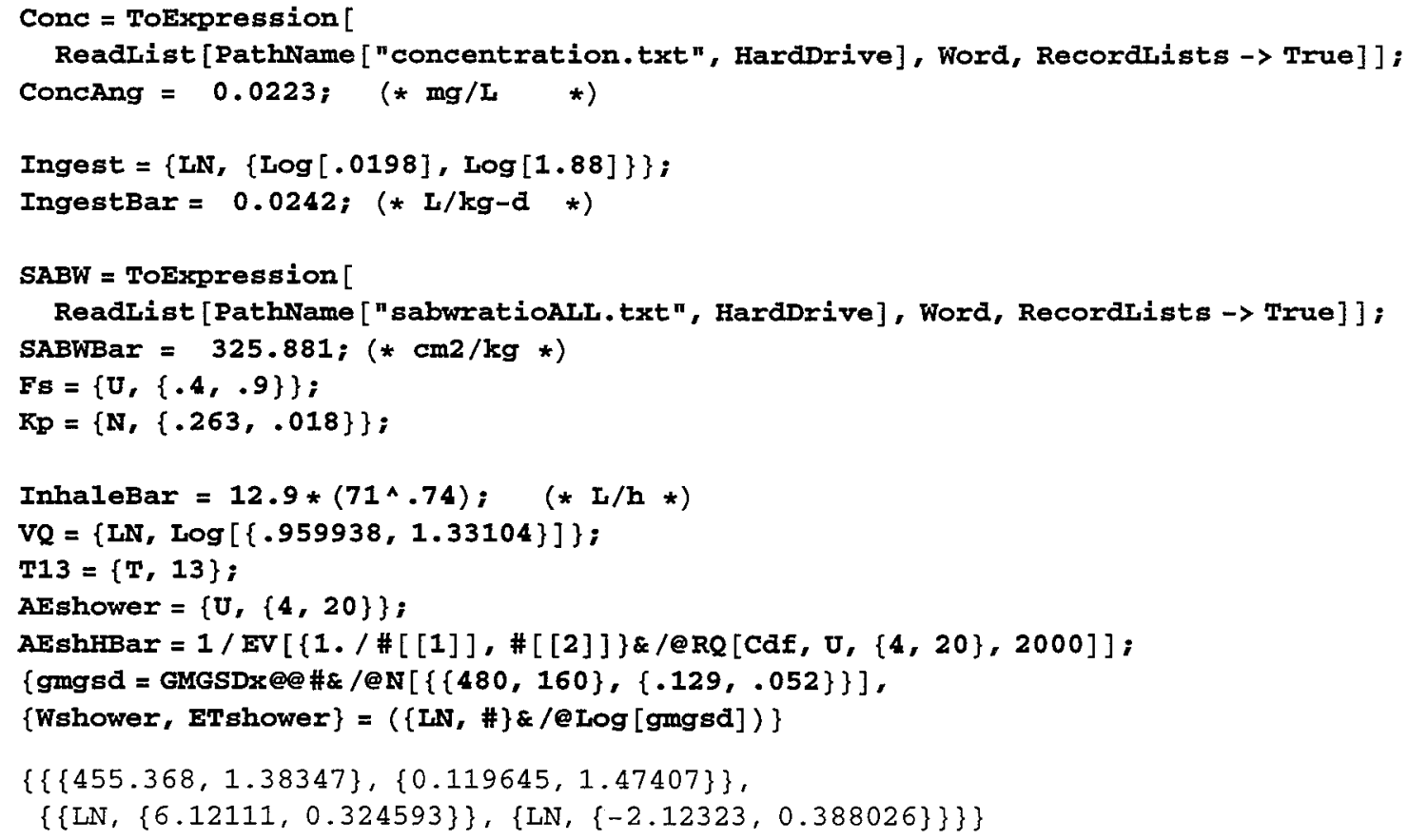

end

\section{Feq (function)}

$\operatorname{Iim}=\operatorname{Limit}\left[\frac{1-E^{-k t}}{t\left(1-E^{-k 24}\right)}, t \rightarrow 0\right]$

$\frac{k}{1-E^{-24 k}}$

$\{1 \mathrm{imk}=1 \mathrm{im} / . \mathrm{k} \rightarrow .000000000001,1 / 1 \mathrm{imk}\}$

$\{0.0416666,24$.

$f x\left[k_{-}, t_{-}\right]:=\frac{1-E^{-k t}}{t\left(1-E^{-k \cdot 24}\right)}$ 
$\operatorname{Plot}\left[\left\{\mathrm{fx}[\mathrm{k}, .01], \operatorname{fx}[\mathrm{k}, .25], \operatorname{fx}[\mathrm{k}, .5], 24^{-1}+.57 \mathrm{k}\right\},\{\mathrm{k}, 0.0001, .1\}\right.$,

Axesorigin $\rightarrow\{0.0001,0.04\}$, PlotRange $\rightarrow\{\{0.0001, .1\},\{0.04, .12\}\}]$;

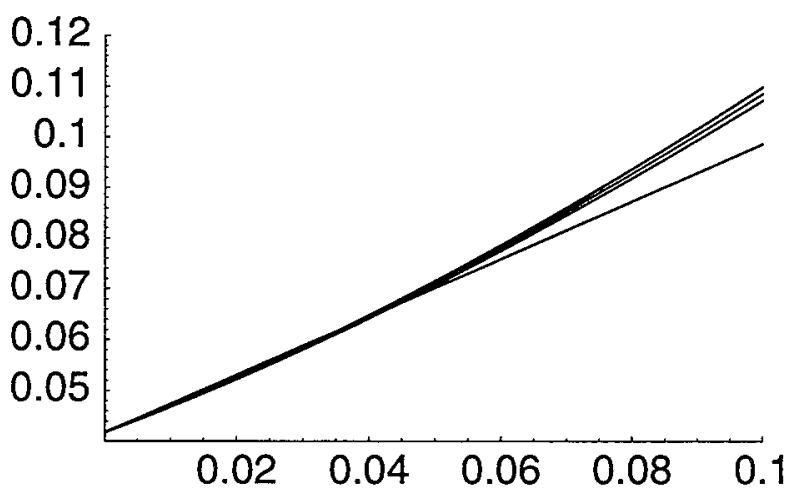

kval $=$ Join $[\{.001\}, \operatorname{Range}[.005, .1, .0025]]$

out $=\{\mathrm{fx}[\mathrm{k}, .01], \mathrm{fx}[\mathrm{k}, .25], \mathrm{fx}[\mathrm{k}, .5]\} / . \mathrm{k} \rightarrow \mathrm{kval}$;

xy = Flatten $\left[\right.$ Transpose $\left[\{\right.$ kval, \# $\} \&$ /@ (out $\left.\left.-24^{\wedge}-1\right), 1\right]$;

fit $=$ Fit $\left[x y,\left\{x, x^{\wedge} 2\right\}, x\right](* k=0-.1 *)$

$0.505316 x+1.66078 x^{2}$

fit $[2]] /$ fit $[[1]]$

$3.28661 \mathrm{x}$

$z z=R Q[Q, N,\{0,1\},\{.95, .99, .995\}] ;$

$\mathrm{cv}=0.60 ;$ (* = assumed $\mathrm{CV}$ for Vke; see below *)

gsd $=E^{\wedge} S g r t\left[\log \left[1+\mathrm{cv}^{\wedge} 2\right]\right] ;$ (* by method of moments *)

$\left\{\mathrm{ke}=0.028 * 71^{\wedge}-.3, \mathrm{gsd}, \mathrm{ke} * \mathrm{gsd}^{\wedge} \mathrm{zz}\right\}$

$\{0.00779436,1.74109,\{0.0194043,0.028315,0.032516\}\}$

data $=(\{0,24 \wedge-1\}+\#) \& / @$ Prepend $[x y,\{0,0\}]$

PlotData [data, FitTo $\rightarrow\left\{\left\{24^{\wedge}-1+\right.\right.$ fit, $\left.\left.24^{\wedge}-1+0.5053 \mathbf{x}\right\}, \mathbf{x}\right\}$,

$\mathrm{Xmin} \rightarrow-.0001, \mathrm{Xmax} \rightarrow .1, Y \min \rightarrow .0399, \mathrm{Ymax} \rightarrow .12$, Style $\rightarrow 00$, Dashed $\rightarrow\{$ False, .025$\}$, Dotsize $\rightarrow$.0125];

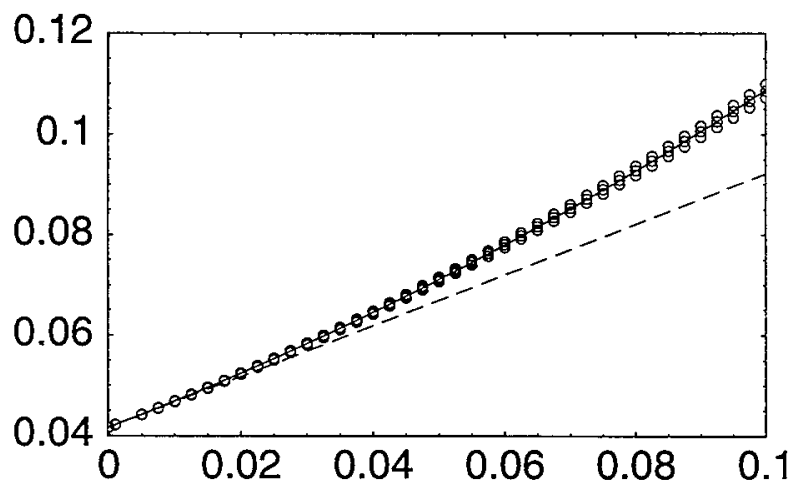




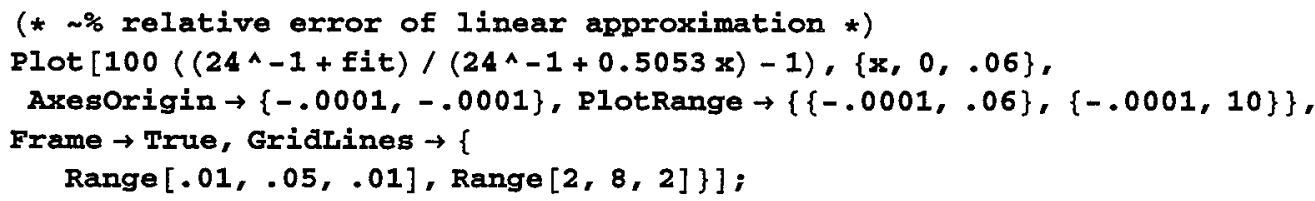

10

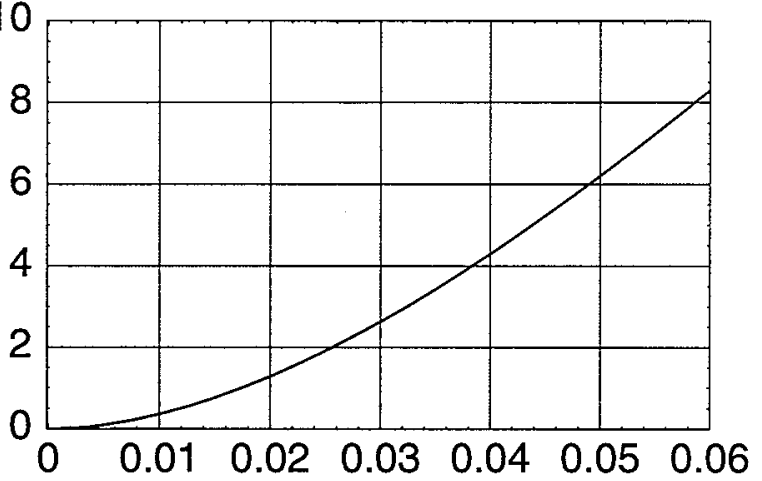

end

\section{Body Weight (adult male + female), $V \max =V e$, and VQ}

$\mathrm{CV}=$ coefficient of variation

$\mathrm{Qa} \quad=$ alveolar ventilation rate $=12.9 * \mathrm{BW}^{\wedge} .74$ (Allen \& Fisher, 1993)

VVmax $=$ Variability (unitless) in Vmax, where $V \max =14.9 * \mathrm{BW}^{\wedge} .74$ (in $\mathrm{mg} / \mathrm{h}$ ) (Allen \& Fisher, 1993)

Vinhale $=$ Variability (unitless) in Inhalation rate, where latter in $\mathrm{L} / \mathrm{h}$

$\mathrm{VKe}=$ Variability (unitless) in $\mathrm{Ke}$, where $\mathrm{Ke}=0.028^{*} \mathrm{BW}^{\wedge}-.3$ (in $1 / \mathrm{h}$ ) (Allen \& Fisher, 1993)

From Finley et al. 1994 (CalEPA 1996, p. 10-7), the BW distribution for adult males \& females is $\sim \mathrm{LN}$ and CV[BW] $=$ - 0.22:

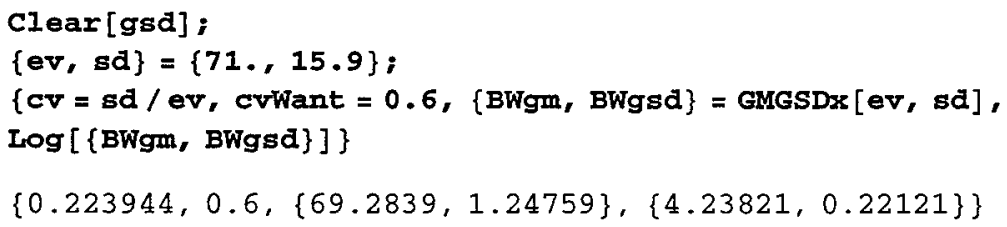

CVwant $=0.60$ for Vmax/BW assumed, based on Lipscombe et al. 1998 (Table 5). From Allen and Fisher (1993), the maximum metabolic rate in $\mathrm{mg} / \mathrm{h}$ is modeled as $\mathrm{Vmax} \sim 14.9 * \mathrm{BW} \mathrm{W}^{\wedge} 0.7$. Now let VVmax be LN-distributed with an arithmetic mean of 1 , where Vmet represents variation in Vmax not attributable to that in BW. Thus, Vmax $14.9^{*} \mathrm{Vmet}^{*} \mathrm{BW}^{\wedge} .7$, whence $\mathrm{Vmax} / \mathrm{BW} \sim 14.9^{*} \mathrm{Vmet}^{*} \mathrm{BW}^{\wedge}-0.3$. It follows from the method of moments that $\mathrm{CV}\left[\mathrm{BW} \mathrm{W}^{\wedge}-.3\right]=$

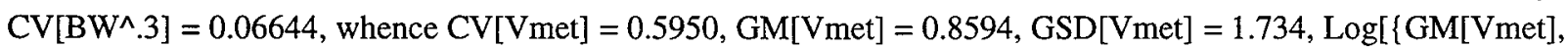
GSD[Vmet] $\}]=\{-0.15154,0.550528\}$. CVwant $=0.60$ for VKe is also assumed, based on Fisher et al. 1998 (Table 8); thus $\mathrm{VKe}=\mathrm{VVmax}=\mathrm{Vmet}$.

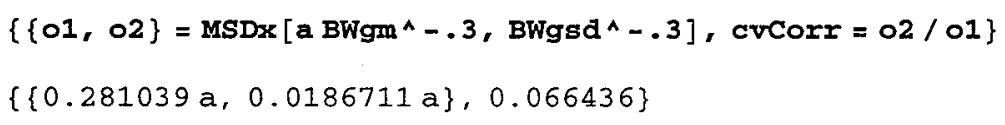




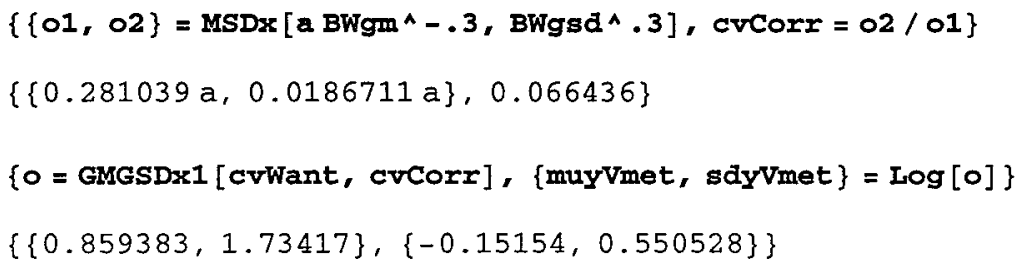

From CalEPA/OHEA (1996, Stochastic Analysis, p. 3-31 - 3-32; cit. above), cvA = CV[24*Q/BW] = CV[Qtot/BW] = 0.3 , where $Q$ denotes total ventilation rate in $\mathrm{L} / \mathrm{h}$. From Allen and Fisher (1993), alveolar ventilation rate in $\mathrm{L} / \mathrm{h}$ is modeled as $\mathrm{Q} \sim 12.9^{*} \mathrm{BW} \mathrm{W}^{\wedge} 0.7$, and Qtot $\sim \mathrm{kQ}$ for some constant $\mathrm{k}$. Now let VQ be LN-distributed with an arithmetic mean of 1 , where VQ represents variation in $\mathrm{Q}$ not attributable to that in $\mathrm{BW}$. Thus, $\mathrm{Q} \sim 12.9 * \mathrm{VQa} \mathrm{BW}^{\wedge} .7$, whence $\mathrm{Q} / \mathrm{BW} \sim$ $12.9 * \mathrm{VQ}^{*} \mathrm{BW}^{\wedge}-0.3$. It follows from the method of moments that $\mathrm{CV}\left[\mathrm{BW}^{\wedge}-.3\right]=\mathrm{CV}\left[\mathrm{BW} \mathrm{N}^{\wedge} .3\right]=0.06644$, whence $\mathrm{CV}[\mathrm{VQ}]=$ $0.2919, \mathrm{GM}[\mathrm{VQ}]=0.9599, \mathrm{GSD}[\mathrm{VQ}]=1.331, \log [\{\mathrm{GM}[\mathrm{VQ}], \mathrm{GSD}[\mathrm{VQ}]\}]=\{-0.0408868,0.285961\}$.

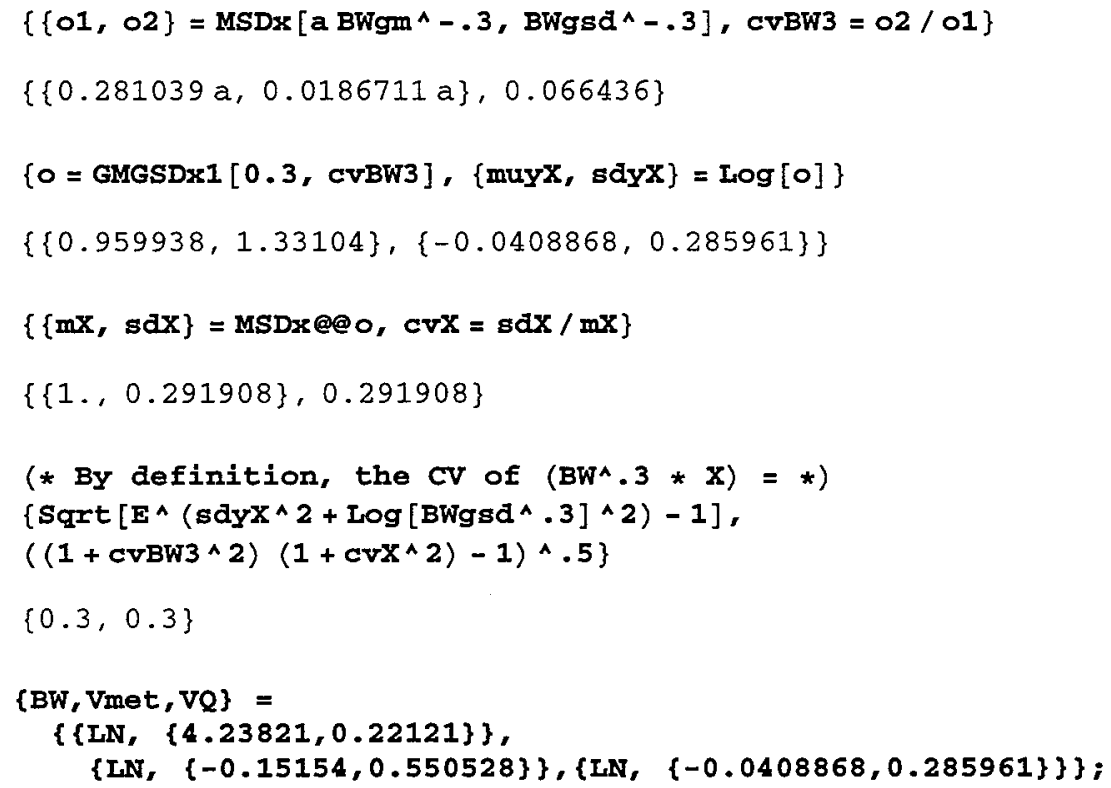




\section{- Fractions metabolized}

\section{- Oral (fmo)}

Heterogeneous variates $=\{\mathrm{Pb}, \mathrm{Vmet}, \mathrm{VQ}\}$

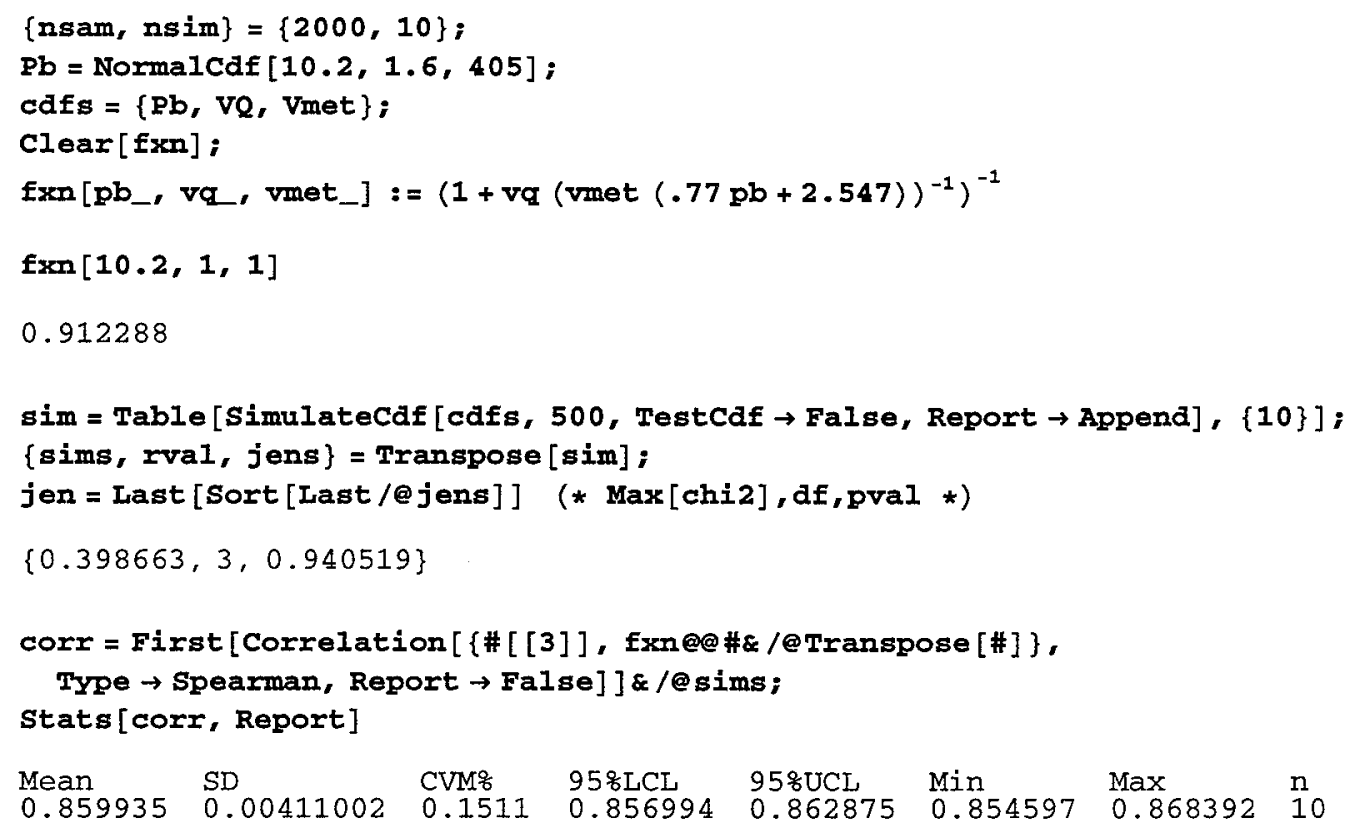

Correlation between Vmet and Fmo $=0.86$

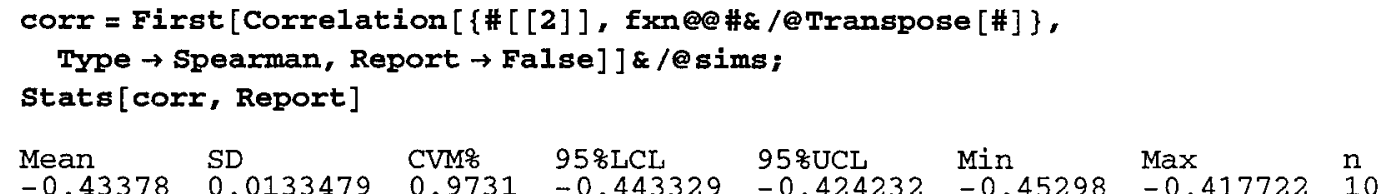

Correlation between VQ and Fmo $=-0.43$

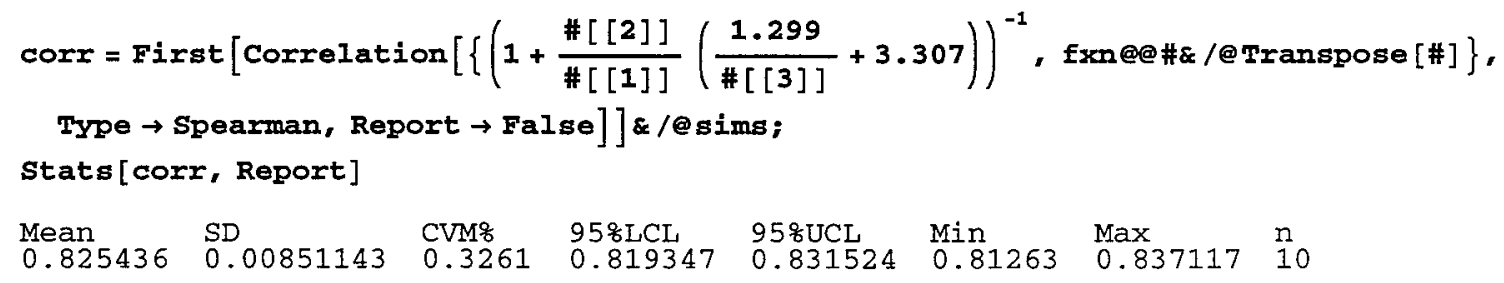

Correlation between Fmr and Fmo $=0.83$

Timing $[\{j e n$, cdf, cvm $\}=$ QUAnalyze $[\mathrm{cdfs}$, fxn, nsam, nsim, scale $\rightarrow 1] ;]$

$\{1094.55$ second, Nul1\} 

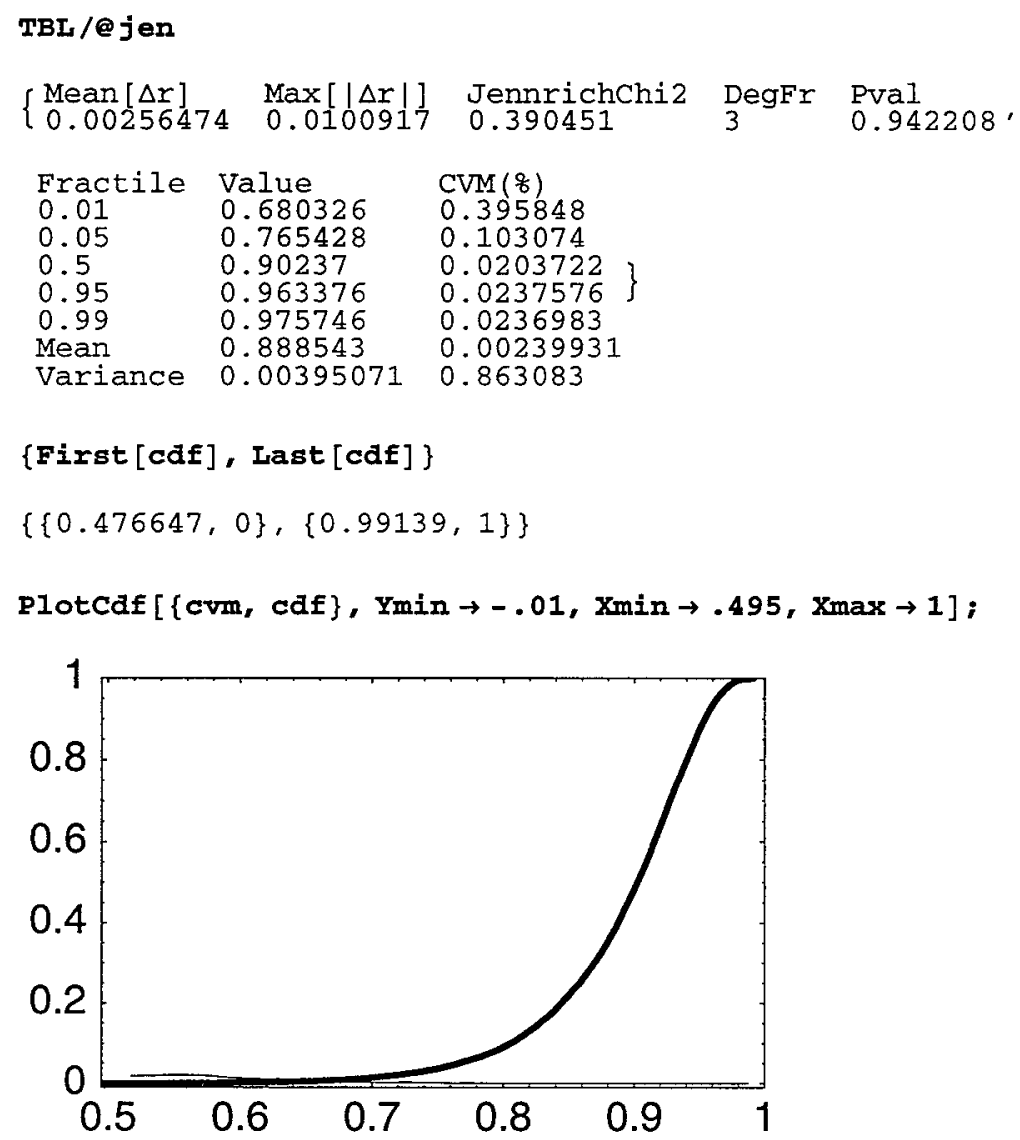

Fmo = Standardizecdf $[$ cdf, 405];

end

\section{- Inhalation and dermal (fmr)}

Heterogeneous variates $=\{\mathrm{Pb}, \mathrm{Vmet}, \mathrm{VQ}\}$

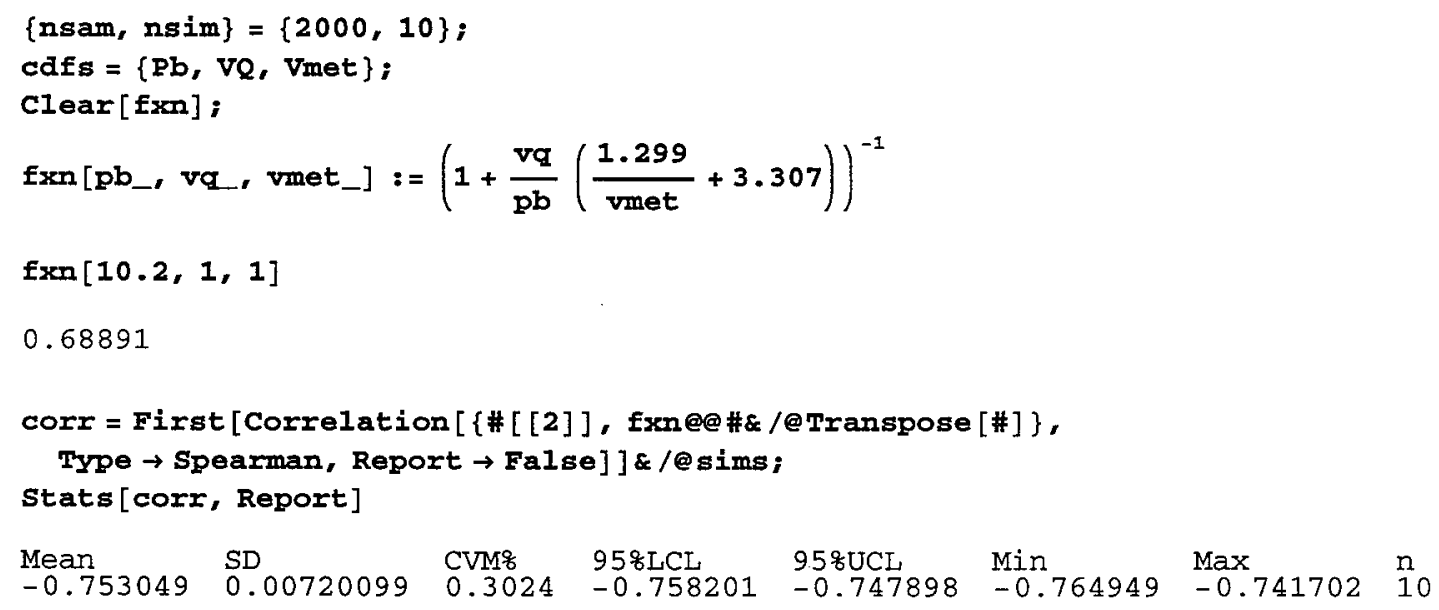


Correlation between VQ and $\mathrm{Fmr}=-\mathbf{0 . 7 5}$

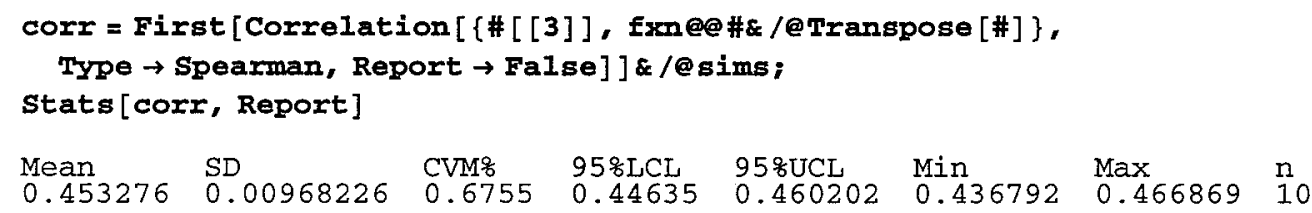

Correlation between Vmet and Fmr $=0.45$

Timing $[\{j e n$, cdf, cvm $\}=$ QUAnalyze [cdfs, fxm, nsam, nsim, Scale $\rightarrow 1] ;]$

$\{1096.72$ Second, Nul1 $\}$

TBL/@jen

\begin{tabular}{|c|c|c|c|c|c|c|c|}
\hline$\left\{\begin{array}{l}\text { Mean }[\Delta r] \\
0.001593\end{array}\right.$ & $\begin{array}{l}\operatorname{Max}[|\Delta r|] \\
0.0140407\end{array}$ & $\begin{array}{l}\text { JennrichChi2 } \\
0.499243\end{array}$ & $\frac{D e g F r}{3}$ & $\begin{array}{l}\text { Pval } \\
0.919058^{\prime}\end{array}$ & $\begin{array}{l}\text { Fractile } \\
0.01 \\
0.05 \\
0.5 \\
0.95 \\
0.99 \\
\text { Mean } \\
\text { Variance }\end{array}$ & $\begin{array}{l}\text { Value } \\
0.454963 \\
0.527838 \\
0.680315 \\
0.794697 \\
0.830531 \\
0.673284 \\
0.00656651\end{array}$ & $\begin{array}{l}\text { CVM }(8) \\
0.559908 \\
0.151762 \\
0.0680913 \\
0.0632056 \\
0.090292 \\
0.0011681 \\
0.240044\end{array}$ \\
\hline
\end{tabular}

\{First [cdf], Last [cdf]

$\{\{0.264383,0\},\{0.890069,1\}\}$

Plotcdf $[\{c v m, c d f\}, Y \min \rightarrow-.01, \mathrm{Xmin} \rightarrow-.0001, \mathrm{Xmax} \rightarrow 1]$;

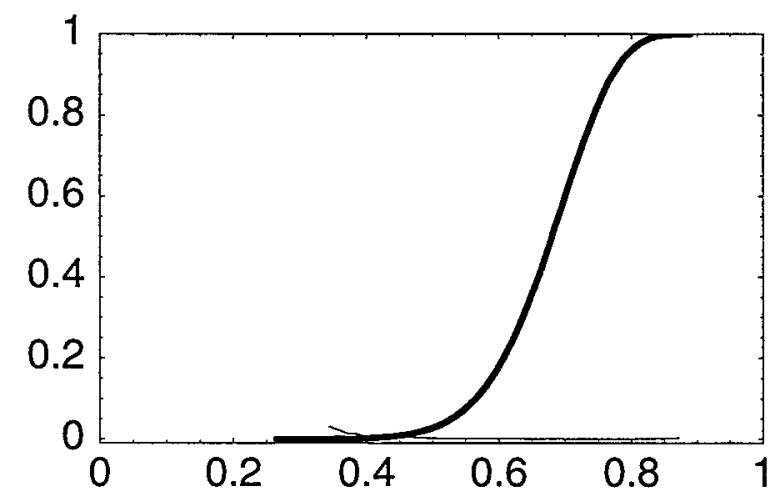

Fmr = Standardizecdf $[\mathrm{cdf}, 405]$

end

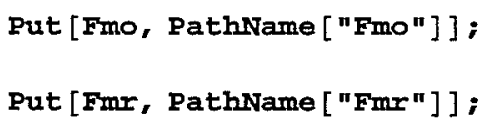

Correlation between Vmet and Fmo $=0.86$

Correlation between VQ and $\mathrm{Fmr}=-0.75$

Correlation between Vmet and Fmr $=0.45$ 


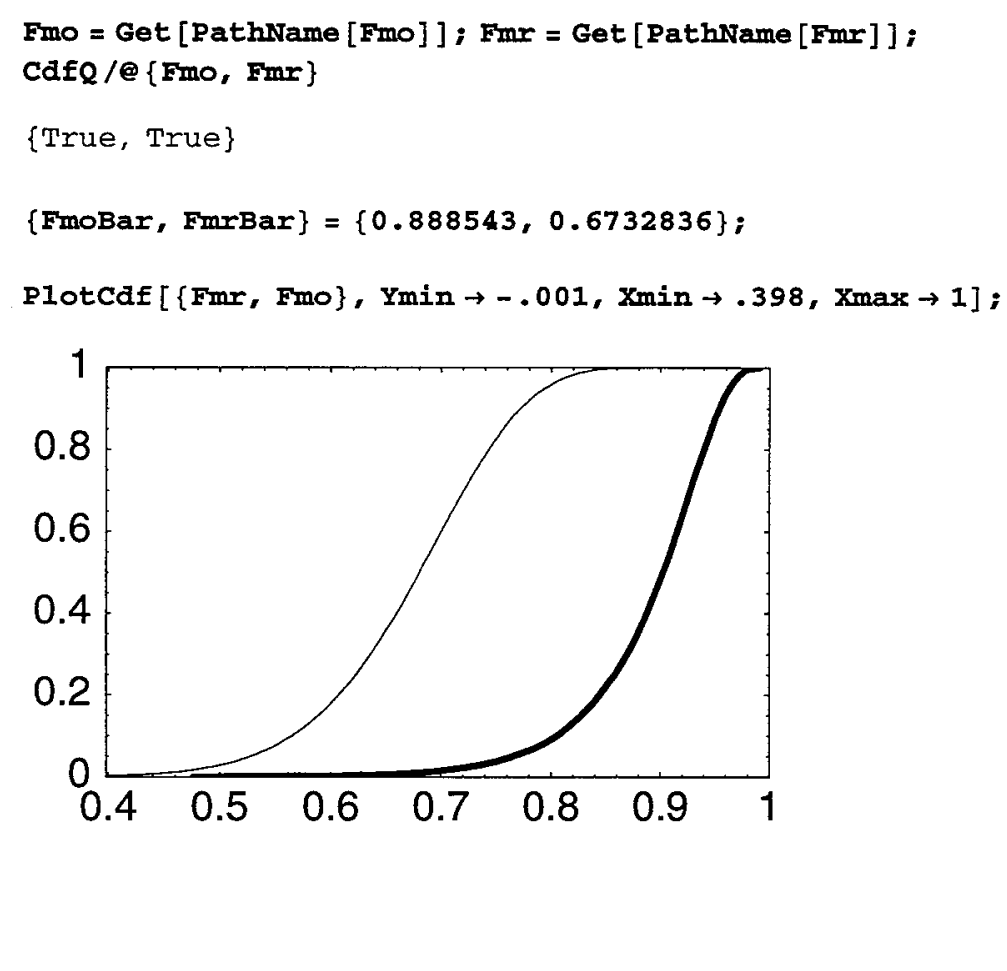

Note: All distributions below are unscaled

\section{Ingestion Effective Dose (mg TCA/L plasma)}

- $<$ ECingest $>$ = Variability in Expected Level

\{VolDist,BW,VKe,Fmo,Ingest $\}=$ heterogeneous variates

Correlation between VolDist and BW is assumed to be -.5

Correlation between Vmet $=\mathrm{Ve}$ and Fmo is assumed to be 0

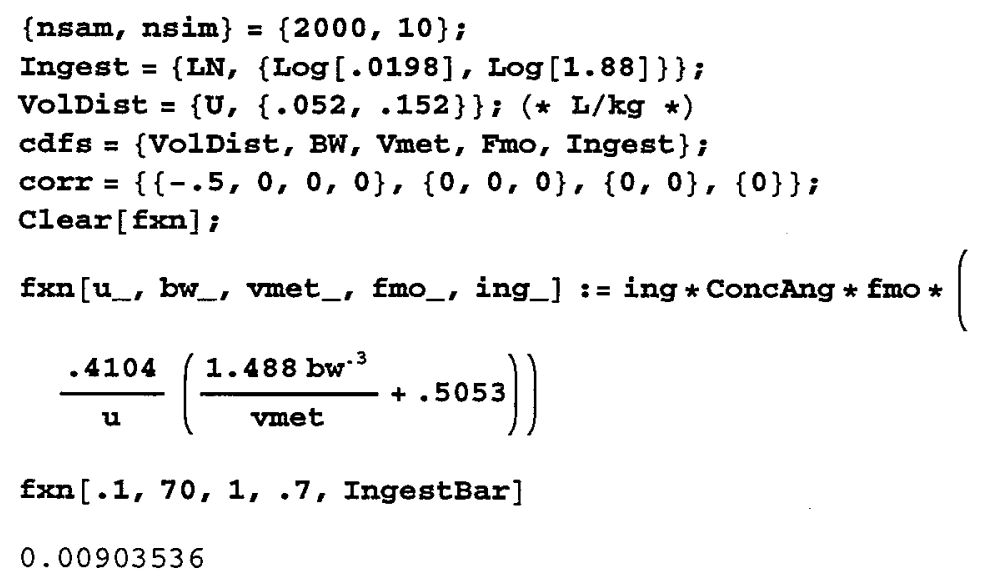


Timing $[\{j e n, c d f, c v m\}=$ QUAnalyze $[c d f s$, fxn, nsam, nsim, Scale $\rightarrow 1$, Correlate $\rightarrow$ corr $] ;]$ $\{85.7333$ second, Nul1\}

TBL / jen

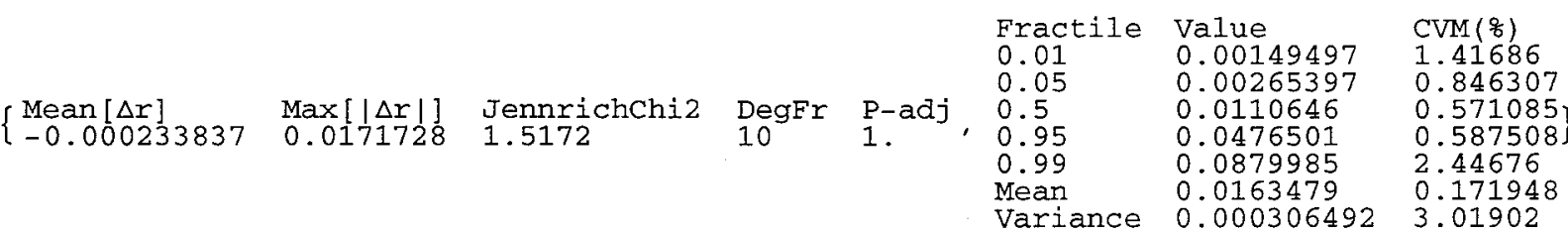

PlotCdf $[\{c v m, c d f\}, Y \min \rightarrow-.01, \mathrm{Xmin} \rightarrow-.0001, \mathrm{Xmax} \rightarrow .07]$;

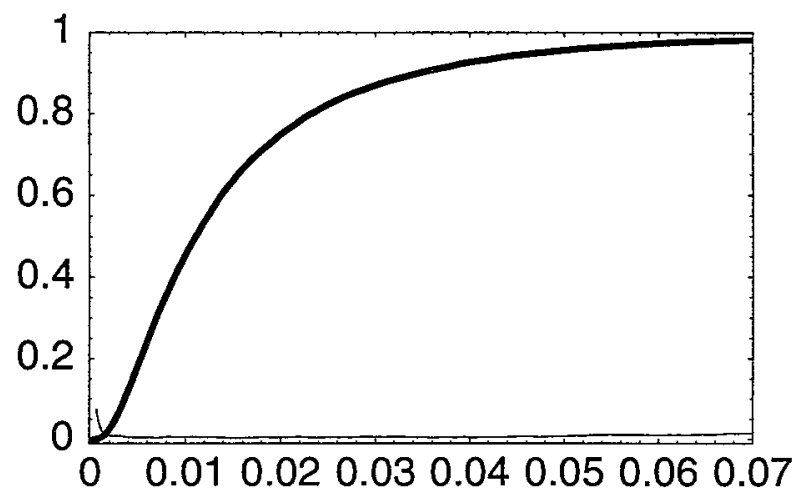

sdf = Standardizecdf [cdf, 404]; Ev [sdf, Empirical -> True $]$

0.0161848

Put [sdf, PathName [ECingestAng] ];

ECingestAng $=$ Get $[$ PathName [ECingestAng $]]$;

ECingestangBar $=0.0161848$;

end

\section{- ECingestBar = Uncertainty in Population-Average Level}

$\{$ Conc $\}=$ uncertain variate

$$
\begin{aligned}
& \text { cdf }=\left\{\frac{\text { ECingestAngBar }}{\text { ConcAng }} \#[[1]], \#[[2]]\right\} \& / @ \text { Conc; } \\
& \text { Put }[\text { cdf, PathName }[\text { ECingestBar }]] ; \\
& \text { ECingestBar }=\text { Get }[\text { PathName }[\text { ECingestBar }]] ;
\end{aligned}
$$

end

end 


\section{- Inhalation Effective Dose (mg TCA/L plasma)}

\section{- <ECinhale $>$ = Variability in Expected Level}

\{VolDist,BW,VKe,Fmr,Inhale,Wshower,ETshower,AEshower,T13 $\}=$ heterogeneous variates Correlation between Vmet $=\mathrm{Ve}$ and $\mathrm{Fmr}$ is assumed to be 0 Correlation between VolDist and BW is assumed to be -.5 Correlation between VQ and Fmr $=-0.75$

\{InhaleBar, ConcAng\}

$\{302.358,0.0223\}$

\{nsam, nsim $\}=\{2000,10\}$;

Clear [ $\mathrm{fxn}]$;

cdfs $=\{$ VolDist, BW, Vmet, VQ, Fmr, Wshower, ETshower, AEshower, T13\}; $\operatorname{corr}=\{\{-.5,0,0,0,0,0,0,0\},\{0,0,0,0,0,0,0\}$,

$\{0,0,0,0,0,0\},\{-.75,0,0,0,0\},\{0,0,0,0\},\{0,0,0\},\{0,0\},\{0\}\} ;$

$\operatorname{fxn}\left[u_{-}, b w_{-}, v m e t_{-}, v q, f m r_{-}, w s h_{-}, e t s h_{-}, a e s h_{-}, t 13_{-}\right]:=\left(\frac{.4104}{u}\left(\frac{19.195}{v m e t}+\frac{6.5145}{b w^{.3}}\right)\right.$

vq * ConcAng $\left(\frac{\text { wsh }(.76+.029 t 13)}{1000 \text { aesh }}\right) \operatorname{fmr} *$ etsh

$\operatorname{fxn}[.1,70,1,1, .7,480, .129$, AEshHBar, 0]

0.00637328

Timing $[\{$ jen, cdf, cvm $\}=$ QUAnalyze [cdfs, fxn, nsam, nsim, scale $\rightarrow 1$, Correlate $\rightarrow$ corr $] ;]$ $\{179.167$ Second, Nul1\}

TBL/@jen

$\left\{\begin{array}{lllll}\text { Mean }[\Delta r] & \text { Max }[|\Delta r|] & \text { JennrichChi2 } & \text { DegFr } & \text { P-adj } \\ -0.000248172 & 0.0207595 & 4.33342 & 36 & 1 .\end{array}\right.$

$\begin{array}{lll}\text { Fractile } & \text { Value } & \text { CVM }(8) \\ 0.01 & 0.000715826 & 1.62597 \\ 0.05 & 0.00126666 & 0.60722 \\ 0.5 & 0.00538319 & 0.46861 \\ 0.95 & 0.0259794 & 1.09888 \\ 0.99 & 0.0489231 & 1.70975 \\ \text { Mean } & 0.00844442 & 0.179915 \\ \text { Variance } & 0.0000944227 & 3.53902\end{array}$


Plotcdf $[\{\mathrm{cvm}, \mathrm{cdf}\}, \mathrm{Ymin} \rightarrow-.01, \mathrm{Xmin} \rightarrow-.0001, \mathrm{Xmax} \rightarrow .05]$;

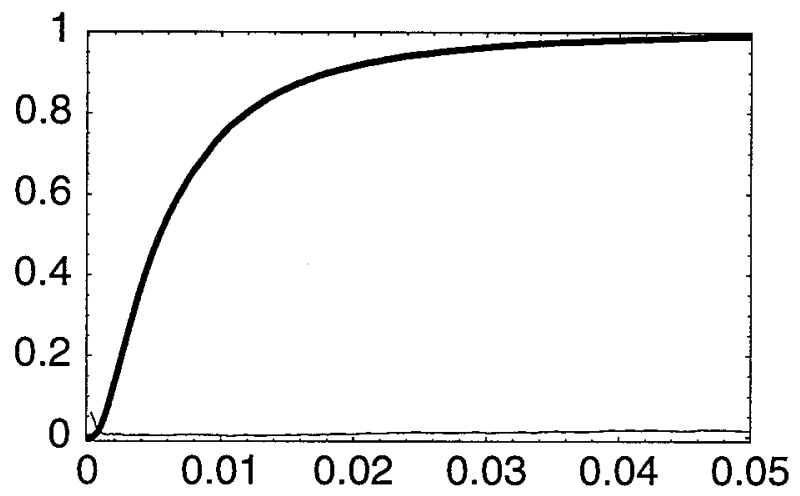

sdf = StandardizeCdf [cdf, 404]; EV [sdf, Empirical $\rightarrow$ True]

0.00835208

Put [sdf, PathName [ECinhaleAng] ];

ECinhaleAng $=$ Get $[$ PathName [ECinhaleAng $]$;

ECinhaleAngBar $=0.00835207927948548345^{\circ}$;

end

- ECinhaleBar = Uncertainty in Population-Average Level

$\{$ Conc $\}=$ uncertain variate

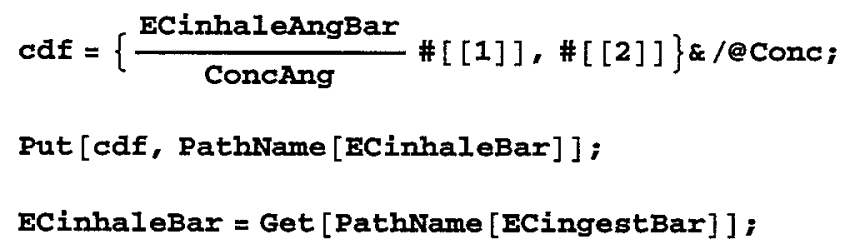

\section{Dermal Effective Dose (mg TCA/L plasma)}

\section{- <Edermal $>$ = Variability in Expected Level}

\{VolDist,BW,Vmet,Fmr,SABW,Fs,Kp,ETshower,T13\} = heterogeneous variates Correlation between Vmet $=\mathrm{Ve}$ and Fmr is assumed to be 0

Correlation between VolDist and BW is assumed to be -.5 


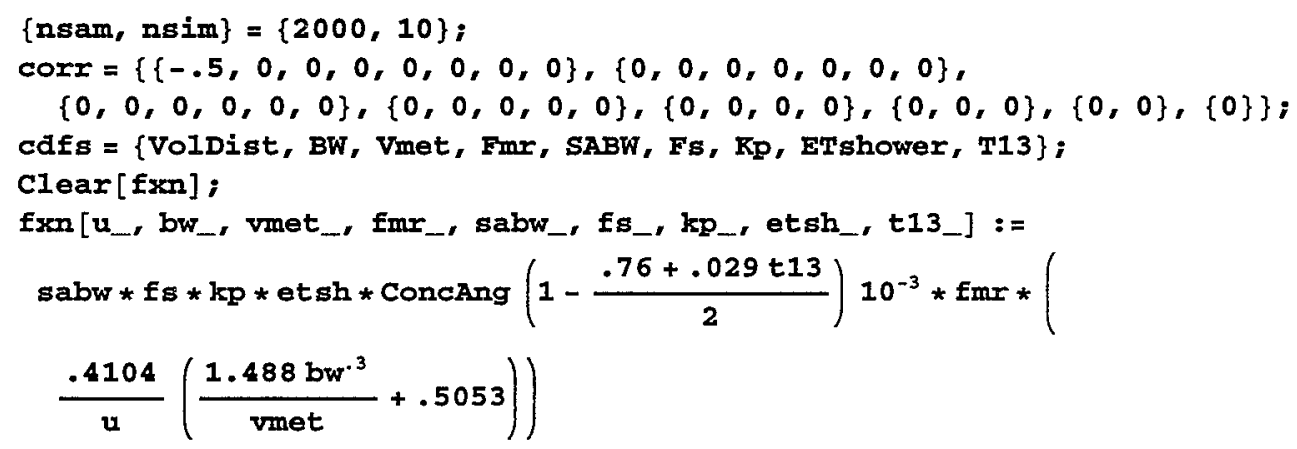

$\mathrm{fxn}[.1,70,1, .7, \mathrm{SABWBar}, .65, .263, .129,0]$

0.00166356

$(\operatorname{CdfQ}[\#]|| \mathrm{RQ}[\mathrm{Test}, \#[[1]], \#[[2]]]) \& / @ \mathrm{cdfs}$

\{True, True, True, True, True, True, True, True, True\}

Timing $[\{$ jen, cdf, cvm $\}=$ QUAnalyze [cdfs, fxn, nsam, nsim, Scale $\rightarrow 1$, Correlate $\rightarrow$ corr] $]$

$\{173.433$ Second, Nul1\}

TBL / jen

\begin{tabular}{|c|c|c|c|c|c|c|c|}
\hline$\left\{\begin{array}{l}\text { Mean }[\Delta r] \\
0.000459795\end{array}\right.$ & $\begin{array}{l}\operatorname{Max}[|\Delta r|] \\
0.0212962\end{array}$ & $\begin{array}{l}\text { JennrichChi2 } \\
4.44316\end{array}$ & $\begin{array}{l}\text { DegFr } \\
36\end{array}$ & $\begin{array}{l}\text { P-adj } \\
1 .\end{array}$ & $\begin{array}{l}\text { Fractile } \\
0.01 \\
0.05 \\
0.5 \\
0.95 \\
0.99 \\
\text { Mean }\end{array}$ & $\begin{array}{l}\text { Value } \\
0.000268902 \\
0.000445612 \\
0.00163022 \\
0.00625184 \\
0.0111429 \\
0.00228462\end{array}$ & $\begin{array}{l}\text { CVM (8) } \\
0.97206 \\
0.780994 \\
0.369624 \\
1.02696 \\
0.914124 \\
0.1228\end{array}$ \\
\hline & & & & & Variance & $5.0004 \times 10^{-6}$ & 2.93471 \\
\hline
\end{tabular}

PlotCdf $[\{c v m$, cdf $\}, Y \min \rightarrow-.01, X \min \rightarrow-.00001, X \max \rightarrow .01] ;$

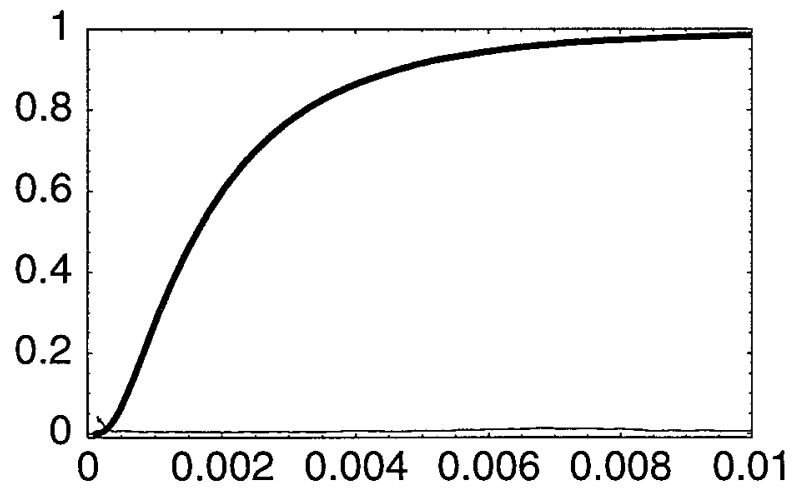

sdf = StandardizeCdf $[$ cdf, 404]; EV [sdf, Empirical $\rightarrow$ True $]$

0.00226194

Put [sdf, PathName [ECdermalAng] ];

ECdermalAng = Get $[$ PathName [ECdermalAng $]$;

ECdermalAngBar $=0.00226194038376105455^{\circ}$; 
end

\section{- EdermalBar $=$ Uncertainty in Population-Average Level}

$\{$ Conc $\}=$ uncertain variates

$$
\begin{aligned}
& \text { cdf }=\left\{\frac{\text { ECdermalAngBar }}{\text { ConcAng }} \#[[1]], \#[[2]]\right\} \& \text { / COAnc; } \\
& \text { Put }[\text { cdf, PathName }[\text { ECdermalBar }]] ; \\
& \text { ECdermaIBar }=\operatorname{Get}[\text { PathName }[\text { ECdermalBar }]] ;
\end{aligned}
$$

end

end 


\section{Appendix 3.F Effective Dose Correlations}

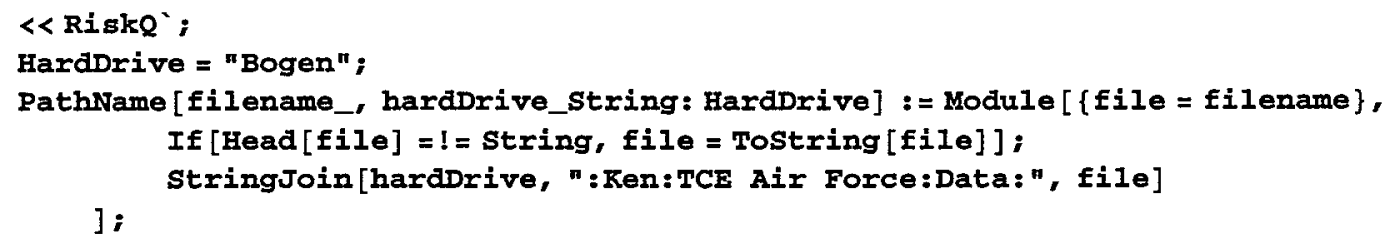

\section{Inputs}

\section{Log-Transform Utility Functions}

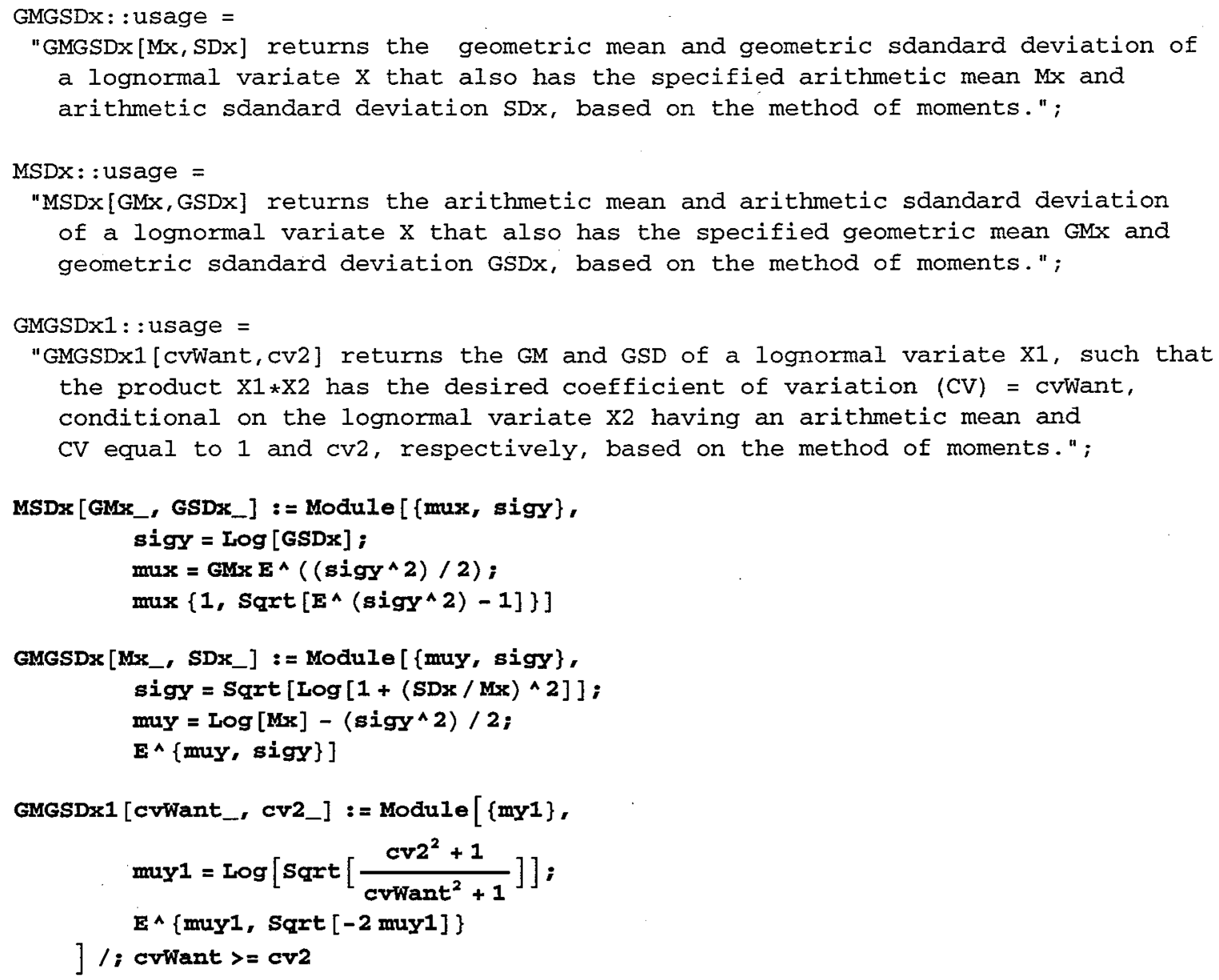




\section{- Input Empirical (Derived) Distributions}

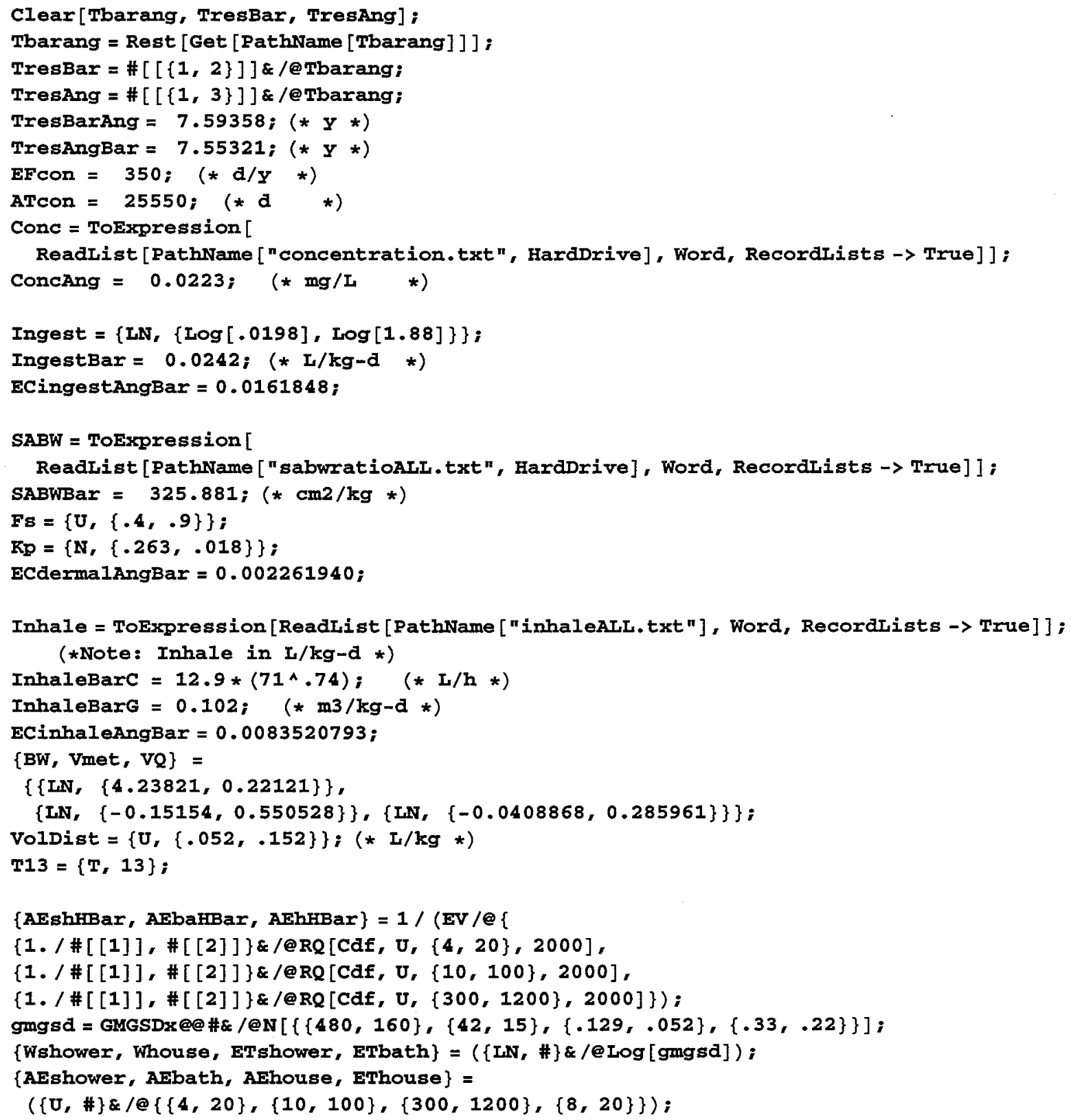




\section{- Fractions metabolized (summary-see Effective Cytotoxic Dose.nb)}

Correlation between VolDist and $\mathrm{BW}=-0.50$ (assumed approximation)

Correlation between $\mathrm{Ve}$ and $\mathrm{Fmo}=0$, and between $\mathrm{Ve}$ and $\mathrm{Fmr}=0$

Correlation between VQ and Fmo $=-0.43$

Correlation between VQ and Fmr $=-0.75$

Correlation between Fmr and Fmo $=0.83$

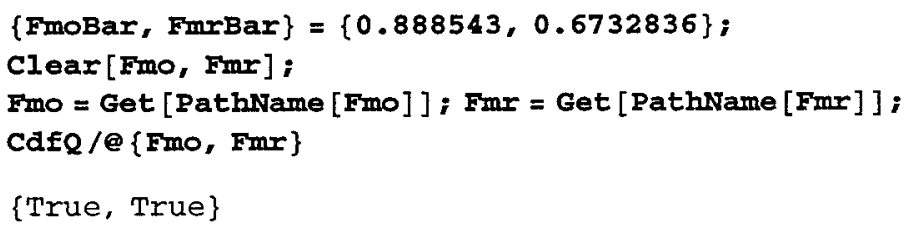

end

end

\section{Effective Dose Uncertainty}

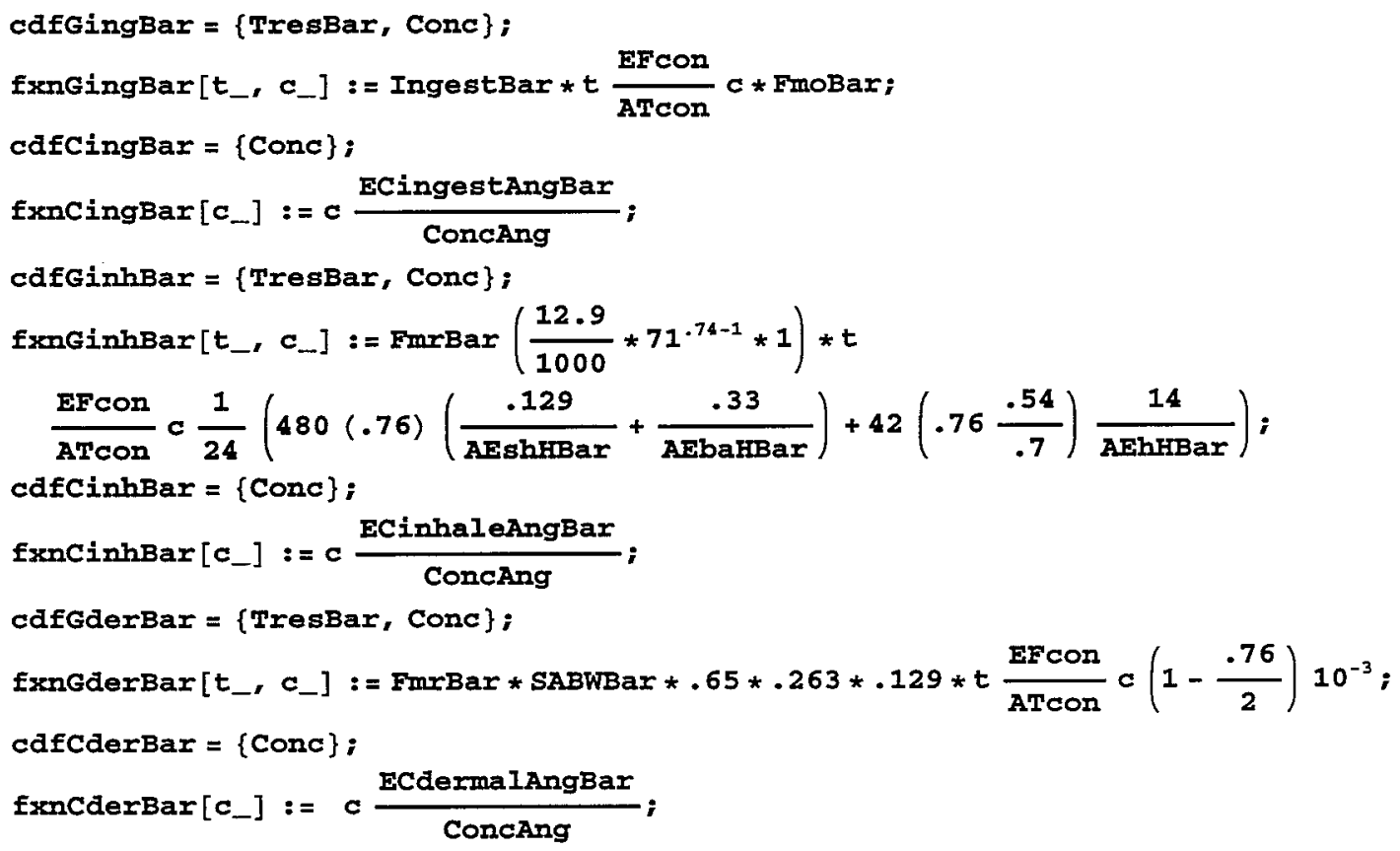

All 6 functions above are linear functions of either $\mathrm{c}$ or $\mathrm{c} * \mathrm{t}$; thus: all those involving just $\mathrm{c}$ are $100 \%$ correlated, all those involving just $c^{*} \mathrm{t}$ are $100 \%$ correlated, and correlations between those involving $\mathrm{c}$ vs. $\mathrm{c} * \mathrm{t}$ are given by: 


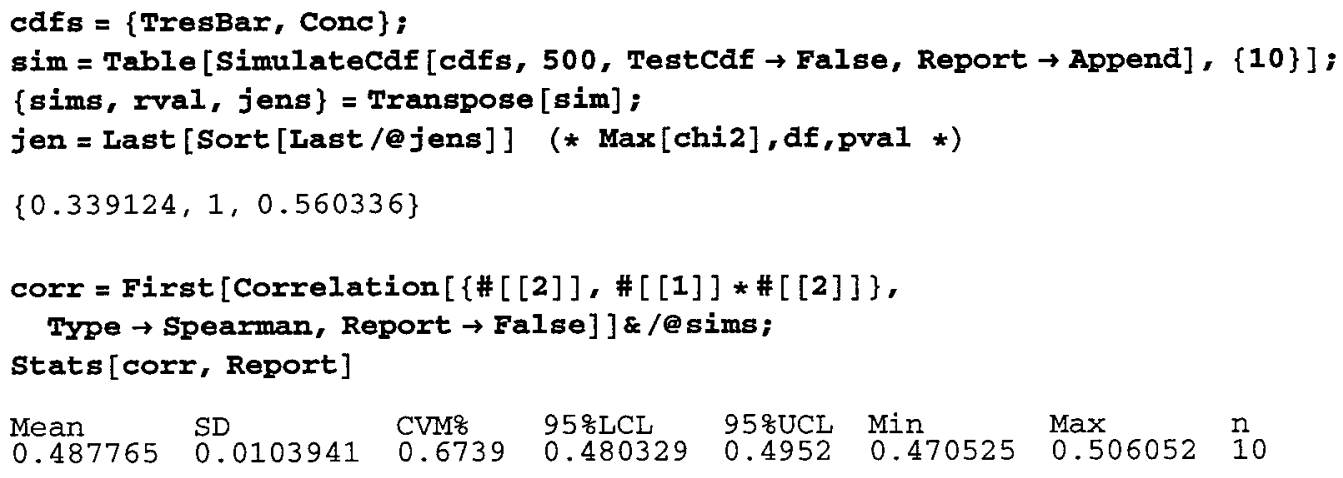




\section{Effective Dose Variability}

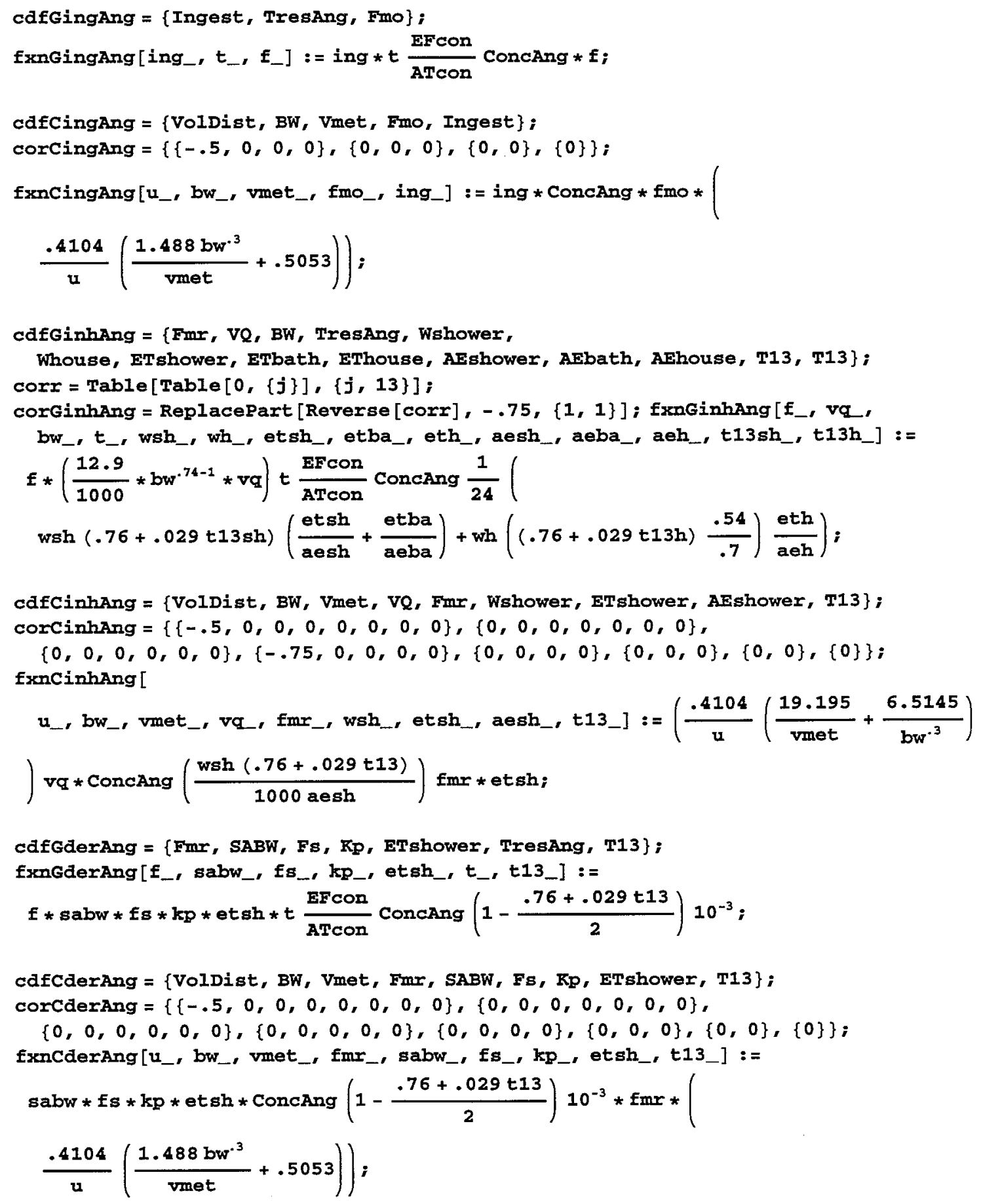

Function \{argument positions\}:

fxnGingAng[ing_,t_,f_]

$\{7,8,5\}$

fxnCingAng[u_,bw_,vmet_,fmo_,ing_]

$\{1,2,3,5,7\}$ 
fxnGinhAng[f_,vq_,bw_t_,wsh_,wh_,etsh_etba_eth_,aesh_,aeba_,aeh_,t13sh_,t13h_]

$\{6,4,2,8,9,10,11,12,13,14,15,16,17,18\}$

fxnCinhAng[u_,bw_,vmet_,vq_,fmr_,wsh_,etsh_,aesh_,t13_]

$\{1,2,3,4,6,9,11,14,17\}$

fxnGderAng[f_sabw_,fs_,kp_,etsh_,t,t13_]

$\{6,19,20,21,11,8,17\}$

fxnCderAng[u_,bw_,vmet_,fmr_,sabw_,fs_,kp_,etsh_,t13_]

$\{1,2,3,6,19,20,21,11,17\}$

cdfs = Voldist, BW, Vmet, VQ, Fmo, Fmr, Ingest, TresAng,
Wshower, Whouse, ETshower, ETbath, EThouse, AEshower,
AEbath, AEhouse, T13, T13, SABW, Fs, Kp $\}$ ( $\mathrm{n}=21 *)$

$(\operatorname{CdfQ}[\#]|| \operatorname{RQ}[$ Test, \#[[1]], \#[[2] ] $) \& / @ \operatorname{cdfs}$

\{True, True, True, True, True, True, True, True, True, True, True, True, True, True, True, True, True, True, True, True, True

Correlation between VolDist and BW $=-0.50$ (assumed approximation)

Correlation between $\mathrm{Ve}$ and $\mathrm{Fmo}=0$, and between $\mathrm{Ve}$ and $\mathrm{Fmr}=0$

Correlation between VQ and Fmo $=-0.43$

Correlation between VQ and $\mathrm{Fmr}=-0.75$

Correlation between Fmr and Fmo $=0.83$

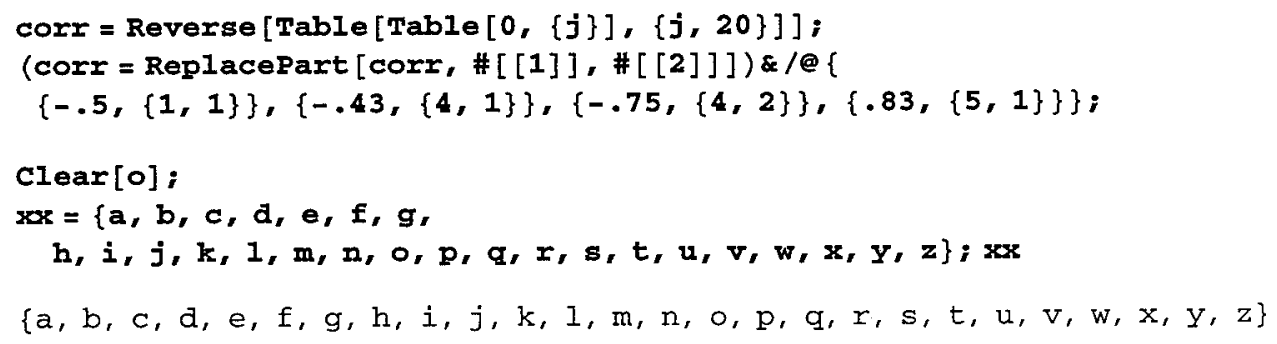

Define fxns here each as a function of elements of the convenient dummy variate $\mathrm{xx}$ :

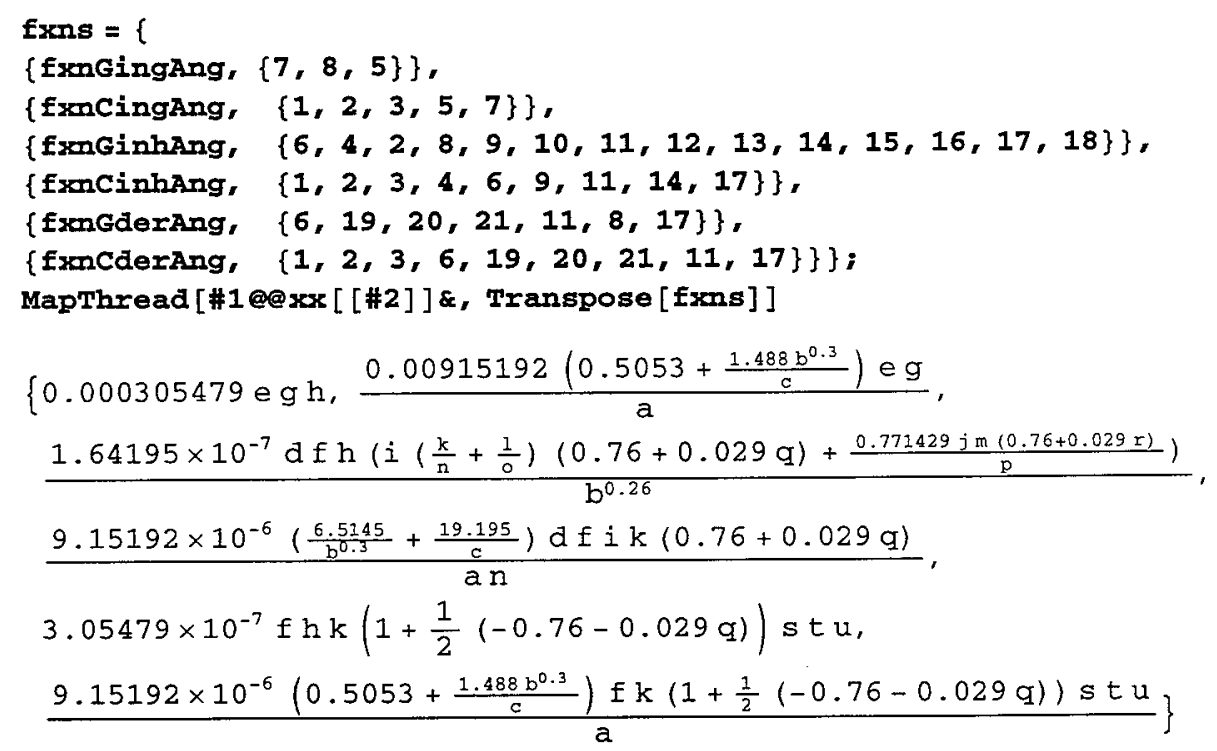


Select a series of 50 simulated-variate sets that each have a 21 -variate correlation matrix (=corr, defined above) that is not significantly different than corr. Below, 20 of a total of 70 sets tried qualify for use using a p-value of $<0.01$ to reject:

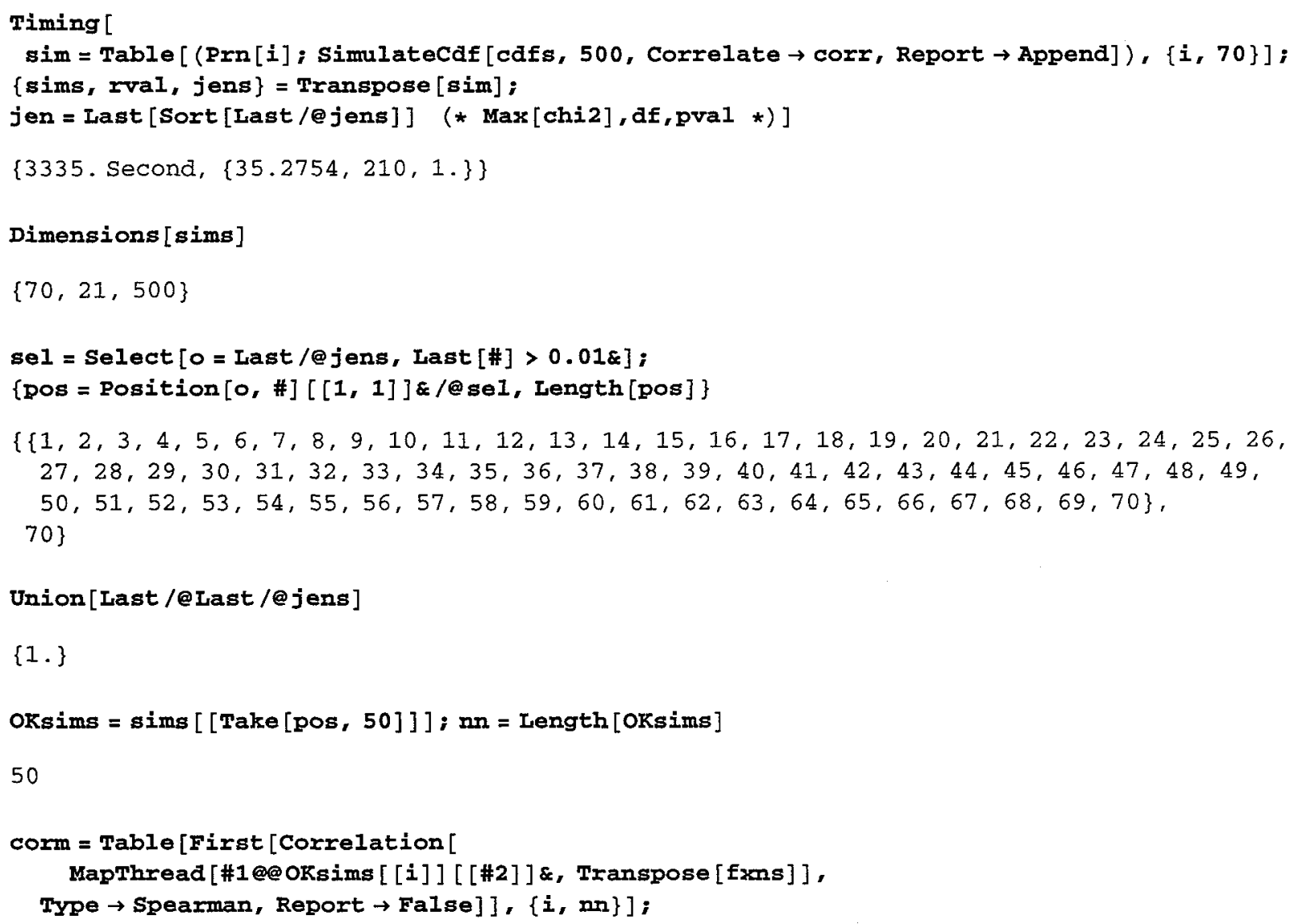

Mean corr, SDM[corr], and CVM[corr] values for:

\section{GingAng CingAng GinhAng CinhAng GderAng CderAng}

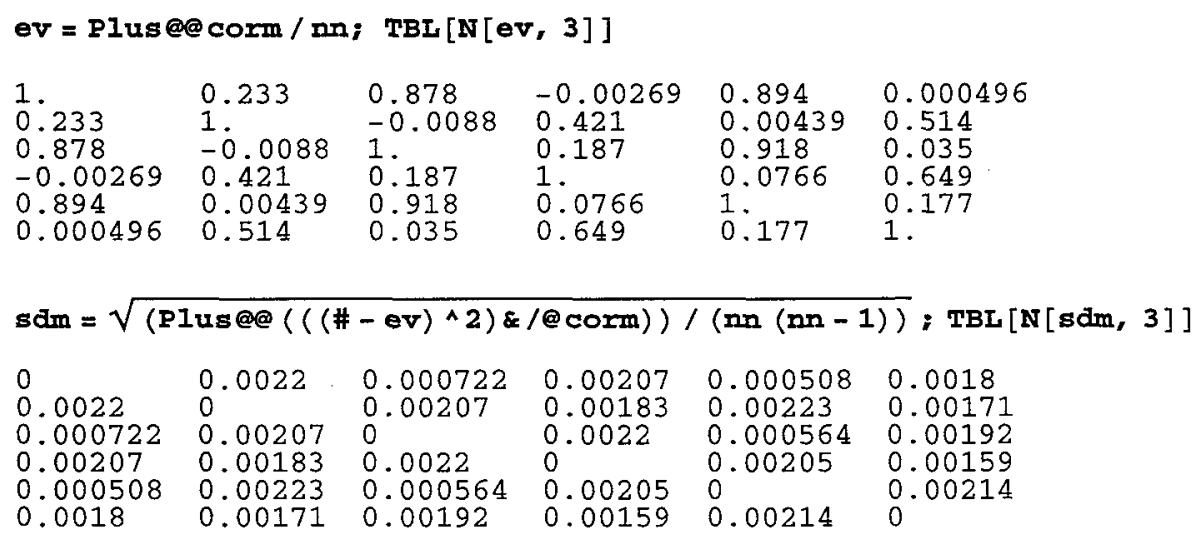


$\operatorname{cvm}=\mathrm{Abs}[100 \mathrm{sdm} / \mathrm{ev}] ; \operatorname{TBL}[\mathrm{N}[\mathrm{cvm}, 3]]$

$\begin{array}{llllll}0 & 0.946 & 0.0822 & 76.7 & 0.0568 & 364 .\end{array}$

$\begin{array}{llllll}0.946 & 0 & 23.5 & 0.435 & 50.8 & 0.333 \\ 0.0822 & 23.5 & 0 & 1.17 & 0.0614 & 5.49\end{array}$

$\begin{array}{llllll}76.7 & 0.435 & 1.17 & 0 & 2.68 & 0.245\end{array}$

$\begin{array}{llllll}0.0568 & 50.8 & 0.0614 & 2.68 & 0 & 1.21 \\ 364 . & 0.333 & 5.49 & 0.245 & 1.21 & 0\end{array}$

end 


\section{Appendix 3.G}

\section{Potency}

$<<$ RiskQ;

< Minimize;

\section{Multistage (Genotoxicity) Model}

\section{- Multistage Potencies for TCE Cancer Bioassays}

The Mathematica program "QFit" (by K.T. Bogen, LLNL—see "RiskQ Functions Used" section below) was used to obtain for each bioassay data set a distribution reflecting parameter-estimation uncertainty pertaining to the value of multistagemodel "potency" (denoted $q_{1}$ ), that is, the value of the linear coefficient of dose $D$ in the multistage model of cancer risk, which posits that cancer risk is essentially an exponentiated-polynomial function dose. Conditional on any sufficiently "upper-bound" (i.e., conservative) estimate (denoted $q_{1}{ }^{*}>0$ ) of the linear "potency" term $\left(q_{1}\right)$, the multistage model guarantees that any small increase in cancer risk will be very nearly equal to the product: $q_{1}{ }^{*} \times D$. Uncertainty distributions are derived corresponding to each of seven bioassay data sets considered below; one data set (data set \#7 below concerning the study by Henchler et al., 1980, showing malignant lymphoma in female HAN:NMRI mice) is excluded for reasons noted below.

\section{- 1. NCI 1976 Mouse B6C3F1: M 34 g HCC}

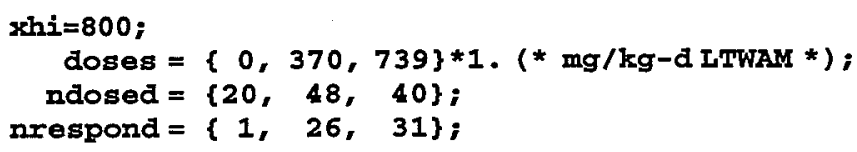


GTCE1=QFit [doses, ndosed, nrespond, 500, PolyDegree->2, Exponentiated->True,

ConfInterval->.90, Output->01,

$x \min ->-x h i / 100, x \max ->1.01 * x h i, Y \min ->0, Y \max ->1]$;

The Optimized Function $F$ of Dose $d$ is:

$F(d)=1-\exp [-P(d)]$, where:

$P(d)=0.0524116+0.00199086 d+2.72013 \times 10^{-8} d^{2}$

Chisquare $(1)=0.032011 \quad 1$-tailed $p=0.858003$

$R^{2}=0.999488$

F(d) vs Data

(\& Bootstrap 908 Conf. Limits on Data)

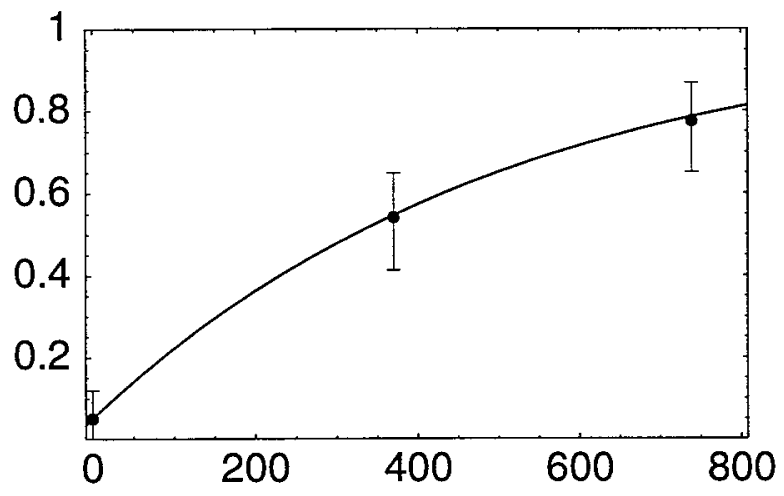

\section{- 2. NCI 1976 Mouse B6C3F1: F $29 \mathrm{~g}$ HCC}

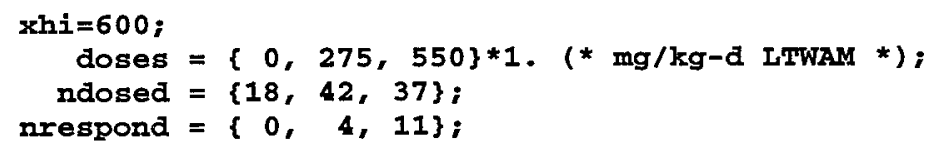


qTCE2=QFit [doses, ndosed, nrespond, 500,

PolyDegree->2, Exponentiated->True,

ConfInterval $>.90$, Output- $>Q 1$,

$x \min ->-x h i / 100, X \max ->1.01 * x h i, Y \min ->0, Y \max ->1]$;

The Optimized Function $\mathrm{F}$ of Dose d is:

$F(d)=1-\exp [-P(d)]$, where:

$P(d)=0.0000863863 d+1.00929 \times 10^{-6} d^{2}$

Chisquare(1) $=0$. Perfect fit.

$\mathrm{R}^{2}=1$

\section{F(d) vs Data}

( $\&$ Bootstrap $90 \%$ Conf. Limits on Data)

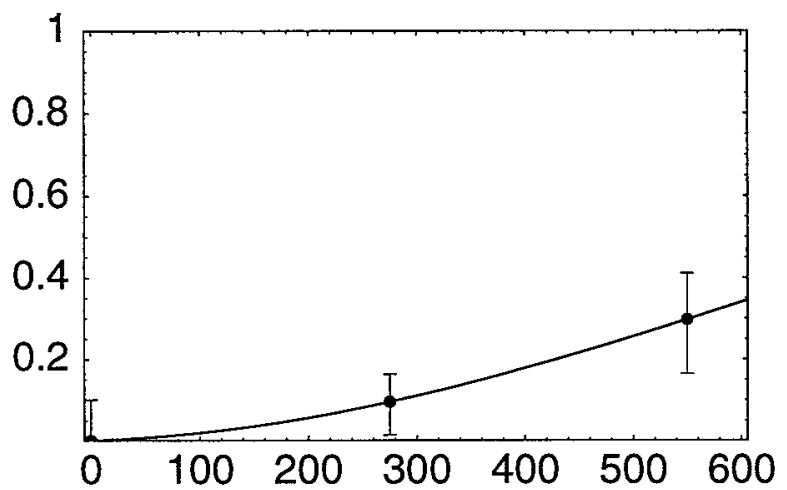

- 3. NTP 1983 Mouse B6C3F1: M 37 g HCC or HCA

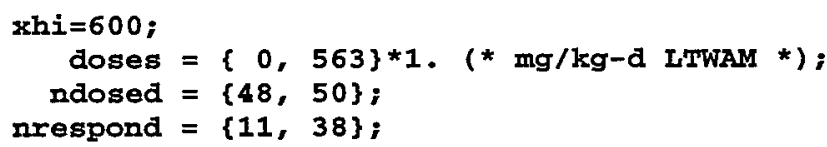


GTCE3=QFit [doses, ndosed, nrespond, 500,

PolyDegree->1, Exponentiated->True,

ConfInterval->.90, Output->01,

$x \min ->-x h i / 100, x \max ->1.01 * x h i, Y \min ->0, Y \max ->1]$;

The Optimized Function $F$ of Dose d is:

$F(d)=1-\exp [-P(d)]$, where:

$P(d)=0.260283+0.00207253 d$

Chisquare $(0)=0$. Perfect fit.

$\mathrm{R}^{2}=1$

F(d) vs Data

(\& Bootstrap $90 \%$ Conf. Limits on Data)

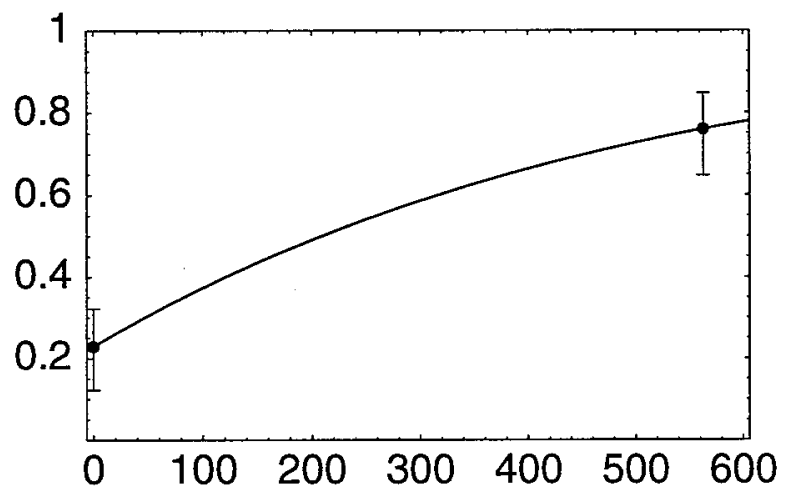

\section{- 4. NTP 1983 Mouse B6C3F1: F 33 g HCC or HCA:}

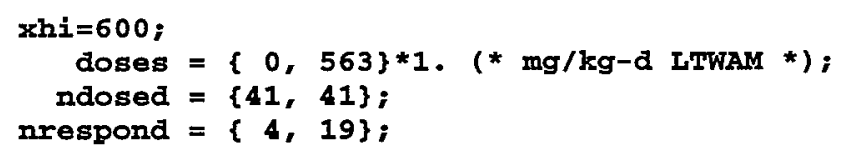


GTCE4=QFit [doses, ndosed, nrespond, 500,

PolyDegree->1, Exponentiated->True,

ConfInterval->.90, Output- $>01$,

$x \min ->-x h i / 100, x \max ->1.01 * x h i, Y \min ->0, Y \max ->1]$;

The Optimized Function F of Dose d is:

$F(d)=1-\exp [-P(d)]$, where:

$P(d)=0.102654+0.000923402 d$

Chisquare $(0)=0$. Perfect fit.

$\mathrm{R}^{2}=1$

$F(d)$ vs Data

( $\&$ Bootstrap 908 Conf. Limits on Data)

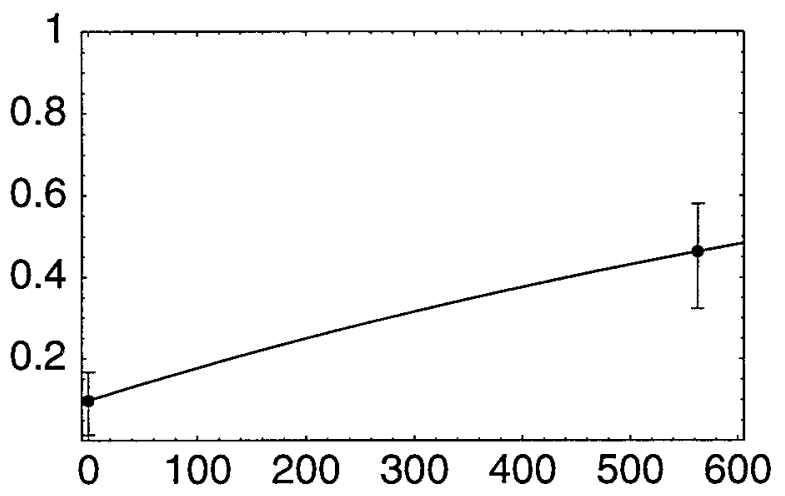

- 5. NTP 1983 Rat F344/N: M 340 g RenalTub Adenocarcinoma

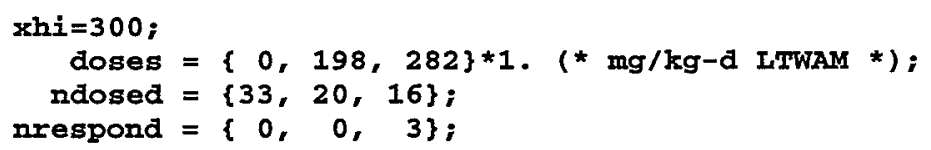




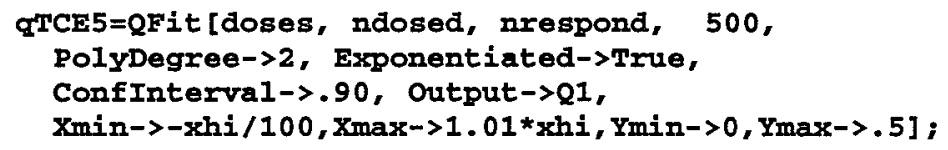

$$
F(\bar{\alpha}) \text { vs Data }
$$

(\& Bootstrap 908 Conf. Limits on Data)

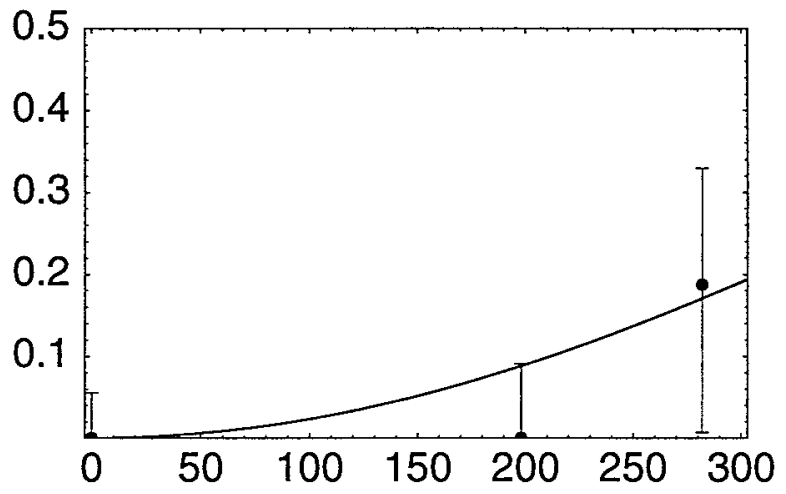

\section{- 6. Bell et al. 1978 Mouse B6C3F1: M 35(?) g HCC or HCA}

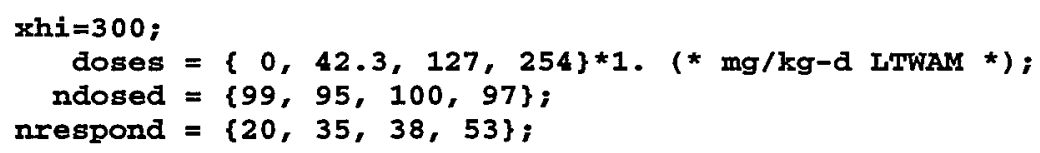




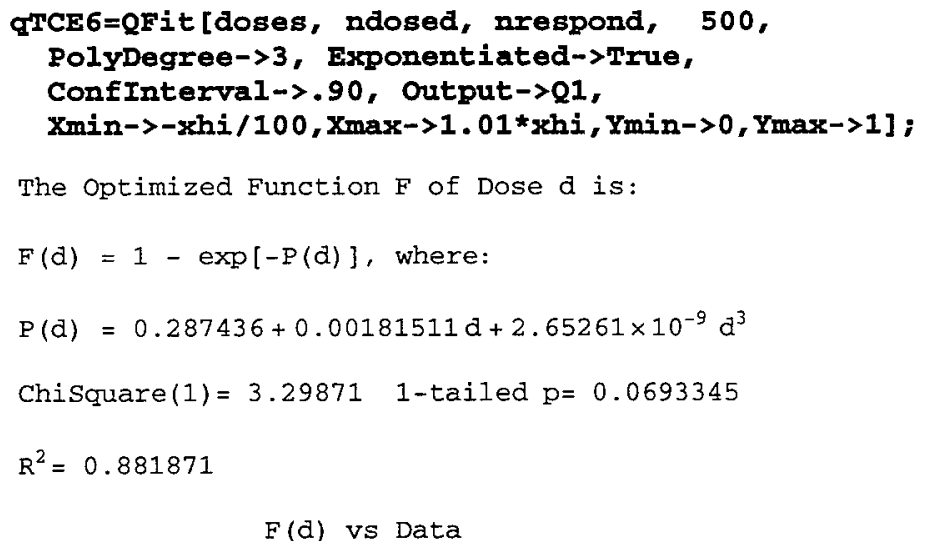

(\& Bootstrap $90 \%$ Conf. Limits on Data)

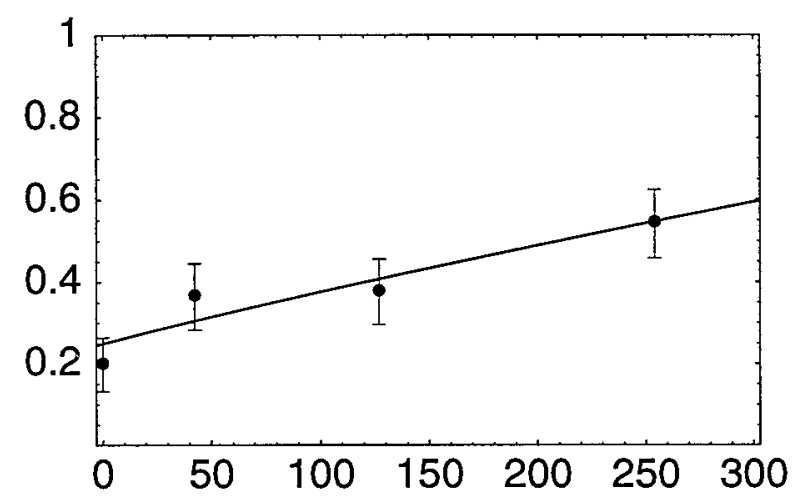

\section{- 7. Henchler et al. 1980 Mouse Han:NMRI: F 30(?) g Malig. Lymphoma}

Henchler did not consider this positive--called the study negative;

High spontaneous Malig. Lymphoma incidence is peculiar to this strain of mice in females (inborn murine lymphoma virus)

$p=0.03$ by Fisher Exact for females (this data set) $p=1$ for males

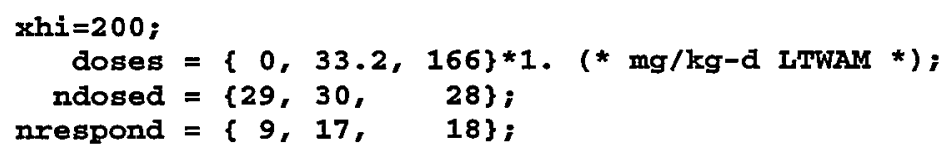




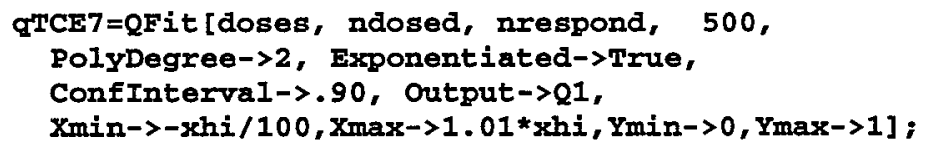

F(d) vs Data

(\& Bootstrap $90 \%$ Conf. Limits on Data)

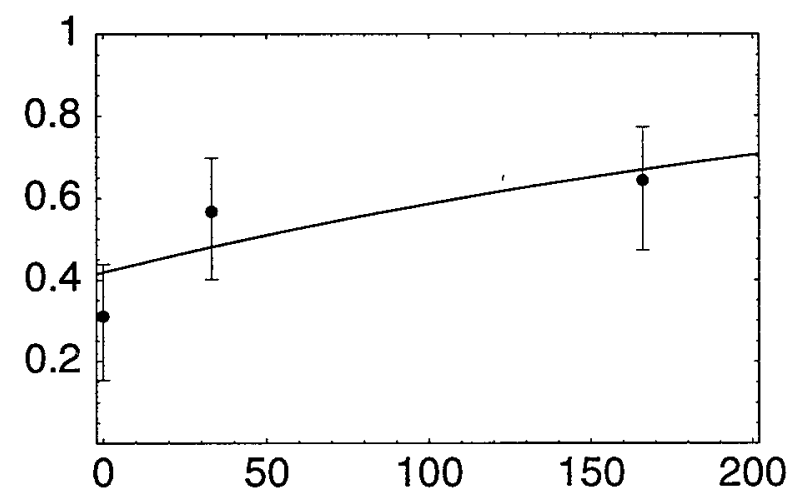

- 8. Maltoni et al. 1986 Mouse Swiss: F 30(?) g Malig. Hepatoma

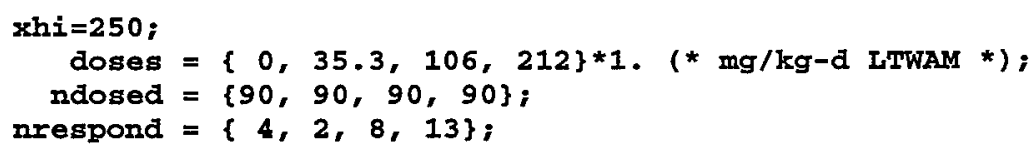


qTCE $8=Q F i t$ [doses, ndosed, nrespond, 500,

PolyDegree->3, Exponentiated->True,

ConfInterval->.90, Output->Q1,

$x \min ->-x \operatorname{xh} i / 100, X \max ->1.01 * x h i, Y \min ->0, Y \max ->.25]$;

The Optimized Function $F$ of Dose $d$ is:

$F(d)=1-\exp [-P(d)]$, where:

$P(d)=0.0329504+0.000306602 d+1.36839 \times 10^{-6} d^{2}$

ChiSquare $(1)=1.62806 \quad 1$-tailed $p=0.201971$

$R^{2}=0.911347$

F (d) vs Data

(\& Bootstrap $90 \%$ Conf. Limits on Data)

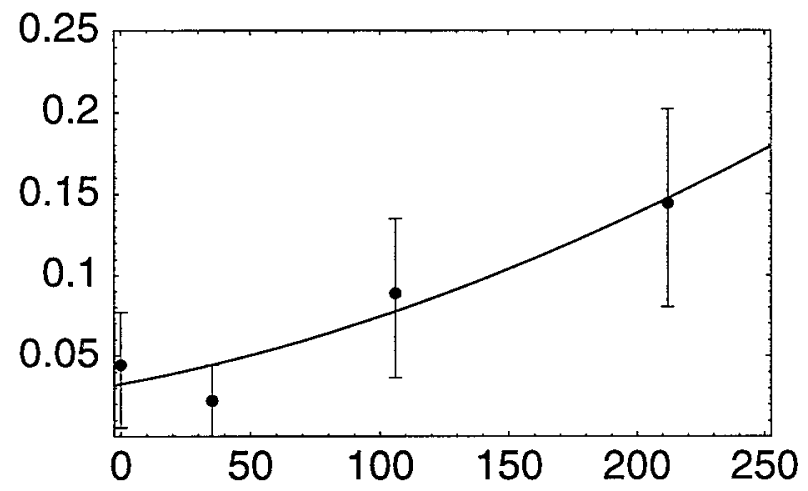

qal1 = qTCE1, qTCE2, qTCE3, qTCE4, qTCE5, qTCE6, GTCE7, qTCE 8\};

\section{Weighted-Average TCE Cancer Potency}

- Define qall

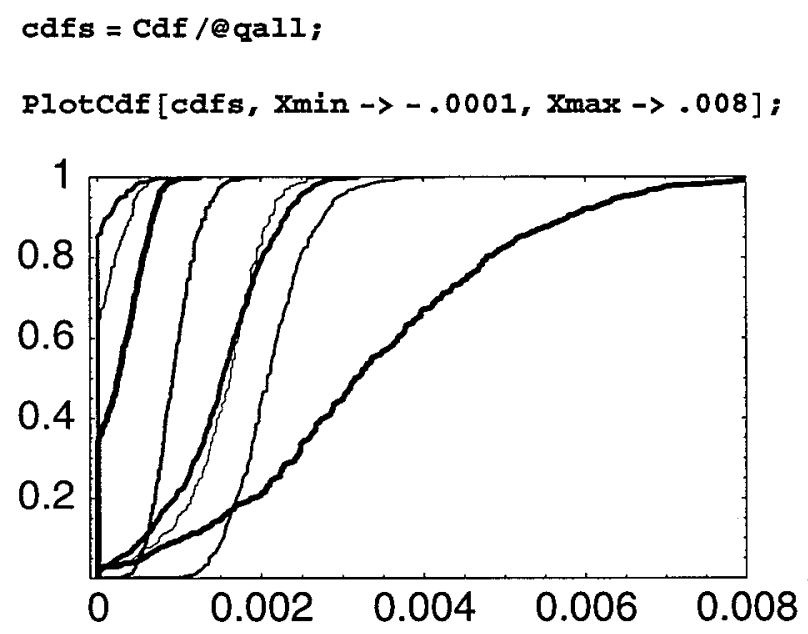


Remove Henchler lymphoma data, due to his determination that this was a negative study given the likelihood of murine lymphoma virus involvement:

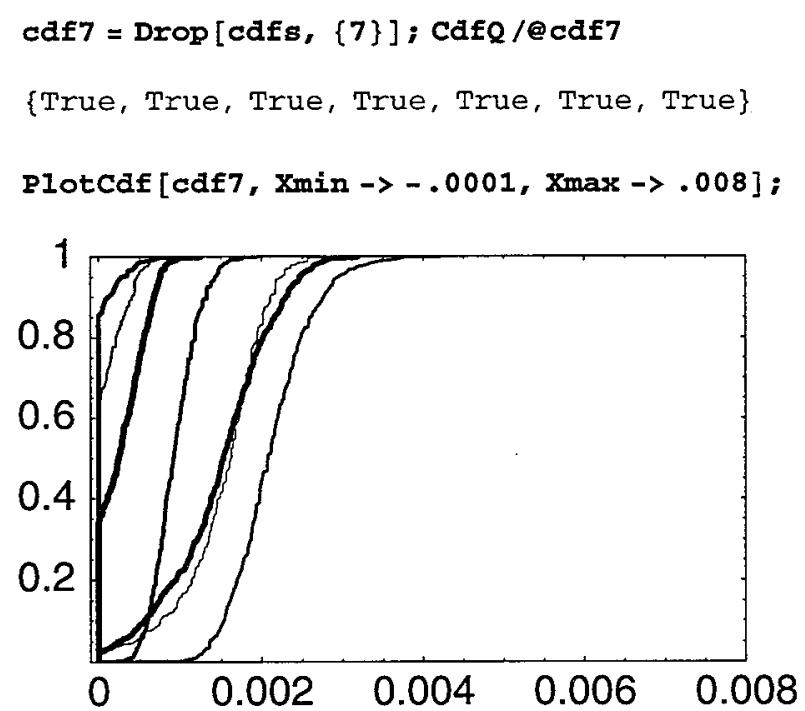

Replot to look nicer:

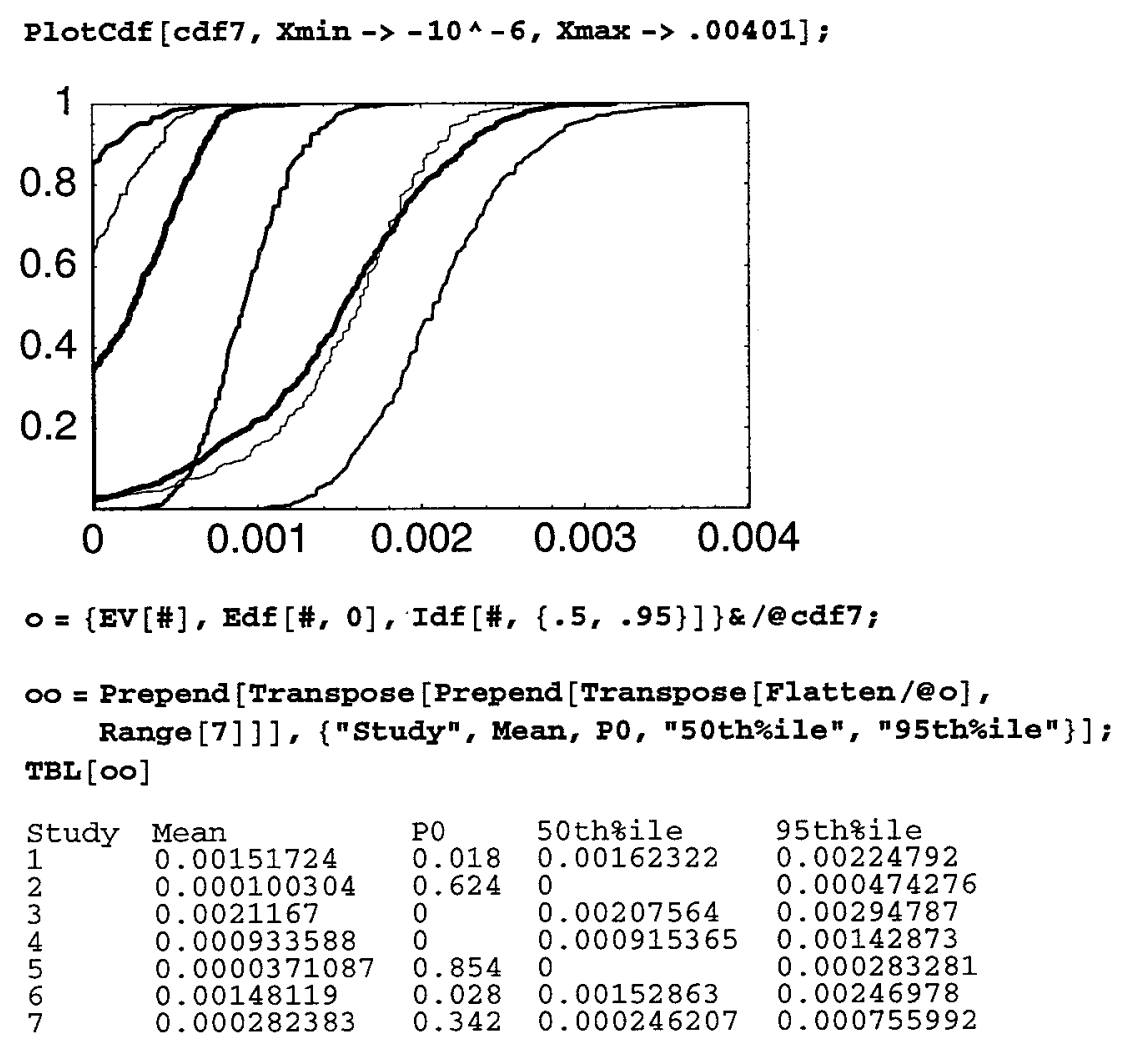

\section{Study-Weighting Logic:}

\{Species, Strain, Sex, Site, Study\}-specific data are equally likely, therefore:

Data sets $\{\{\{\{1,3,6\},\{2,4\}\}, 8\}, 5\}=\{$ mouse,rat $\}=\{$ mouse $\{$ b $6 \mathrm{c} 3 \mathrm{f} 1\{\mathrm{~m}, \mathrm{f}\}$,swiss $\}$,rat $\}$

get relative weights: $\{1,1\}=\{\{\{\{1,1,1\},\{1.5,1.5\}\}, 6\}, 12\}$ 
(Note that Henchler lymphoma data was removed, due to his determination that this was a negative study given the likelihood of murine lymphoma virus involvement.)

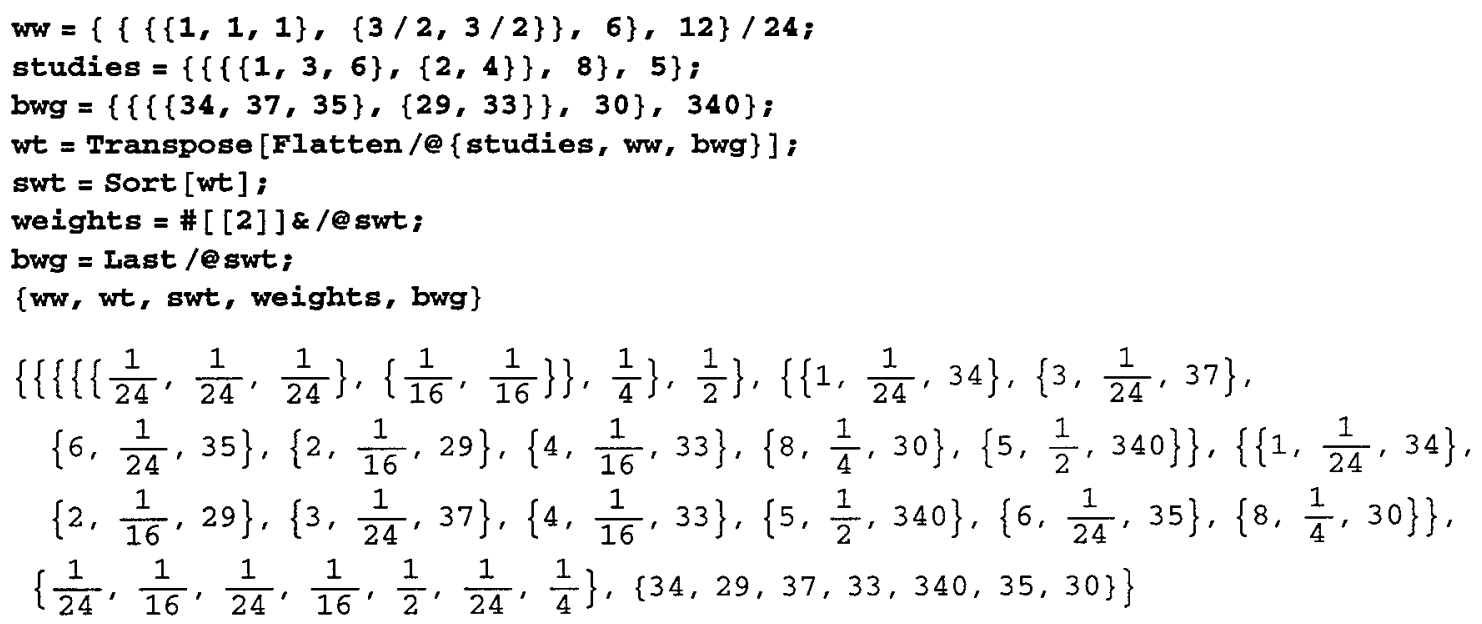

Average the study-specific cdfs using the study-specific weights ("weights") defined above, plot the results, and get statistics for the resulting averaged cdf ("adfBW") based on a body-weight (i.e., using a mass-per-kg-body-weight) approach to interspecies scaling of equitoxic doses.

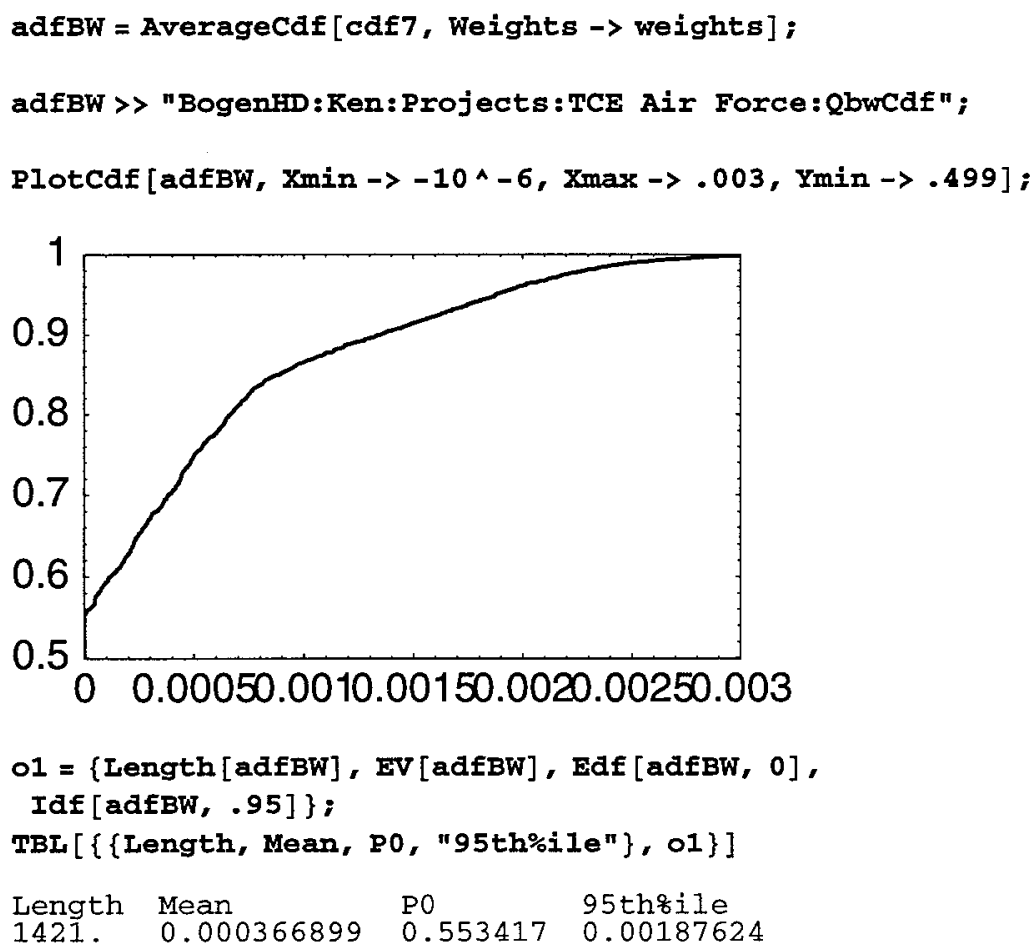

Multiply the abscissa of each cdf by $(\mathrm{Wh} / \mathrm{BW})^{\wedge} .25$, where $\mathrm{Wh}=70 \mathrm{~kg}$ and $\mathrm{BW}$ is rodent body weight in grams, i.e., scale using a BW. ${ }^{75}$ scaling factor. Then re-average the cdfs using the same study-specific weights as used above, to obtain the resulting averaged cdf ("adf75") based on a (body weight) ${ }^{75}$ (i.e., using a mass-per-(kg body weight) ${ }^{75}$ ) approach to interspecies scaling of equitoxic doses. 


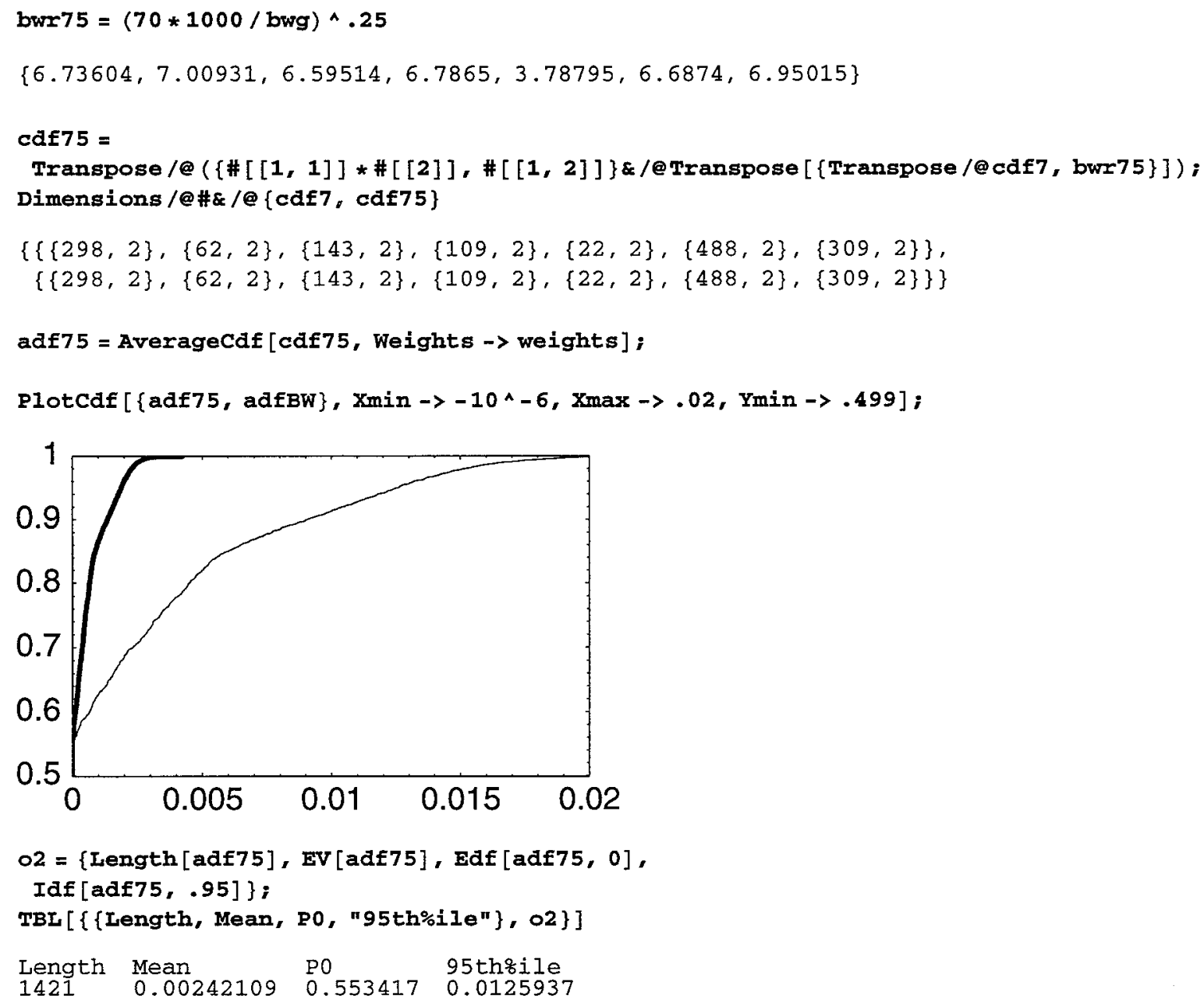

Average the cdfs "adfBW" and "adf75" assuming equal likelihood, standardize, simplify, plot, get statistics, and save:

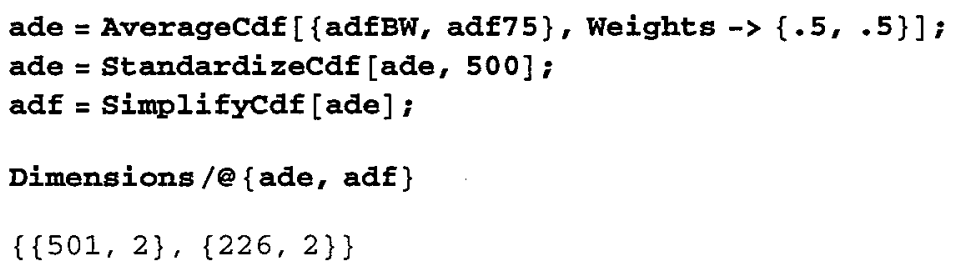


PlotCdf $[\{\operatorname{adf75}, \operatorname{adfBW}, \operatorname{adf}\}, \operatorname{xmin} \rightarrow-.00002, \mathrm{Xmax} \rightarrow .02, \operatorname{Ymin} \rightarrow .49]$;

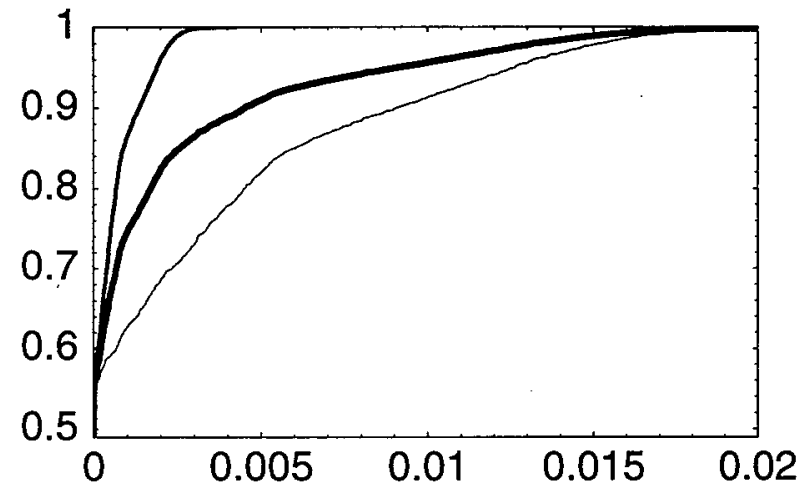

$012=\{$ Length [adf], EV [adf], Edf [adf, 0], Idf [adf, .95]\};

TBL $[\{$ Iength, Mean, PO, "95th\%ile" $\}, 012\}]$

$\begin{array}{llll}\text { Length } & \text { Mean } & \text { Po } & 95 \text { thrile } \\ 228 & 0.00140008 & 0.552 & 0.00902774\end{array}$

- Define adf

WriteMatrix ["BogenHD:Ken:Projects:TCE Air Force:Qcdf.txt", N[adf] ];

\section{TCE Threshold (Cytotoxicity) Model}

\section{TBARS dose-response in male B6C3F1 mice}

27-g male B6C3F1 mice (Larson \& Bull, 1992)

Dose in mg TCA per kg BW, vs. TBARS in nmol malondialdehyde equiv./g liver ( $\mathrm{n}=4$ @ ea. dose)

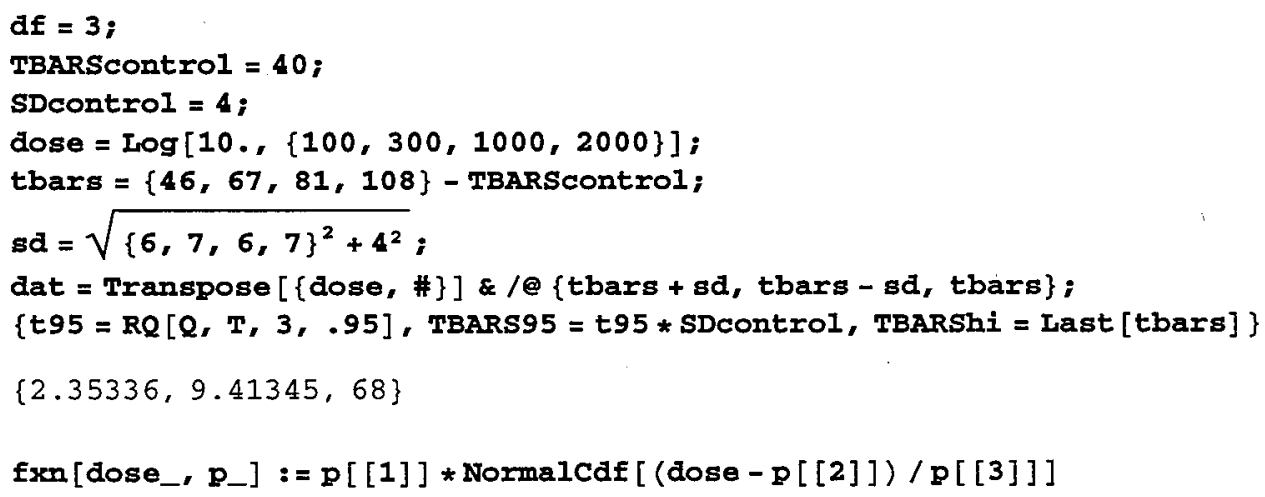

List "o" $=\left\{\right.$ parameter estimates, corresponding SE values, $\left\{\chi^{2}, \mathrm{df}, \mathrm{p}\right.$-value $\left.\}\right\}$ : 


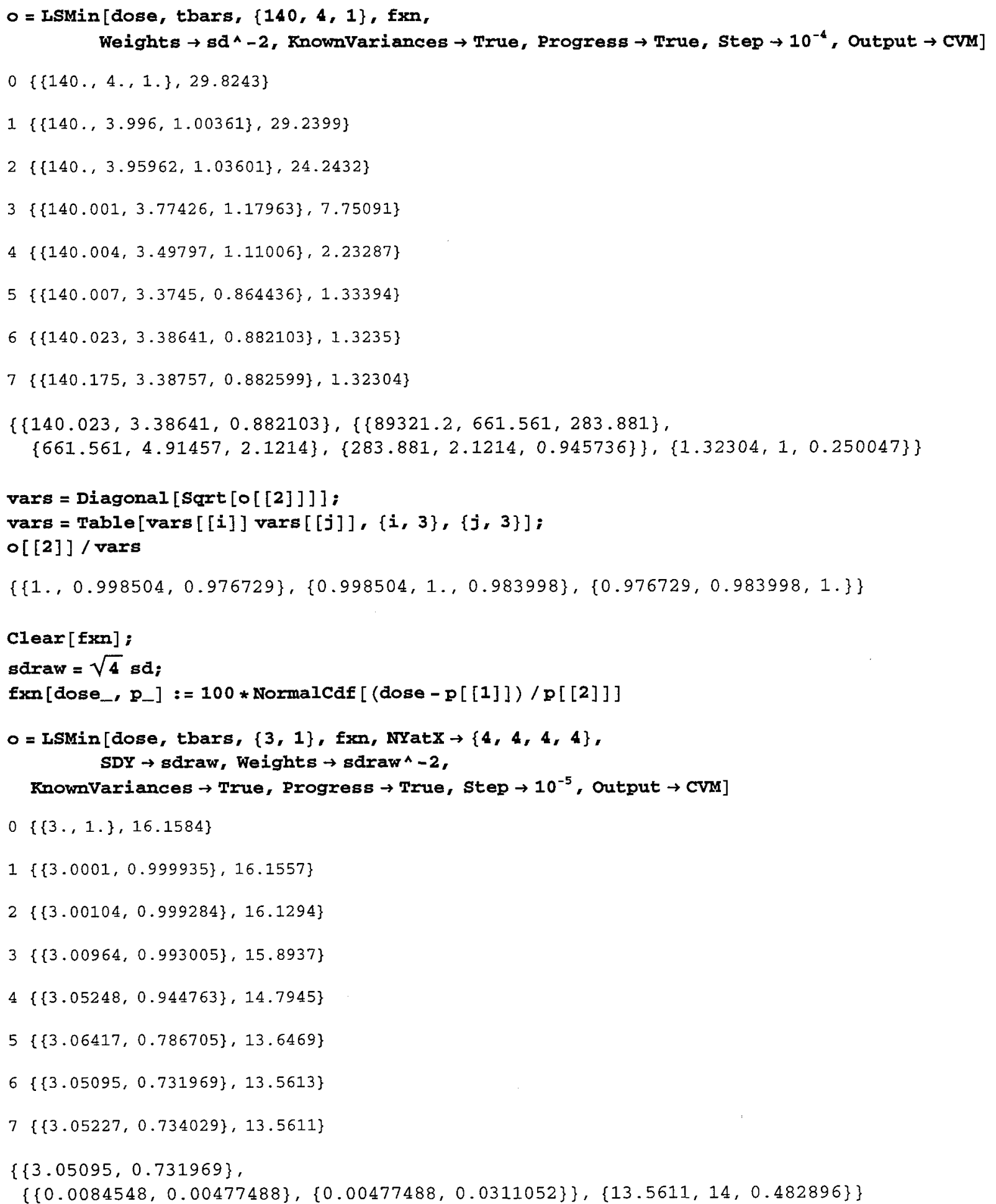

Calculate the Pearson product-moment correlation of the two fitted lognormal-model parameters: 


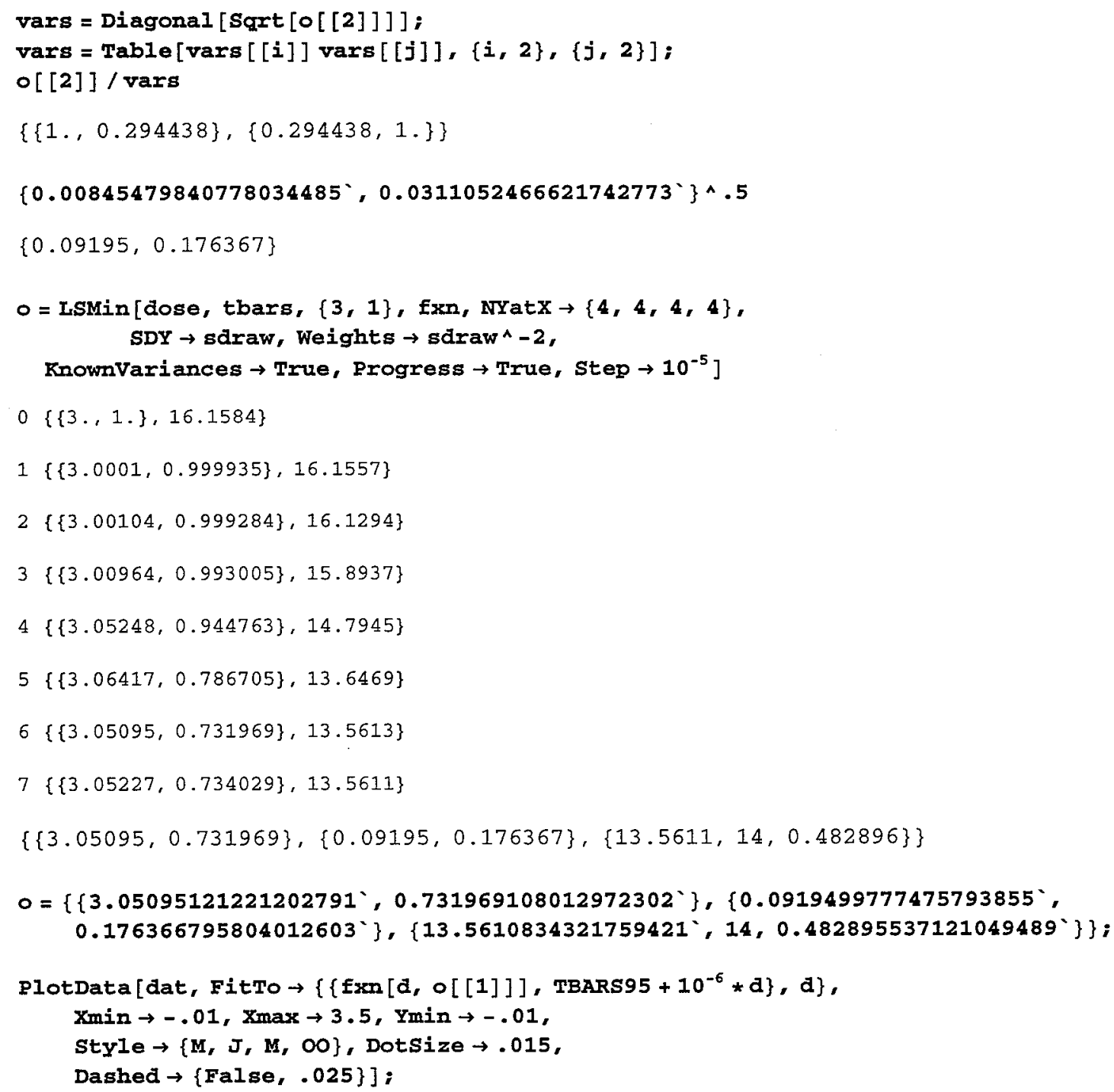

end 


\section{- Effective acute TCA threshold dose (mg/kg) for TBARS elevation}

\section{- Define cdfD95 = Prob\{significant TBARS elevation I Effective TCA dose (mg/L plasma)}

(with abscissa units of $\mathrm{mg}$ TCA/L plasma based on Larson \& Bull (TAP 115:268-277, 1992) using:

$\mathrm{Vd}=15.0 \mathrm{~mL}$

$\mathrm{Cmax}=790 \mathrm{nmol} \mathrm{TCA} / \mathrm{mL}$ plasma $=129.1 \mathrm{mg} \mathrm{TCA} / \mathrm{mL}$ plasma

$100 \mathrm{mg}$ TCA in water administered by gavage)

To do this, first analytically solve for values of $\mathrm{d} 95 \mid \mathrm{fxn}[\{\mathrm{p} 1, \mathrm{p} 2\}, \mathrm{d} 95]=$ TBARS95, where $\mathrm{d} 95$ is dose on a $\log 10(\mathrm{mg} / \mathrm{kg})$ scale, and fxn is the log-normal response function fitted above:

$100 *$ NormalCdf[(dose-p1)/p2],

where $\mathrm{p} 1$ and $\mathrm{p} 2$ and their estimated standard deviations are repeated below, and where errors in $\mathrm{p} 1$ and $\mathrm{p} 2$ are assumed to have a joint $\mathrm{T}$ distribution with $\mathrm{df}=14$ a correlation $\mathrm{r}$ equal to $\sim 0.294$.

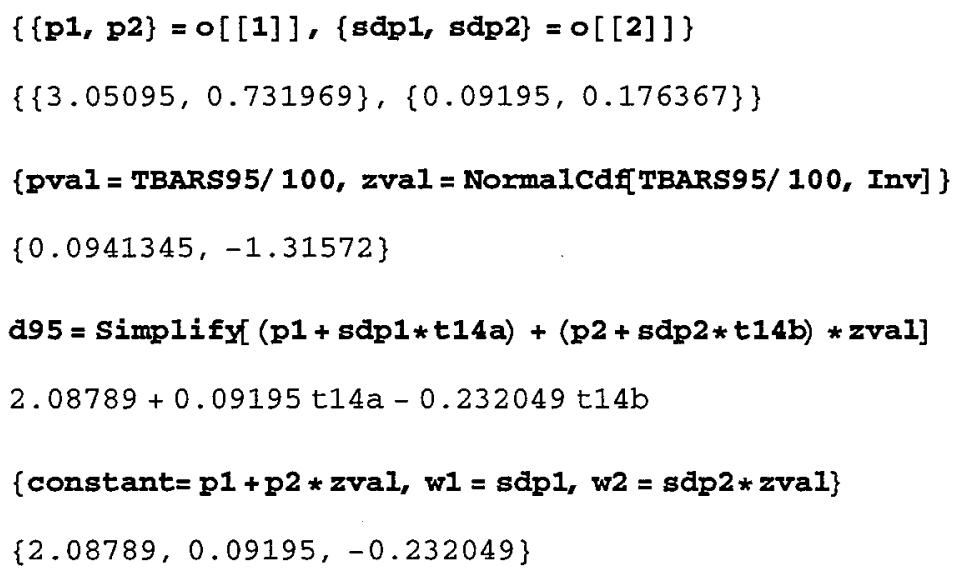

Therefore, $\mathrm{d} 95$ is distributed as a constant plus a weighted sum $(v)$ of two correlated T-ditributed variates having weights w1 and $\mathrm{w} 2$ and degrees of freedom $\mathrm{f} 1=14$ and $\mathrm{f} 2=14$, respectively. A numerical-analytic solution for the cumulative distribution function $\psi(v)$ of the weighted sum $(v)$ of two correlated T-variates is as follows, based on a straightforward extension of previously published results concerning independent weighted T-variates, to the case of correlated T-variates (Ruben, $\mathrm{H}$. 1960. On the distribution of the weighted sum of two independent Student variables. J. Royal Soc. Stat. Ser. B (Methodol.) 22, 188-194.):

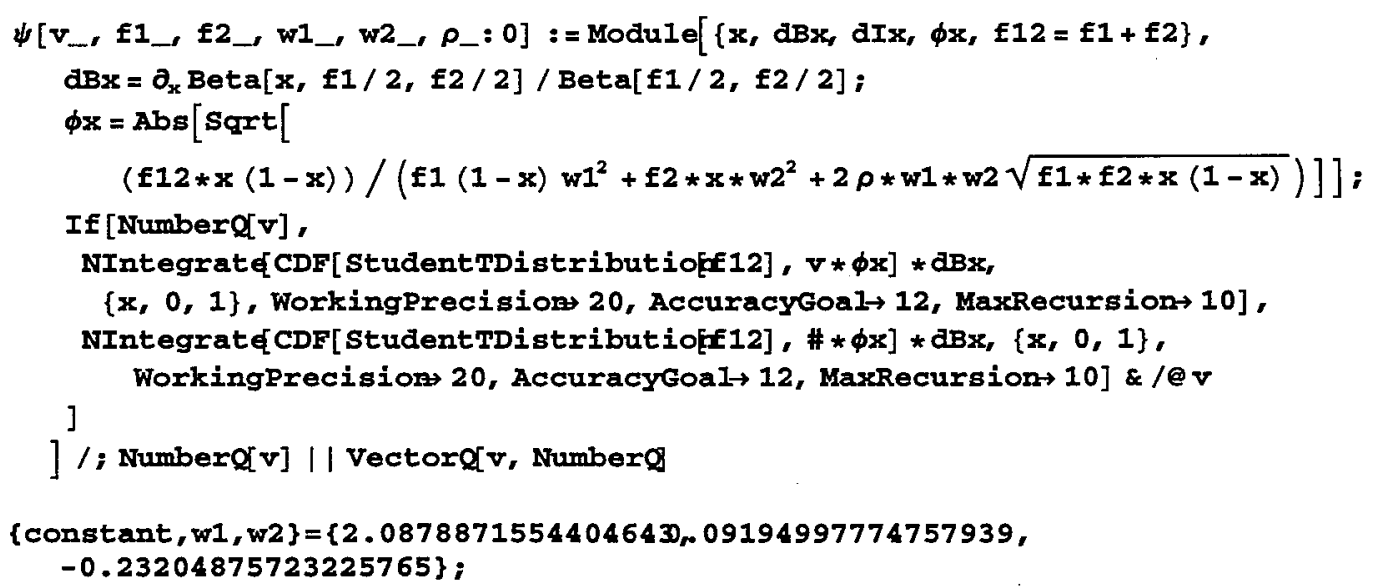




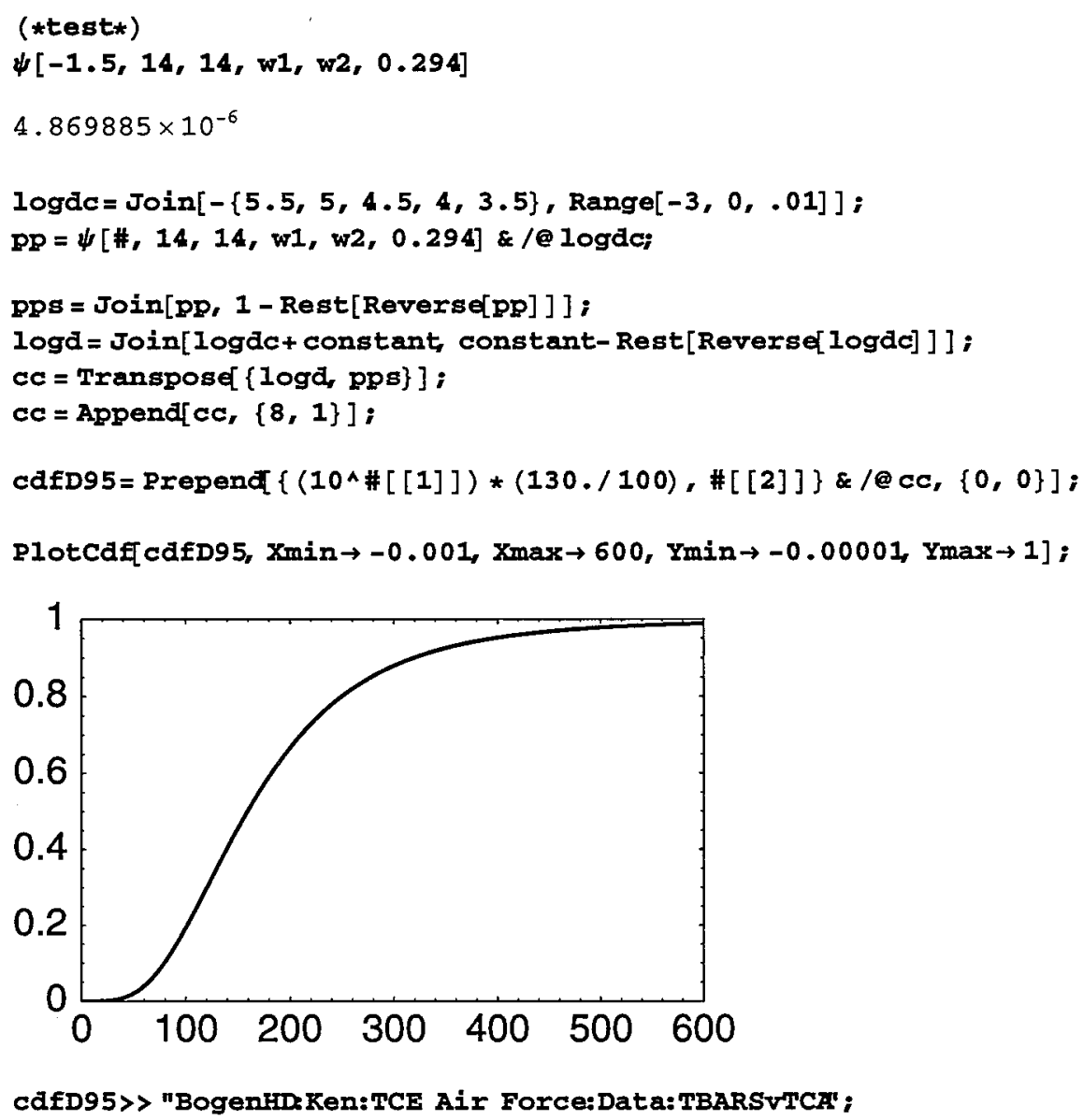

- Obtain a low-risk analytic approximation of cdfD95 that will be used to calculate 2nd order approximations of cytotoxic risk (RcAng, RcBar), because cdfD95 is very nonlinear at low risks

First look at cdfD95 in log-log space:

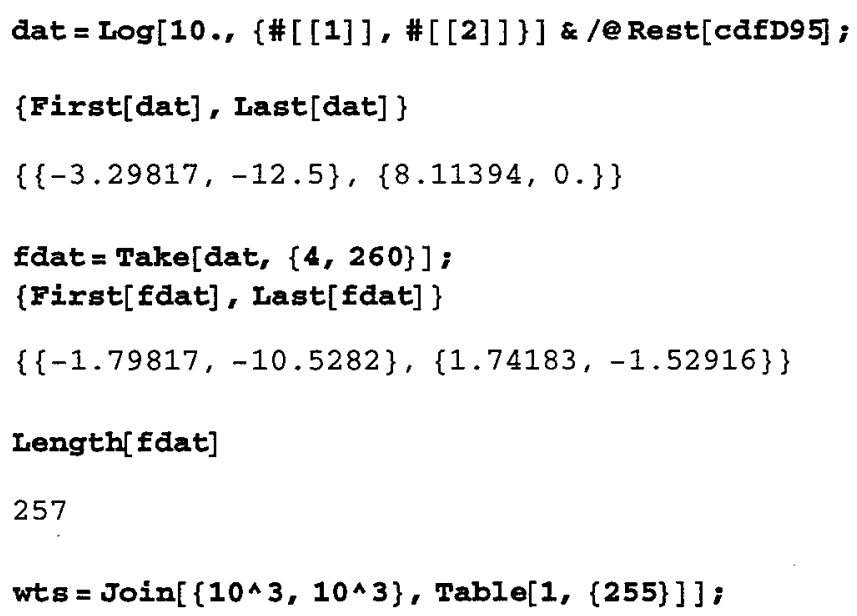


fit $=$ Fit $\left[\right.$ fdat $\left.,\left\{1, x, x^{\wedge} 2\right\}, x\right]$

$-7.23848+2.46626 x+0.469885 x^{2}$

PlotData dat, $x \min \rightarrow-2.01, X \max \rightarrow 3$, JoinPoints $\rightarrow$ True,

$Y \min \rightarrow-10.01, Y \max \rightarrow-.00001$ Style $\rightarrow\{00\}, \operatorname{DotSize} \rightarrow .001$, FitTo $\rightarrow\{$ fit, x $\}$, Dashed $\rightarrow .025]$;

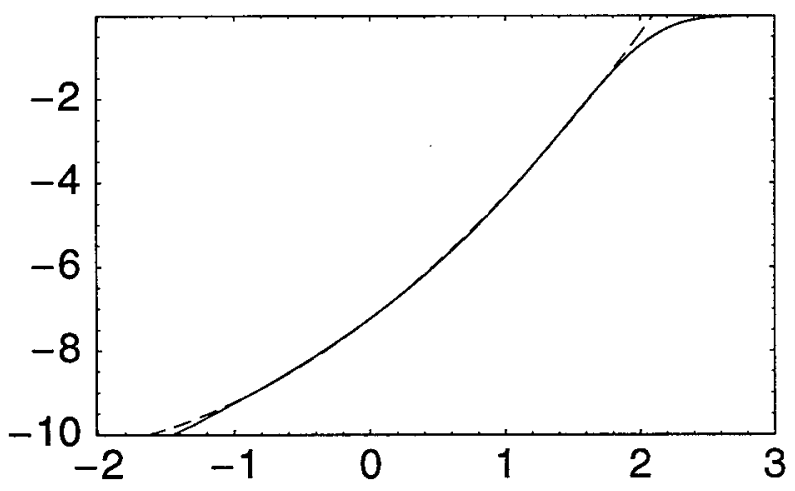

end

end 


\section{Appendix 3.H}

\section{TCE Risk}

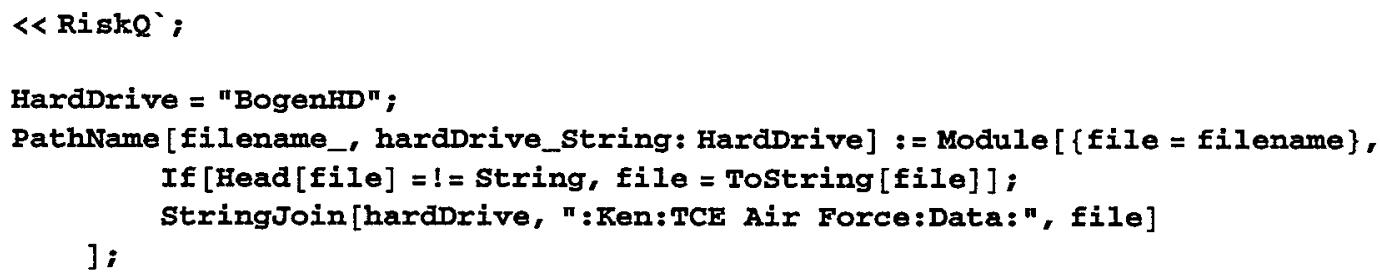

\section{Log-Transform Utility Functions}

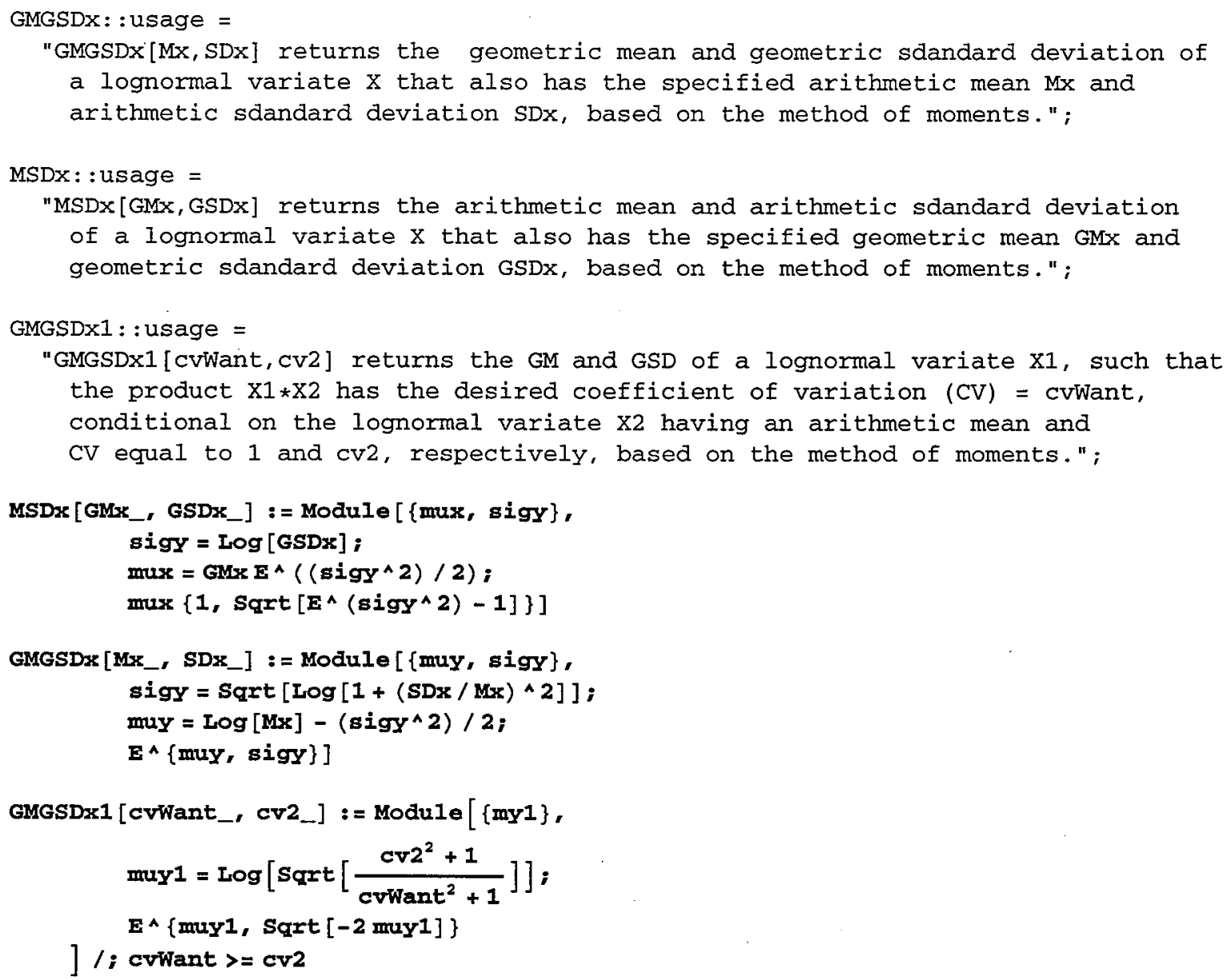




\section{- Extrapolation Factors (cf. Slob \& Pieters, Risk Anal., 1998; EPA)}

Assumed that median is central target for uncertain EF, expected value is central target for heterogeneous EF.

EFinterspTdyn: Uncertain (Median $=1$ )

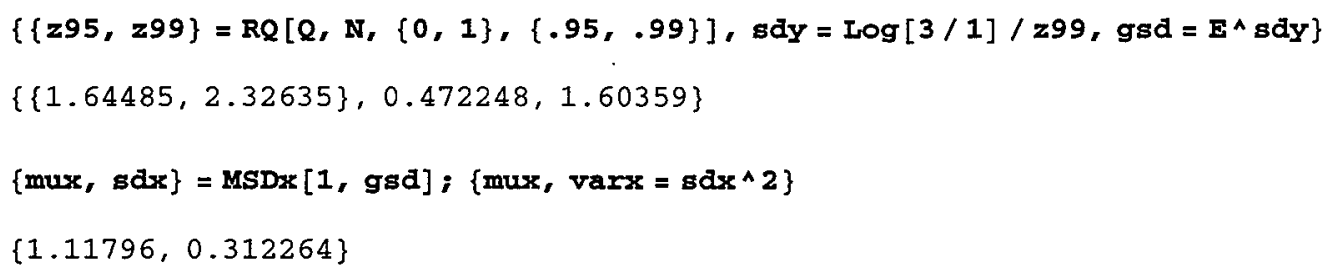

EFintraspTdyn: Heterogeneous (Mean $=1$ )

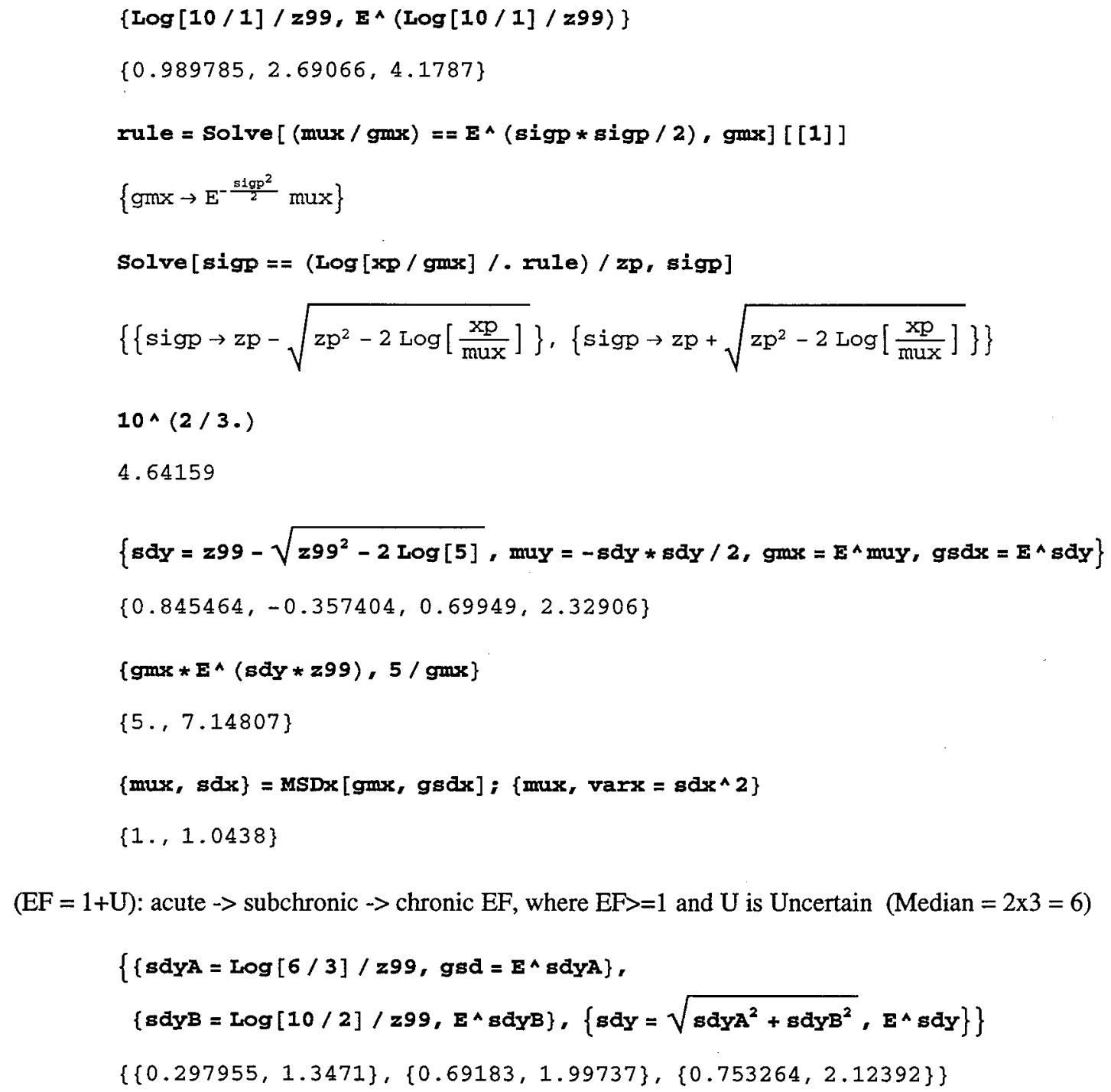




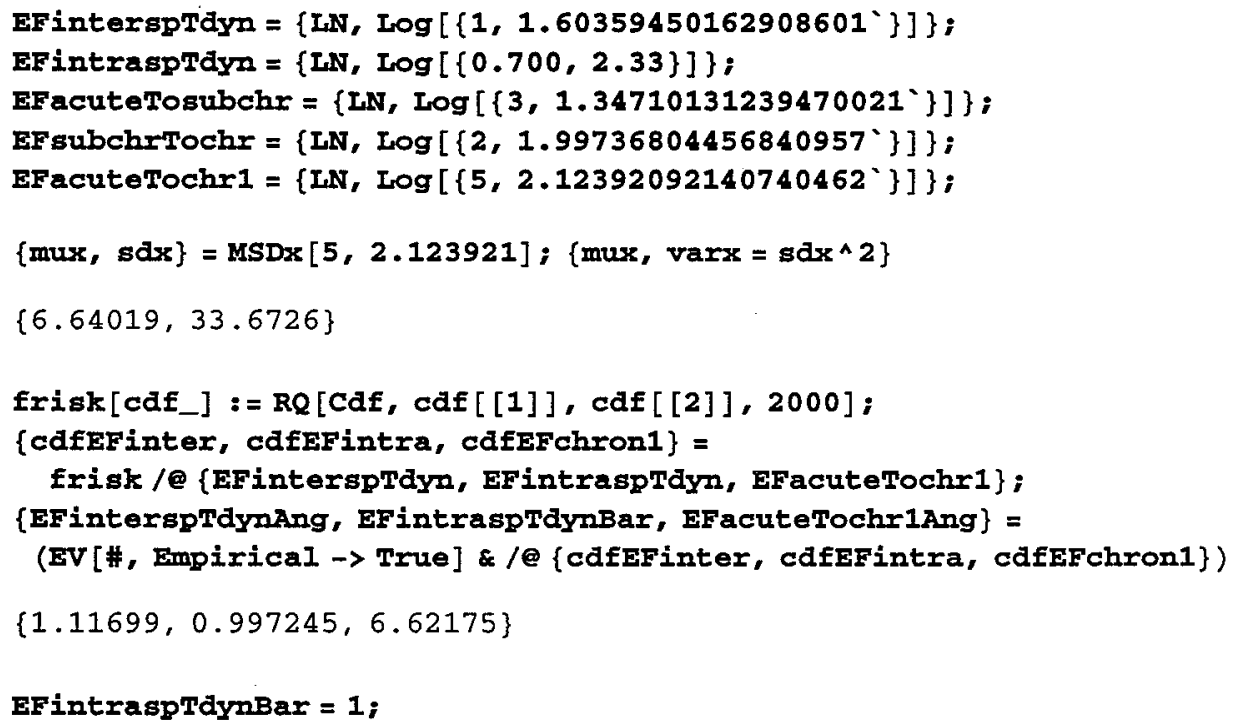

Composite toxicodynamic uncertainty factor $\mathrm{U}=(\operatorname{UinterTdyn} *(1+$ Uacute $2 \mathrm{chr} 1))$, has mean and variance:

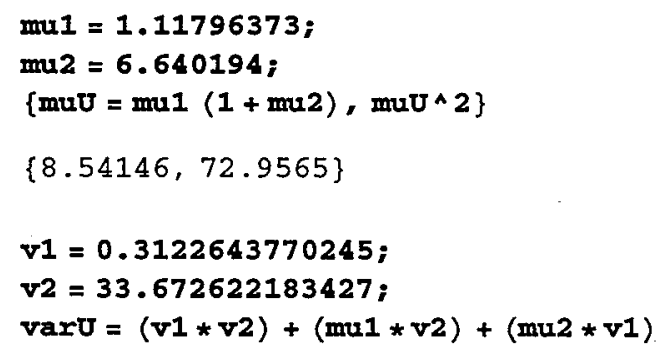

\section{Effective Dose}

\section{- Genotoxic effective dose (EgBar, EgAng)}

Exposures are all in units of (mg/kg-d) x 1000 (see "D. Effective Genotoxic Dose"):

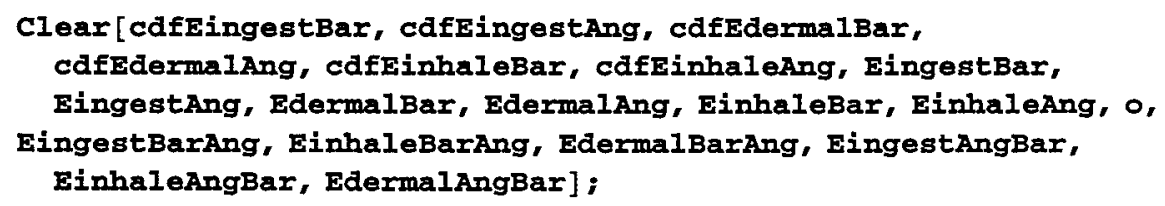


$0=$ Get $/ @($ PathName $[\# 1] \&) / @$

\{EingestBar, EingestAng, EdermalBar, EdermalAng, EinhaleBar, EinhaleAng\};

\{cdfEingestBar, cdfEingestAng, cdfEdermalBar, cdfEdermalAng, cdfEinhaleBar, cdfEinhaleAng $=0$;

\{EingestBarAng, EingestAngBar, EdermalBarAng, EdermalAngBar,

EinhaleBarAng, EinhaleAngBar $\}=($ EV [\#, Empirical $\rightarrow$ True] \& /@ o);

$0=\{\{$ EingestBarAng, EinhaleBarAng, EdermalBarAng $\}$,

\{EingestangBar, EinhaleAngBar, EdermalangBar\}\}

$\{\{0.0507837,0.00235084,0.0071258\},\{0.0491639,0.00225019,0.00684773\}\}$

Plus @ \& / 0

$\{0.0602603,0.0582618\}$

cdfBar $=\left\{\left(\left\{\right.\right.\right.$ EingestBarAng $\left.\left.{ }^{-1}, 1\right\} \#\right) \& / @$ cdfEingestBar,

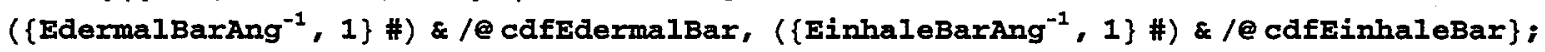
cdfAng $=\left\{\left(\left\{\right.\right.\right.$ EingestAngBar $\left.\left.{ }^{-1}, 1\right\} \#\right) \& / @$ cdfEingestAng ,

( $\left\{\right.$ EdermalAngBar $\left.{ }^{-1}, 1\right\}$ \#) \&/@ cdfEdermalAng, (\{EinhaleAngBar $\left.\left.{ }^{-1}, 1\right\} \#\right) \& / @$ cdfEinhaleAng $\}$;

Plotcdf [Join [cdfBar, cdfAng], Xmin $\left.\rightarrow-10^{-4}, \mathrm{xmax} \rightarrow 5, \operatorname{Ymin} \rightarrow-10^{-4}\right]$;

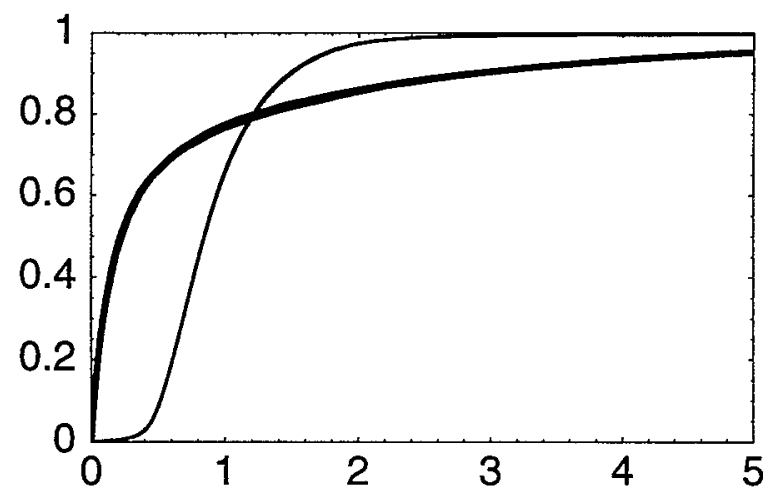

Clear [EgBar, EgAng];

cdfBar = (Plus ee cdfBar) / 3;

cdfAng = (Plus @e cdfAng) $/ 3$;

Put [cdfBar, PathName [EgBar] ];

Put [cdfAng, PathName [EgAng] ];

EgBar = cdfBar;

EgAng = cdfAng;

EgBar $=$ Get $[$ PathName $[$ EgBar $]]$;

EgAng $=$ Get $[$ PathName $[$ EgAng $]] ;$ CdfQ /@ [EgBar, EgAng

\{True, True\} 
PlotCaf [ EgBar, EgAng $\left.\}, X \min \rightarrow-10^{-4}, X_{\max } \rightarrow 5, \operatorname{Ymin} \rightarrow-10^{-4}\right]$;

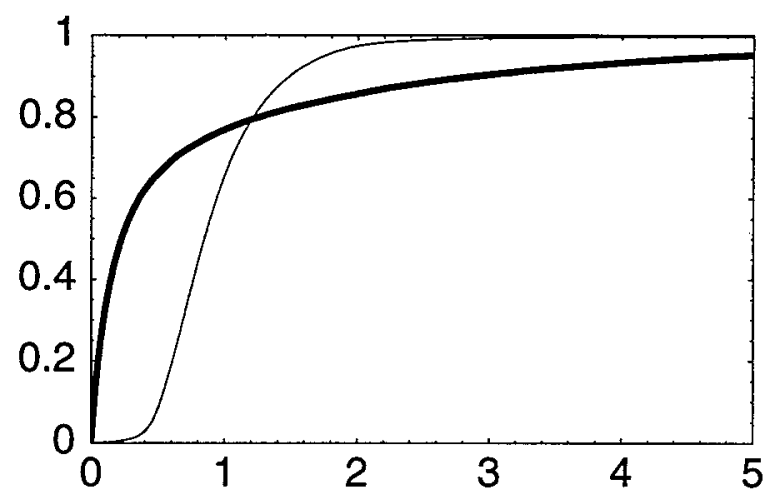

EV [\#, Empirical $\rightarrow$ True] \& /@ [EgBar, EgAng

$\{1,1$.

$\operatorname{Var}[\#$, Empirical $\rightarrow$ True $] \& / @\{$ EgBar, EgAng $\}$

$\{2.45519,4.42109\}$

$\{$ Edf $[\#, 1], \operatorname{Idf}[\#,\{.5, .95, .99\}]\} \& / @\{$ EgBar, EgAng $\}$

$\{\{0.658518,\{0.844739,1.74748,2.59973\}\},\{0.769714,\{0.223819,4.81475,10.326\}\}\}$

\{ $\{$ EingestAngBar, EinhaleAngBar, EdermalAngBar\} /

(Plus e@ \{EingestAngBar, EinhaleAngBar, EdermalAngBar\}), \{EingestBarAng, EinhaleBarAng, EdermalBarAng\} /

(Plus \{EingestBarAng, EinhaleBarAng, EdermalBarAng\}) \}

$\{\{0.843844,0.038622,0.117534\},\{0.842738,0.0390114,0.11825\}\}$

Redefine \{EingestAngBar,EdermalAngBar, EinhaleAngBar\} each as a mean of the corresponding AngBar and BarAng means, then derive relative contributions of \{EingestAngBar,EdermalAngBar, EinhaleAngBar $\}$ to Etotal, (where Etotal = EingestAngBar + EdermalAngBar + EinhaleAngBar).

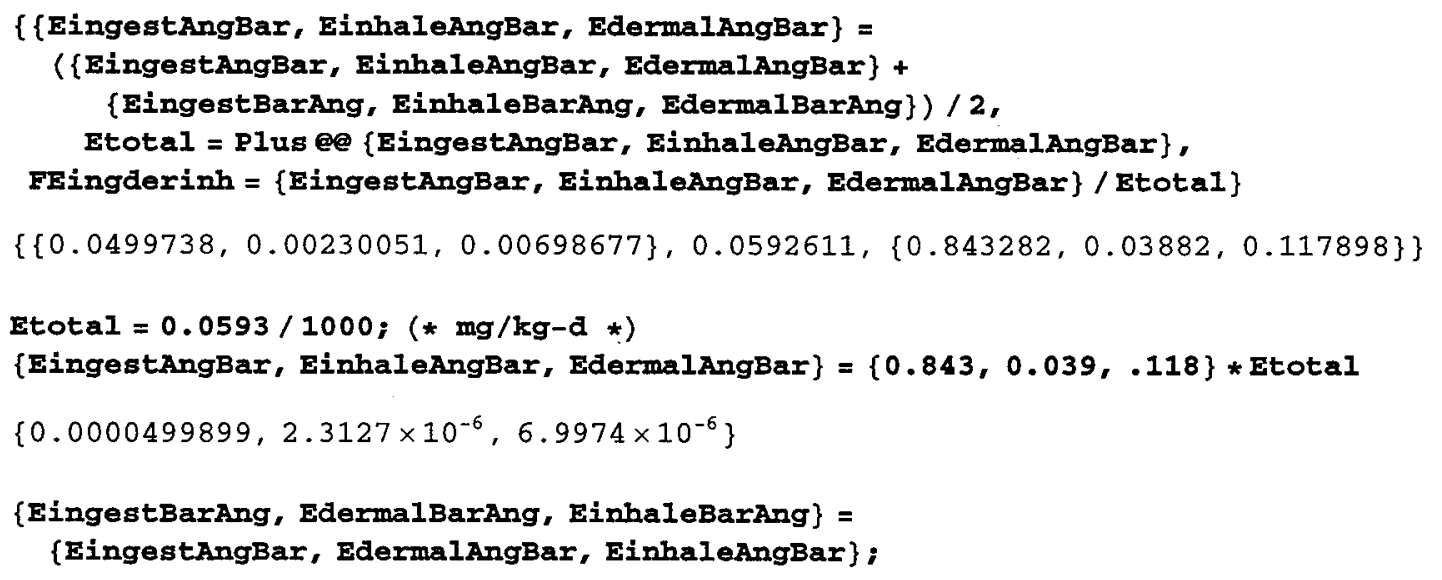

end 


\section{- Cytotoxic exposures (EcBar, EcAng)}

Clear[ECingestAng, ECingestBar, ECinhaleAng, ECinhaleBar, ECdermalAng, ECdermalBar]; ECingestang = Get [PathName [ECingestang] ] ; ECingestBar = Get [PathName [ECingestBar] ] ; ECinhaleAng = Get $[$ PathName [ECinhaleAng $]$; ECinhaleBar = Get [PathName [ECinhaleBar] ] ; ECdermalAng = Get [PathName [ECdermalAng] ] ; ECdermalBar = Get [PathName [ECdermaIBar] ] ; CdfQ /@ \{ECingestAng, ECingestBar, ECinhaleAng, ECinhaleBar, ECdermalAng, ECdermalBar\}

\{True, True, True, True, True, True\}

\{ECingestAngBar, ECingestBarAng, ECinhaleAngBar, ECinhaleBarAng, ECdermalAngBar, ECdermalBarAng $\}=$ EV [\#, Empirical $\rightarrow$ True] \& /@ \{ECingestAng, ECingestBar, ECinhaleAng, ECinhaleBar, ECdermalAng, ECdermalBar\}

$\{0.0161848,0.0162664,0.00835208,0.00840379,0.00226194,0.0022786\}$

cdfCBax $=\left\{\left(\left\{\right.\right.\right.$ ECingestBaxAng $\left.\left.{ }^{-1}, 1\right\} \#\right) \& / @$ ECingestBar,

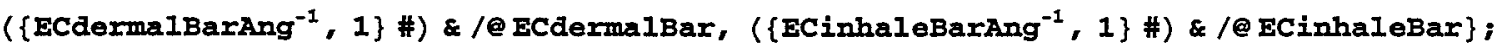
cdfCAng $=\left\{\left(\left\{\right.\right.\right.$ ECingestangBar $\left.\left.{ }^{-1}, 1\right\} \#\right) \& / @$ ECingestang,

( $\left\{\right.$ ECdermalAngBar $\left.{ }^{-1}, 1\right\}$ \#) \& /@ ECdermalAng, (\{ECinhaleAngBar $\left.{ }^{-1}, 1\right\}$ \#) \&/@ ECinhaleAng $\}$;

cdfo /@ \# \& / \{cdfCBar, cdfcAng

\{ True, True, True\}, \{True, True, True $\}$

Plotcdf [Join [cdfCBar, cdfCAng], $x \min \rightarrow-10^{-4}, \mathrm{Xmax} \rightarrow 5, \operatorname{Ymin} \rightarrow-10^{-4}$ ];

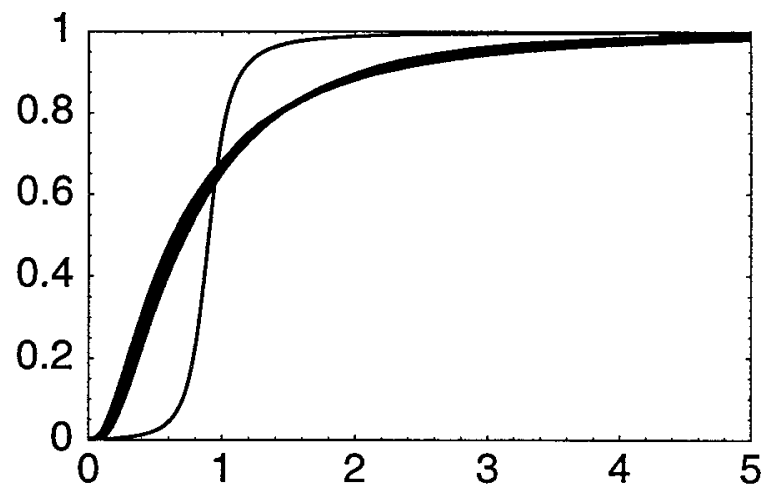

Dimensions / e cdfcAng

$\{\{405,2\},\{405,2\},\{405,2\}\}$

Clear [EcBar, EcAng];

cdfCBar = (Plus eedfCBar) $/ 3$;

cdfCAng $=$ (PIus @edfCAng) $/ 3$;

Put [cdfCBar, PathName [EcBar] ] ;

Put [cdfCAng, PathName [EcAng] ];

EcBar = cdfCBar;

EcAng = cdfCAng; 


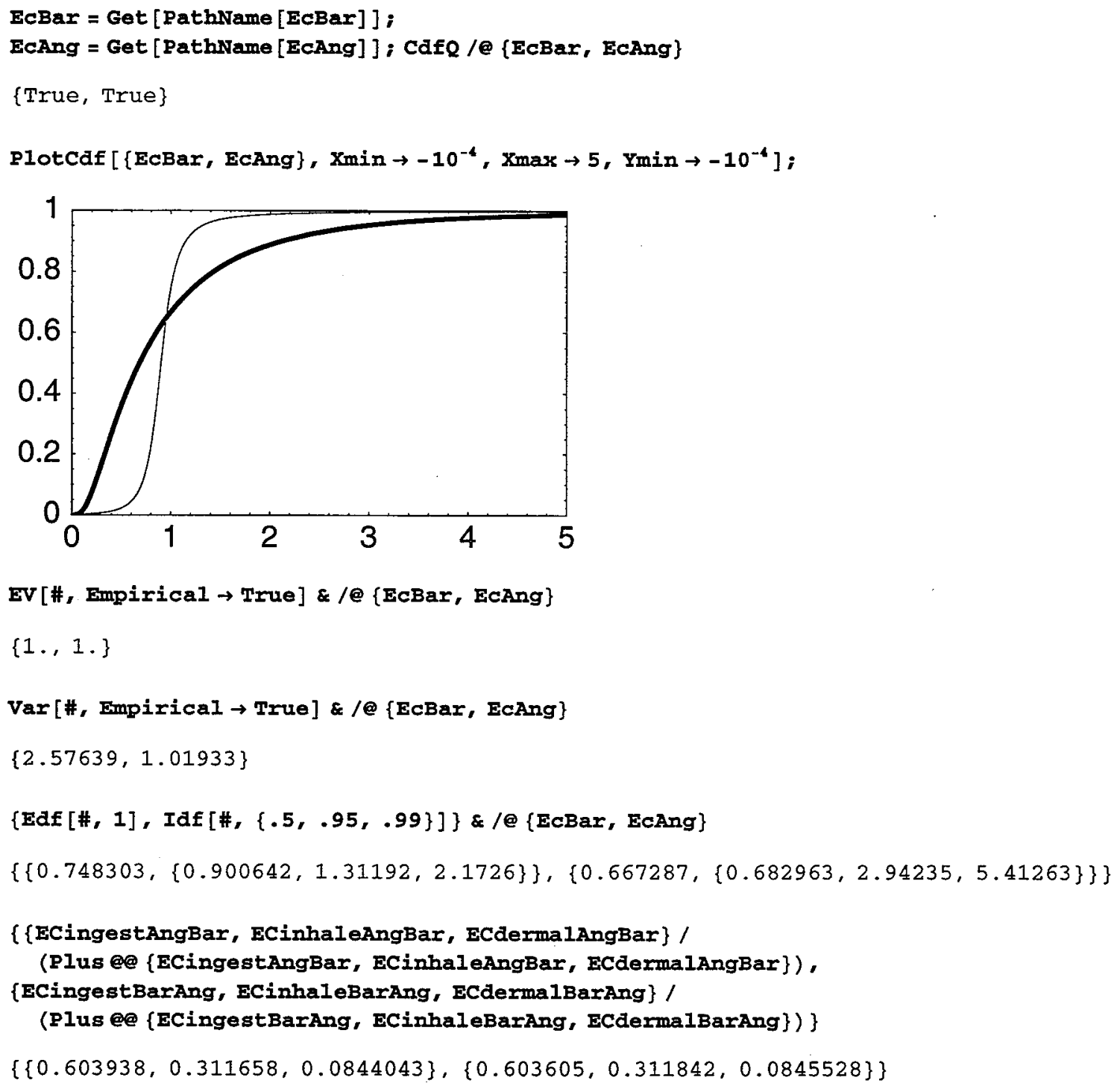

Define \{CingestAngBar,CdermalAngBar, CinhaleAngBar\} each as a mean of the corresponding AngBar and BarAng means, then derive relative contributions of $\{$ CingestAngBar,CdermalAngBar, CinhaleAngBar $\}$ to ECtotal, (where Ctotal = CingestAngBar + CdermalAngBar + CinhaleAngBar).

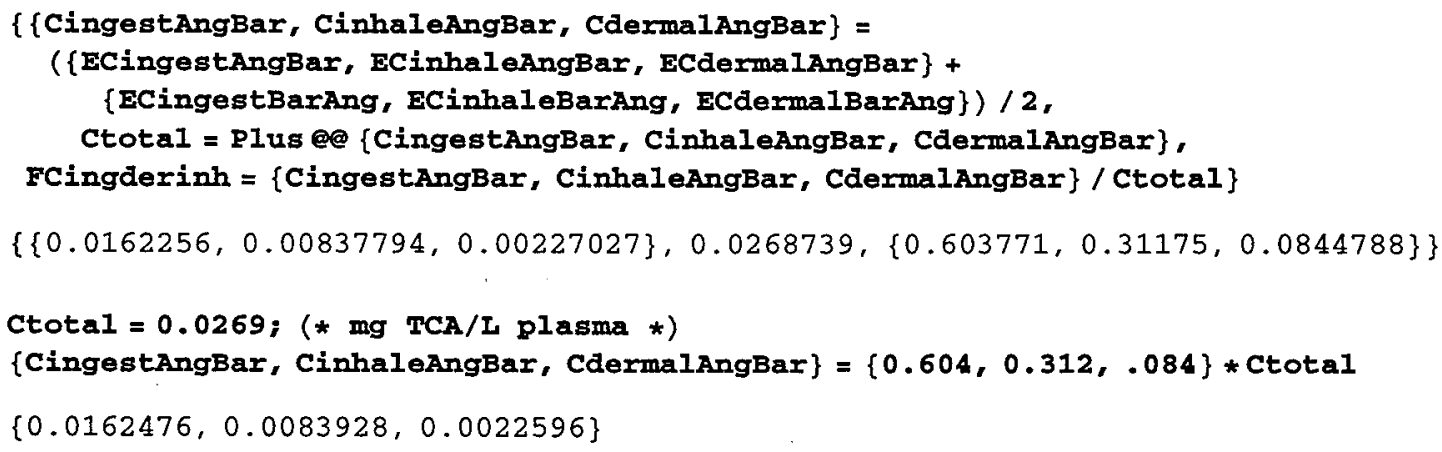


\{CingestBarAng, CdermalBarAng, CinhaleBarAng\} =

\{CingestangBar, CdermalAngBar, CinhaleAngBar\};

end

end

\section{Dose-Response}

- Genotoxic Potency (Qcdf)

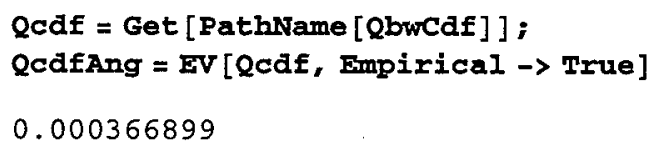

end

\section{- Cytotoxic Potency (TBARSvTCA)}

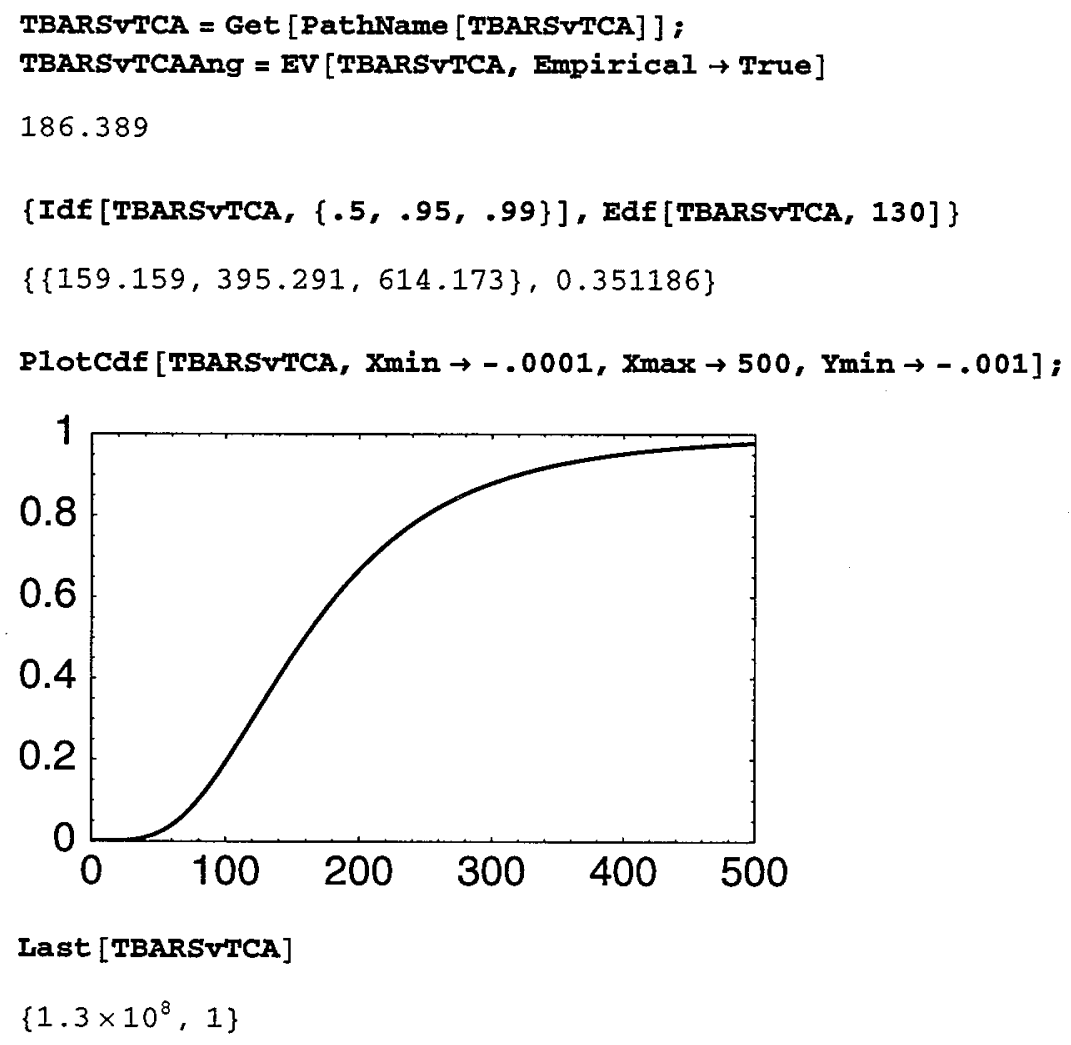

Last [TBARSVTCA]

$\left\{1.3 \times 10^{8}, 1\right\}$

pTBARS $\left[D_{\mathrm{Ca}}\right]=F_{\mathrm{C}}\left(D_{\mathrm{Ca}}\right)$ for effective acute cytotoxic dose $D_{\mathrm{Ca}}$, i.e., the risk of significant TBARS elevation conditional on $D_{\mathrm{Ca}}$. 
pTBARS = Interpolation $\left[A\right.$ ppend $\left[\right.$ TBARSvTCA, $\left.\left\{10^{9}, 1\right\}\right]$, Interpolationorder $\left.\rightarrow 1\right]$; \{PTBARS [130], PTBARS [0.0269]\}

$\left\{0.351186,7.47274 \times 10^{-11}\right\}$

end

end

\section{TCE Risk ( $<$ R>, Rbar)}

\section{2nd-order approximation terms for RBar and RAng}

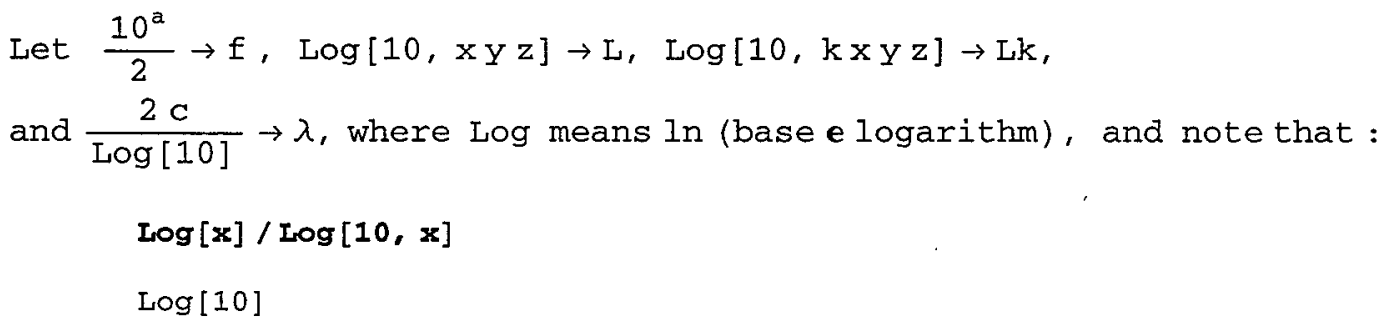

These terms are defined (see Bogen and Spear, 1987) as half the sum of second derivatives with respect to uncertain variates (for RAng), or to heterogeneous variates(for RBar), in the approximate risk function, which is linear-quadratic in Log[10,Dca], where Dca is acute effective cytotoxic dose. Note that, conditional on any variate $\mathrm{x}$ being evaluated at its expected value, the ratio $\left(\sigma^{2}\right)_{x} / x^{2}$ is equal to $\left(\gamma^{2}\right)_{x}$ where $\gamma_{x}$ is the coefficient of variation of $x$ (i.e., $\gamma_{x}=\sigma_{x} / \mu_{x}$ ). By using the latter relationship, simplified expressions derived below can be simplified further by incorporating corresponding coefficients of variation.

\section{- For RBar}

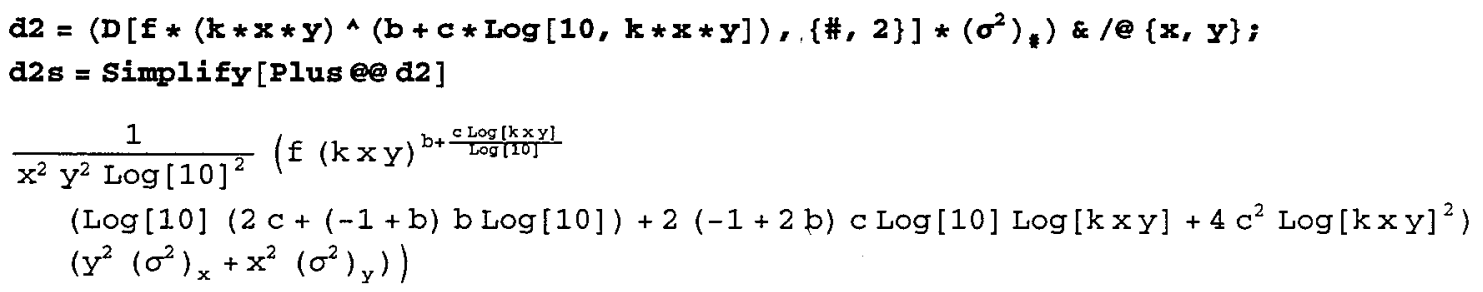

in which $\mathrm{k}=\mathrm{DcaAngBar} * \mathrm{Utdyn}(1+\mathrm{Uch}$ ron)BcBar, $\mathrm{x}=\mathrm{V} 1=\mathrm{Vtdyn}, \mathrm{y}=\mathrm{V} 2=\mathrm{BcAng}$, and the result is evaluated conditional on $\mathrm{V} 1=\mathrm{V} 1 \mathrm{Bar}=\mathrm{VtdynBar}=1$ and on $\mathrm{V} 2=\mathrm{V} 2 \mathrm{Bar}=\mathrm{BAngBar}=1$. (Note that variates Ez are used to denote corresponding normalized dose variates $\mathrm{Bz}$ used elsewhere in this notebook, for all subscripts $\mathrm{z}$.) In simplified form (along with a test that this form is correct):

$$
\text { a2Simplified }=\frac{10^{a}}{2}(k x y)^{b+c L}(\lambda+b(b-1)+2 c L(2(b+c L)-1))\left(\frac{\left(\sigma^{2}\right)_{x}}{x^{2}}+\frac{\left(\sigma^{2}\right)_{Y}}{y^{2}}\right) ;
$$




$$
\begin{aligned}
& \{x 1, x 2\}=N[\{\text { d2s, d2simplified }\}] / / .\{a \rightarrow-2, . b \rightarrow .05, c \rightarrow .15, x \rightarrow .2, y \rightarrow .1, \\
& \left.\quad\left(\sigma^{2}\right)_{x} \rightarrow 1.1,\left(\sigma^{2}\right)_{y} \rightarrow 1.8, L \rightarrow \log [10, k x y], \lambda \rightarrow \frac{2 c}{\log [10]}, k \rightarrow .069, f \rightarrow \frac{10^{\circ}}{2}\right\}
\end{aligned}
$$

$\{20.0338,20.0338\}$

\section{- For RAng}

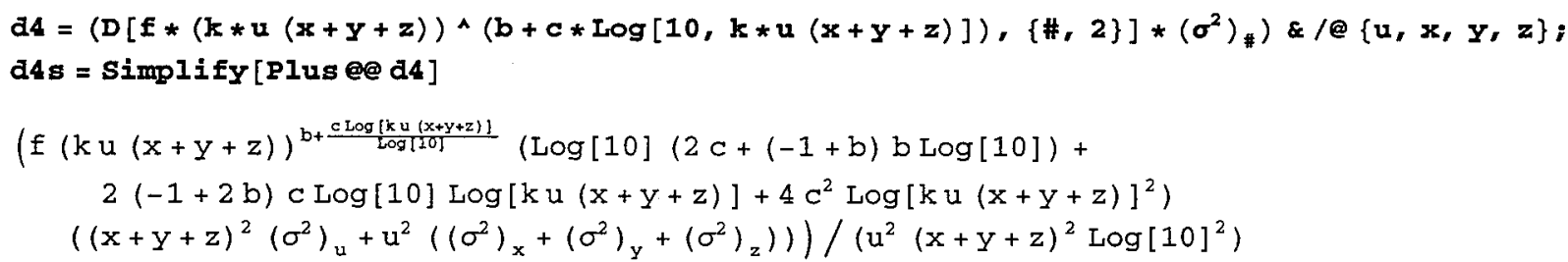

in which $\mathrm{k}=\mathrm{DcaAngBar} * \mathrm{Vtdyn} * \mathrm{BcAng}, \mathrm{u}=\mathrm{U} 1=\mathrm{Utdyn}(1+\mathrm{Uch}$ ron $), \mathrm{x}=\mathrm{U} 2=\mathrm{fCing} * \mathrm{BcBar}, \mathrm{y}=\mathrm{U} 3=\mathrm{fCinh} * \mathrm{BcBar}, \mathrm{z}=$ $\mathrm{U} 4=\mathrm{fCder} * \mathrm{BcBar}$, and the result is evaluated conditional on $\mathrm{U} 1=\mathrm{U} 1 \mathrm{Bar}=[\mathrm{Utdyn}(1+\mathrm{Uchron})] \mathrm{Ang}$ and on $\mathrm{Ui}=\mathrm{UiAng}=$ $\mathrm{fCi} * \mathrm{BcBarAng}=\mathrm{fCi}$ for $\mathrm{i}=\{\mathrm{ing}$, inh, der $\}$. (Note that variates $\mathrm{Ez}$ are used to denote corresponding normalized dose variates $\mathrm{Bz}$ used elsewhere in this notebook, for all subscripts z.) In simplified form (along with a test that this form is correct):

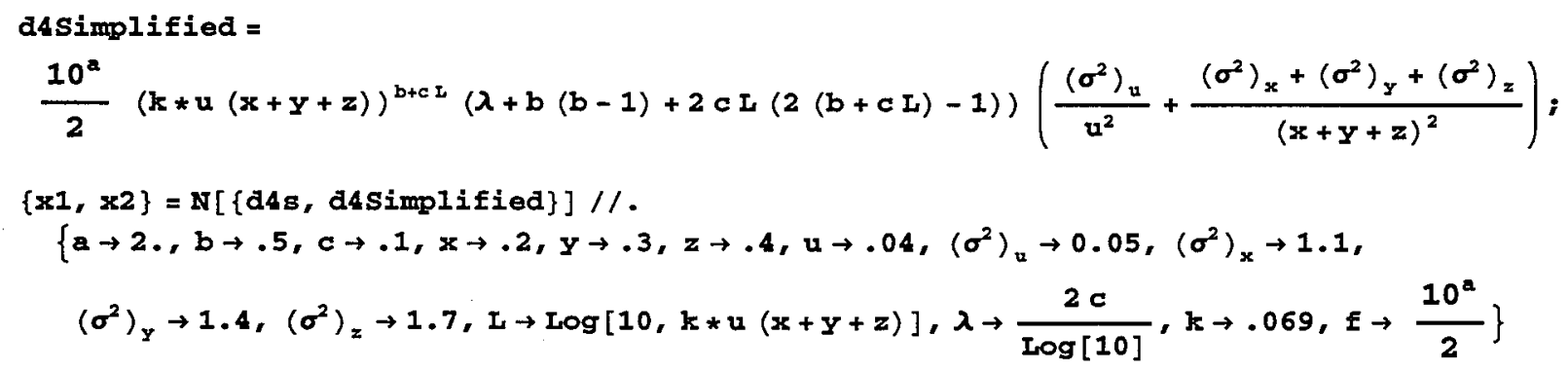

Estimates of variance (e.g., in $\mathrm{Bc}$ ) are used to evaluate the above expressions; e.g., recall that:

$\operatorname{Var}[\#$, Empirical $\rightarrow$ True] \& /Q \{EcBar, EcAng $\}$

$\{2.57639,1.01933\}$

end 


\section{- Rang (Variability Distribution)}

\{EFintraspTdyn, EgAng $\times 3$, EcAng $\times 3\}=7$ heterogeneous variates $\mathrm{Ug}=\mathrm{Likelihood}$ that $\mathrm{Rg}$ is true $\sim \mathrm{U}[0,0.5]$ by assumption; therefore, $\mathrm{UgAng}=1 / 4$. $\{a, b, c\}=$, estimates of linear-quadratic parameters in $\log [10$, Dca $]$ that model $\mathrm{Fc}(\mathrm{Dca})$ (see "Potency.nb")

Dose rank correlations (from "F. Effective Dose Correlations") for: $(\mathbf{n}=50 \times 500)$

\section{GingAng CingAng GinhAng CinhAng GderAng CderAng}

$\begin{array}{llllll}1.0 & 0.233 & 0.878 & -0.00269 & 0.894 & 0.000496 \\ 0.233 & 1.0 .0088 & -0.0088 & 0.421 & 0.00439 & 0.514 \\ 0.878 & -0.0083 & 0.187 & 0.918 & 0.035 \\ -0.00269 & 0.421 & 0.187 & 1.8 & 0.0766 & 0.649 \\ 0.894 & 0.00439 & 0.918 & 0.0766 & 1.0 & 0.177 \\ 0.000496 & 0.514 & 0.035 & 0.649 & 0.177 & 1 .\end{array}$

Assign corresponding values to the upper triangular portion of the simulation-input-variate rank-correlation matrix (which shall be denoted "corr"). Note that the first row of the matrix pertains to the EFintraspTdyn variate (i.e., the intraspecies toxicodynamic extrapolation factor), which is not correlated with any of the 6 exposure variates.

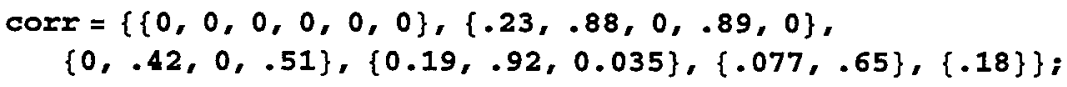

Verify that the Cholesky decomposition of the target rank-corrlation matrix (=Reflect[corr]) contains no imaginary parts:

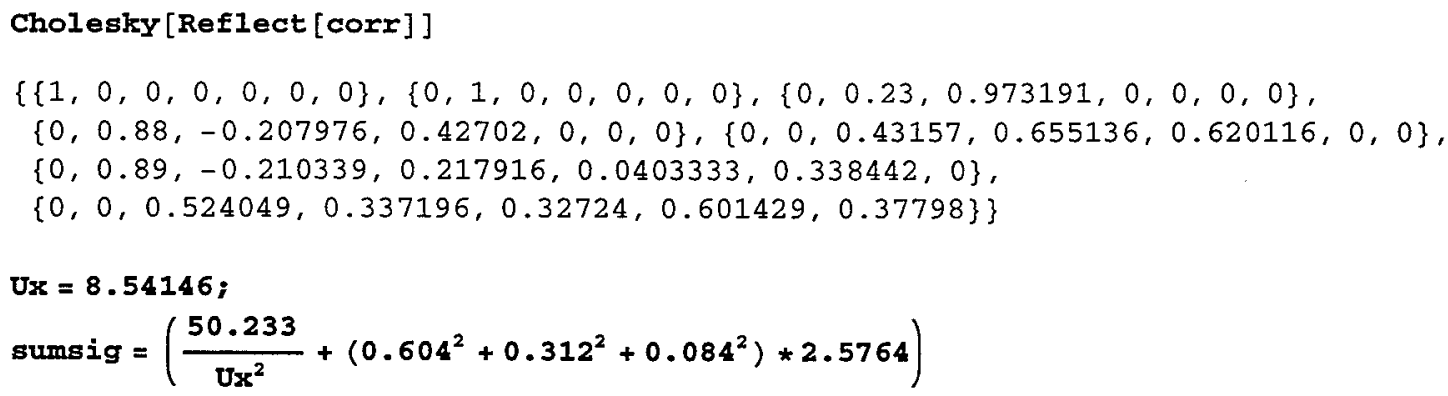




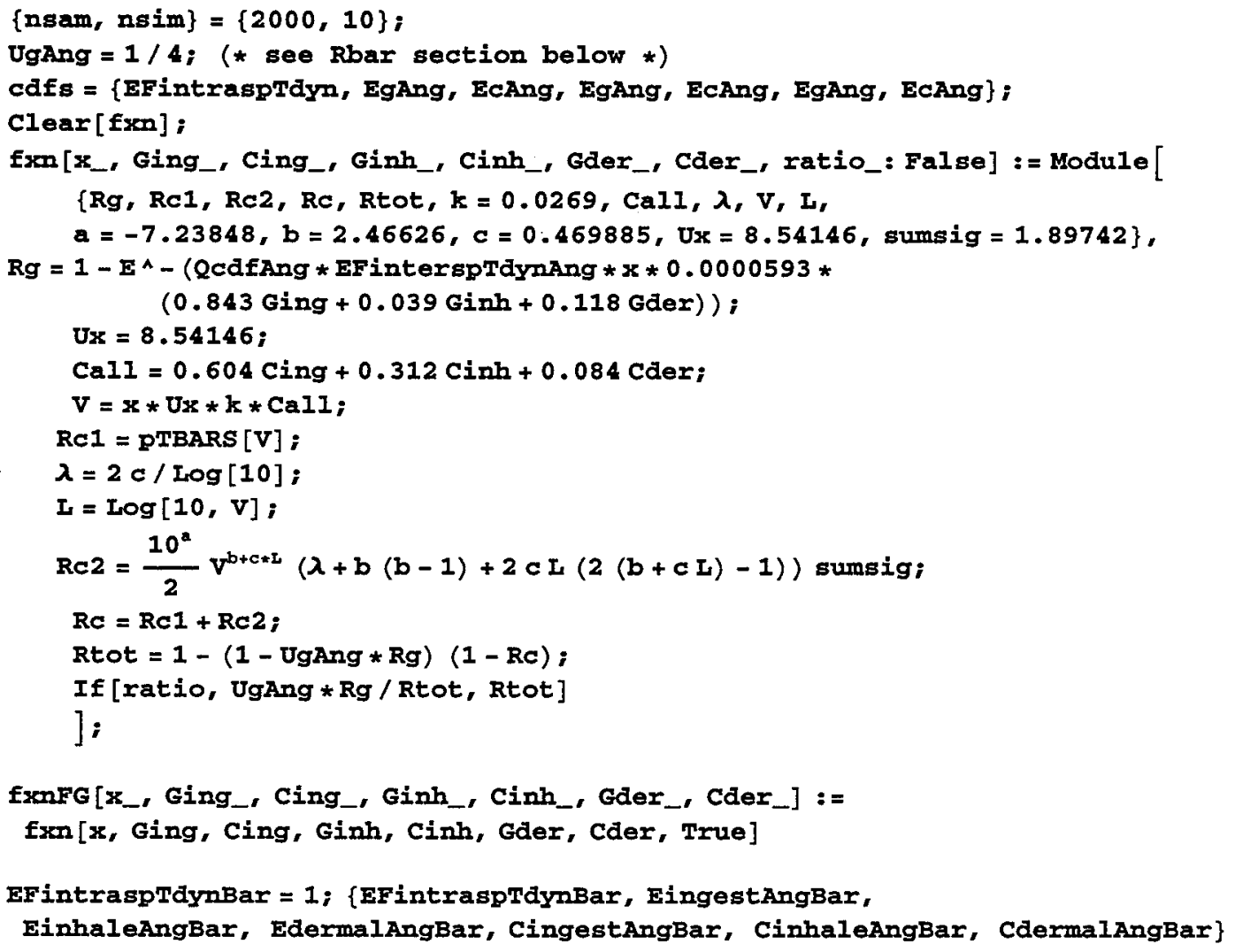

$\left\{1,0.0000499899,2.3127 \times 10^{-6}, 6.9974 \times 10^{-6}, 0.0162476,0.0083928,0.0022596\right\}$

Exn [EFintraspTdynBar, 1, 1, 1, 1, 1, 1]

$1.31708 \times 10^{-8}$

ExnFG [EFintraspTdynBar, 1, 1, 1, 1, 1, 1]

0.461294

Timing $[$ sim1 $=$ Table $[$ Simulatecdf $[$ cdfs, nsam, Correlate $\rightarrow$ corr, Report $\rightarrow$ Append], $\{\mathrm{nsim}\}] ;]$

$\{117.017$ Second, Nul1\}

(* Check Jennrich X2 p-values *)

jenp $=$ Transpose $[$ Range $[$ Length $[$ sim1] $]$, Last /@ Last /e Last /@ sim1 $\}]$

$\{\{1,0.998878\},\{2,0.999285\},\{3,0.971796\},\{4,0.99744\},\{5,0.986883\}$,

$\{6,0.99969\},\{7,0.998695\},\{8,0.986122\},\{9,0.998931\},\{10,0.827001\}\}$

$\{j e n, c d f, c v i n\}=$

QUAnalyze [cdfs, fxn, nsam, nsim, SimIn $\rightarrow$ siml, correlate $\rightarrow$ corr, Scale $\left.\rightarrow 10^{6}\right]$; 
TBL /e jen

\begin{tabular}{|c|c|c|c|c|c|c|c|}
\hline$\left\{\begin{array}{l}\text { Mean }[\Delta r] \\
0.000176653\end{array}\right.$ & $\begin{array}{l}\operatorname{Max}[|\Delta r|] \\
0.0187711\end{array}$ & $\begin{array}{l}\text { Jennrichchi2 } \\
14.9189\end{array}$ & $\begin{array}{l}\text { DegFr } \\
21\end{array}$ & $\begin{array}{l}\text { P-adj } \\
0.99969 .\end{array}$ & $\begin{array}{l}\text { Fractile } \\
0.01 \\
0.05 \\
0.5 \\
0.95 \\
0.99 \\
\text { Mean } \\
\text { Variance }\end{array}$ & $\begin{array}{l}\text { Value } \\
0.0000790371 \\
0.000231529 \\
0.00460686 \\
0.172878 \\
1.36419 \\
0.230061 \\
138.397\end{array}$ & $\begin{array}{l}\text { CVM }(8) \\
2.52229 \\
2.6391 \\
1.47592 \\
2.78787 \\
8.07152 \\
43.1698 \\
94.2987\end{array}$ \\
\hline
\end{tabular}

Plotcdf $[$ cvm, cdf $\}, \operatorname{Ymin} \rightarrow-.01, \mathrm{xmin} \rightarrow-.0001, \mathrm{xmax} \rightarrow 1]$;

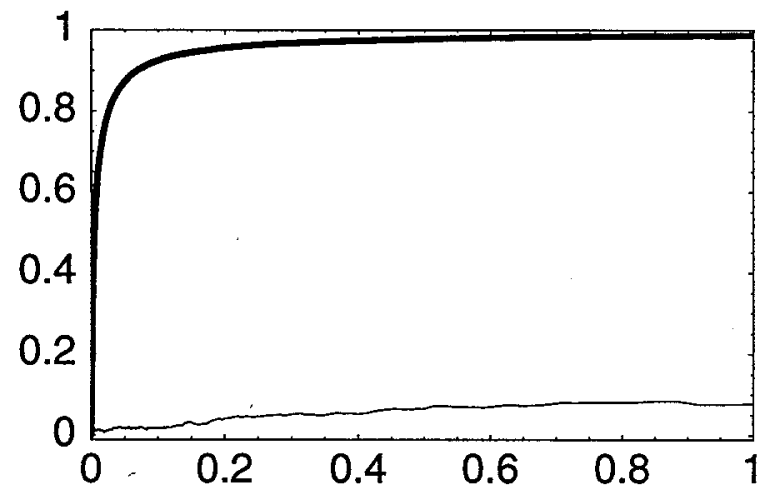

Put [cdf, PathName [Rang] ] ; Rang = cdf;

Rang $=$ Get $[$ PathName $[$ Rang $]] ;$ CdfQ $[$ Rang $]$

True

$\{$ RangBar $=\operatorname{EV}[$ Rang, Empirical $\rightarrow$ True $]$,
$\{$ Rang50, Rang95, Rang99 $\}=\operatorname{Idf}[$ Rang, $\{.5, .95, .99\}]$, Edf $[\operatorname{Rang}, 1]\}$

$\{0.230061,\{0.00460686,0.172878,1.36419\}, 0.987149\}$

Fraction of total risk due to genotoxic risk:

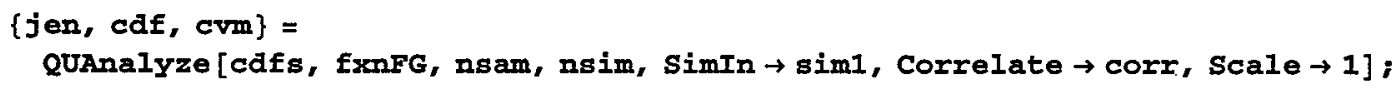

TBL /@ jen

\begin{tabular}{|c|c|c|c|c|c|c|c|}
\hline$\left\{\begin{array}{l}\text { Mean }[\Delta r] \\
0.000466053\end{array}\right.$ & $\begin{array}{l}\operatorname{Max}[|\Delta r|] \\
0.0212522\end{array}$ & $\begin{array}{l}\text { Jennrichchi2 } \\
16.3988\end{array}$ & $\begin{array}{l}\text { DegFr } \\
21\end{array}$ & $\begin{array}{l}\mathrm{P}-\mathrm{adj} \\
\mathrm{I}\end{array}$ & $\begin{array}{l}\text { Fractile } \\
0.01 \\
0.05 \\
0.5 \\
0.95 \\
0.99 \\
\text { Mean } \\
\text { Variance }\end{array}$ & $\begin{array}{l}\text { Value } \\
0.00262033 \\
0.0182746 \\
0.465644 \\
0.967092 \\
0.99007 \\
0.479793 \\
0.107487\end{array}$ & $\begin{array}{l}\operatorname{CVM}(8) \\
5.59528 \\
1.61415 \\
0.531193 \\
0.0530831 \\
0.0407695 \\
0.127858 \\
0.320455\end{array}$ \\
\hline
\end{tabular}

end 


\section{- Rbar (Uncertainty Distribution)}

\{Ug, EgBar, EcBar, Qcdf, EFinterspTdyn, EFacuteTochr1 $\}=6$ uncertain variates $\mathrm{Ug}=$ Likelihood that $\mathrm{Rg}$ is true $\sim \mathrm{U}[0,0.5]$ by assumption

$\{a, b, c\}=$, estimates of linear-quadratic parameters in $\log [10$, Dca $]$ that model Fc(Dca) (see "Potency.nb")

$$
\begin{aligned}
& \text { corx }=\{\{0,0,0,0,0\},\{.49,0,0,0\},\{0,0,0\},\{0,0\},\{0\}\} \\
& \text { sumsig }=\left(\frac{1.0438}{1^{2}}+\frac{1.01933}{1^{2}}\right)
\end{aligned}
$$

2.06313

\{nsam, nsim $\}=\{2000,10\}$;

$\mathrm{Ug}=\{\{0,0\},\{.5,1\}\}$;

cdfs $=\{$ Ug, EgBar, EcBar, Qcdf, EFinterspTdyn, EFacuteTochr1 $\}$;

Clear [fxn];

fxn[ug_, Gal1_, Cal1_, q, uf1_, uf2, ratio_: False] := Module [

\{Rg, Rc1, Rc2, Rc, Rtot, $\mathrm{k}=0.0269$,

$\lambda, U, L, a=-7.23848, b=2.46626, c=0.469885$, sumsig $=2.06313\}$,

$\mathrm{Rg}=1-\mathrm{E}^{\wedge}-(\mathrm{g} * \mathrm{ufl} * 1 * 0.0000593 \mathrm{Gall})$;

$\mathrm{U}=\mathrm{k} * \operatorname{uf1} 1$ * $1+\mathrm{uf2}) * \operatorname{Cal1}$;

$\operatorname{RC1}=$ PTBARS $[\mathrm{U}]$;

$\lambda=2 c / \log [10]$;

$\mathrm{L}=\log [10, \mathrm{U}]$;

$\operatorname{Rc} 2=\frac{10^{a}}{2} U^{b+c * L}(\lambda+b(b-1)+2 c L(2(b+c I)-1)) *$ sumsig;

$\mathrm{RC}=\mathrm{RC1}+\mathrm{RC2}$;

Rtot $=1-(1-u g * R g)(1-R c)$;

If [ratio, ug* Rg/Rtot, Rtot]

;

fxenfG[ug_, Gal1_, Cal1_, qu, uf1_, uf2_] := fxn[ug, Ga11, Cal1, q, uf1, uf2, True]

[QcdfAng, EFinterspTdynAng, EFacuteTochr1Ang\}

$\{0.000366899,1.11699,6.62175\}$

Exn [0.25, 1, 1, QcdfAng, EFinterspTdynAng, EFacuteTochr1Ang]

$1.3516 \times 10^{-8}$

fxnFG[0.25, 1, 1, Qcdfang, EFinterspTdynAng, EFacuteTochr1Ang]

0.449514

Timing $\left[\{j e n, c d f, c v m\}=\right.$ QUAnalyze $\left[\right.$ cdfs, fxn, nsam, nsim, Correlate $\left.\left.\rightarrow \operatorname{corr}, \operatorname{scale} \rightarrow 10^{6}\right] ;\right]$

$\{79.5833$ second, Nu11\} 
TBL / jen

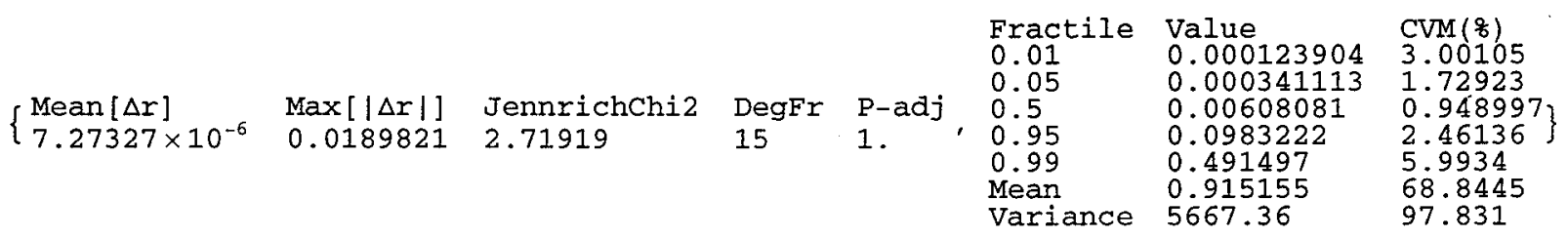

PlotCdf $[\{\mathrm{crm}, \mathrm{cdf}\}, Y \min \rightarrow-.0001, \mathrm{Xmin} \rightarrow-.0001, \mathrm{Xmax} \rightarrow .5]$;

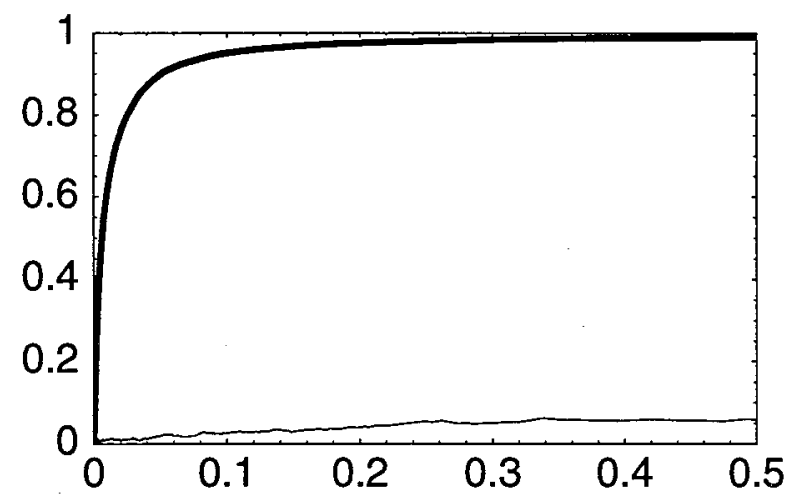

Clear[Rbar];

Put [cdf, PathName [Rbar] ]; Rbar = cdf;

Rbar $=$ Get $[$ PathName $[$ Rbar $]] ;$ CdfQ $[$ Rbar $]$

True

$\{$ RbarAng $=\mathrm{EV}[$ Rbar, Empirical $\rightarrow$ True $]$,

$\{$ Rbar50, Rbar95, Rbar99 $\}=\operatorname{Idf}[\operatorname{Rbar},\{.5, .95, .99\}]$, Edf $[R$ bar, 1] $\}$

$\{0.915155,\{0.00608081,0.0983222,0.491497\}, 0.994501\}$

end

PIotCdf $[\{$ Rang, Rbar $\}, \operatorname{Ymin} \rightarrow-.0001, \mathrm{Xmin} \rightarrow-.000001, \mathrm{Xmax} \rightarrow .01]$;

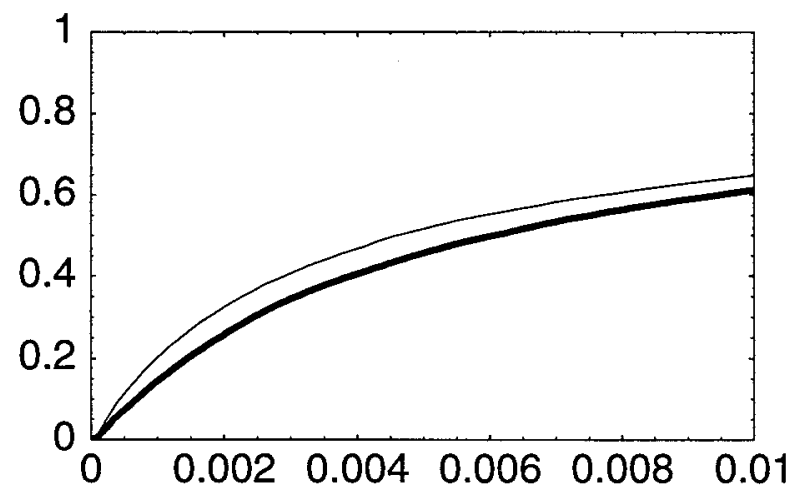


PlotCdf [ Rang, Rbar\}, Ymin $\rightarrow .7999, \mathrm{Xmin} \rightarrow-.000001, \mathrm{Xmax} \rightarrow .5]$;

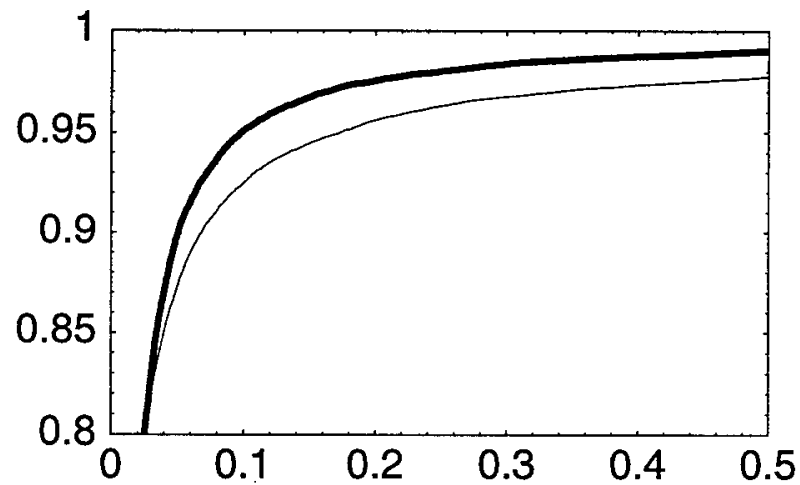

PlotCdf [ Rang, Rbar $\}, Y \min \rightarrow .9899, \mathrm{Xmin} \rightarrow-.000001, \mathrm{Xmax} \rightarrow 15]$;

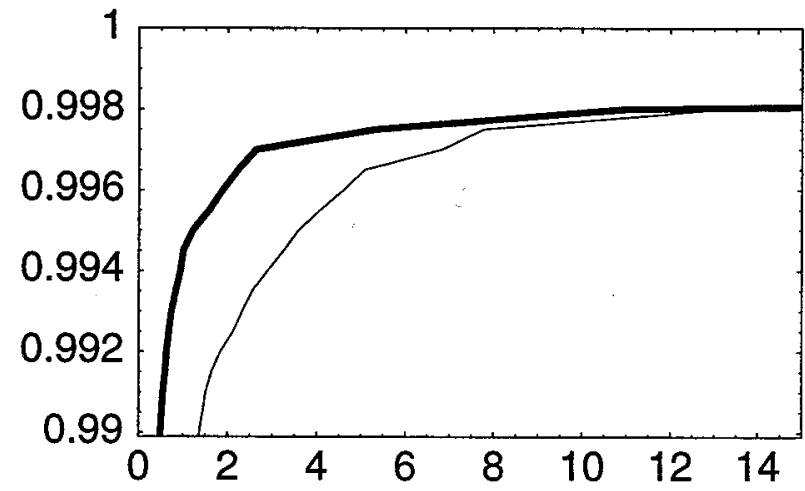

Fraction of total risk due to genotoxic risk:

\{jen, cdf, cvm $\}=$ QUAnalyze $[$ cdfs, fxnFG, nsam, nsim, Correlate $\rightarrow$ corr, scale $\rightarrow 1]$;

TBL /@ jen

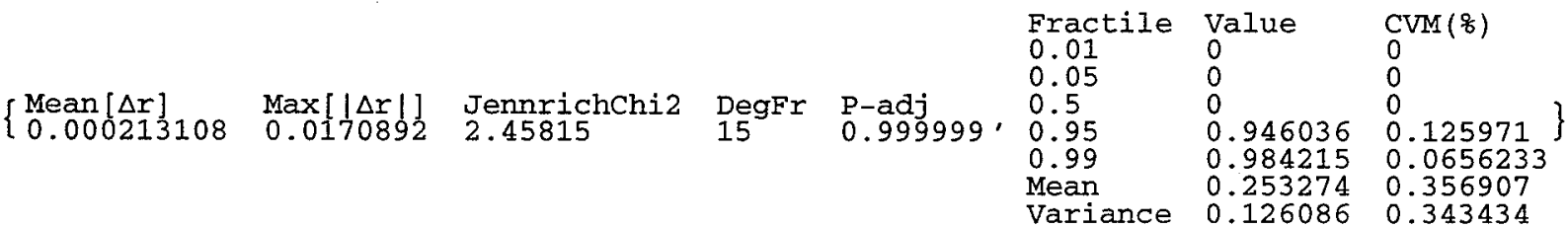

$\operatorname{Edf}[\mathrm{cdf}, 0]$

0.553223

end 


\section{Confidence Bounds on JUV in Risk}

- $\mathbf{R}^{* 99}$ = Analytic upper-bound JUV estimator (@ 99th \%ile on U \& V)

\{Rang99, RangBar, Rbar99, RbarAng\}

$\{1.36419,0.230061,0.491497,0.915155\}$

$\mathrm{R} * 99 \mathrm{~b}=$ rho99 $\times$ Rang99, rho99 $=(\operatorname{Rbar} 99) /(<\mathrm{Rbar}>) \quad<----$ As defined by Bogen $(1995)$

$\mathrm{R}^{* 99 \mathrm{a}}=$ rho99 $\times \mathrm{Rbar} 99$, tho99 $=(<\mathrm{R}>99) /(<\mathrm{R}>$ bar $) \quad<----$ Alternative definition

(* CV for R99* *)

\{RbarAngCv, Rbar99Cv, RangBarcv, Rang99Cv $\}=\{.688, .0599, .432, .0807\}$;

$\{$ rhoa $=$ (Rang99 $/$ RangBar $)$, rhob $=($ Rbar99 $/$ RbarAng $)\}$

$\{5.92966,0.537064\}$

$\left(\left(1-\left(1-\#[[1]]^{2}\right)\left(1-\#[[2]]^{2}\right)\right)^{.5}\right) \& / @\{$

\{Rang99Cv, RangBarcv\}, \{Rbar99cv, RbarAngcv\}\}

$\{0.438088,0.689372\}$

$\{R 99 a=R b a r 99 *$ rhoa, R99b $=$ Rang99 * rhob, R99a / R99b $\}$

$\{2.91441,0.732656,3.97787\}$

$\{$ gammaa, gammab $\}=\left(\left(\left(1-\left(1-\#[[1]]^{2}\right)\left(1-\#[[2]]^{2}\right)\left(1-\#[[3]]^{2}\right)\right)^{.5}\right) \& / @\{\right.$

\{Rbar99cv, Rang99cv, RangBarcv\}, \{Rang99cv, Rbar99cv, RbarAngcv\}\})

$\{0.441385,0.691846\}$

- R*99 = Target-Nested Monte-Carlo JUV estimators (@ 99th \%ile on V)

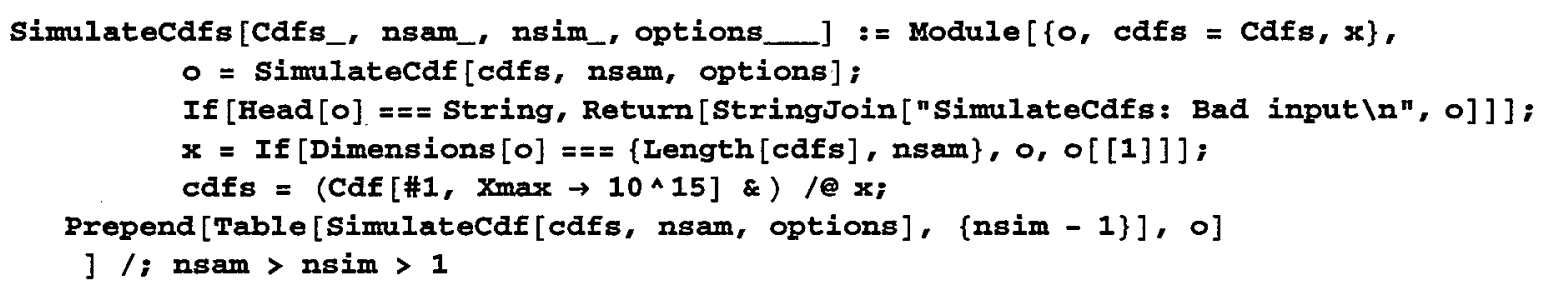




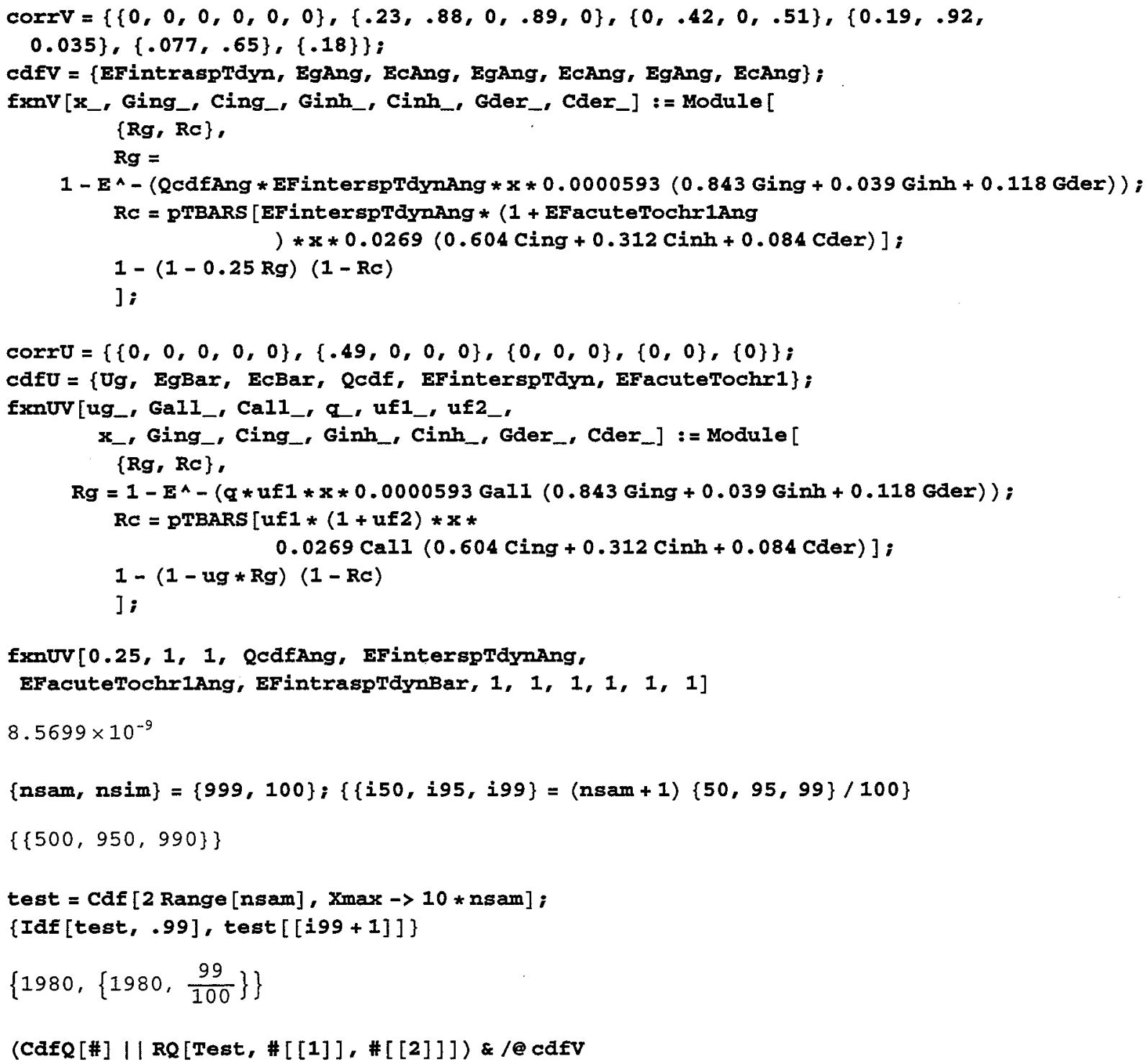


Timing $[$ jen, cdf, cvm $\}=$ QUAnalyze $\left[13\right.$, fxnUV, nsam, nsim, $\left.\left.\operatorname{simIn} \rightarrow \operatorname{simin}, \operatorname{Scale} \rightarrow 10^{6}\right] ;\right]$

$\{35.0667$ Second, Nul1\}

$\min (499.067+35.067) / 60$

$8.90223 \mathrm{~min}$

TBI [jen]

Fractile Value CVM $(q)$

$\begin{array}{lll}0.01 & 0.00191737 & 4.40108 \\ 0.05 & 0.00557622 & 4.31837\end{array}$

$0.5 \quad 0.0990887 \quad 4.15774$

$0.95 \quad 4.08454 \quad 4.93046$

$0.99 \quad 32.9278 \quad 5.67837$

Mean $121.391 \quad 17.1954$

Variance $5.42842 \times 10^{7} \quad 27.8288$

Plotcdf $[\{$ cvm, cdf $\}, Y \min \rightarrow-.01, x \min \rightarrow-.0001, \mathrm{Xmax} \rightarrow 5]$;

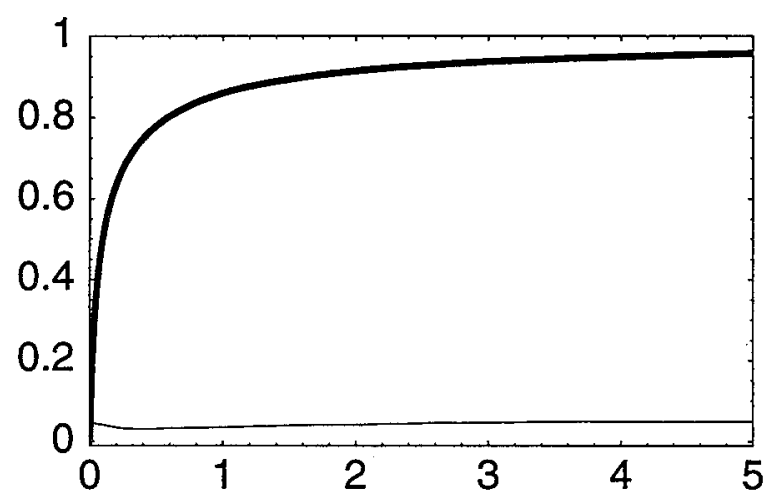

end

\section{- R*99 = Traditional Nested Monte-Carlo JUV estimators (@ 99th \%ile on V)}

Compare variability fractiles for the 50th, 95th and 99th \%ile with respect to uncertainty, respectively, obtained using a traditional nested Monte-Carlo approach:

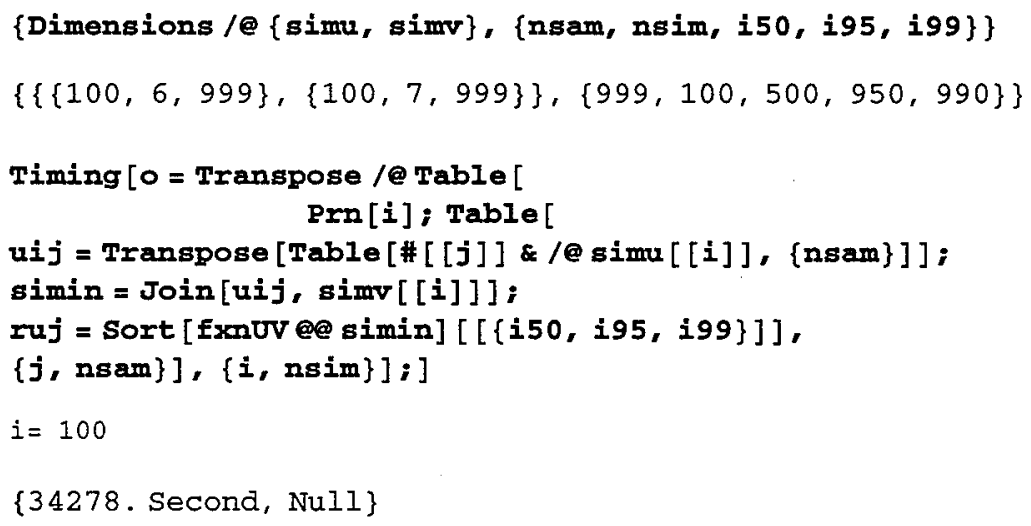


$\{38278 .(60 . \wedge-2) h, 38278 . /(499.067+35.067)\}$

$\{10.6328 \mathrm{~h}, 71.6637\}$

\{Dimensions [0], nsam, nsim\}

$\{\{100,3,999\}, 999,100\}$

$\mathbf{f x}\left[r_{-}\right]:=r$

in = List /@ \#\&/@ Transpose [0] ;

Dimensions [in]

$\{3,100,1,999\}$

$\{j e n$, caf, cvm $\}=$ Transpose $\left[\right.$ QUAnalyze $\left[1, \mathrm{fx}\right.$, nsam, nsim, $\operatorname{simIn} \rightarrow \#$, Scale $\left.\left.\rightarrow 10^{6}\right] \& / @ i n\right] ;$

Variability fractiles for the 50th, 95th and 99th \%ile with respect to uncertainty, respectively:

TBL /@ jen

\begin{tabular}{|c|c|c|c|c|c|}
\hline $\begin{array}{l}\text { Fractile } \\
0.01 \\
0.05 \\
0.5 \\
0.95 \\
0.99 \\
\text { Mean } \\
\text { Variance }\end{array}$ & $\begin{array}{l}\text { Value } \\
0.0000280892 \\
0.0000751209 \\
0.000966502 \\
0.0117496 \\
0.033135 \\
0.0292517 \\
10.4468\end{array}$ & $\begin{array}{l}\text { CVM }(8) \\
1.40513 \\
0.711428 \\
0.385091 \\
0.721707 \\
1.59806 \\
34.0115 \\
69.2366\end{array}$ & $\frac{8}{7}$ & & \\
\hline $\begin{array}{l}\text { Fractile } \\
0.01 \\
0.05 \\
0.5 \\
0.95 \\
0.99 \\
\text { Mean }\end{array}$ & $\begin{array}{l}\text { Value } \\
0.000642227 \\
0.00150738 \\
0.0280373 \\
0.482445 \\
2.69899 \\
20.9685\end{array}$ & $\begin{array}{l}\text { CVM (o) } \\
1.55942 \\
1.19946 \\
1.03367 \\
1.45839 . \\
2.60364 \\
29.4023\end{array}$ & $\begin{array}{l}\text { Fractile } \\
0.01 \\
0.05 \\
0.5 \\
0.95 \\
0.99 \\
\text { Mean }\end{array}$ & $\begin{array}{l}\text { Value } \\
0.00223991 \\
0.006994 \\
0.135565 \\
4.11179 \\
34.18 \\
127.689\end{array}$ & $\begin{array}{l}\text { CVM }\left(\frac{8}{6}\right) \\
2.71725 \\
2.28426 \\
2.08139 \\
4.1132 \\
4.19975 \\
15.3807\end{array}$ \\
\hline Variance & $4.1733 \times 10^{6}$ & 56.4701 & Variance & $5.16397 \times 10^{7}$ & 25.8577 \\
\hline
\end{tabular}

Results from previous 2 analytic [U@.99] \& 1 targeted [U@.95,.99] methods (denominators) for V@.99, compared to corresponding results from 2 -D nested procedure:

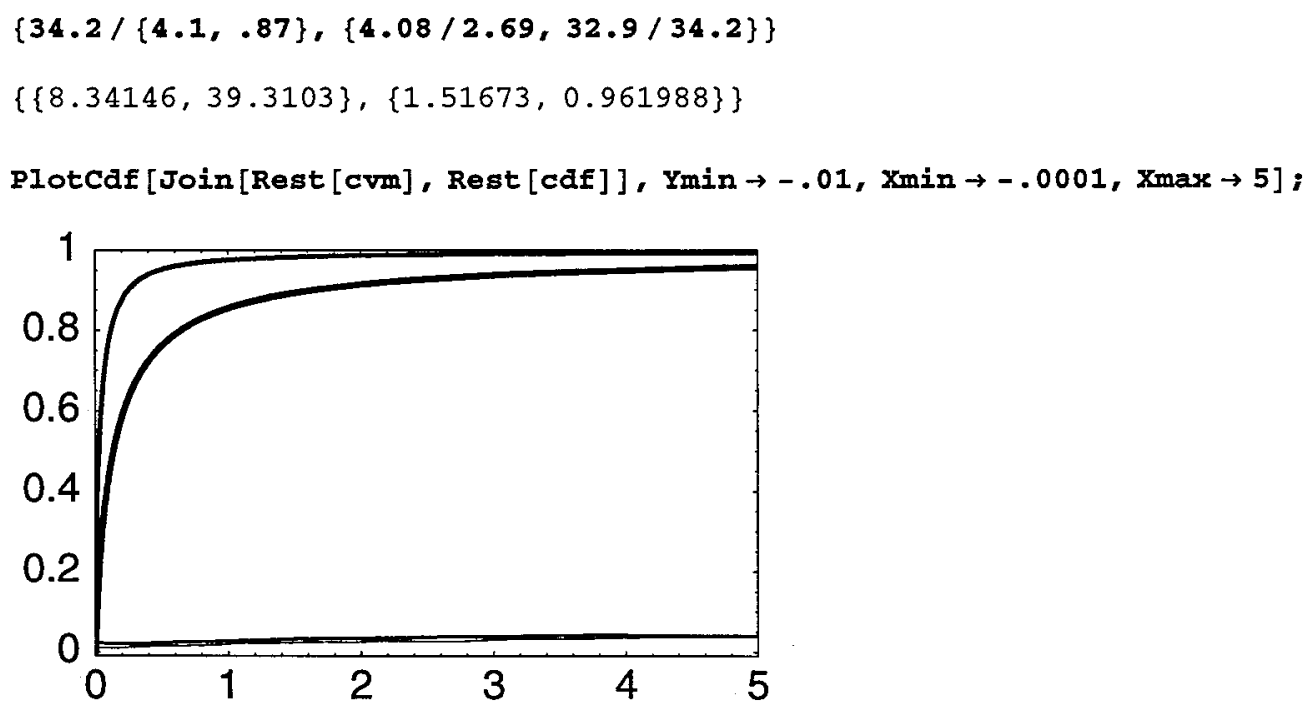


PlotCdf [Join [cvm, cdf], Ymin $\rightarrow-.01, X \min \rightarrow-.0001, X \max \rightarrow .25]$;

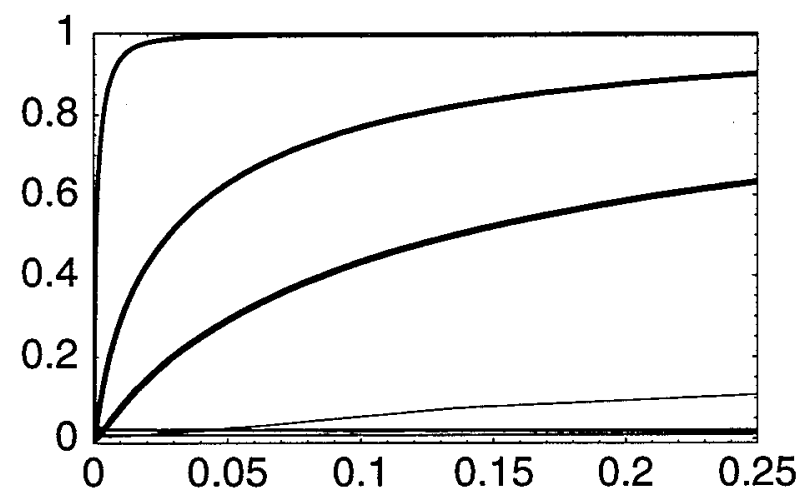

end

end

\section{Population Risk}

\# expected cases for different values of total-population size, $n$, via the relation: $\langle\mathrm{N}\rangle=\mathrm{n}(<\mathrm{Rbar}\rangle)$. Note that Rbar (and hence $<\mathrm{Rbar}>$ ) was scaled above by a factor of $10^{6}$, and so needs to be rescaled by $10^{-6}$.

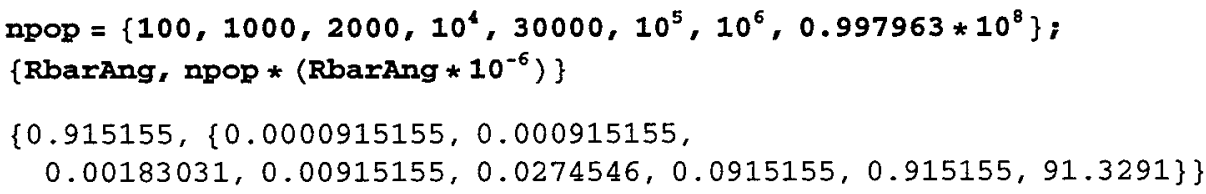

To obtain the likelihood $P_{0}$ of 0 people at risk associated with specified population sizes, first derive the probability mass function corresponding to Rbar, adjust to reflect the fact that its last element is artifically high due to how QUAnalyze defines this last element, and then use the adjusted pmf to calculate the complementary conditional Poisson likelihoods $\left\{P_{0}\right.$, $\left.1-P_{0}\right\}$ corresponding to specified population sizes (npop):

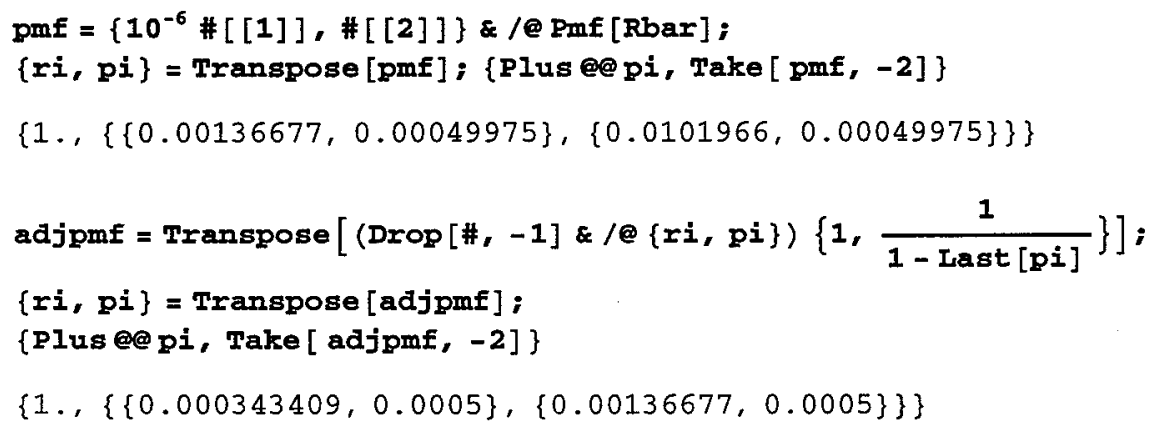




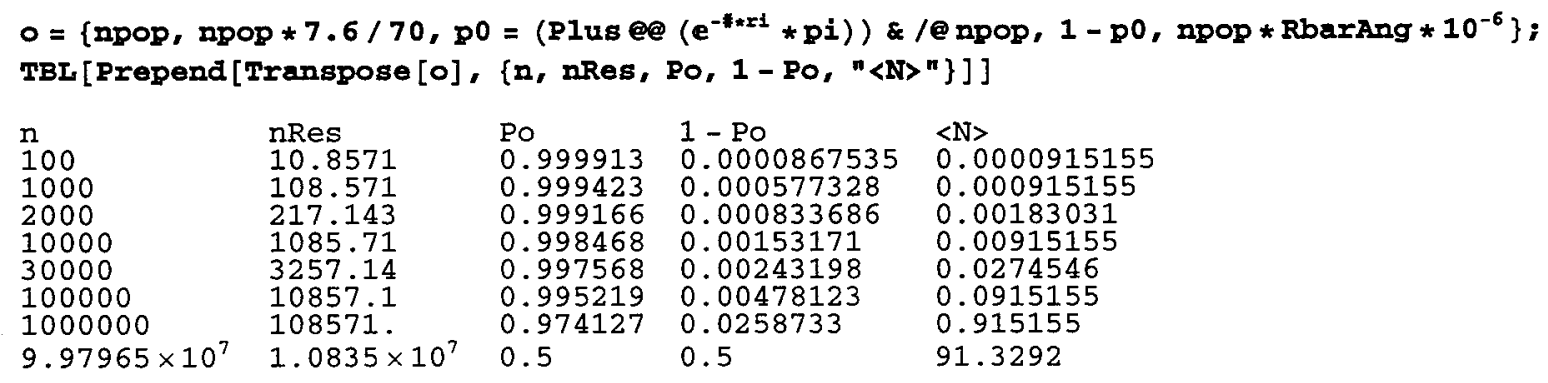

To obtain the \# people at risk associated with $P_{0}=0.5$, use the adjusted pmf to calculate the conditional Poisson likelihood corresponding to a likelihood of 0.50 (by manual numerical optimization [not shown]) :

Plus $\left(e^{-0.997963 * 10^{8} * x i} * p i\right)$

0.5

Thus, only if about $\mathbf{1 0 0}$ million people were exposed would it be more likely than not that there would be 1 or more cases.

end 


\section{Appendix 3.I \\ Functions Used}

\section{- Mathematica ${ }^{\circledR}$ functions}

Note: The following Mathematica shorthand notation was used that is not included in the list of functions below:

$a+b=a$ plus $b$

$a-b=a$ minus $b$

$a b=a * b=$ the product of $a$ and $b$

$a / b=a$ divided by $b$

$a^{\wedge} b=a$ to the power of $b$

$\{a, b, c, \ldots\}=$. List $[a, b, c, \ldots]=a$ "list" (i.e., array, vector, or set) of elements $a, b, c, \ldots$.

$\mathrm{fxn} / @\{a, b, c, .\}=.\operatorname{Map}[\mathrm{fxn},\{\mathrm{a}, \mathrm{b}, \mathrm{c}, \ldots\}]=.\mathrm{a}$ new list made by mapping (i.e., applying) the function fxn onto each member of the list $\{a, b, c, \ldots\}$

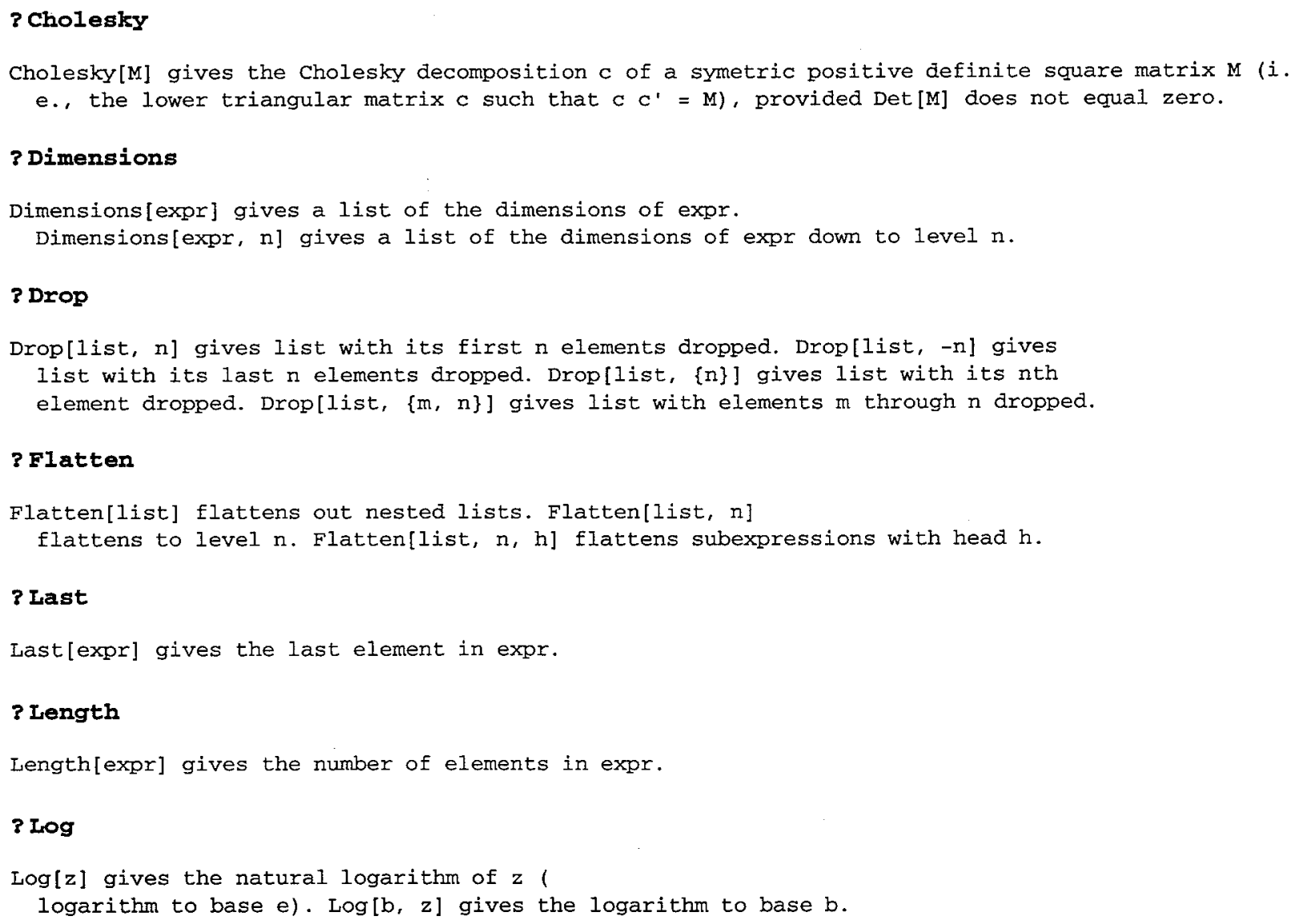




\section{MapThread}

MapThread $[f,\{\{a 1, a 2, \ldots\},\{b 1, b 2, \ldots\}, \ldots\}]$

gives $\{f[a 1, b 1, \ldots], f[a 2, b 2, \ldots\}, \ldots\}$. MapThread $[f,\{\operatorname{expr} 1$,

expr2, ... $\}, n]$ applies $f$ to the parts of the expri at level $n$.

\section{? Max}

$\operatorname{Max}[x 1, x 2, \ldots]$ yields the numerically largest of the $x i . \operatorname{Max}[\{x]$, $x 2, \ldots\},\{y 1, \ldots\}, \ldots\}$ yields the largest element of any of the 1 ists.

\section{? Min}

$\operatorname{Min}[x 1, x 2, \ldots]$ yields the numerically smallest of the $x i$. Min $[\{x 1$, $x 2, \ldots\},\{y 1, \ldots\}, \ldots]$ yields the smallest element of any of the lists.

\section{? Prepend}

Prepend[expr, elem] gives expr with elem prepended.

\section{? Range}

Range [imax] generates the 1 ist $\{1,2, \ldots$, imax $\}$ Range [imin, imax] generates the list \{imin, ..., imax\}. Range[imin, imax, di] uses step di.

\section{? Solve}

Solve[eqns, vars] attempts to solve an equation or set of equations for the variables vars. Solve[ eqns, vars, elims] attempts to solve the equations for vars, eliminating the variables elims.

\section{? Sort}

Sort[list] sorts the elements of list into canonical order. Sort[list, p] sorts using the ordering function p.

\section{? Take}

Take[list, $n$ ] gives the first $n$ elements of list. Take[1ist, $-n]$ gives the last $\mathrm{n}$ elements of list. Take[list, $\{\mathrm{m}, \mathrm{n}\}$ ] gives elements $\mathrm{m}$ through $\mathrm{n}$ of list.

\section{Transpose}

Transpose[list] transposes the first two levels in

list. Transpose[list, $\{n 1, n 2, \ldots\}]$ transposes list so that the levels $1,2, \ldots$ in 1 ist correspond to levels $n 1, n 2, \ldots$ in the result.

\section{RiskQ 4.0 functions}

$<$ RiskQ;

\section{? Averagecde}

Averagecdf[cdfs, options:] generates a cdf which is the exact average of the input list of cdfs and/or cmfs. By default, the input cdfs are equally weighted ( i.e., all cdfs are assumed to be equally likely). Use Weights $\rightarrow$ weights to specify weights. Use TestCaf $\rightarrow$ False to suppress automatic CafQ test of input cafs. Use Approximate $\rightarrow \mathrm{n}$ (or $\rightarrow \mathrm{xlist}$ ) to return an approximation of the true average cdf evaluated at $n>1$ equal abcissa intervals (or at the supplied list of abcissa values). 
? Caf

Cdf[x, options] returns a matrix representing a cdf (cumulative distribution funtion) from which $x$ is (assumed to be) sampled if $x$ is a vector. The first point is $\{x 10,0\}$ where $x l o$ is assumed to be $\operatorname{Min}[0, \operatorname{Min}[x]]$ unless $x l o<\operatorname{Min}[x]$ is entered with $X \min \rightarrow x l o$. The last point is $\{x h i, 1\}$ where $x h i$ is assumed to be Max $[x]$ unless xhi $2 \operatorname{Max}[x]$ is entered with $X \max \rightarrow$ xhi. Cdf estimates the cdf corresponding to $n$ samples of a continous random variate, using linear interpolation. Use Weights $\rightarrow$ w to obtain a cdf based on weight-vector $w$ corresponding to list (in which case the $\mathrm{xmax}$ option is ignored). If $\mathrm{x}$ is a cmf or a pmf, a corresponding cdf is output. Use Pmf $\rightarrow$ True (or the alternative function Pmf) to obtain the probability mass function (pmf) corresponding to 1ist. Use Simplify $\rightarrow$ False to suppress default distribution-simplification algorithm. To obtain the sample cdf (a step function) corresponding to list, or to model a discrete random variate, see Cmf. See also RQ.

\section{? Data}

Data[datarows, expr,$\ldots$ ] returns a list of data rows specified symbolically as a function of the input datarows list, where each datarows $s_{i}=\left\{x_{i 1}, x_{i 2}, \ldots, x_{i n}\right\}$ has $n$ columns, and expr $_{k}$ are Data arguments. If datarows is a list but not a list of lists, then it is assumed to specify a single data column. By default, datarows 1 must be a list whose $j$ th element (name $j^{\text {) }}$ is a unique symbol or string used to name the variate whose values $\mathrm{x}_{i j}$ appear in the rest of the $j$ th data column for $j=1, \ldots, n$; however, if $\operatorname{expr}_{1}$ is a vector containing $n$ symbols and/or strings, then expr $r_{1}$ name $_{j}$ is assumed. If $\operatorname{expr}_{1}$ is a non-Rule expression (e.g., involving any of the name ${ }_{j}$ ), then expr $r_{1}$ is returned evaluated using the specified data column(s). Otherwise, expr $r_{k}$ must specify one or more of the following options (described below) to transform datarows: Append (or Replace), Classify (or Bin), Complement, Drop, Fill, Interpolate, Intersection, Merge, Names, Number, Rename, Restructure, Set, Shift, SortBy, Take, and/or Union. These

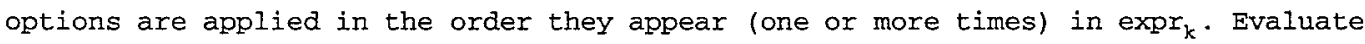
Data[option] to get information about any Data option. Column names (e.g., name $)_{1}$ appearing in any of these options are assumed to be among those defined for (e.g. as the 1st row of) datarows; any corresponding reassigned name (e.g., $X$ after the assignment $X=n^{2} e_{1}$ has been made) used should appear as an argument of HoldForm (e.g., as HoldForm[X]). Data should be nested only if the nested expr is a rule or rule sequence.

\section{? EV}

EV [ $x$, options] returns the arithmetic average of (e.g., a vector) $x$, or the expected value of $\mathrm{x}$ if $\mathrm{x}$ is a valid cdf, cmf or pmf. If $\mathrm{x}$ is a vector, Weights $\rightarrow \mathrm{w}$ may be used to obtain the weighted average value corresponding to the weights-vector $w$ applied to $x$. If $x$ is a cdf with $>2$ evenly spaced ordinate values (i.e., evaluated at equal probability intervals) and Empirical $\rightarrow$ True, then the minimum and maximum abscissa values are ignored.

\section{? Edf}

Edf: See Evaluatecdf, RQ.

\section{? Evaluatecde}

Evaluatecdf[cdf, x, complement:False] calculates the probability $p$ that a random variate distribituted as cdf is less than or equal to $x$, using linear interpolation. If a third argument, True, is included, the output probability is 1$p$. The input $x$ may be a list, in which case a corresponding output list is generated. 
P FIT

FIT[xy, fxn_List, $x \_S y m b o l$, options] fits the General Linear Model (GLM), y(x) = Sum[qi $\left.F i(x)\right]$ for $i=1 \ldots n p$, to $x j-y j$ data (for $j=1 \ldots n$ ) given in $x y$ (an $n$-by-2 matrix) by direct or generalized least-squares regression, assuming yj are normally distributed as $N(E y j$, Sqrt [v/wj]) with $w j=1$ by default and $v$ estimated by the mean square of $y$-residuals ( unless KnownVariances $\rightarrow$ True is used, in which case $y j \sim N\left(0, S q r t\left[1 / w^{j}\right]\right.$ is assumed) . Use NYat $\mathrm{X} \rightarrow \mathrm{nYj}$, with integer nyj>0 (nY=1 by default) or nyj an $n$-lengthed such 1 ist, to treat $y j$ as means, in which case corresponding sample stand. devs. sj of nyj $y-$ values must be specified using Errors $\rightarrow$ sj. Use Weights $\rightarrow$ wj to similarly specify known weights $w j$; or use Weights $\rightarrow\{$ Wyhat, yhat, df $\}$ to specify that $w j=($ Wyhat $\mid$ yhat $=y(x j))$ or that $w j=($ Wyhatj|yhat $=y(x j))$--where wyat is an expression (or Wyhatj is a list of $\mathrm{n}$ expressions each) involving the symbol yhat--in which case the fit is obtained by iterative reweighting assuming df $(=0$ if not specified) extra degrees of freedom are lost in estimating What from the data (\& use MaxIterations $\rightarrow$ maxit and/or Tolerance $\rightarrow$ tol to override defaults). If Report $\rightarrow$ True, SDs and $100 \mathrm{p} \%$ conf. limits on ai, $\mathrm{R}^{2}$, a chi-square test-of-fit, ANOVA table, F-tests of GLM-fit and nonzero qi for $i>0$, and a plot are all printed (use Report $\rightarrow$ Plot to add a plot). The qi estimates are output, along with: covariance matrix, the list \{xval, yhat,yLCL, yUCL\}, a sum-of-squares \& assoc. degree-offreedom matrix, the F-values and their p-values, the chi-square value and its $p$-value, the fitted function, and/or a plot using output $\rightarrow\{\mathrm{CV}, \mathrm{xva1}$, SumSquares, F, PvalF, X2, PvalX2, BestFit, [and/or] Plot\}, where xval may be a list. Use Confidence $\rightarrow p$ to change p from 0.95, and use Xmin $\rightarrow x 10$, etc. (see PlotData options) to change plot defaults.

\section{? Idf}

Idf: See Inversecdf, RQ.

\section{Inversecdf}

Inversecdf[cdf, p, options] evaluates cdf at the cumulative probability value $p$, for any valid cdf or $\mathrm{cmf}$. The input $\mathrm{p}$ may be a list of probability values, in which case a corresponding output 1 ist is generated. Use TestCdf->False to suppress default CdfQ test.

\section{? Normalcdf}

Normalcdf $[\mathrm{z}, \mathrm{s}:, \mathrm{n}: 100]$ = the standard Gaussian cdf; i.e. the probability $\mathrm{p}$ that $\mathrm{z}<=\mathbf{z}$ for real $\mathrm{z}$ and standard normal random variate $\mathrm{z}$. If $\mathrm{z}$ is a list, $\mathrm{p}$ is the corresponding list. If $s$ is set to Inv, then the inverse standard Gaussian caf is returned for argument( $s$ ) $\mathbf{z}$ where $0<=\mathbf{z}<=1$. If $\mathbf{s}$ is entered as a nonegative real number, then an approximate cdf is returned corresponding to the parameters $\{z=$ mean, $s=s t a n d$. dev. $\}$ for a nonstandard random variate $\mathrm{z}$, evaluated at $\mathrm{n}$ equiprobable quantile intervals. In evaluating the inv. stand. Gaussian cdf for Min[p,1-p]>2.21 10^-7, Normalcdf makes use of an 11th-order polynomial approximation with an absolute error $<0.50310^{\wedge}-6$.

\section{P Plotcdf}

Plotcdf[cdfs] returns a plot of a cdf or of several cafs entered as a list of cdfs. See Plotoptions.

\section{P PlotData}

PlotData[data, options:] plots an N-by-2 (or ( $x$ vs $y$ ) data set (DS), or a list of $n$ such sets, with points joined by lines (unless JoinPoints->False is used). Change point style with Style->list which by defualt is $\{O O, O A, O B, O V, O D, O, A, B, V, D\}=$ \{open Point, Triangle, Square, InvTriangle, Diamond,...(\& their solid equivs.)\}; use \{TO,TA,TB,TV,TD\} for transparent open symbols; use $\{P, X, M, I\}$ for \{plus,cross, dash,bar\}; \& use J to join points from adjacent DSs. Size and JoinPoints may be n-lengthed lists, where n<=Length [ Style] $<=2 n-1$ depending on how many Js are in Style; JoinPoints->False is enforced for DSs referenced by $J$ in Style.Use FitTo- $\{f[x], x\}$ to include a plot of $f[x]$ (which may be a list of functions) vs. $x$. Use data=Plot to plot functions only. See Plotoptions. 


\section{? QUAnalyze}

QUAnalyze[cdfs, Fxn, nsam, nsim, options:] performs a quantitative uncertainty analysis involving simulated values $F_{x n}$ of $F x n\left[\operatorname{var}_{1}, \ldots, v r_{n}\right]$, where $F x n$ is a user-defined listable function, $j=1, \ldots$ nsam, and $k=1, \ldots$ nsim. Uncertainties in $\operatorname{var}_{i}, i=1, \ldots, n$, are specified by the corresponding input cumulative probability distributions, cdfs = $\left\{\operatorname{cdf}_{1}, \ldots, \mathrm{cdf}_{n}\right\}$, where each $\mathrm{cdf}_{i}$ must be either a valid cdf object (for which TrueQ $\left.\mathrm{cdf}_{i}\right]==$ True) or a valid symbolic cdf-specification (see Simulatecdf). All cdfs are by default uncorrelated, unless correlate $\rightarrow T$ is used to specify $T$ as the target rankcorrelation matrix (or as its upper-right rows--see Reflect). The list (SimReport, cdfFxn, CvmFxn is output, where: SimReport lists the coefficients of variation (as a 8 ) of $\operatorname{EV}\left[\mathrm{FXn}_{j}\right]_{k}$ and corresponding p-fractiles of Fxn, the maximum of Jennrich chi-square values assessing homogeneity with $T$, its degrees of freedom, and the corrsponding Hommeladjusted p-value; cdfFxn characterizes Fxn uncertainty (as the means of nsim sorted sets of nsam sample values of Fxn--i.e., as nsam mean Fxn-fractile values--where nsam > $\mathrm{n}$ and nsam>nsim>1); and cvmFxn lists the corresponding coefficients of variation of the nsam fractile means (and so summarizes corresponding Monte-Carlo sampling error). By default, the minimum and maximum possible values of Fxn are assumed to be $x l o=$ Min [ $\left.\mathrm{Fxn}_{j k}\right]$ and $\mathrm{xhi}=\operatorname{Max}\left[\mathrm{Fxn}_{j k}\right]$, respectively; use $\mathrm{Xmin \rightarrow xmin}$ and/or $\mathrm{Xmax} \rightarrow \mathrm{xmax}$ to change these defaults (provided $x$ min $<x 10$ and $x$ max $>x h i$ ). Use Fractiles $\rightarrow p$ to specify the list of p-fractiles of $\mathrm{Fxn}$ to be used to summarize simulation quality. Use SimIn $\rightarrow \mathrm{ML}$ to specify the cdf-simulation values to be used, where either: (1) ML is a list of nsim matrices each n-by-nsam in dimension (as output by simulatecdf); or (2) ML is a list of nsim elements each of the form $\{M$, RankCorrelations $\rightarrow R$, Jennrich $\rightarrow\{x 2, d f, p\}\}$ (i.e., each element of $\mathrm{ML}$ is a list of the form output by Simulatecdf using the Report $\rightarrow$ Append option), where $M$ is an n-by-nsam matrix, $R$ is an $n$-by-n matrix, and $x 2$, $d f$, and $p$ are numbers with $0<p<1$ ). If SimIn is specified, the QUAnalyze cdfs argument supplied may be the integer $\mathrm{n}$. Note that cdf and cvm may be plotted together because they use common abscissa values, which are scaled by an n-fold factor if the option scale $\rightarrow \mathrm{n}$ is used.

\section{? Reflect}

Reflect[upper, diagvec:Automatic, anti:False] returns an $n-b y-n$ symetric square matrix $M$ given upper, an $(n-1)$-length list of $(n-1-i)$-length lists $(i=1, \ldots, n)$ that represent the first $n-1$ rows of upper elements (without the diagonal elements) of M. The diagonal is a vector of ones by default, or may be entered as the 2 nd argument (either a constant or an n-length list). If the $3 \mathrm{rd}$ argument is set to True, then the corresponding antisymetric matrix is returned.

\section{? RQ}

$\mathrm{RQ}$ [operation, distribution, parameter(s), z] performs an operation Cdf (=cumulative distribution function), $\mathrm{E}=\mathrm{M}=\mathrm{Mean}$ (=expected value), V=Var=Variance, $\mathrm{D}=$ Range=Domain, $\mathrm{P}=\mathrm{Pr}=\mathrm{Prob}=\mathrm{PDF}, \mathrm{C}=\mathrm{Edf}=\mathrm{CDF} \quad(\mathrm{CC}=\mathrm{C}$ complement), $\mathrm{Q}=\mathrm{Idf}=\mathrm{Quantile}$ ( $\mathrm{QC}=\mathrm{Q}$ complement), or Test= (test validity of 2 nd \& $3 \mathrm{rd} R Q$ parameters) on a $\mathrm{B}=\mathrm{Beta}, \mathrm{B} i=\mathrm{Binomial,} \mathrm{X} 2=$ ChiSquare, E=Exponential, F=FRatio, G=Gamma, Geo=Geometric, H=Hypergeometric, LN= LogNormal, Lg=Logistic, NBi=NegativeBinomial, N=Normal, Psn=Poisson (=P), T=StudentT, Tri=Triangular, U=Uniform, W=Weibull (=Wb1), or M=Empirical (=Cdf=Cmf) distribution with the specified parameter value(s) or for the particular cdf/cmf, at the point(s) $z$. If $z$ is included with the cdf operation, the output cdf is given for $z+1$ points.

\section{? Simplifycdf}

SimplifyCdf[cdf] returns any valid input cdf or cmf in its simplest possible form, that is, without any unnecessarily repeated or redundant elements. 


\section{? Simulatecdf}

Simulatecdf[cdf(s), nsim, options:] generates a list of nsim values simulated from an input cdf, or of $n$ lists of nsim values with the ith list simulated from the $i$ th of an input list of $\mathrm{n}$ cdf objects with a target rank- correlation matrix $\mathrm{T}$. Input $\mathrm{T}$ using Correlate $\rightarrow T$ for a square matrix $T$ (or its upper-right rows--see Reflect); by default $T$ is an identity matrix. Each cdf must be either empirical (such that TrueQ[cdf]== True) or a valid \{type, par\} cdf-specification (see RQ). Simulations use a Systematic Latin Hypercube (SLH) method, adjusted (unless Correlate $\rightarrow$ False is used) to yield variates whose true rank-correlation matrix $R$ approximates $T$. Alternative methods may be specified with simMethod $\rightarrow$ LatinHypercube $(\rightarrow \mathrm{LH})$ or $\rightarrow$ Random $(\rightarrow U, \rightarrow$ Uniform). If the first argument is entered as $n$ for $n>0$, then $\mathrm{cdf}(\mathrm{s})$ are assumed to be $\mathrm{n}$ standard normal cdfs and $T=R$ is the actual product-moment correlation matrix. Note that unless Correlate $\rightarrow$ False, nsim must be $>n$. Use Report $\rightarrow$ False to suppress the Jennrich- function report comparing T VS.R (suppressed by default for normal variates, for which $T=R$ ), or use Report $\rightarrow$ Append to append $R$ and $\{\operatorname{chi} 2, d f$, pval\} from this report to the output (see Jennrich). Use SimIn $\rightarrow$ mymatrix (Simout $\rightarrow$ True) to use an input (output the simulated) rank-matrix. Use Testcdf $\rightarrow$ False to save time if $\mathrm{Cdf} Q[\mathrm{cdf}]==$ True for each input cdf.

\section{? Standardizecaf}

Standardizecdf[incdf, Values, options:] returns a new cdf based on linear interpolation of incof (any cdf or cmf) evaluated at Values, where values are assumed to be probability values, except that values are treated as cdf abcissa values if any of the Values are $<0$ or $>1$ or if the option Probabilityvalues $\rightarrow$ False is used. Values must be either a list or an integer $>0$; in the latter case cdf-evaluation occurs in $n$ equal increments over the specified range of probability or abcissa values. If the Midpoints $\rightarrow$ True option is used, then cdf-evaluation occurs at the midpoints of the successive element-pairs in the specified set of values, rather than at those values themselves. If incdf is a list of cdfs, then a corresponding list of standardized cdfs is output. Use TestCdf $\rightarrow$ False to suppress automatic CdfQ test of input cdfs.

\section{? TBL}

TBL $[x]=$ TableForm $[x$, TableSpacing->1]. TBL $[x, n]=$ TableForm [ $x$, TableSpacing- $>n]$. TBL $[x, n, r]=$ TableForm [Take $[x, r]$, TableSpacing- $>n]$.

\section{? WriteMatrix}

WriteMatrix[filenamestring, dataMatrix, separatorstring] writes a data matrix to the specified filename as an ASCII file. The separatorstring is a tab by default. 


\title{
n Other Functions
}

\author{
? IsMin \\ LSMin $[x, y, p, f x p, o p t i o n s]$ Attempts to reduce $x 2$, i.e., the chi-square (i.e., weighted sum of \\ squared residuals) between $a$ list $y$ of data and a user-defined function $\operatorname{fxp}[x, P]$ of \\ corresponding independent-values $x$ and parameters $P$, starting with the initially guessed \\ parameter list $p$, returning \{phat, sd, $\{x 2, d f, p\}\}$ where phat is the 1 ist of asymptotic \\ maximum-likelihood parameter-value estimates, sd is the corresponding list of standard \\ deviations (or the full covariance matrix if output->CVM is used), $x 2$ is the goodness- \\ of-fit chi-square value, $d f=$ (Length[y] - \# est. parameters), and $p$ is the corresponding \\ p-value. Use NYatX->ny if the $y$-values are the means of (a list of) ny corresponding \\ values, with corresponding standard deviations sdy all 1 (unless SDY->sY is used). It \\ is assumed that $y j \sim \mathrm{N}\left(E y j\right.$, Sqrt $\left[\mathrm{v} / \mathrm{wj}^{\mathrm{j}}\right]$ ) with $w j=1$ by default (and $\mathrm{v}=$ mean square of $\mathrm{y}$ - \\ residuals if KnownVariances- $>$ False is used, in which case $p$ is meaningless; otherwise \\ $\mathrm{v}=1$ ). Use Weights->wj to specify weights $w j$. Use weights->\{Wyhat, yhat, df $\}$ to specify \\ $\mathrm{w} j=$ (Wyhat $\mid$ yhat=y $(\mathrm{x} j)$ ) for Wyhat an expression (or list of $\mathrm{n}$ expressions each) involving \\ the symbol yhat, in which cases the fit is obtained by iterative reweighting. If weights \\ are not specified, KnownVariances-> False is assumed. Use Parameters-> pinlist with \\ ordered integer index-list pinlist to restrict optimization to a subset of $p$ specified by \\ pinlist. The search stops if reductions in chi-square become less than tol=0.001 (reset \\ using Tolerance->tol) or if iterations > maxit=100 (reset using Maxit->maxit; output \\ appended with 'Warning'). Set Progress->True to see intermediate output (at precision $p$ \\ using SeePrecision-> p). Levenberg-Marquardt minimization of the chi-square objective \\ function is used (WH Press et al., Numerical Recipies, Cambridge U. Press, New York, \\ 1986, pp. 521-528), with shifts at each step having a relative size equal to 1000 \\ (reset using step->size). In the case of unknown sigy, generalized (i.e., iteratively \\ reweighted) $\times 2$-minimization is performed (see Carrol and Rupert, Transformation \\ and Weighting in Regression, Chapman and Hall, New York, 1988). Needs MarqCof, \\ Partial, Bracket, ParaMin, and Mathematica's CDF and ChiSquareDistribution functions.
}

\section{? MSDx}

MSDX[GMx, GSDx] returns the arithmetic mean and arithmetic sdandard deviation of a lognormal variate $\mathrm{X}$ that also has the specified geometric mean GMx and geometric sdandard deviation GSDx, based on the method of moments.

\section{? GMGSDX}

GMGSDx[Mx, SDX] returns the geometric mean and geometric sdandard deviation of a lognormal variate $\mathrm{X}$ that also has the specified arithmetic mean $\mathrm{Mx}$ and arithmetic sdandard deviation SDx, based on the method of moments.

\section{? GMGSDX1}

GMGSDX1[cvWant, cv2] returns the GM and GSD of a lognormal variate $\mathrm{X} 1$, such that the product $\mathrm{X} 1 * \mathrm{X} 2$ has the desired coefficient of variation ( $\mathrm{CV})=\mathrm{cvWant}$, conditional on the lognormal variate $\mathrm{X} 2$ having an arithmetic mean and $\mathrm{CV}$ equal to 1 and $\mathrm{CV} 2$, respectively, based on the method of moments. 